\title{
Topics in Portfolio Choice: Qualitative Properties, Time Consistency and Investment under Model Uncertainty
}

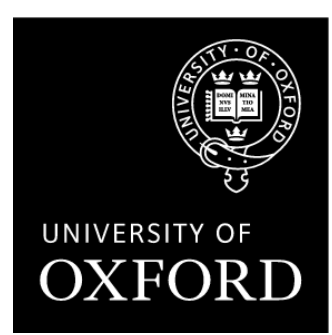

Sigrid Källblad Lady Margaret Hall University of Oxford

A thesis submitted for the degree of

Doctor of Philosophy

Trinity 2013 
Till Ingegerd, Ivar och Ulrik 


\section{Acknowledgements}

Foremost, I would like to thank my supervisors Jan Obłój and Thaleia Zariphopoulou without whom this thesis would not have been possible. I thank them for their guidance, for helpful and stimulating discussions throughout this time and for their constant support.

My thanks also go to the faculty and my fellow students within the Mathematical and Computational Finance Group in Oxford and at Oxford Man Institute for creating such a nice and stimulating working environment. I also gratefully acknowledge the financial support from Dr Marcus Wallenberg's foundation, Lady Margaret Hall together with Santander Bank, Oxford Man Institute and Svenska försäkringsföreningen.

I would further like to thank Alex Schied and Xun Yu Zhou for valuable comments on the contents of this thesis. I also thank Tomas Björk for his support and guidance over the years. A special thanks goes to Mia Hinnerich and Jana Madjarova. I also thank Michalis Anthropelos, Christer Borell, Sam Cohen, Kjell Holmåker, Hanqing Jin, Michael Kupper, Mike Monoyios, Sergey Nadtochiy, Johannes Ruf and Gordan Žitković for helpful discussions at various points. Judith Campos, María Suárez Taboada and Sofia Piltz, thanks for friendship and fruitful discussions. A special thanks goes to Martin Larsson for enjoyable discussions over the years.

My heartfelt thanks go to Karolina Bujok, Franziska Jahnke and Serena Lunardi for making our stay in Oxford such a memorable time; to Jossan, Anne, Helen and Lisa, for staying close despite the distance; and last, but not least, to my family. 


\begin{abstract}
The study of expected utility maximization in continuous-time stochastic market models dates back to the seminal work of Merton 1969 and has since been central to the area of Mathematical Finance. The associated stochastic optimization problems have been extensively studied. The problem formulation relies on two strong underlying assumptions: the ability to specify the underpinning market model and the knowledge of the investor's risk preferences. However, neither of these inputs is easily available, if at all. Resulting issues have attracted continuous attention and prompted very active and diverse lines of research. This thesis seeks to contribute towards this literature and questions related to both of the above issues are studied. Specifically, we study the implications of certain qualitative properties of the utility function; we introduce, and study various aspects of, the notion of robust forward investment criteria; and we study the investment problem associated with risk- and ambiguity-averse preference criteria defined in terms of quasiconcave utility functionals.
\end{abstract}




\section{Contents}

1 Introduction 1

2 New insights for optimal portfolios and risk preferences when investing in log-normal markets $\quad 12$

2.1 Qualitative analysis of optimal investment policies and robustness of risk tolerance 12

2.1.1 The log-normal investment model . . . . . . . . . . . . . . . . 15

2.1.2 Auxiliary equations, harmonic functions and feedback controls . . . . . . 18

2.1.2.1 The optimal portfolio and wealth processes . . . . . . . . 22

2.1.3 Spatial and temporal properties of optimal allocations and optimal weights 23

2.1.3.1 Monotonicity in wealth . . . . . . . . . . . . . . . 24

2.1.3.2 Concavity/convexity in wealth . . . . . . . . . . 25

2.1.3.3 Monotonicity in time . . . . . . . . . . . . . . . 27

2.1.4 Robustness of the optimal allocations and optimal weights . . . . . . . . 28

2.1.5 Sensitivity analysis . . . . . . . . . . . . . . . . . . 35

2.1.5.1 Sensitivities with respect to market parameters . . . . . . . 35

2.1.5.2 Sensitivities of the optimal processes in terms of the initial wealth 38

2.2 Structural representations of utilities and their effects on horizon flexibility and stochastic dominance . . . . . . . . . . . . . . . . . . . . . . . . . 39

2.2.1 Complete monotonicity and two specific classes of utility functions . . . . 40

2.2.2 Preservation of stochastic dominance - complete monotonicity of the marginals 42

2.2.3 Horizon flexibility - complete monotonicity of the inverse marginals . . . . 44

2.2.4 The role of complete monotonicity in portfolio choice . . . . . . . . . . 48

2.2.5 Investment policies and the inverse investment problem . . . . . . . . . 49

2.2.6 Auxiliary results on completely monotonic functions . . . . . . . . . . 51

3 Optimal investment and consumption for forward stochastic utilities $\quad 58$

3.1 Connection of forward performance criteria with the classical utility maximization 58

3.1.1 Definition and general remarks . . . . . . . . . . . . . . . . 59 
3.1.2 Propagation of initial preferences and multiplicity of criteria . . . . . . 60

3.1.2.1 Stochastic utility functions and the specification of initial prefer-

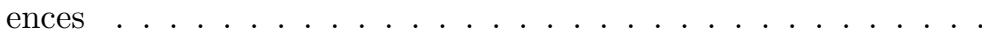

3.1.3 Volatility of the forward process and its connection with the classical formulation ........................ 64

3.1.4 Additional requirements on the forward criteria . . . . . . . . . . . 69

3.1.4.1 Deterministic functions of stochastic factors . . . . . . . . . . 69

3.1.4.2 Time monotone (non-volatile) criteria . . . . . . . . . . . 70

3.2 Forward investment and consumption criteria . . . . . . . . . . . . . 72

3.2.1 Definition of forward investment-consumption criteria . . . . . . . . . 73

3.2.2 Forward investment consumption criteria within a Brownian filtration and the associated SPDE . . . . . . . . . . . . . 74

3.2.3 Time-monotone forward investment-consumption criteria . . . . . . . 76

3.2.3.1 The associated random PDE and its primal and dual versions . 77

3.2.3.2 Explicit characterization of the time-monotone criteria . . . . . 80

3.2.3.3 Optimal strategies, equivalent formulations and economic interpretation ...................... 85

4 Time-consistent investments under model uncertainty \& the robust forward $\begin{array}{lr}\text { performance criteria } & 90\end{array}$

4.1 Introduction . . . . . . . . . . . . . . . . . . . . . 90

4.2 The market model and definition of the criterion . . . . . . . . . . . . . . 91

4.2 .1 The market model and notation . . . . . . . . . . . . . . . . . 91

4.2.2 Robust forward performance criteria . . . . . . . . . . . . . . . 92

4.3 Dual characterizations of robust performance criteria . . . . . . . . . . . . . . 95

4.3.1 Further results on robust self-generation . . . . . . . . . . . . . . 98

4.3.1.1 Time-consistency of the value-field . . . . . . . . . . . . . 98

4.3.1.2 Submartingale property of the dual field . . . . . . . . . . . . . 99

4.3.2 Penalty functions associated with risk measures . . . . . . . . . . . . 100

4.4 Non-volatile robust criteria in a Brownian filtration . . . . . . . . . . . . . . . . 103

4.4.1 The Brownian setup . . . . . . . . . . . . . . . 103

4.4.2 Pinning down of robust forward criteria from the initial condition . . . 106

4.4.3 Fields with volatility in function of stochastic factors . . . . . . . . . 107

4.4 .4 Non-volatile criteria . . . . . . . . . . . . . . . . . . 107

4.5 Non-volatile logarithmic criteria — an illustrative example . . . . . . . . . . . . . 109

4.5.1 Logarithmic example - main result . . . . . . . . . . . . . . . . . 110

4.5.2 Fractional Kelly strategies - optimality and interpretation . . . . . . . . 112 
4.5.3 Existence of equivalent non-robust forward criteria . . . . . . . . . . 113

4.5.4 Further remarks on the volatility of the equivalent forward criteria . . . . 115

4.6 Proofs . . . . . . . . . . . . . . . . . . . . . 116

4.6 .1 Proof of Theorem $4.6 \ldots \ldots \ldots \ldots$

4.6 .2 Proof of Lemmas 4.11 and $4.14 \ldots \ldots \ldots \ldots$

5 Ambiguity averse portfolio optimization with respect to quasiconcave utility $\begin{array}{lr}\text { functionals } & 128\end{array}$

5.1 The market model and the investment criterion . . . . . . . . . . . . . . . 128

5.2 The main results . . . . . . . . . . . . . . . . . . . . . . 132

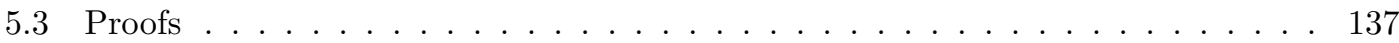

5.3.1 The auxiliary problem and its significance . . . . . . . . . . 138

5.3.2 Proof of the existence of an optimal investment strategy . . . . . . . . . 140

5.3 .3 Proof of the duality results . . . . . . . . . . . . . . 141

5.3.4 Proof of Lemma 5.12 and further remarks on the auxiliary problem . . . 146

6 Summary and Outlook $r 15$

Bibliography $\quad 155$ 


\section{Chapter 1}

\section{Introduction}

The optimal investment problem of choosing the best way to allocate an investor's capital is central to the area of mathematical finance. At its heart is the question of maximizing, over admissible trading strategies $\pi$, the expected utility of terminal wealth:

$$
\mathrm{E}\left[U\left(X_{T}^{\pi}\right)\right]
$$

From an economic point of view, the criteria arise from an axiomatization of risk-averse preferences by von Neumann and Morgenstern [118]. In a continuous-time stochastic market model, the problem dates back to the seminal contributions of Merton [91, 92]. Since then, it has remained one of the key topics within the area of mathematical finance. As such, the problem has a long and rich history and has given rise to an extensive volume of research. We limit ourselves to mentioning only the following landmarks: by use of convex-analytic duality methods, the problem was solved for general utility functions in complete Brownian models in Karatzas, Lehoczky and Shreve [72] and Cox and Huang [29]; duality techniques for incomplete models were first developed for the case of Itô-processes in Karatzas et al. [73], and for a general semi-martingale setting by Kramkov and Schachermayer [79, 80]. While these works provide existence of solutions under very general assumptions, stochastic control methods have been successfully used to obtain explicit results and characterise the solutions for various choices of utility functions and market settings. See, among others, Zariphopoulou [126] for a PDE-based approach to the choice of power utility within a stochastic factor model and Hu, Imkeller and Müller [67] for BSDE-based methods in a non-Markovian setting.

Despite the great deal of attention and numerous results obtained to date, several essential issues remain to be addressed. Indeed, the gap between the theoretical developments in portfolio optimisation and the methods used in practice often remains wide, and far more so than in other areas of mathematical finance such as derivative pricing and hedging. There are many practical 
features often overlooked on the theoretical side which may be co-responsible for this, such as: transaction costs, trading constraints and incomplete information, to mention a few. However, the fundamental reason seems to be that it is very difficult to provide the input required to compute the criterion in (1.1). Indeed, the investor needs to specify, on the one hand, her own preferences via the investment horizon $T$ and the utility function $U$ and, on the other, her views about the future and provide the probability measure to compute the expectation. Both issues turn out to be challenging and have attracted considerable attention, prompting a wide, diverse selection of works which attempt to address these issues.

This thesis seeks to contribute towards this literature and obtain a practically relevant approach to optimal portfolio allocation. Specifically, we study and relate a number of different approaches to utility maximization when there is uncertainty about the investment horizon, the utility function or the market model. At an abstract level, we are interested to see if we can weaken the assumptions on the investor's input and yet obtain meaningful results and insights on how to invest. By resting on previous developments, extending them and introducing new ones, we aim to demonstrate that this is indeed the case.

Next, the content of each chapter is presented and discussed.

\section{Chapter 2}

In Chapter 2, the traditional Merton problem of optimal investment in a log-normal market is studied. For this, by now, classical problem, new results are provided. Our work stems from the fact that, despite the extensive study of this problem and its extensions, very little is, yet, known about the qualitative behaviour of the optimal investment strategies, except when the utility is homothetic. We complement the literature by addressing these questions for more general utility functions.

In Section 2.1, we provide results on the spatial and temporal monotonicity and, furthermore, on the spatial concavity and convexity of the optimal portfolios. In order to relate these results to the literature, we recall that Arrow [5] showed, in a single-period model, that the optimal investment in the risky asset is increasing in wealth if, and only if, the investor's utility exhibits decreasing absolute risk aversion (DARA) and, furthermore, that the fraction of wealth invested, known as the average propensity to invest (API), is decreasing in wealth if, and only if, the utility exhibits increasing relative risk aversion (IRRA). Borell [19] was, to the best of our knowledge, the first to study such questions in a continuous-time setting. Specifically, he showed that, in a log-normal model, the monotonicity of both the absolute and relative terminal risk tolerance, are inherited by the so-called local risk-tolerance which is defined in a similar manner with respect to the value function. This, in turn, gives monotonicity properties of the optimal allocations and 
optimal weights. From a methodological point of view, we rely on the study of auxiliary partial differential equations. Hence, we obtain alternative proofs of these monotonicity results.

More importantly, our method allows for the study of further properties. Specifically, we show that in addition to the above monotonicity, the concavity and convexity, of the absolute risk tolerance are also inherited by the local risk-tolerance and, consequently, by the optimal allocation. This, in turn, yields results for the monotonicity, with respect to wealth, of the so-called marginal propensity to invest (MPI). Furthermore, these spatial properties are shown to be directly related to temporal properties of the optimal portfolio. While the study of the latter was not, naturally, an issue in single-period models, they have failed to receive appropriate attention even in discrete-settings. To our knowledge, Gollier and Zeckhauser [55] are the only ones who have studied it in a multi-period setting. They investigated the connection between horizon length/age of investors and curvature of their absolute risk aversion. Specifically, in [55] they prove that it is the convexity/concavity of the terminal risk tolerance which determines the time behaviour of the investment decisions. We extend this result to the continuous-time setting in that we show the following:

Result (Propositions 2.12 and 2.13). Let the absolute risk tolerance coefficient $R(x), x \geq 0$, (given in (2.11) below) be increasing and concave (convex) in wealth. Then, for each $t \in[0, T)$ and $x \geq 0$, the feedback function for the optimal allocations $\pi^{*}(x, t)$, is increasing and concave (convex) in wealth. Moreover, it is increasing (decreasing) in time.

This result is one of the main contributions of this chapter. It is in various ways surprising, for it shows that the popular wisdom that as investors get older they should decrease their allocations in the risky assets, holds only under certain assumptions on the terminal risk aversion.

In Section 2.1 we also address the question of whether optimal allocations are robust with respect to risk preferences. This is directly related to the question of whether two individuals with ordered risk aversion coefficients invest in an analogous way at all trading times. This has been analysed extensively in single- and multi-period settings. In continuous time, such a result was established in Xia [125] for log-normal stock prices. Here, we provide a far shorter and different in spirit proof of this result.

Result (Proposition 2.16). Let the absolute risk tolerance coefficients $R_{1}(x)$ and $R_{2}(x)$ satisfy, for $x \geq 0$, that $R^{\prime}(x)<\infty$ and $\left(R^{2}(x)\right)^{\prime \prime} \leq K$ for some $K \in \mathbb{R}$, and

$$
R_{1}(x) \leq R_{2}(x)
$$

Then, for $(x, t) \in \mathbb{R}_{+} \times[0, T)$, the optimal feedback allocations satisfy $\pi_{1}^{*}(x, t) \leq \pi_{2}^{*}(x, t)$. 
While the result holds for a slightly smaller class of solutions than that in [125], it bypasses several lengthy penalization and approximation arguments, and is based on entirely different PDE arguments.

In Section 2.2, further structural properties of the utility function and its effects on the portfolio optimization problem are examined. Specifically, we examine the role of complete monotonicity. First, the so-called completely monotone utilities are considered. They were introduced by Brocket and Golden [22] with the characteristic property that the marginal utility is a completely monotonic function. Second, we introduce a class of utility functions for which the inverse of the marginal is a completely monotonic function of a specific type. While the former utilities exhibit the property that the utility maximizer respects stochastic dominance of all orders, classical results by Widder [123] yield that the latter allow for the utility maximization problem to be extended to arbitrary horizons in a consistent way. Hence, the role of completely monotonic functions and the related trade-off between stochastic dominance and horizon flexibility is studied.

We note that the results in Chapter 2 provide information about the investment problem and its solution based on qualitative information about the utility function and not its exact specification. Hence, these results provide partial answers to our motivating question as to whether investment advice can be obtained also when the assumptions on the inputs, in this case the utility function, are relaxed.

\section{Chapter 3}

In Chapter 3, we study the notion of forward investment performance. It was introduced by Musiela and Zariphopoulou [94, 96, 97] as a complementary notion for the study of optimal portfolio choice; see also Henderson and Hobson [61,62] for an independent study of a closely related notion. Forward criteria (also referred to as progressive dynamic utilities and self-generating utility random fields) addresses the difficulties associated with the a priori specification of the investor's horizon and preferences. Recent studies includes the work by Anthropelos, Berrier, Rogers, El Karoui, Mrad, Tehranchi, Nadtochiy, Zariphopoulou and Žitković; see $[4,8,9,37,38,39,98,99,128,129]$.

A progressively measurable process $U: \Omega \times \mathbb{R}_{+} \times[0, \infty) \rightarrow \mathbb{R}$ is called a forward performance process if

i) the mapping $x \rightarrow U(x, t)$ is strictly concave and increasing,

ii) for each admissible investment strategy $\pi$, it holds that $\mathrm{E}\left[U_{t}\left(X_{t}^{\pi}\right) \mid \mathcal{F}_{s}\right] \leq U_{s}\left(X_{s}^{\pi}\right)$ for $s \leq t$,

iii) there exists an optimal $\pi^{*}$ for which equality holds in ii) for all $0 \leq s \leq t<\infty$. 
In other words, at each point in time, $U(\cdot, t)$ is a feasible utility function and the field is tied together via a condition of dynamic consistency. In the first part of this chapter, we provide a discussion of the notion and an overview of the results to date. This part does not contain any original material but the aim of the discussion, which is formal, is to provide intuition for the notion of forward performance. This is done via a comparison with (an inverse formulation of) the classical utility maximization problem and emphasis is put on connecting the results in previous and forthcoming chapters with the existing literature.

Specifically, as noted in [96] (cf. also [38]), within a Brownian framework, forward criteria constitute solutions to the following SPDE:

$$
d U(x, t)=\frac{1}{2} \frac{\left|\lambda_{t} U_{x}(x, t)+\sigma_{t} \sigma_{t}^{+} a_{x}(x, t)\right|^{2}}{U_{x x}(x, t)} d t+a(x, t) \cdot d W_{t}, \quad t \geq 0, \quad U(x, 0)=u_{0}(x)
$$

where $\lambda_{t}$ is the market price of risk and $a(x, t)$ is referred to as the volatility of the investment criterion. We motivate this equation and compare it to the corresponding backward SPDE, introduced and studied for the classical problem in [89]. This illustrates the fact that forward criteria are closely related to an inverse formulation of (a stochastic version of) the classical problem. Indeed, within the forward framework, the investor specifies her initial preferences as she acknowledges her commitment to previous investments and the intention to invest in a way which is consistent with those. This comparison, in turn, provides intuition for the fact that whereas the value function for the classical problem is uniquely determined, there exist multiple forward criteria associated with a given initial condition. In particular, there is a one-to-one correspondence between admissible criteria and admissible volatility processes $a(x, t)$ for the SPDE (1.2). We discuss the consequences of this flexibility.

The multiplicity of criteria also allows for the identification of criteria with specific dynamical properties. We discuss two such examples: criteria which are deterministic functions of stochastic factors (studied in [99]) and criteria with zero volatility (i.e. $a(x, t) \equiv 0$, cf. [8, 96]). Crucial for both cases is that the SPDE (1.2) reduces to a (possibly random) PDE. Since there is no volatility component present for the latter, the solution associated with a given initial condition is unique. In particular, the non-volatile criteria turn out to be closely related to the utility functions with completely monotonic inverses studied in Section 2.2. We discuss this connection and, furthermore, relate these criteria to the more general versions of non-volatile forward investmentconsumption and robust criteria studied, respectively, in Sections 3.2 and 4.4 of this thesis.

In Section 3.2, the extended notion of forward investment and consumption criteria is studied. Such a study was initiated in Berrier and Tehranchi [9], where the definition first appeared and where a number of important facts were established. Therein, a general semi-martingale market setting was considered and then, in turn, non-volatile criteria were studied under the assumption 
of a Brownian filtration. Here, we consider a Brownian filtration throughout and the main focus is on establishing explicit characterization and economic interpretation of the non-volatile investment-consumption criteria.

The contribution in Section 3.2 is threefold. First, we consider criteria with non-zero volatility and formally derive the SPDE analogous to (1.2) which they are expected to satisfy. Although only formally derived, this SPDE will illustrate important aspects of the notion and give intuition for the restriction to non-volatile criteria. Second, we provide a detailed study of the non-volatile forward investment-consumption criteria. While some of these results appeared already in [9], we provide the details of the underlying arguments. Third, we provide explicit formulae for the optimal investment and consumption processes. We also show that a specific class of time-monotone preferences, in fact, correspond to a deterministic compilation of classical infinite horizon Merton and pure forward investment criteria. Specifically, we have the following result.

Result (Theorem 3.9 and Propositions 3.13 and 3.14). There is a one-to-one correspondence between non-volatile forward investment consumption criteria and pair of random functions $\left(h, h^{c}\right)$ such that $h(y, t)$ satisfies for all $t \geq 0$ a (backward) heat-equation with source-term given by $h^{c}(y, t)$.

The optimal behaviour (investment strategy and consumption pattern) associated with such a criterion is explicitly given in terms of the pair of functions $\left(h, h^{c}\right)$ and a market related input defined in terms of the market price of risk $\lambda_{t}, t \geq 0$.

Under a specific additional condition, there exists a split of the initial capital such that the same investment behaviour is obtained if the investor separately invests these parts according to, respectively, a standard forward criterion and an infinite horizon Merton criterion.

This result provides economic interpretation and illustrates that forward investment-consumption criteria constitute a generalisation of infinite horizon Merton criteria which allows the investor to consider utility from investments as well as consumption.

\section{Chapter 4}

In Chapter 4, model uncertainty is taken into consideration and the extended notion of robust forward performance criteria is defined. From a decision theoretic point of view, the fact that the model itself might be subject to uncertainty, also referred to as ambiguity or Knightian uncertainty, was addressed in the seminal work by Gilboa and Schmeidler [54]. They formulated axioms on investors' preferences that should account for aversion against both ambiguity and risk. Specifically, within an Anscombe-Aumann model, the axioms of von Neumann and Morgenstern 
were relaxed in that the axiom of independence was replaced by that of certainty independence. That led to a numerical representation of preferences in terms of a coherent monetary utility functional. The robust representation of the latter (cf. [31] for the case of bounded random variables), then implies the following representation of preferences over objects $X$ within some set $\mathcal{X}$ :

$$
X \longmapsto \inf _{\mathbb{Q} \in \mathcal{Q}} \mathrm{E}^{\mathbb{Q}}[U(X)], \quad X \in \mathcal{X},
$$

for some von Neumann Morgenstern utility function $U$ and set $\mathcal{Q}$ of probability measures.

For the case of a fixed finite horizon, this motivated the study of the risk- and ambiguity-averse investor who trades in a continuous-time market model and evaluates terminal wealth according to the criterion (1.3). The investment problem associated with such, so-called multiple-priors, preferences has been well-studied. Stochastic control methods have successfully been applied and explicit solutions obtained for the choice of specific market models and utility functions. Specifically, for stochastic factor models and for possibly non-Markovian models, solutions have been obtained, respectively, in terms of PDE's in [64, 102] and BSDEs in $[93,106]$ (see [46] for a full list of references). For general market models and utility functions, we mention in particular the work by Quenez [106] and Schied and Wu [111]. Relying on the results for the standard utility maximization problem in Kramkov and Schachermayer [79, 80], the authors in [106] and [111] established a dual formulation and proved existence of an optimizer (see also [23, 124] for the case including consumption).

The axiomatic results of Gilboa and Schmeidler were later generalized in Maccheroni et al. [87], where the independence axiom was further relaxed. This led to a numerical representation in terms of a concave monetary utility functional. Combined with the generalisation of the representation of coherent utility functionals to concave ones, for the case of bounded random variables obtained in Föllmer and Schied [44] and Fritelli and Rosazza-Gianin [51], it implied the numerical representation

$$
X \longmapsto \inf _{\mathbb{Q} \in \mathcal{Q}}\left(\mathrm{E}^{\mathbb{Q}}[U(X)]+\gamma(\mathbb{Q})\right),
$$

for some penalty function $\gamma$. While the multiple-priors setup in (1.3) is a worst-case approach, the appearance of $\gamma$ enables the investor to weight the possible market models according to their plausibility. This renders the presentation intuitively appealing.

The investment-problem associated with these so-called variational preferences has also been studied. Particular attention has been paid to the case when the penalty function is given by the relative entropy of $\mathbb{Q}$ with respect to some reference model. Such criteria were introduced already in the seminal work of Hansen and Sargent [3, 58]. For this choice, the problem is most naturally formulated with respect to utility from consumption (or stochastic differential utilities) and the natural tool is the theory of BSDEs. While a systematic study was initiated in [115], 
these results have been considerably extended in a number of articles [18, 26, 40, 69, 86]. For the case of utility from terminal wealth and general variational criteria, stochastic control methods have been successfully applied for the choice of logarithmic utility. This for stochastic factor models as well as non-Markovian ones; see [65, 66] and [82], respectively. General existence and duality results have also been established for the variational preferences. Specifically, by use of similar methods to the ones used in [106, 111], Schied [110] generalised these results to the concave case.

Motivated by these developments, as well as the forward investment criteria discussed in Chapter 3, we combine the notions and define robust forward criteria. While the former notion deals with model uncertainty, the latter addresses issues of horizon and preference ambiguity. The combination of these concepts seems natural and allows the investor to acknowledge her inability to specify exactly the market model as well as the preferences. In the same way as standard forward criteria describe the evolution of value-processes and preferences within a given model, the study of robust forward criteria contribute to our understanding of the evolution of timeconsistent ambiguity averse preferences.

Specifically, we call a utility random field $U(x, t), t \geq 0$, and a family of penalty functions $\gamma_{t, T}$, a robust forward performance criterion if they satisfy the following condition of timeconsistency,

$$
U(\xi, t)=\operatorname{ess}_{\pi \in \mathcal{A}_{t}(\xi)} \underset{\mathbb{Q} \in \operatorname{Qin}_{t}}{\operatorname{ess}}\left\{\mathrm{E}^{\mathbb{Q}}\left[U\left(X_{T}^{\pi}, T\right) \mid \mathcal{F}_{t}\right]+\gamma_{t, T}(\mathbb{Q})\right\}, \quad \xi \in L^{\infty}\left(\mathcal{F}_{t}\right),
$$

for all $0 \leq t \leq T<\infty$. Here $\mathcal{A}_{t}(\xi)$ denotes the set of admissible trading strategies given capital $\xi$ at time $t, X_{T}^{\pi}$ the wealth associated with the strategy $\pi$, and $\mathcal{Q}_{t}$ is the set of (equivalent) market models that the investor considers at time $t$. As for the standard forward criteria, the property of time-consistency is the starting point and the notion characterizes all combinations of stochastic preferences and penalty functions satisfying this property.

In Section 4.1, the main focus is on establishing dual characterizations of the robust forward criteria. Combining ideas developed in [110] and [129], respectively, the following characterization is established:

Result (Theorem 4.6 and Corollary 4.7). Under suitable assumptions, a utility random field $U(x, t)$ constitute a robust forward criterion with respect to a family of penalty functions $\gamma_{t, T}$ if and only if its convex conjugate $V(y, t)$ satisfies

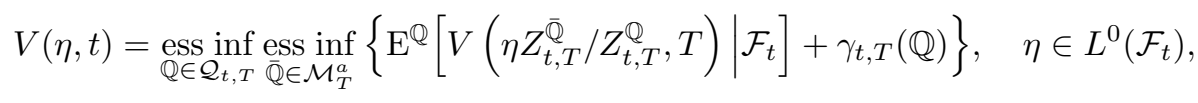

for all $t \leq T<\infty$, where $\mathcal{M}_{T}^{a}$ is the set of absolutely continuous martingale measures. 
Similarly to the classical utility maximization, the study of the problem within the dual domain offers various advantages. Most importantly, in the case of robust preferences the dual problem amounts to a search for an infimum whereas the primal problem features a saddle-point. Study of the dual problem allows us to deduce some further characterizations of time-consistency (cf. Propositions 4.13 and 4.15).

In Section 4.2, we study a specific type of robust forward criteria characterized by a particular dynamic property and referred to as non-volatile criteria; a robust version of the time-monotone criteria studied in $[8,96]$. The discussion, which is formal, aim to illustrate the flexibility of the notion of robust forward criteria and the fact that interesting preferences might be identified under additional evolutionary requirements. In particular, within a Brownian filtration, nonvolatile criteria are linked to a specific PDE (cf. equation (4.34) below) which is discussed in some further detail.

In Section 4.5, a specific non-volatile logarithmic criterion is studied within a Brownian filtration. This criterion is of special interest as it gives rise to an optimal strategy of the following form (cf. Proposition 4.39):

$$
\bar{\pi}_{t}=\frac{\delta_{t}}{1+\delta_{t}} \frac{\hat{\lambda}_{t}}{\sigma_{t}}, \quad t \geq 0
$$

where $\hat{\lambda}_{t}, t \geq 0$, denotes the market price of risk the agent deems most likely and $\delta_{t} \in[0, \infty)$ quantifies his trust in that estimation. That is to say, a fractional Kelly strategy where the fraction to be invested depends on the investor's trust in the estimated Sharpe ratio. The example illustrates that the notion accommodates criteria which produce strategies commonly used in practice. Despite its very specific form, the explicit solution obtained in this case also illustrates a general fact about robust forward criteria. Namely, that for each robust forward investment criterion, there exists a specific (standard) forward criterion in the reference market, which produces the same optimal behaviour. Section 4.5 concludes with a discussion of this property for more general robust forward criteria.

\section{Chapter 5}

In the final chapter, risk- and ambiguity-averse portfolio selection with respect to quasiconcave utility functionals is considered. Here, we focus on the standard set-up when a finite investment horizon is fixed. This work is motivated by the recent developments in Cerreia-Vioglio et al. [25] and, among others, Drapeau and Kupper [35]. In the former, the decision theoretic results in [54] and [87] are extended. Observing that all ambiguity averse preferences are axiomatized by weakening the independence axiom (the coordinate independence axiom within the Savage setting), the authors in [25] take this to its extreme by essentially removing this axiom altogether. 
Thereby, a numerical representation in terms of quasiconcave utility functionals emerges. Recent advances also yield robust representations of the latter; besides [24, 25] and Drapeau and Kupper [35], see also Fritelli and Maggis [49, 50]. This motivates representing preferences via

$$
X \longmapsto \inf _{\mathbb{Q} \in \mathcal{Q}} G\left(\mathbb{Q}, \mathrm{E}^{\mathbb{Q}}[U(X)]\right),
$$

for some function $G$ which is jointly quasiconvex, lower semicontinuous in its first argument and non-decreasing and right-continuous in its second. By use of the same arguments as for the multiple-priors and variational cases (cf. (1.3) and (1.4)), these advances motivate the study of the risk- and ambiguity-averse investor with a criterion defined in terms of a quasi-concave utility functional. The aim in this chapter is to initiate a study of the associated investment problem.

Specifically, we consider an investor who trades over a fixed finite horizon in a continuous-time market model, evaluates terminal wealth according to (1.6) and maximizes this quantity over admissible trading strategies. While the investor's risk-aversion is governed by a standard utility function, the ambiguity preferences are, thus, determined by a quasiconcave utility functional. To the best of our knowledge, this problem has not been studied before. Indeed, although the advances in [25] recently motivated the use of quasiconvex risk measures within the area of portfolio optimization (cf. [90]) and quasiconcave utility functions have been previously studied, the risk- and ambiguity-averse utility maximization problem associated with (1.6) seems to not have been addressed. We note that the notion is unifying in the sense that all ambiguity averse preferences, in particular the multiple-priors, entropic and variational ones, are included as special cases. The class of quasiconcave preferences also includes interesting examples which do not correspond to variational preferences. Among others, the so-called smooth preferences axiomatized in [77], which amount to considering a distribution over possible distributions rather than a worst-case approach.

Our results extend the ones in [110] (cf. also $[106,111]$ ) to the quasiconcave case. We prove existence of an optimal strategy and establish certain duality results. Specifically, we obtain the following:

Result (Theorems 5.5, 5.9 and 5.10). Under suitable assumptions, there exists an optimal strategy with associated terminal payoff $\bar{X}_{T}$. Moreover,

$$
u(x):=\sup _{X \in \mathcal{X}(x)} \inf _{\mathbb{Q} \in \mathcal{Q}} G\left(\mathbb{Q}, \mathrm{E}^{\mathbb{Q}}\left[U\left(X_{T}\right)\right]\right)=\inf _{y>0} v(y ; x),
$$

where the dual value function is given by

$$
v(y ; x)=\inf _{\mathbb{Q} \in \mathcal{Q} Y \in \mathcal{Y}(y)} G\left(\mathbb{Q}, \mathrm{E}\left[Z^{\mathbb{Q}} V\left(Y_{T} / Z^{\mathbb{Q}}\right)\right]+x y\right),
$$


where $\mathcal{X}(x)$ is the set of admissible wealth processes and $\mathcal{Y}(y)$ the associated set of dual objects. Moreover, the dual problem admits a solution $(\bar{Y}, \overline{\mathbb{Q}})$ such that $(\bar{X}, \overline{\mathbb{Q}})$ is a saddle point for the primal problem and

$$
\bar{X}_{T}=\left(U^{\prime}\right)^{(-1)}\left(\bar{Y}_{T} / \bar{Z}\right), \quad \overline{\mathbb{Q}} \text {-a.s. }
$$

We note that the proofs in [110] make use of certain limiting arguments, by which the set appearing in (1.4) is reduced to a set of measures equivalent to $\mathbb{P}$, for which the results in $[79,80]$ can be directly applied. However, due to the quasi-concavity, as opposed to the concavity, of the utility functional, we were not able to apply this approach in our case. Our proofs are rather based on the fact that the duality results in $[79,80]$ can be verified to hold also for a specific auxiliary optimization problem. In particular, we obtain alternative proofs of some of the results in [110]. 


\section{Chapter 2}

\section{New insights for optimal portfolios and risk preferences when investing in log-normal markets}

\subsection{Qualitative analysis of optimal investment policies and robustness of risk tolerance}

We study qualitative properties of the optimal investment strategies and other related quantities of a risk averse investor who trades in a finite horizon and aims to maximize her expected utility from terminal wealth.

Trading takes place among a riskless bond and $N$ stocks whose prices are log-normally distributed. This optimal investment problem, introduced by Merton [91], is readily solved either by using duality and related martingale analysis, or PDE techniques applied to the associated Hamilton-Jacobi-Bellman equation. In the latter case, the first order conditions yield the socalled optimal feedback controls which, in turn, generate the optimal investment processes.

Despite the simplicity of both the market setting and the underlying stochastic optimization problem, as well as the popularity of the latter and its important extensions in the academic investment literature, relatively little is known about the qualitative behavior of the optimal strategies, except when the utility is homothetic. In this case, the optimal portfolios are linear functions of the current wealth and independent of time, and thus their structure can be easily analyzed.

Our aim herein is to consider general utility functions and carry out a detailed qualitative study 
for the optimal feedback portfolio functions and optimal weights, as well as of the local risk aversion and risk tolerance coefficients. Some of the properties we analyze have been studied before, either in discrete or continuous-time settings, but we use an alternative methodology which yields much shorter and direct proofs. Some other properties we consider are new. A byproduct of our work is the compilation of a concise study with old and new results of the optimal strategies and related quantities. We discuss the questions we consider next.

- When are the optimal allocations and optimal weights increasing in the wealth argument?

For general risk preferences, this question was examined by Arrow [5] who showed that, in a single period problem with one risky stock, the optimal investment in the latter is increasing in wealth if and only if the investor's utility exhibits decreasing absolute risk aversion $(D A R A)$, and as long as the risk premium is positive. He, also, showed that the fraction of wealth invested in the stock, known as the average propensity to invest, is decreasing in wealth if and only if the utility exhibits increasing relative risk aversion (IRRA). Since this seminal work, it has become common in the economic literature to assume that the utility exhibits the DARA and IRRA; these properties are also known as the Arrow hypothesis.

Borell [19] is, to our knowledge, the first who studied the above questions in a continuous-time setting. The main result in [19] is that, in the log-normal model, if the investor's risk aversion coefficient is decreasing in wealth, then this spatial monotonicity is inherited at all trading times to both the absolute and relative local risk aversion functions and, in turn, to the optimal portfolios and optimal weights. The same results were obtained using a different methodology by Xia in [125]. Herein, we also study this question and provide a much shorter proof, alternative to the ones in [19] and [125].

- When are the optimal allocations convex/concave in the wealth argument?

The concavity of the optimal portfolios is related to the so-called marginal propensity to invest $(M P I)$, which is defined as the derivative of the optimal portfolio with respect to wealth. Then, $M P I$ is decreasing (increasing) in wealth if and only if the optimal portfolio function is concave (convex) in wealth. We show that if the risk tolerance is a strictly concave/convex function then, the same property is inherited by the optimal portfolio at all trading times.

- When are the optimal allocations and optimal weights increasing/decreasing with respect to the time?

Very little is known about this important property in continuous time settings. While it was not, naturally, an issue in single-period models, it has not received appropriate attention even in discrete-settings. To our knowledge, Gollier and Zeckhauser [55] are the only ones who have 
studied it in a multi-period setting. They investigated the connection between horizon length/age of investors and curvature of their absolute risk aversion.

Herein, we prove similar results in the continuous-time setting. We show that if the absolute risk tolerance coefficient is a concave/convex function of wealth, then the optimal feedback allocations and optimal weights are always increasing/decreasing as time increases. The proof of this surprising finding is a mere direct consequence of the concavity/convexity properties mentioned earlier. The temporal behaviour of the optimal policies have also been examined in more extended continuous time model settings in [27] and [84]. Therein, however, the generality of the model did not allow for specific results as the one herein.

- Are optimal allocations and optimal weights robust with respect to risk preferences?

The question whether two individuals with ordered risk aversion coefficients invest in an analogous way at all trading times has been analyzed extensively in single- and multi-period settings. In continuous time, such a result was established in [125] for log-normal prices. Herein, we provide a much shorter proof of this result. While the result holds for a slightly smaller class of solutions than the one in [125], it bypasses several lengthy penalization and approximation arguments, and is based on entirely different methodology. The arguments are short and direct and are, in turn, used to derive robust bounds for the optimal policies, and the local risk tolerance and risk aversion coefficients.

- How does the optimal allocation, local risk tolerance and local risk aversion functions depend on the market parameters?

It follows easily that all the above monotonicity, concavity and robustness results hold for both the optimal investments and the local risk tolerance and risk aversion functions as these quantities are mere scalar multiples of each other (see (2.13) and (2.15)). This is not, however, the case for the dependence of these quantities on market coefficients, for the latter appear rather implicitly in the relevant formulae. We examine this dependence. For the case of one stock, we find, for example, that while the optimal feedback portfolio function is always decreasing in the stock's volatility, the analogous monotonicity of the local absolute risk tolerance depends exclusively on the spatial convexity/concavity of the risk tolerance coefficient.

The aforementioned continuous-time results in [19] and [125] were proved via martingale methods applied to the dual problem. Herein, we use throughout a different methodology which relies on properties of solutions of auxiliary linear and nonlinear partial differential equations. The nonlinear equations are satisfied by the local risk tolerance function, the local risk aversion and the optimal feedback strategies. A key transformation, see (2.31), relates their solutions to the ones of the heat equation and its derivatives. Several properties are then established by looking at how 
analogous properties of the terminal data are propagated at earlier times. The robustness, the related bounds, and the dependence on market parameters are derived by applying appropriate comparison results.

One should note, however, that all these approaches have similar characteristics as they focus on the dual and not the primal problem. The pde methodology used herein, however, allows for substantially more tractability, makes the comparison arguments more transparent and requires much shorter technical arguments. More importantly, it enables us to analyze the time behavior and the dependence of the optimal portfolios on market parameters.

A by-product of our methodology is the construction of alternative representations for the optimal portfolio and wealth processes through the underlying auxiliary harmonic functions. These stochastic representations are, naturally, equivalent to the ones derived by duality arguments but yield more direct and easier to handle formulae. Using these expressions, we readily derive the sensitivities of the optimal processes with respect to initial wealth.

The remainder of this section is organized as follows. In Section 2.1.1 we introduce the investment model and review the classical results. In Section 2.1.2 we derive the auxiliary PDE and provide background results, and also establish alternative stochastic representations for the optimal processes. In Section 2.1.3 we examine the monotonicity - spatial and temporal - and the concavity of the optimal portfolios. In Section 2.1.4, we establish the robustness results and provide related bounds. In Section 2.1.5, we examine the sensitivities of the optimal portfolios, and of the local risk tolerance and risk aversion functions with respect to market parameters, as well as the sensitivity of the optimal wealth and portfolio processes with respect to initial wealth.

\subsubsection{The log-normal investment model}

We briefly recall the classical Merton problem (cf. [91]), its value function and optimal policies. Trading takes place in $[0, T]$, with the horizon $T$ being arbitrary but fixed.

The market environment consists of one riskless and $N$ risky securities. The risky securities are stocks and their prices are modelled as log-normal processes. Namely, for $i=1, \ldots, N$, the price $S_{t}^{i}, 0 \leq t \leq T$, of the $i^{t h}$ risky asset satisfies

$$
d S_{t}^{i}=S_{t}^{i}\left(\mu^{i} d t+\sum_{j=1}^{N} \sigma^{j i} d W_{t}^{j}\right),
$$

with $S_{0}^{i}>0$, for $i=1, \ldots, N$. The process $W_{t}=\left(W_{t}^{1}, \ldots, W_{t}^{N}\right), t \geq 0$, is a standard $N$-dimensional Brownian motion, defined on a filtered probability space $(\Omega, \mathcal{F}, \mathbb{P})$. For simplicity, it is assumed 
that the underlying filtration, $\mathcal{F}_{t}$, coincides with the one generated by the Brownian motion, that is $\mathcal{F}_{t}=\sigma\left(W_{s}: 0 \leq s \leq t\right)$.

The coefficients $\mu^{i}$ and $\sigma^{i}=\left(\sigma_{t}^{1 i}, \ldots, \sigma^{N i}\right), i=1, \ldots, N, t \geq 0$, are constants with values in $\mathbb{R}$ and $\mathbb{R}^{N}$, respectively. For brevity, we use $\sigma$ to denote the $N \times N$ matrix volatility $\left(\sigma^{j i}\right)$, whose $i^{t h}$ column represents the volatility $\sigma^{i}=\left(\sigma^{1 i}, \ldots, \sigma^{N i}\right)$ of the $i^{\text {th }}$ risky asset. Alternatively, we write $(2.1)$ as

$$
d S_{t}^{i}=S_{t}^{i}\left(\mu^{i} d t+\sigma^{i} \cdot d W_{t}\right)
$$

The riskless asset, the savings account, offers constant interest rate $r>0$. We denote by $\mu$ the $N \times 1$ vector with coordinates $\mu^{i}$ and by $\mathbf{1}$ the $N$-dimensional vector with every component equal to one.

We assume that the volatility matrix is invertible, and define the vector

$$
\lambda=\left(\sigma^{T}\right)^{-1}(\mu-r \mathbf{1}) .
$$

It is throughout assumed that all entries of $\sigma^{-1} \lambda$ are positive. This assumption is imposed for mere convenience so that all the properties of the optimal portfolios and the local risk toelrance are aligned (see, for example, Remark 2.10).

Starting at $t \in[0, T)$ with an initial endowment $x>0$, the investor invests at any time $s \in(t, T]$ in the riskless and risky assets. The present value of the amounts invested are denoted, respectively, by $\pi_{s}^{0}$ and $\pi_{s}^{i}, i=1, \ldots, N$.

The present value of her investment is, then, given by $X_{s}^{\pi}=\sum_{k=0}^{N} \pi_{t}^{k}, s \in(t, T]$. We will refer to $X^{\pi}$ as the discounted wealth generated by the strategy $\left(\pi_{s}^{0}, \pi_{s}^{1}, \ldots, \pi_{s}^{N}\right)$. The investment strategies will play the role of control processes and are taken to be self-financing. We easily deduce that the discounted wealth satisfies

$$
d X_{s}^{\pi}=\sigma \pi_{s} \cdot\left(\lambda d s+d W_{s}\right)
$$

with initial wealth $X_{t}=x$, and where the (column) vector, $\pi_{s}=\left(\pi_{s}^{i} ; i=1, \ldots, N\right)$. The investment process, $\pi_{s}$ is admissible if $\pi_{s} \in \mathcal{F}_{s}, E\left(\int_{t}^{T}\left|\pi_{s}\right|^{2} d s\right)<\infty$ and the associated wealth remains non-negative, $X_{s}^{\pi} \geq 0,0 \leq t \leq s \leq T$. We denote the set of admissible strategies by $\mathcal{A}$.

The investor's utility function at $T$ is given by $U: \mathbb{R}_{+} \rightarrow \mathbb{R}$, and it is assumed to be a strictly concave, strictly increasing and $C^{4}(0, \infty)$ function, satisfying the usual Inada conditions

$$
\lim _{x \rightarrow 0} U^{\prime}(x)=+\infty \quad \text { and } \quad \lim _{x \rightarrow \infty} U^{\prime}(x)=0 .
$$

We recall the inverse of the marginal utility $U^{\prime}, I: \mathbb{R}_{+} \rightarrow \mathbb{R}_{+}$, 


$$
I(x)=\left(U^{\prime}\right)^{(-1)}(x)
$$

and assume that, for some $\gamma>0$, it satisfies the polynomial growth condition

$$
I(x) \leq \gamma+x^{-\gamma}
$$

The value function is defined as the maximal expected utility,

$$
u(x, t)=\sup _{\pi \in \mathcal{A}} E_{\mathbb{P}}\left(U\left(X_{T}^{\pi}\right) \mid X_{t}^{\pi}=x\right),
$$

where $X_{s}^{\pi}, s \in(t, T]$, solves $(2.3)$.

The above stochastic optimization problem has been widely studied and completely solved. We provide the main results below without a proof (see, for example, [13] and [72]).

Proposition 2.1. i) The value function $u \in C^{4,1}\left(\mathbb{R}_{+} \times[0, T]\right)$ is strictly increasing and strictly concave in the spatial variable, and solves the Hamilton-Jacobi-Bellman (HJB) equation,

$$
u_{t}+\frac{1}{2}|\lambda|^{2} \frac{u_{x}^{2}}{u_{x x}}=0
$$

with $u(x, T)=U(x)$, with $\lambda$ as in (2.2).

ii) The optimal portfolio process is given, for $s \in[t, T]$, by

$$
\pi_{s}^{*}=\pi^{*}\left(X_{s}^{*}, s\right)=\left(\pi^{1, *}\left(X_{s}^{*}, s\right), \ldots, \pi^{N, *}\left(X_{s}^{*}, s\right)\right),
$$

where the optimal feedback portfolio vector $\pi^{*}: \mathbb{R}_{+} \times[0, T] \rightarrow \mathbb{R}_{+}^{N}$ is given by

$$
\pi^{*}(x, t)=-\frac{u_{x}(x, t)}{u_{x x}(x, t)} \sigma^{-1} \lambda,
$$

and $X_{s}^{*}, s \in[t, T]$, is the optimal wealth process solving (2.3) with $\pi_{s}^{*}$ given in (2.9) being used. Associated with any utility function are the absolute risk tolerance and absolute risk aversion coefficients, denoted by the functions $R(x)$ and $A(x)$, given respectively, for $x \geq 0$, by

$$
R(x)=-\frac{U^{\prime}(x)}{U^{\prime \prime}(x)} \quad \text { and } \quad A(x)=-\frac{U^{\prime \prime}(x)}{U^{\prime}(x)} .
$$

Standing assumptions for the risk tolerance coefficient is that $R(0)=0$ and that $R(x)$ is strictly increasing for $x \geq 0$, previously mentioned as the $D A R A$ hypothesis.

The relative risk tolerance and relative risk aversion coefficients, denoted by the functions $\tilde{R}(x)$ and $\tilde{A}(x)$, are the normalized by wealth analogues of $(2.11)$,

$$
\tilde{R}(x)=-\frac{U^{\prime}(x)}{x U^{\prime \prime}(x)} \quad \text { and } \quad \tilde{A}(x)=-\frac{x U^{\prime \prime}(x)}{U^{\prime}(x)} .
$$


For intermediate trading times $t \in[0, T)$, one then defines the associated local, or indirect, absolute and relative coefficients. The local absolute risk tolerance, $r(x, t)$, and the local absolute risk aversion, $\gamma(x, t)$, are defined on $\mathbb{R}_{+} \times[0, T)$ and given, respectively, by

$$
r(x, t)=-\frac{u_{x}(x, t)}{u_{x x}(x, t)} \quad \text { and } \quad \gamma(x, t)=-\frac{u_{x x}(x, t)}{u_{x}(x, t)},
$$

with $u$ being the value function, while the corresponding local relative risk tolerance, $\tilde{r}(x, t)$, and local relative risk aversion, $\tilde{\gamma}(x, t)$, are given, respectively, by

$$
\tilde{r}(x, t)=\frac{r(x, t)}{x} \quad \text { and } \quad \tilde{\gamma}(x, t)=\frac{\gamma(x, t)}{x} .
$$

It is then immediate from (9), (12) and (13) that the optimal portfolio, $\pi_{s}^{*}$, and optimal weight processes, denoted by $\tilde{\pi}_{s}^{*}$, are given, for $s \in[t, T]$, respectively, by

$$
\pi_{s}^{*}=r\left(X_{s}^{*}, s\right) \sigma^{-1} \lambda \quad \text { and } \quad \tilde{\pi}_{s}^{*}=\frac{r\left(X_{s}^{*}, s\right)}{X_{s}^{*}} \sigma^{-1} \lambda .
$$

\subsubsection{Auxiliary equations, harmonic functions and feedback controls}

We start our study considering two auxiliary partial differential equations which we use throughout. The first is a known fast-diffusion type equation that the local absolute risk tolerance and the optimal portfolios solve (cf. (2.16) and (2.18)). The second is the classical heat equation satisfied by a function, denoted by $H$, which is defined in (2.21) and linked with the local risk tolerance function as in (2.31).

The function $H$ plays a pivotal role in the analysis herein, for the various derivatives of the local risk tolerance and the optimal feedback portfolio functionals reduce to rather simple expressions involving only its spatial derivatives. These derivatives also solve the heat equation, and this equation admits comparison principle. As a result, various complicated comparison results, which are needed for proving the desired monotonicity, robustness, and sensitivity properties reduce to direct comparisons of solutions to the involved heat equations.

The function $H$ also helps us derive stochastic representations of the optimal wealth and portfolio processes. They are provided in (2.32) and (2.33) and are, to the best of our knowledge, new.

We note that the transformation (2.21) is a mere variation of the Fenchel-Legendre transformation which has been widely used for the analysis of the Merton problem. Moreover (see Proposition 2.7), the alternative stochastic representations (2.32) and (2.33) are also variations of known stochastic expressions derived from duality theory. However, the representations we 
use herein turn out to be quite convenient for the analysis and study of various complicated nonlinear expressions of interest, which enables us to derive results we did not know before.

Proposition 2.2. i) The local absolute risk tolerance function $r(x, t)$ (cf. (2.13)) satisfies, for $(x, t) \in \mathbb{R}_{+} \times(0, T]$, the equation

$$
r_{t}+\frac{1}{2}|\lambda|^{2} r^{2} r_{x x}=0
$$

with

$$
r(x, T)=R(x) .
$$

ii) The local absolute risk aversion function $\gamma(x, t)$ (cf. (2.13)) satisfies satisfies, for $(x, t) \in$ $\mathbb{R}_{+} \times(0, T]$, the equation

$$
\gamma_{t}=\frac{1}{2}|\lambda|^{2}\left(\frac{1}{\gamma}\right)_{x x}
$$

with

$$
\gamma(x, T)=A(x) .
$$

iii) Each component, $\pi^{*, i}, i=1, \ldots, N$, of the optimal feedback portfolio vector $\pi^{*}$ solves, for $(x, t) \in \mathbb{R}_{+} \times(0, T]$,

$$
\pi_{t}^{*, i}+\frac{1}{2} m_{i}^{2}\left(\pi^{*, i}\right)^{2} \pi_{x x}^{*, i}=0
$$

with

$$
\pi^{*, i}(x, T)=k_{i} R(x) .
$$

where $k_{i}$ is the $i^{\text {th }}$ element of the vector $\sigma^{-1} \lambda$ and $m_{i}=k_{i}^{-1}|\lambda|$.

iii) For each $i=1, \ldots N$, and $t \in[0, T]$,

$$
\lim _{x \rightarrow 0} \pi^{*, i}(x, t)=\lim _{x \rightarrow 0} r(x, t)=0 .
$$

The derivation of (2.18) can be found, among others, in [59], [68] and [125]; see, also, the old note of Black [15]. Equations (2.16) follow trivially from (2.10) and (2.18). Finally, assertion (2.20) is well known; it can be proved, for example, by sending $x \rightarrow 0$ in equalities (3.2) and (3.3) in [125].

Lemma 2.3. Let $I: \mathbb{R}_{+} \rightarrow \mathbb{R}_{+}$be given by (2.5) and assume that it satisfies the growth condition (2.6). Let, also, $H: \mathbb{R} \times[0, T] \rightarrow \mathbb{R}_{+}$be defined by

$$
u_{x}(H(x, t), t)=\exp \left(-x-\frac{1}{2}|\lambda|^{2}(T-t)\right)
$$

where $u(x, t)$ is the value function. Then, 
i) the function $H(x, t)$ solves the heat equation,

$$
H_{t}+\frac{1}{2}|\lambda|^{2} H_{x x}=0
$$

with terminal condition

$$
H(x, T)=I\left(e^{-x}\right)
$$

ii) For each $t \in[0, T], H(x, t)$ is strictly increasing and of full range,

$$
\lim _{x \rightarrow-\infty} H(x, t)=0 \quad \text { and } \quad \lim _{x \rightarrow \infty} H(x, t)=\infty .
$$

Proof. The fact that $H$ solves the heat equation follows directly from (2.8) and (2.21). The existence and uniqueness of solutions to (2.22) follows directly from (2.6) and (2.23) (see, for example, [123]). The monotonicity assertion follows from the fact that the function $H_{x}(x, t)$ solves the heat equation with positive terminal condition, $H_{x}(x, T)=-e^{-x} I^{\prime}\left(e^{-x}\right)>0$, and standard comparison arguments (see, for example, [105] and [123]). To show (2.24), we recall that the value function $u(x, t)$ satisfies, for each $t \in[0, T]$ the Inada conditions (2.4) (see, for example, [72]), and (2.24) follows from (2.21).

Besides the comparison results for solutions of the heat equation, which we do not list, we will be using two other results, namely, the preservation of the log-convexity and log-concavity of its solutions. The log-convexity property is a mere consequence of Hölder's inequality while the log-concavity is more involved. For the latter, we refer the reader to Theorem 1.3 in [21] or to [20] and, for the case with boundary data, to [75]. We note, however, that for the one-dimensional case we consider, also the preservation of log-concavity is quite straight-forward. It was first proven in [112]. For the reader's convenience, we next state these two properties with proofs.

Proposition 2.4. Let $h: \mathbb{R} \times[0, T] \rightarrow \mathbb{R}_{+}$be the solution of the heat equation

$$
h_{t}+\frac{1}{2}|\lambda|^{2} h_{x x}=0
$$

with terminal data $h(x, T)=h_{0}(x)$, with $h_{0} \in \mathcal{C}^{2}(\mathbb{R})$ satisfying the growth assumption $h_{0}(x) \leq$ $\gamma+e^{\gamma x}, x \in \mathbb{R}$. If $h_{0}(x)$ is a log-convex function, then for $(x, t) \in \mathbb{R} \times[0, T), h(x, t)$ is also log-convex.

Proof. For simplicity, we set $|\lambda|^{2}=1$. We need to show that for $\alpha \in(0,1)$ and $(x, t) \in \mathbb{R} \times[0, T]$,

$$
h(\alpha x+(1-\alpha) y, t) \leq h(x, t)^{\alpha} h(y, t)^{1-\alpha} .
$$

The Feynman-Kac formula, the log-convexity of the terminal datum and Hölder's inequality yield

$$
h(\alpha x+(1-\alpha) y, t)=E\left(h_{0}\left(\alpha\left(x+W_{T-t}\right)+(1-\alpha)\left(y+W_{T-t}\right)\right)\right)
$$




$$
\begin{gathered}
\leq E\left(\left(h_{0}\left(x+W_{T-t}\right)\right)^{\alpha}\left(h_{0}\left(y+W_{T-t}\right)\right)^{1-\alpha}\right) \\
\leq\left(E\left(h_{0}\left(x+W_{T-t}\right)\right)\right)^{\alpha}\left(E\left(h_{0}\left(y+W_{T-t}\right)\right)\right)^{1-\alpha} \\
=(h(x, t))^{\alpha}(h(y, t))^{1-\alpha} .
\end{gathered}
$$

Proposition 2.5. Let $h: \mathbb{R} \times[0, T] \rightarrow \mathbb{R}_{+}$be the solution of the heat equation

$$
h_{t}+\frac{1}{2}|\lambda|^{2} h_{x x}=0
$$

with terminal data $h(x, T)=h_{0}(x)$, with $h_{0} \in \mathcal{C}^{2}(\mathbb{R})$ satisfying the growth assumption $h_{0}(x) \leq$ $\gamma+e^{\gamma x}, x \in \mathbb{R}$. If $h_{0}(x)$ is a log-concave function, then for $(x, t) \in \mathbb{R} \times[0, T), h(x, t)$ is also log-concave.

Proof. For simplicity, we set $|\lambda|^{2}=1$. We need to show that for $\alpha \in(0,1)$ and $(x, t) \in \mathbb{R} \times[0, T]$,

$$
h(\alpha x+(1-\alpha) y, t) \geq h(x, t)^{\alpha} h(y, t)^{1-\alpha} .
$$

The Prékopa-Leindler inequality yields that, if for $0<\alpha<1, z, z^{\prime} \in \mathbb{R}$, the positive functions $f, m, n$ satisfy, for $z \in \mathbb{R}$,

$$
f\left(\alpha z+(1-\alpha) z^{\prime}\right) \geq(m(z))^{\alpha}\left(n\left(z^{\prime}\right)\right)^{1-\alpha},
$$

then

$$
\int_{-\infty}^{\infty} f(z) d z \geq\left(\int_{-\infty}^{\infty} m(z) d z\right)^{\alpha}\left(\int_{-\infty}^{\infty} n(z) d z\right)^{1-\alpha}
$$

If the terminal datum, $h_{0}(x)$, is log-concave, then, for $\alpha \in(0,1), z, z^{\prime} \in \mathbb{R}$,

$$
h_{0}\left(\alpha z+(1-\alpha) z^{\prime}\right) \geq\left(h_{0}(z)\right)^{\alpha}\left(h_{0}\left(z^{\prime}\right)\right)^{1-\alpha} .
$$

Next, fix $(x, y, t) \in \mathbb{R} \times \mathbb{R} \times[0, T]$ and define the functions

$$
\begin{gathered}
f(z ; x, y, t)=e^{-\left(\frac{\alpha x+(1-\alpha) y-z}{4(T-t)}\right)^{2}} h_{0}(z) \\
m(z ; x, t)=e^{-\left(\frac{x-z}{4(T-t)}\right)^{2}} h_{0}(z) \quad \text { and } n(z ; y, t)=e^{-\left(\frac{y-z}{4(T-t)}\right)^{2}} h_{0}(z) .
\end{gathered}
$$

We easily see, that

$$
f\left(\alpha z+(1-\alpha) z^{\prime} ; x, y, t\right) \geq(m(z ; x, t))^{\alpha}\left(n\left(z^{\prime} ; y, t\right)\right)^{1-\alpha} .
$$

Indeed, from the log-concavity of the functions $h_{0}(x)$ and $e^{-x^{2}}$ we have

$$
f\left(\alpha z+(1-\alpha) z^{\prime} ; x, y, t\right)=e^{-\left(\frac{\alpha x+(1-\alpha) y-\alpha z-(1-\alpha) z^{\prime}}{4(T-t)}\right)^{2}} h_{0}\left(\alpha z+(1-\alpha) z^{\prime}\right)
$$




$$
\begin{aligned}
& \geq e^{-\left(\frac{\alpha(x-z)+(1-\alpha)\left(y-z^{\prime}\right)}{4(T-t)}\right)^{2}}\left(h_{0}(z)\right)^{\alpha}\left(h_{0}\left(z^{\prime}\right)\right)^{1-\alpha} \\
\geq & \left(e^{-\left(\frac{x-z}{4(T-t)}\right)^{2}} h_{0}(z)\right)^{\alpha}\left(e^{-\left(\frac{y-z^{\prime}}{4(T-t)}\right)^{2}} h_{0}\left(z^{\prime}\right)\right)^{1-\alpha} .
\end{aligned}
$$

Therefore, inequality (2.28) yields

$$
\int_{-\infty}^{\infty} e^{-\left(\frac{\alpha x+(1-\alpha) y-z}{4(T-t)}\right)^{2}} h_{0}(z) d z \geq\left(\int_{-\infty}^{\infty} e^{-\left(\frac{x-z}{4(T-t)}\right)^{2}} h_{0}(z) d z\right)^{\alpha}\left(\int_{-\infty}^{\infty} e^{-\left(\frac{y-z}{4(T-t)}\right)^{2}} h_{0}(z) d z\right)^{1-\alpha}
$$

and we easily obtain that the solution $h(x, t)$ of $(2.26)$ satisfies

$$
h(\alpha x+(1-\alpha) y, t) \geq(h(x, t))^{\alpha}(h(y, t))^{1-\alpha} .
$$

As mentioned earlier, the next result is pivotal for the analysis herein and will be used repeatedly throughout. It follows directly from (2.13) and (2.21), and provides a useful representation of the local risk tolerance function via the spatial inverse of the auxiliary harmonic function and its first spatial derivative.

Lemma 2.6. The local absolute risk tolerance function $r(x, t)$ is given, for $(x, t) \in \mathbb{R}_{+} \times(0, T]$, by

$$
r(x, t)=H_{x}\left(H^{(-1)}(x, t), t\right),
$$

where $H$ satisfies (2.22) and (2.23).

\subsubsection{The optimal portfolio and wealth processes}

We provide representation results for the optimal wealth and portfolio processes. For simplicity, we assume that the initial time is $t=0$. To our knowledge, the following expressions are new for the Merton problem.

Proposition 2.7. The optimal wealth $X_{t}^{*}, t \in[0, T]$, starting at $x$ at time 0 , and the associated optimal investment vector $\pi_{t}^{*}$ are given, respectively, by the processes

$$
X_{t}^{*}=H\left(H^{(-1)}(x, 0)+|\lambda|^{2} t+\lambda \cdot W_{t}, t\right)
$$

and

$$
\begin{gathered}
\pi_{t}^{*}=H_{x}\left(H^{(-1)}\left(X_{t}^{*}, t\right), t\right) \sigma^{-1} \lambda \\
=H_{x}\left(H^{(-1)}(x, t)+|\lambda|^{2} t+\lambda \cdot W_{t}, t\right) \sigma^{-1} \lambda,
\end{gathered}
$$

where $H: \mathbb{R} \times[0, T] \rightarrow \mathbb{R}^{+}$satisfies (2.22) and (2.23). 
Proof. Assertion (2.33) is derived from (2.15) and (2.31). In turn, (2.34) follows from (2.33) and (2.32). Therefore, it remains to show (2.32).

For this, define the auxiliary process $N_{t}=H^{(-1)}(x, 0)+|\lambda|^{2} t+\lambda \cdot W_{t}$ and apply Ito's formula to the process $H\left(N_{t}, t\right)$. We then have that

$$
\begin{gathered}
d H\left(N_{t}, t\right)=H_{x}\left(N_{t}, t\right) d N_{t}+\frac{1}{2} H_{x x}\left(N_{t}, t\right) d\langle N\rangle_{t}+H_{t}\left(N_{t}, t\right) d t \\
=H_{x}\left(N_{t}, t\right)\left(|\lambda|^{2} d t+\lambda \cdot d W_{t}\right)+\left(H_{t}\left(N_{t}, t\right)+\frac{1}{2}|\lambda|^{2} H_{x x}\left(N_{t}, t\right)\right) d t \\
=H_{x}\left(N_{t}, t\right)\left(|\lambda|^{2} d t+\lambda \cdot d W_{t}\right),
\end{gathered}
$$

where we used that $H$ solves (2.22). We easily deduce that the process $H\left(N_{t}, t\right)$ is the optimal wealth generated by $\pi_{t}^{*}$ and we conclude.

We remark that one could use standard duality results to derive the above formulae. The construction is in reverse order, in that the wealth representation (2.32) is established first and then (2.33) follows from a direct application of Ito's formula and (2.3). This is highlighted below.

Remark 2.8. Let $Z_{T}$ be the Radon-Nikodym derivative of the unique martingale measure, say $\mathbb{Q}$, with respect to the historical measure $\mathbb{P}$. Let also $\xi^{x} \in \mathbb{R}_{+}$be the optimal Lagrange multiplier satisfying the budget constraint $E_{\mathbb{P}}\left(Z_{T} I\left(\xi^{x} Z_{T}\right)\right)=x$, with $I$ as in (2.5). Then, we have that $\xi^{x}=\exp \left(-H^{(-1)}(x, 0)-\frac{1}{2}|\lambda|^{2} T\right)$. In turn, for $t \in[0, T]$, the optimal wealth process is given by

$$
X_{t}^{*}=E_{\mathbb{Q}}\left(I\left(\xi^{x} Z_{T}\right) \mid \mathcal{F}_{t}\right)=H\left(H^{(-1)}(x, 0)+|\lambda|^{2} t+\lambda \cdot W_{t}, t\right),
$$

with $H(x, t)$ satisfying (2.22) and (2.23).

\subsubsection{Spatial and temporal properties of optimal allocations and opti- mal weights}

We study the questions stated above on the monotonicity and concavity of the optimal allocations and optimal weights with respect to wealth, as well as their monotonicity with respect to time. Because these quantities are affine transformations of the local absolute and relative risk tolerances (cf. (2.15)), we only provide the related proofs for the latter. For completeness though, we state each result for all related quantities. 


\subsubsection{Monotonicity in wealth}

We start with the monotonicity properties of the optimal allocations and optimal weights. We recall the standing assumption that the risk tolerance coefficient $R(x)$ is strictly increasing for $x \geq 0$. As mentioned earlier, these properties were first established for the log-normal model in [19] and, more recently, in [125]. Below, we provide alternative short proofs to these results.

Proposition 2.9. Let the absolute risk tolerance coefficient $R(x), x \geq 0$, be strictly increasing. Then, for each $t \in[0, T)$ and $x \geq 0$,

i) the optimal allocations $\pi^{*, i}(x, t), x \geq 0, i=1, \ldots, N$, are strictly increasing.

ii) the local absolute risk tolerance and risk aversion coefficients $r(x, t)$ and $\gamma(x, t)$ are, respectively, strictly increasing and strictly decreasing.

Proof. Differentiating (2.31) for $t=T$ yields,

$$
R^{\prime}(x)=\left.\frac{H_{x x}(z, T)}{H_{x}(z, T)}\right|_{z=H^{(-1)}(x, T)} .
$$

Using the monotonicity of $H(x, t)$ and $(2.24)$, we see that $R(x)$ is strictly increasing if and only if the auxiliary function $H(x, T)$ is strictly concave, $H_{x x}(x, T)>0$.

On the other hand, the function $H_{x x}(x, t)$ also solves the heat equation (2.22) with terminal condition $H_{x x}(x, T)=\left(I\left(e^{-x}\right)\right)^{\prime \prime}$. Using (2.6), we easily deduce that the terminal condition $H_{x x}(x, T)$ satisfies the appropriate conditions for existence and uniqueness of solutions. A straightforward application of the comparison principle then yields that $H_{x x}(x, t)>0$, for $(x, t) \in \mathbb{R} \times[0, T)$. Using $(2.31)$ once more, we have that

$$
r_{x}(x, t)=\left.\frac{H_{x x}(z, t)}{H_{x}(z, t)}\right|_{z=H^{(-1)}(x, t)},
$$

and using that $H_{x}(x, t)>0$ (see part (ii) in Lemma 2.3) we easily conclude.

Remark 2.10. In Section 2.1.1, it was assumed that all entries of the vector $\sigma^{-1} \lambda$ are positive. As the above proof shows, this was made for mere convenience, so that the signs of the risk tolerance and the optimal feedback portfolios are the same. The results in the above proposition and in all subsequent ones can be readily generalized if the elements of $\sigma^{-1} \lambda$ are of different signs.

We continue with the analogous monotonicity properties for the relative optimal weights. We note that similarly to [19], our proofs crucially rely on the preservation of log-concavity of solutions to the heat-equation. 
Proposition 2.11. Let the relative risk tolerance coefficient $\tilde{R}(x), x>0$, be an increasing (decreasing) function. Then, for each $t \in[0, T)$ and $x>0$,

i) the optimal weights $\tilde{\pi}^{*, i}(x, t), i=1, \ldots, N$, are also increasing (decreasing).

ii) the local relative risk tolerance and risk aversion coefficients $\tilde{r}(x, t)$ and $\tilde{\gamma}(x, t)$ are, respectively, increasing (decreasing) and decreasing (increasing) functions.

Proof. Differentiating (2.31) at $t=T$ yields,

$$
\tilde{R}^{\prime}(x)=\left.\frac{\partial^{2}}{\partial x^{2}}(\log H(z, T))\right|_{z=H^{(-1)}(x, T)} .
$$

Therefore, $\tilde{R}(x)$ is an increasing function if and only if $H(x, T)$ is log-convex. Applying Proposition 2.4 for $h_{0}(x)=\tilde{R}^{\prime}(x)$ we deduce that, for each $t \in[0, T)$ and $x \geq 0$,

$$
\frac{\partial^{2}}{\partial x^{2}}(\log H(x, t))>0
$$

Using (2.31) once more, we deduce that

$$
\frac{\partial}{\partial x}(\tilde{r}(x, t))=\left.\frac{\partial^{2}}{\partial x^{2}}(\log H(z, t))\right|_{z=H^{(-1)}(x, t)},
$$

and using (2.24) we conclude.

The analogous results are readily derived when the relative risk tolerance is decreasing. In this case, one uses the preservation of the log-concavity property of the solution to the heat equation, presented in Proposition 2.5.

\subsubsection{Concavity/convexity in wealth}

We examine the spatial concavity/convexity properties of the optimal allocations and optimal weights. Whether the risk tolerance coefficient is concave or convex has been a topic of longstanding debate. We refer the reader to [55] for an extensive discussion (pp. 205-206 therein), as well as to $[83,88]$. We note, however, that there are arguments and results which support both assumptions. In particular, as proven in [63], concave risk tolerance implies that the risk aversion is proper, standard and risk vulnerable (cf., respectively, [104], [76] and [56]). The empirical study in [57] also suggests that the risk tolerance is a concave function of wealth.

Proposition 2.12. The following assertions hold:

i) Let the absolute risk tolerance coefficient $R(x), x \geq 0$, be concave. Then, for each $t \in[0, T)$ and $x \geq 0$, the optimal allocations $\pi^{*, i}(x, t), i=1, \ldots, N$ are also concave. Moreover, for each 
$t \in[0, T)$ and $x \geq 0$, the local absolute risk tolerance and risk aversion functions $r(x, t)$ and $\gamma(x, t)$ are, respectively, concave and convex.

ii) Let the absolute risk tolerance coefficient $R(x), x \geq 0$, be convex. Then, for each $t \in[0, T)$ and $x \geq 0$, the optimal allocations $\pi^{*, i}(x, t), i=1, \ldots, N$ and the local absolute risk tolerance coefficient $r(x, t)$ are also convex.

iii) Let the absolute risk aversion coefficient $A(x), x \geq 0$, be concave. Then, for each $t \in[0, T)$ and $x \geq 0$, the optimal allocations $\pi^{*, i}(x, t), i=1, \ldots, N$ are convex functions. Moreover, for each $t \in[0, T)$ and $x \geq 0$, the local absolute risk tolerance and risk aversion coefficients $r(x, t)$ and $\gamma(x, t)$ are, respectively, convex and concave.

Proof. To establish (i), we differentiate (2.31) twice at $t=T$ to obtain

$$
R^{\prime \prime}(x)=\left.\frac{\partial^{2}}{\partial x^{2}}\left(\log H_{x}(z, T)\right)\right|_{z=H^{(-1)}(x, T)} .
$$

Therefore, $R(x)$ is concave if and only if $H_{x}(x, T)$ is log-concave. In turn, we have that the function $H_{x}$ solves the heat equation (2.22) with log-concave terminal data. Applying Proposition 2.5 for $h_{0}(x)=R^{\prime \prime}(x)$,we deduce that for each $t \in[0, T)$ and $x \geq 0$, the function $H_{x}(x, t)$ is also log-concave. Differentiating (2.31) twice yields

$$
r_{x x}(x, t)=\left.\frac{\partial^{2}}{\partial x^{2}}\left(\log H_{x}(z, t)\right)\right|_{z=H^{(-1)}(x, t)},
$$

and using (2.24) we conclude. From (2.13) we have

$$
\gamma_{x x}(x, t)=-\frac{r_{x x}(x, t) r^{2}(x, t)-2\left(r_{x}(x, t)\right)^{2} r(x, t)}{r^{4}(x, t)},
$$

and the convexity of $\gamma(x, t)$ follows.

Assertion (ii) follows along similar arguments but using, instead, the preservation of the logconvexity property of solutions to the heat equation that $H_{x}(x, t)$ solves.

For (iii), we first observe that if the risk aversion coefficient $A(x)$ is a concave function, then (2.38) yields that $R(x)$ is convex and, in turn, the results of (ii) hold.

Note that the convexity of $R(x)$ (resp. $A(x)$ ) does not determine whether $A(x)$ (resp. $R(x)$ ) is concave or convex. 


\subsubsection{Monotonicity in time}

This result shows that it is exclusively the curvature of the absolute risk tolerance coefficient that determines the time monotonicity of the optimal investment function. The proof of the result is remarkably simple and just uses the fact that the local risk tolerance function solves the fast-diffusion equation (2.16).

\section{Proposition 2.13. The following assertions hold:}

Let the absolute risk tolerance coefficient $R(x), x \geq 0$, be concave (convex). Then, for each $x \geq 0$, the optimal allocations $\pi^{*, i}(x, t), i=1, \ldots, N$, are increasing (decreasing) functions of time. Moreover, the local absolute risk tolerance and risk aversion coefficients $r(x, t)$ and $\gamma(x, t)$ are, respectively, increasing and decreasing functions of time.

ii) Let the absolute risk aversion coefficient $A(x), x \geq 0$, be concave. Then, for each $x \geq 0$, the optimal allocations $\pi^{*, i}(x, t), i=1, \ldots, N$, are decreasing functions of time. Moreover, the local absolute risk tolerance and risk aversion coefficients are, respectively, decreasing and increasing functions.

Proof. We only show (i) since the rest of the arguments follow easily. To this end, recall from Proposition 2.12 that if $R(x)$ is concave (convex) then, for each $t \in[0, T)$, the absolute local risk tolerance $r(x, t)$ is also concave (convex). From equation (2.16) we, then, observe than $r_{t}(x, t) \leq 0(\geq 0)$, and the assertion follows.

For the case of concave terminal risk tolerance, the above results is quite surprising for it goes against the conventional wisdom that as investors get older they should decrease their allocations in the risky assets. We refer the reader to [108] for further discussion of this assumption, upon which, for example, so-called life-cycle funds rely. As pointed out above, there are studies supporting the use of utility functions with concave as well as convex risk tolerance functions.

The temporal behaviour of the value function and the optimal policies have been examined in more extended model settings in ${ }^{1}[27]$ and [84]. Therein, however, the generality of the model did not allow for specific results as the one above. After this thesis was completed, the author found that in [85] the case of convex risk tolerance and its effect on the time behaviour of the optimal policy was established using duality and martingale techniques.

\footnotetext{
${ }^{1}$ The author thanks the examiners of this DPhil thesis for pointing out these valuable references which, in particular, led to the discovery of Lazrak's work.
} 


\subsubsection{Robustness of the optimal allocations and optimal weights}

In this section we investigate the robustness of the optimal allocations and optimal weights with respect to ordered terminal data. Specifically, we consider two investors whose absolute risk tolerance coefficients, say $R_{1}(x)$ and $R_{2}(x)$, satisfy, for all $x \geq 0$,

$$
R_{1}(x) \leq R_{2}(x)
$$

and we examine whether the above inequality implies that, for all $(x, t) \in \mathbb{R}_{+} \times[0, T)$ and each $i=1, \ldots, N$, the optimal feedback functionals $\pi_{j}^{*, i}(x, t)$ and $\tilde{\pi}_{j}^{*, i}(x, t), j=1,2$, are, respectively, similarly ordered,

$$
\pi_{1}^{*, i}(x, t) \leq \pi_{2}^{*, i}(x, t) \quad \text { and } \quad \tilde{\pi}_{1}^{*, i}(x, t) \leq \tilde{\pi}_{2}^{*, i}(x, t)
$$

It is immediate that one needs to check whether (2.39) is preserved at all previous times, namely, if for all $(x, t) \in \mathbb{R}_{+} \times[0, T)$,

$$
r_{1}(x, t) \leq r_{2}(x, t)
$$

To our knowledge, these robustness questions have been investigated only by Xia in [125] for the log-normal model. Therein, the author considered an approximating sequence of penalized versions of the fast-diffusion equation in (2.16) and used duality arguments to obtain comparison of their solutions. As he mentions (see Remark 4.3 in [125]), it is quite difficult to obtain comparison results directly from (2.16).

However, as we show below, such comparison results can be obtained. The key idea is to consider an auxiliary equation, specifically, the one satisfied by the square of the risk tolerance function (cf. (2.53)), and establish the comparison for this equation rather than for the original one. The comparison for the fast-diffusion equation (2.16) then follows using the positivity of the local absolute risk tolerance functions.

Our proof is substantially shorter and more direct than the one in [125]. We note though that the results hold for a slightly smaller class of absolute risk tolerance coefficients. Specifically, we consider risk tolerances $R(x)$ which, for $x \geq 0$, satisfy

$$
R^{\prime}(x)<+\infty
$$

and, moreover, their square $R^{2}(x)$ is a semi-superharmonic function $(S S H)$, namely, it satisfies the inequality

$$
\left(R^{2}(x)\right)^{\prime \prime} \leq K
$$

for some $K \in \mathbb{R}$. We provide examples of such absolute risk tolerance coefficients in the sequel. 
Before we present the main robustness result, we provide the following auxiliary properties. We remind the reader that $R(x)$ is taken to be strictly increasing ( $D A R A$ hypothesis). We note however that all arguments in Propositions 2.14 and 2.16 below go through even when $R(x)$ is not monotone, at the expense of slightly more complicated arguments. However, we do not consider this case because of modeling considerations.

Proposition 2.14. Assume that the absolute risk tolerance coefficient $R(x)$ satisfies (2.42) and (2.43), for all $x \geq 0$, and some $K \in \mathbb{R}$. Then, the following assertions hold:

i) The function $r^{2}(x, t)$ is also SSH, uniformly in $t$, namely, for $(x, t) \in \mathbb{R}_{+} \times[0, T)$,

$$
\left(r^{2}(x, t)\right)_{x x} \leq K
$$

ii) The absolute risk tolerance coefficient $R(x)$ satisfies, for all $x \geq 0$, and $C=\sqrt{|K| / 2}$,

$$
R^{\prime}(x) \leq C \quad \text { and } \quad R(x) \leq C x .
$$

In turn, for $(x, t) \in \mathbb{R}_{+} \times[0, T)$,

$$
r_{x}(x, t) \leq C \quad \text { and } \quad r(x, t) \leq C x .
$$

Proof. i) To show (2.44), we first use (2.31) for $t=T$ to obtain $R^{2}(x)=H_{x}^{2}\left(H^{(-1)}(x, T), T\right)$. Therefore,

$$
\begin{gathered}
\left(R^{2}(x)\right)^{\prime}=2\left(H^{(-1)}(x, T)\right)^{\prime} H_{x}\left(H^{(-1)}((x, T), T)\right) H_{x x}\left(H^{(-1)}(x, T), T\right) \\
=2 H_{x x}\left(H^{(-1)}(x, T), T\right) .
\end{gathered}
$$

In turn,

$$
\left(R^{2}(x)\right)^{\prime \prime}=2\left(H^{(-1)}(x, T)\right)^{\prime} H_{x x x}\left(H^{(-1)}(x, T), T\right)
$$

and, thus,

$$
\left(R^{2}(x)\right)^{\prime \prime}=\left.2 \frac{H_{x x x}(z, T)}{H_{x}(z, T)}\right|_{z=H^{(-1)}(x, T)} .
$$

Using (2.24) and (2.43), we deduce that, for $x \geq 0$,

$$
H_{x x x}(x, T) \leq M H_{x}(x, T),
$$

with $M=\frac{1}{2} K$. Classical comparison results for the heat equation, which both $H_{x x x}(x, t)$ and $M H_{x}(x, t)$ satisfy, then imply that the above inequality is preserved for previous times, i.e. that

$$
H_{x x x}(x, t) \leq M H_{x}(x, t)
$$


for all $(x, t) \in \mathbb{R}_{+} \times[0, T)$. On the other hand, using (2.31) once more yields, for $t \in[0, T)$,

$$
\left(r^{2}(x, t)\right)_{x x}=\left.2 \frac{H_{x x x}(z, t)}{H_{x}(z, t)}\right|_{z=H^{(-1)}(x, t)},
$$

and (2.44) follows from (2.48).

The first part of (2.45) follows from a direct adaptation of Proposition 2.4 in [52]; for completeness we highlight the main steps. We only look at the case that $K>0$.

To this end, from (2.43) we have that the function $R^{2}(x)-\frac{1}{2} K x^{2}$ is concave. Therefore,

$$
R^{2}(y)-\frac{1}{2} K y^{2} \leq R^{2}(x)-\frac{1}{2} K x^{2}+\left(R^{2}(x)-\frac{1}{2} K x^{2}\right)^{\prime}(y-x),
$$

for any $y>0$. Taking $y=x+h, x \geq 0$, we deduce that for all $h \in \mathbb{R}$,

$$
\frac{1}{2} K h^{2}+\left(R^{2}(x)\right)^{\prime} h+R^{2}(x) \geq 0 .
$$

Therefore, we must have $\left(\left(R^{2}(x)\right)^{\prime}\right)^{2}-2 K R^{2}(x) \leq 0$.

In turn, using that $R(x)$ and $R^{\prime}(x)$ are nonnegative, we obtain that $\left(R^{2}(x)\right)^{\prime} \leq \sqrt{2 K} R(x)$, and we easily conclude.

The second part of (2.45) follows directly from the first and the fact that $R(0)=0$.

To show the first part of (2.46), we use that $R^{\prime}(x) \leq C$ (cf. (2.45)) and $R^{\prime}(x)=\left.\frac{H_{x x}(z, T)}{H_{x}(z, T)}\right|_{z=H^{(-1)}(x, T)}$ to deduce that $\frac{H_{x x}(x, T)}{H_{x}(x, T)} \leq C$. Working as above we obtain that, for all $(x, t) \in \mathbb{R}_{+} \times[0, T)$, $H_{x x}(x, t) \leq C H_{x}(x, t)$, and we easily conclude.

The second part of (2.46) follows along similar arguments.

Remark 2.15. In [125], the admissible class of risk tolerance functions satisfy $R(x) \leq M(1+x)$, for $x \geq 0$ and $M>0$. This property allows for risk tolerances with $\lim _{x \downarrow 0} R^{\prime}(x)=\infty$, which, however, are excluded herein due to (2.42). It is this property that makes our admissible class smaller than the one in [125]. Such a case is, for example, $R(x)=\sqrt{x}$. Note that this risk tolerance is SSH, for it satisfies $\left(R^{2}(x)\right)^{\prime \prime}=0$, but fails to satisfy (2.42) at $x=0$.

We are now ready to present the main robustness result.

Proposition 2.16. Assume that the absolute risk tolerance coefficients $R_{1}(x)$ and $R_{2}(x)$ satisfy, for $x \geq 0$,

$$
R_{1}(x) \leq R_{2}(x),
$$

and inequalities (2.42) and (2.43). Then, for $(x, t) \in \mathbb{R}_{+} \times[0, T)$ : 
i) the optimal feedback allocations $\pi_{j}^{*, i}(x, t)$ and optimal weights, $\tilde{\pi}_{j}^{*, i}(x, t), x>0, i=1, \ldots, N$, and $j=1,2$, satisfy

$$
\pi_{1}^{*, i}(x, t) \leq \pi_{2}^{*, i}(x, t) \quad \text { and } \quad \tilde{\pi}_{1}^{*, i}(x, t) \leq \tilde{\pi}_{2}^{*, i}(x, t),
$$

ii) the local absolute and relative risk tolerance and risk aversion functions satisfy

$$
r_{1}(x, t) \leq r_{2}(x, t) \quad \text { and } \quad \gamma_{1}(x, t) \geq \gamma_{2}(x, t)
$$

and

$$
\tilde{r}_{1}(x, t) \leq \tilde{r}_{2}(x, t) \quad \text { and } \quad \tilde{\gamma}_{1}(x, t) \geq \tilde{\gamma}_{2}(x, t) .
$$

Proof. We only show the first inequality in (2.51), since all other assertions follow trivially. We will provide the proof in a slightly more general way.

To this end, we consider two functions, say $r_{1}(x, t)$ and $r_{2}(x, t)$, with the following properties: they are $C^{2,1}\left(\mathbb{R}_{+} \times[0, T]\right)$, positive with $r_{1}(0, t)=r_{2}(0, t)=0$, and, for each $t \in[0, T)$, strictly increasing. They are, also, respectively a sub- and super-solution of (2.16) and satisfy at $t=T$ the inequality (2.50). Moreover, $r_{1}^{2}(x, t)$ and $r_{2}^{2}(x, t)$ satisfy $(2.42)$ and (2.43), and $r_{2}(x, t) \leq K x$.

We first observe that the fast-diffusion equation in (2.16) yields that the function $F: \mathbb{R}_{+} \times[0, T] \rightarrow$ $\mathbb{R}_{+}$defined by

$$
F(x, t)=r^{2}(x, t)
$$

satisfies the quasi-linear equation

$$
F_{t}+\frac{1}{2}|\lambda|^{2} F F_{x x}-\frac{1}{4}|\lambda|^{2} F_{x}^{2}=0 \quad \text { with } \quad F(x, T)=R^{2}(x) .
$$

To facilitate the exposition we will work with $\bar{F}: \mathbb{R}_{+} \times[0, T] \rightarrow \mathbb{R}_{+}$, defined by $\bar{F}(x, t)=$ $F(x, T-t)$. Then,

$$
\bar{F}_{t}-\frac{1}{2}|\lambda|^{2} \bar{F} \bar{F}_{x x}+\frac{1}{4}|\lambda|^{2} \bar{F}_{x}^{2}=0 \quad \text { with } \quad \bar{F}(x, 0)=R^{2}(x) .
$$

Therefore, the functions $f(x, t)$ and $g(x, t)$ given by

$$
f(x, t)=r_{1}^{2}(x, T-t) \quad \text { and } \quad g(x, t)=r_{2}^{2}(x, T-t)
$$

satisfy, respectively,

$$
\begin{aligned}
& f_{t}-\frac{1}{2}|\lambda|^{2} f f_{x x}+\frac{1}{4}|\lambda|^{2} f_{x}^{2} \leq 0, \\
& g_{t}-\frac{1}{2}|\lambda|^{2} g g_{x x}+\frac{1}{4}|\lambda|^{2} g_{x}^{2} \geq 0,
\end{aligned}
$$

and

$$
f(0, t)=g(0, t)=0 \quad \text { and } \quad f(x, 0) \leq g(x, 0) .
$$


Moreover, by the $S S H$ assumption, we have that for some $K \in \mathbb{R}$, for all $x \geq 0$,

$$
f_{x x}(x, 0) \leq K \text { and } g_{x x}(x, 0) \leq K .
$$

For the rest of the proof, we assume without loss of generality that $K>0$.

We are going to show that, for $t \in(0, T)$,

$$
f(x, t) \leq g(x, t)
$$

We follow part of the proof of Theorem 3.1 in [52] for comparison of positive solutions to (2.54) which are also semi-superharmonic. Because we work with smooth solutions of (2.54), various arguments in [52] are simplified, in particular the ones involving "doubling" the space variables.

To this end, we first introduce the auxiliary functions

$$
f_{m}(x, t)=\left(1-\frac{t}{m}\right) f(x, t) \quad \text { and } \quad g_{m}(x, t)=\left(1-\frac{t}{m}\right) g(x, t),
$$

where

$$
m=\min \left(T, m_{0}\right)
$$

where the constant $m_{0}$ will be chosen in the sequel, see (2.67).

Then, for $(x, t) \in \mathbb{R}_{+} \times[0, m]$,

$$
0 \leq f_{m}(x, t) \leq f(x, t) \quad \text { and } \quad 0 \leq g_{m}(x, t) \leq g(x, t) .
$$

Moreover,

$$
f_{m}(x, 0) \leq g_{m}(x, 0) \quad \text { and } \quad f_{m}(x, m)=g_{m}(x, m)=0,
$$

and

$$
f_{m}(0, t)=g_{m}(0, t)=0 .
$$

We then easily obtain that $f_{m}$ and $g_{m}$ satisfy, respectively,

$$
\left(1-\frac{t}{m}\right) f_{m, t}+\frac{1}{m} f_{m}-\frac{1}{2}|\lambda|^{2} f_{m} f_{m, x x}+\frac{1}{4}|\lambda|^{2} f_{m, x}^{2} \leq 0
$$

and

$$
\left(1-\frac{t}{m}\right) g_{m, t}+\frac{1}{m} g_{m}-\frac{1}{2}|\lambda|^{2} g_{m} g_{m, x x}+\frac{1}{4}|\lambda|^{2} g_{m, x}^{2} \geq 0 .
$$

As argued in [52], straightforward bootstrapping argumentation can be used to establish (2.55) for $(x, t) \in \mathbb{R}_{+} \times(0, T]$ once it is shown that, for $(x, t) \in \mathbb{R}_{+} \times[0, m]$,

$$
f_{m}(x, t) \leq g_{m}(x, t)
$$


To this end, we consider the test function $\varphi(x)=1+x^{4}$, for $x \geq 0$. We are going to show that, for any $\varepsilon>0$, we have

$$
f_{m}(x, t) \leq g_{m}(x, t)+\varepsilon \varphi(x) .
$$

We argue by contradiction, i.e. we assume that there exists $\varepsilon>0$ such that

$$
\sup _{(x, t) \in \mathbb{R}_{+} \times[0, m]}\left(f_{m}(x, t)-g_{m}(x, t)-\varepsilon \varphi(x)\right)>0 .
$$

Let $(\hat{x}, \hat{t})$ be any point such that $f_{m}(\hat{x}, \hat{t})-g_{m}(\hat{x}, \hat{t})-\varepsilon \varphi(\hat{x})>0$. We easily get from $(2.57)$ and (2.46) that $h(\hat{x}, \hat{t}) \leq C \hat{x}^{2}$, for $h=f_{m}, g_{m}$, which together with the growth of $\varphi(x)$ yields that $\hat{x}<\infty$.

Next, we observe that the extremum in $(2.62)$, denoted by $(\tilde{x}, \tilde{t})$ is an interior point in $(0, \infty) \times$ $(0, m)$. Indeed, if $(\tilde{x}, \tilde{t})$ is such that $\tilde{t}=0$ or $\tilde{t}=m$ we get a contradiction from (2.58), while if, for some $\tilde{t} \in(0, m),(\tilde{x}, \tilde{t})=(0, \tilde{t})$, we contradict $(2.59)$.

At the interior maximum $(\tilde{x}, \tilde{t})$ in $(2.62)$ we, then, have

$$
\begin{gathered}
f_{m}(\tilde{x}, \tilde{t})-g_{m}(\tilde{x}, \tilde{t})>\varepsilon\left(1+\tilde{x}^{4}\right) \\
f_{m, t}(\tilde{x}, \tilde{t})-g_{m, t}(\tilde{x}, \tilde{t})=0, \quad f_{m, x}(\tilde{x}, \tilde{t})-g_{m, x}(\tilde{x}, \tilde{t})=4 \varepsilon \tilde{x}^{3}
\end{gathered}
$$

and

$$
f_{m, x x}(\tilde{x}, \tilde{t})-g_{m, x x}(\tilde{x}, \tilde{t}) \leq 12 \varepsilon \tilde{x}^{2}
$$

From (2.60) and (2.61) we deduce

$$
\begin{gathered}
\frac{1}{m}\left(f_{m}(\tilde{x}, \tilde{t})-g_{m}(\tilde{x}, \tilde{t})\right) \leq \frac{1}{2}|\lambda|^{2}\left(f_{m}(\tilde{x}, \tilde{t}) f_{m, x x}(\tilde{x}, \tilde{t})-g_{m}(\tilde{x}, \tilde{t}) g_{m, x x}(\tilde{x}, \tilde{t})\right) \\
-\frac{1}{4}|\lambda|^{2}\left(f_{m, x}^{2}(\tilde{x}, \tilde{t})-g_{m, x}^{2}(\tilde{x}, \tilde{t})\right) .
\end{gathered}
$$

In turn,

$$
\begin{gathered}
\left(\frac{1}{m}-\frac{1}{2}|\lambda|^{2} f_{m, x x}\right)\left(f_{m}(\tilde{x}, \tilde{t})-g_{m}(\tilde{x}, \tilde{t})\right) \\
\leq \frac{1}{2}|\lambda|^{2} g_{m}(\tilde{x}, \tilde{t})\left(f_{m, x x}(\tilde{x}, \tilde{t})-g_{m, x x}(\tilde{x}, \tilde{t})\right) \\
-\frac{1}{4}|\lambda|^{2}\left(f_{m, x}(\tilde{x}, \tilde{t})-g_{m, x}(\tilde{x}, \tilde{t})\right)\left(f_{m, x}(\tilde{x}, \tilde{t})+g_{m, x}(\tilde{x}, \tilde{t})\right) .
\end{gathered}
$$

Because $f_{m}(\tilde{x}, \tilde{t})-g_{m}(\tilde{x}, \tilde{t})>0$ (cf. (2.63)), the (2.43) property of $f_{m}$ and (2.64) and (2.65) above yield

$$
\begin{gathered}
\left(\frac{1}{m}-\frac{1}{2}|\lambda|^{2} K\right)\left(f_{m}(\tilde{x}, \tilde{t})-g_{m}(\tilde{x}, \tilde{t})\right) \\
\leq 6 \varepsilon|\lambda|^{2} g_{m}(\tilde{x}, \tilde{t}) \tilde{x}^{2}-\varepsilon|\lambda|^{2} \tilde{x}^{3}\left(f_{m, x}(\tilde{x}, \tilde{t})+g_{m, x}(\tilde{x}, \tilde{t})\right) .
\end{gathered}
$$


Using that the functions $r_{1}(x, t)$ and $r_{2}(x, t)$, and in turn $f_{m}(x, t)$ and $g_{m}(x, t)$, are strictly increasing, the above inequality yields that at $(\tilde{x}, \tilde{t})$ we must have,

$$
\varepsilon\left(\frac{1}{m}-\frac{1}{2}|\lambda|^{2} K\right)\left(1+\tilde{x}^{4}\right) \leq 6 \varepsilon|\lambda|^{2} g_{m}(\tilde{x}, \tilde{t}) \tilde{x}^{2},
$$

Finally, using that $g_{m}(\tilde{x}, \tilde{t}) \leq\left(1-\frac{\tilde{t}}{m}\right) \frac{K}{2} \tilde{x}^{2} \leq \frac{K}{2} \tilde{x}^{2}$, the above inequality gives $\frac{1}{m}-\frac{1}{2}|\lambda|^{2} K \leq$ $3|\lambda|^{2} K$ and, in turn, that

$$
\frac{1}{m} \leq \frac{7}{2}|\lambda|^{2} K
$$

We easily see that if we choose $m_{0}$ in (2.56) such that

$$
\min \left(T, m_{0}\right)<\frac{2}{7|\lambda|^{2} K},
$$

we get a contradiction, and we easily conclude.

Next, we provide examples of utility functions whose absolute risk tolerance coefficient satisfies (2.42) and (2.43).

Example 2.17. The popular power case, $U(x)=\frac{1}{\gamma} x^{\gamma}, \gamma \in(0,1)$, has $R^{2}(x)=\left(\frac{1}{1-\gamma}\right)^{2} x^{2}$, satisfies (2.43) (as equality) with $K=2\left(\frac{1}{1-\gamma}\right)^{2}$.

Example 2.18. Consider a utility function whose inverse marginal $I(x)$ (cf. (2.5)) is of the form

$$
I(x)=\int_{0}^{N} x^{-y} \nu(d y),
$$

for some finite and positive Borel measure (utility functions of this type are further discussed in Section 2.2 below), and with compact support $(N<\infty)$. Then, (2.23) yields that the related harmonic function is given, at $t=T$, by $H(x, T)=\int_{0}^{N} e^{x y} \nu(d y)$. From (2.35) we deduce that, for $x \geq 0$,

$$
R^{\prime}(x)=2 \frac{\int_{0}^{N} y^{2} e^{H^{(-1)}(x, T) y} \nu(d y)}{\int_{0}^{N} y e^{H^{(-1)}(x, T) y} \nu(d y)} \leq 2 N
$$

and (2.42) follows. Moreover, (2.43) holds since

$$
\left(R^{2}(x)\right)^{\prime \prime}=2 \frac{\int_{0}^{N} y^{3} e^{H^{(-1)}(x, T) y} \nu(d y)}{\int_{0}^{N} y e^{H^{(-1)}(x, T) y} \nu(d y)} \leq 2 N^{2} .
$$

We finish this section with a corollary of the above results which yields time-independent upper and lower bounds for the optimal policies. These bounds are linear functions of wealth. As a result, we are able to relate policies under general risk preferences with the ones of power utility, say $U(x)=\frac{1}{\delta} x^{\delta}$, with the parameter $\delta$ depending only on the slope of the risk tolerance coefficient at $x=0$, as described in (2.69) below. We remind the reader that $R^{\prime}(0)<\infty$ (cf. (2.42)). 
Corollary 2.19. Let the absolute risk tolerance coefficient $R(x)$ be such that $R^{\prime}(0)>0$ and define $\delta<1$ as

$$
\delta=1-\frac{1}{R^{\prime}(0)} .
$$

Then, for all $t \in[0, T]$, the optimal allocations $\pi^{*, i}(x, t), i=1, \ldots, N$, satisfy:

If $R(x)$ is a concave function,

$$
\pi^{*, i}(x, t) \leq \frac{1}{1-\delta} x \sigma^{-1} \lambda
$$

while, if $R(x)$ is a convex function,

$$
\pi^{*, i}(x, t) \geq \frac{1}{1-\delta} x \sigma^{-1} \lambda
$$

Proof. We only show (2.70). To this end, we first note that because $R(x)$ is concave and $R(0)=0$, we have that $R(x) \leq R^{\prime}(0) x$. Trivially, the function $R^{\prime}(0) x$ satisfies (2.43) with $K=2 R^{\prime}(0)$. Using the comparison result in Proposition 2.16, we deduce that for $(x, t) \in$ $\mathbb{R}_{+} \times[0, T), r(x, t) \leq R^{\prime}(0) x$. In turn, (2.10) yields that $\pi^{*, i}(x, t) \leq R^{\prime}(0) x \sigma^{-1} \lambda, i=1, \ldots, N$, and we conclude.

\subsubsection{Sensitivity analysis}

\subsubsection{Sensitivities with respect to market parameters}

We study the dependence on the market coefficients of the local absolute risk tolerance and risk aversion functions $r(x, t)$ and $\gamma(x, t)$, as well as of the optimal feedback portfolios.

For reasons we discuss later on, we start with the case in which only one stock is traded. We then denote by $\sigma$ and $\lambda$, respectively, its volatility coefficient and Sharpe ratio (cf. (2.2)), and assume that $\lambda, \sigma>0$. We note that even in this simple case, it is not immediate how the market parameters affect $r(x, t), \gamma(x, t)$ and $\pi^{*}(x, t)$, for $\lambda$ and $\sigma$ appear in a convoluted way. For example, while the terminal condition $r(x, T)$ is independent on $\lambda$, for $t \in[0, T)$, we have that $r(x, t ; \lambda)=-\frac{u_{x}(x, t ; \lambda)}{u_{x x}(x, t ; \lambda)}$, with both $u_{x}(x, t ; \lambda)$ and $u_{x x}(x, t ; \lambda)$ depending on $\lambda$, as it can be seen from the HJB equation (2.8). To our knowledge, sensitivity analysis for these functions has not been carried out to date for general utility functions.

For the case of a single stock, the equations that $r(x, t)$ and $\pi^{*}(x, t)$ solve (cf. (2.16) and (2.18)), simplify to

$$
r_{t}+\frac{1}{2} \lambda^{2} r^{2} r_{x x}=0 \quad \text { and } \quad \pi_{t}^{*}+\frac{1}{2} \sigma^{2}\left(\pi^{*}\right)^{2} \pi_{x x}^{*}=0
$$

with

$$
r(x, T)=-\frac{U^{\prime}(x)}{U^{\prime \prime}(x)} \quad \text { and } \quad \pi^{*}(x, T)=-\frac{\lambda}{\sigma} \frac{U^{\prime}(x)}{U^{\prime \prime}(x)}
$$


We start with the monotonicity of the local absolute risk tolerance and risk aversion functions with respect to $\lambda$ and $\sigma$. As we show below, their dependence on the market parameters depends exclusively on the curvature of the absolute risk tolerance coefficient $R(x)$. This result is quite surprising and in contradistinction with the monotonicity of the optimal investment policy $\pi^{*}(x, t)$ which always preserves its corresponding monotonicity properties, independently on the concavity/convexity of $R(x)$.

A standing assumption for the next two propositions is that inequalities (2.42) and (2.43) is satisfied and, therefore, the comparison results in Proposition 2.16 hold.

Proposition 2.20. i) Let the absolute risk tolerance coefficient $R(x), x \geq 0$, be concave. Then, for $(x, t) \in \mathbb{R}_{+} \times[0, T)$, the local absolute risk tolerance $r(x, t)$ is decreasing in $\lambda$ and increasing in $\sigma$. The reverse assertion holds for the local absolute risk aversion $\gamma(x, t)$.

ii) Let the absolute risk tolerance coefficient $R(x), x \geq 0$, be convex. Then, for $(x, t) \in \mathbb{R}_{+} \times$ $[0, T)$, the local risk tolerance function $r(x, t)$ is increasing in $\lambda$ and decreasing in $\sigma$. The reverse assertion holds for the local absolute risk aversion $\gamma(x, t)$.

ii) Let the absolute risk aversion coefficient $A(x), x \geq 0$, be concave. Then, for $(x, t) \in \mathbb{R}_{+} \times$ $[0, T)$, the local absolute risk aversion function $\gamma(x, t)$ is increasing in $\lambda$ and decreasing in $\sigma$. The reverse assertion holds for the local risk tolerance function $r(x, t)$.

Proof. We only show the first part of assertion (i) since the rest of the statements follow trivially. To this end, let $\lambda>\hat{\lambda}$, and denote by $r(x, t ; \lambda)$ and $r(x, t ; \hat{\lambda})$ the corresponding solutions to (2.72) and (2.73). We are going to show that, for $(x, t) \in \mathbb{R}_{+} \times[0, T]$,

$$
r(x, t ; \lambda) \leq r(x, t ; \hat{\lambda}) .
$$

As shown in Proposition 2.12, if $R(x)$ is concave, the local absolute risk tolerance function $r(x, t)$ is also concave, for each $t \in[0, T)$. Therefore,

$$
\begin{gathered}
r_{t}(x, t ; \hat{\lambda})+\frac{1}{2} \lambda^{2} r^{2}(x, t ; \hat{\lambda}) r_{x x}(x, t ; \hat{\lambda}) \\
=r_{t}(x, t ; \hat{\lambda})+\frac{1}{2} \hat{\lambda}^{2} r^{2}(x, t ; \hat{\lambda}) r_{x x}(x, t ; \hat{\lambda})+\frac{1}{2}\left(\lambda^{2}-\hat{\lambda}^{2}\right) r^{2}(x, t ; \hat{\lambda}) r_{x x}(x, t ; \hat{\lambda}) \leq 0,
\end{gathered}
$$

where we used that $r(x, t ; \hat{\lambda})$ solves $(2.16)$ and $r_{x x}(x, t ; \hat{\lambda}) \leq 0$. Therefore, $r(x, t ; \hat{\lambda})$ is a supersolution to the equation that $r(x, t ; \lambda)$ solves with $r(x, T ; \lambda)=r(x, T ; \hat{\lambda})$. Using the comparison result in Proposition 2.16, (2.74) follows.

The next result examines the monotonicity of the optimal feedback portfolio $\pi^{*}(x, t)$ in terms of $\lambda$ and $\sigma$. We show that independently of the convexity/concavity of the absolute risk tolerance 
coefficient, and thus of the analogous properties of the terminal condition $\pi^{*}(x, T)$, the optimal portfolio function is always increasing in $\lambda$ and decreasing in $\sigma$.

Proposition 2.21. The optimal feedback portfolio function $\pi^{*}(x, t)$ is always increasing in $\lambda$ and decreasing in $\sigma$.

Proof. Let $\lambda>\hat{\lambda}$ and denote by $\pi^{*}(x, t ; \lambda)$ and $\pi^{*}(x, t ; \hat{\lambda})$ the corresponding portfolio functions. Then, for fixed $\sigma$, both functions solve equation (2.72) which is independent of the Sharpe ratio, while $\pi^{*}(x, T ; \lambda) \geq \pi^{*}(x, T ; \hat{\lambda})$. Using the robustness result of Proposition 2.16 we easily conclude.

The monotonicity with respect to $\sigma$ is slightly more involved since $\sigma$ appears in both the equation and the terminal condition. To this end, we define the auxiliary function $\bar{\pi}(x, t ; \sigma)=\sigma \pi^{*}(x, t ; \sigma)$, which solves

$$
\bar{\pi}_{t}+\frac{1}{2} \bar{\pi}^{2} \bar{\pi}_{x x}=0 \quad \text { and } \quad \bar{\pi}(x, T)=\lambda R(x) .
$$

Therefore, $\bar{\pi}(x, t ; \sigma)$ is independent of $\sigma$, yielding $\frac{\partial \bar{\pi}(x, t ; \sigma)}{\partial \sigma}=0$. In turn, we deduce that $\frac{\partial \pi^{*}(x, t ; \sigma)}{\partial \sigma}=$ $-\frac{1}{\sigma} \pi^{*}(x, t ; \sigma)$ and using the positivity of $\pi^{*}(x, t ; \sigma)$ we conclude.

For the investment model with more than one stock analogous results can be obtained even though the intuitive interpretation of the market parameter $m_{i}$ below is limited.

Proposition 2.22. i) Let the absolute risk tolerance coefficient $R(x)$ be concave (convex). Then, for $(x, t) \in \mathbb{R}_{+} \times[0, T)$, the local absolute risk tolerance $r(x, t)$ is decreasing (increasing) in $|\lambda|^{2}$.

ii) Let the absolute risk aversion coefficient $A(x)$ be concave. Then, for $(x, t) \in \mathbb{R}_{+} \times[0, T)$, the local risk aversion $\gamma(x, t)$ is increasing in $|\lambda|^{2}$.

From (2.18) and (2.19) we have that each optimal feedback portfolio component $\pi^{*, i}(x, t)$ satisfies

$$
\pi_{t}^{*, i}+\frac{1}{2} m_{i}^{2}\left(\pi^{*, i}\right)^{2} \pi_{x x}^{*, i}=0 \quad \text { and } \quad \pi^{*, i}(x, T)=\frac{|\lambda|}{m_{i}} R(x) .
$$

We then have the following result.

Proposition 2.23. For each $i=1, \ldots, N$, the optimal feedback component $\pi^{*, i}(x, t)$ is increasing in $|\lambda|$ and decreasing in $m_{i}$, where $m_{i}=k_{i}^{-1}|\lambda|$ with $k_{i}$ being the $i^{\text {th }}$ element of the vector $\sigma^{-1} \lambda$. 


\subsubsection{Sensitivities of the optimal processes in terms of the initial wealth}

We conclude with the sensitivities of the optimal wealth and portfolio processes with respect to the initial condition. They follow from direct differentiation in the stochastic representations (2.32) and (2.33). To our knowledge, with the exception of (2.75) which was derived for a much more general market setting in [81], assertions (2.76), (2.77) and (2.78) are new.

Proposition 2.24. The following assertions hold:

i) Let $X_{t}^{*}, t \in[0, T]$, be the optimal wealth process with $X_{0}^{*}=x$. Then,

$$
\frac{\partial X_{t}^{*}}{\partial x}=\frac{r\left(X_{t}^{*}, t\right)}{r(x, 0)}
$$

and

$$
\frac{\partial^{2} X_{t}^{*}}{\partial x^{2}}=\frac{r_{x}\left(X_{t}^{*}, t\right)-r_{x}(x, 0)}{r(x, 0)} \frac{\partial X_{t}^{*}}{\partial x}=\frac{r\left(X_{t}^{*}, t\right)}{r^{2}(x, 0)}\left(r_{x}\left(X_{t}^{*}, t\right)-r_{x}(x, 0)\right) .
$$

ii) Let $\pi_{t}^{*}, t \in[0, T]$, be the optimal portfolio process. Then,

$$
\frac{\partial \pi_{t}^{*}}{\partial x}=\frac{\partial X_{t}^{*}}{\partial x} r_{x}\left(X_{t}^{*}, t\right) \sigma^{-1} \lambda=\frac{r\left(X_{t}^{*}, t\right)}{r(x, 0)} r_{x}\left(X_{t}^{*}, t\right) \sigma^{-1} \lambda
$$

and

$$
\begin{gathered}
\frac{\partial^{2} \pi_{t}^{*}}{\partial x^{2}}=\left(\frac{\partial^{2} X_{t}^{*}}{\partial x^{2}} r_{x}\left(X_{t}^{*}, t\right)+\left(\frac{\partial X_{t}^{*}}{\partial x}\right)^{2} r_{x x}\left(X_{t}^{*}, t\right)\right) \sigma^{-1} \lambda \\
=\frac{r\left(X_{t}^{*}, t\right)}{r^{2}(x, 0)}\left(r_{x}\left(X_{t}^{*}, t\right)\left(r_{x}\left(X_{t}^{*}, t\right)-r_{x}(x, 0)\right)+r\left(X_{t}^{*}, t\right) r_{x x}\left(X_{t}^{*}, t\right)\right) \sigma^{-1} \lambda .
\end{gathered}
$$

Proof. To show (2.75) we differentiate (2.32) with respect to the initial condition to get

$$
\frac{\partial X_{t}^{*}}{\partial x}=\frac{H_{x}\left(H^{(-1)}(x, 0)+|\lambda|^{2} t+|\lambda| \cdot W_{t}, t\right)}{H_{x}\left(H^{(-1)}(x, 0), 0\right)} .
$$

On the other hand,

$$
\begin{gathered}
H_{x}\left(H^{(-1)}(x, 0)+|\lambda|^{2} t+|\lambda| \cdot W_{t}, t\right) \\
=H_{x}\left(H^{(-1)}\left(H\left(H^{(-1)}(x, 0)+|\lambda|^{2} t+|\lambda| \cdot W_{t}, t\right), t\right), t\right)=H_{x}\left(H^{(-1)}\left(X_{t}^{*}, t\right), t\right),
\end{gathered}
$$

where we used (2.32) once more. Using (2.31), we have that $r\left(X_{t}^{*}, t\right)=H_{x}\left(H^{(-1)}\left(X_{t}^{*}, t\right), t\right)$ and we conclude.

Differentiating (2.75) with respect to the initial condition in turn yields

$$
\frac{\partial^{2} X_{t}^{*}}{\partial x^{2}}=\frac{\partial X_{t}^{*}}{\partial x} \frac{r_{x}\left(X_{t}^{*}, t\right)}{r(x, 0)}-\frac{r_{x}(x, 0)}{r^{2}(x, 0)} r\left(X_{t}^{*}, t\right)
$$




$$
=\frac{r\left(X_{t}^{*}, t\right)}{r(x, 0)} \frac{r_{x}\left(X_{t}^{*}, t\right)}{r(x, 0)}-\frac{r_{x}(x, 0)}{r^{2}(x, 0)} r\left(X_{t}^{*}, t\right)=\frac{r\left(X_{t}^{*}, t\right)}{r^{2}(x, 0)}\left(r_{x}\left(X_{t}^{*}, t\right)-r_{x}(x, 0)\right) .
$$

To show (2.77) we use that $\pi_{t}^{*}=r\left(X_{t}^{*}, t\right) \sigma^{-1} \lambda$ and (2.75). Equation (2.78) follows along similar arguments.

Using the stochastic representations (2.32) and (2.9), we can express the above sensitivities in terms of the spatial derivatives of the underlying harmonic function $H(x, t)$.

Corollary 2.25. Let $H(x, t)$ be the solution of (2.22) and (2.23) and $N_{t}=H^{(-1)}(x, 0)+|\lambda|^{2} t+$ $|\lambda| \cdot W_{t}$. Then,

$$
\frac{\partial X_{t}^{*}}{\partial x}=\frac{H_{x}\left(N_{t}, t\right)}{H_{x}\left(N_{0}, 0\right)} \quad \text { and } \quad \frac{\partial^{2} X_{t}^{*}}{\partial x^{2}}=\frac{H_{x}\left(N_{t}, t\right)}{H_{x}^{2}\left(N_{0}, 0\right)}\left(\frac{H_{x x}\left(N_{t}, t\right)}{H_{x}\left(N_{t}, t\right)}-\frac{H_{x x}\left(N_{0}, 0\right)}{H_{x}\left(N_{0}, 0\right)}\right),
$$

as well as

$$
\frac{\partial \pi_{t}^{*}}{\partial x}=\frac{H_{x x}\left(N_{t}, t\right)}{H_{x}\left(N_{0}, 0\right)} \sigma^{-1} \lambda,
$$

and

$$
\begin{gathered}
\frac{\partial^{2} \pi_{t}^{*}}{\partial x^{2}}=\frac{H_{x}\left(N_{t}, t\right)}{H_{x}^{2}\left(N_{0}, 0\right)} \\
\times\left(\frac{H_{x x}\left(N_{t}, t\right)}{H_{x}\left(N_{t}, t\right)}\left(\frac{H_{x x}\left(N_{t}, t\right)}{H_{x}\left(N_{t}, t\right)}-\frac{H_{x x}\left(N_{0}, 0\right)}{H_{x}\left(N_{0}, 0\right)}\right)+\frac{H_{x x x}\left(N_{t}, t\right) H_{x}\left(N_{t}, t\right)-H_{x x}^{2}\left(N_{t}, t\right)}{H_{x}^{2}\left(N_{t}, t\right)}\right) \sigma^{-1} \lambda .
\end{gathered}
$$

\subsection{Structural representations of utilities and their effects on horizon flexibility and stochastic dominance}

In this section we study two different classes of utility functions. Specifically, utility functions for which the marginal utility and the inverse marginal utility, respectively, are so-called completely monotonic functions (see precise statement below). We show that these structural properties give rise to complementary properties. While the use of the former class of utilities implies that the (standard Merton) expected utility criterion respects stochastic dominance of any order, the use of the latter class yields that the investment-problem can be extended (in a consistent way) to arbitrary horizons. The main conclusions are summarized in Section 2.2.4.

Throughout this section, we consider the log-normal market model and the Merton investment problem specified in Section 2.1.1. We note that some result in this section hold also under slightly weaker assumptions on the utility function $U(x)$ but do not pursue this issue any further. Moreover, we let $N=1$ but note that the results readily extends also to the case of multiple risk assets. For notational reasons we redefine as follows (cf. (2.21)):

$$
h(y):=H(y, T)=I\left(e^{-y}\right) .
$$


Note that, up to affine transformations, a utility function $U$ is uniquely defined via either of the functions $I$ or $h$. Since the utility maximization problem is equivalent up to affine transformations of the utility function, we therefore identify a utility function with its corresponding functions $I$ and $h$, respectively, and without further notice we use either of them to refer to a specific utility function.

\subsubsection{Complete monotonicity and two specific classes of utility func- tions}

The aim is to study two different classes of utility functions, both closely related to completely monotonic functions, and compare the different features of the corresponding preferences. To this end, we first recall the definition of complete monotonicity. Then, in turn, the respective classes of utility functions are defined.

Definition 2.26 (Absolutely Monotonic Functions). A function $f(x)$ is absolutely monotonic $(A M)$ in the interval $a<x<b$ if it has non-negative derivatives of all orders, namely,

$$
f^{(n)}(x) \geq 0 \quad n=0,1,2, \ldots .
$$

A function is completely monotonic (CM) in the interval $a<x<b$ if and only if $f(-x)$ is absolutely monotonic in the interval $-b<x<-a$.

First, we consider the class of so called 'completely monotone utilities', which are frequently considered in the literature; see among others [22], [104] and the references therein. The notion was introduced by Brocket and Golden in [22]. We recall their definition.

Definition 2.27 (Brocket and Golden). An admissible utility function (cf. page 16) is said to be a completely monotone utility if $U^{\prime}(x)$ is a completely monotonic function.

Next, we introduce the second class of utility functions. To this end we define the following set of positive Borel measures.

$$
\mathcal{B}:=\left\{\mu \text { defined on } \mathcal{B}\left(\mathbb{R}_{+}\right): \forall B \in \mathcal{B}\left(\mathbb{R}_{+}\right), \mu(B) \geq 0 \quad \text { and } \quad \int_{0}^{\infty} e^{x y} \mu(d y)<+\infty, \quad x \in \mathbb{R}\right\} .
$$

Definition 2.28. For each measure $\mu \in \mathcal{B}$, the associated function $h^{\mu}: \mathbb{R} \rightarrow \mathbb{R}_{+}$is defined by

$$
h^{\mu}(y)=\int_{0}^{\infty} e^{y s} \mu(d s), \quad-\infty<y<\infty,
$$

and the associated utility function (cf. (2.79)) is denoted by $U^{\mu}(x)$. 
We note that this class of preferences has appeared previously (see, among others, [96] and further discussion in Section 3.1.4.2). As established next, also these utility functions are closely related to the notion of complete monotonicity.

Proposition 2.29. The following are equivalent:

i) It holds that $U(x)=U^{\mu}(x)$ for some measure $\mu \in \mathcal{B}$,

ii) the associated function $h(y): \mathbb{R} \rightarrow \mathbb{R}_{+}$is an absolutely monotonic function on $y \in \mathbb{R}$,

iii) the associated function $I(y): \mathbb{R}_{+} \rightarrow \mathbb{R}_{+}$is given by

$$
I(y)=\int_{0}^{\infty} y^{-s} \mu(d s) .
$$

In particular, for the above conditions to hold true, it is a necessary condition that $I(y)$ is a completely monotonic function on $y \in \mathbb{R}_{+}$.

Proof. According to Definition 2.28, $U(x)=U^{\mu}(x)$, for some $\mu \in \mathcal{B}$, if and only if the associated function $h$ is given by (2.81) for some $\mu \in \mathcal{B}$. Hence, statements i) and ii) are equivalent according to Corollary 2.39 given in Section 2.2.6 below.

Next, note that the functions $I: \mathbb{R}_{+} \rightarrow \mathbb{R}_{+}$and $h: \mathbb{R} \rightarrow \mathbb{R}_{+}$(cf. (2.79)) are related via

$$
I(y)=h(-\ln y), \quad y>0 .
$$

It holds that the function $f: \mathbb{R}_{+} \rightarrow \mathbb{R}$ defined via $f(x)=-\ln x$ satisfies $(-1)^{k} f^{(k)}(x) \geq 0$ for $k=1, \ldots$. Hence, if ii) holds and $h(y)$ is AM, then $I(y)$ is the composition of an AM and a CM function and, thus, itself CM (cf. Theorem IV 2.b in [120]). Specifically, combining (2.82) with the representation of $h$ given in (2.80) yields

$$
I(y)=\int_{0}^{\infty} e^{s(-\ln y)} \mu(d s)=\int_{0}^{\infty} y^{-s} \mu(d s),
$$

and formula (2.81) follows. Hence, i) implies iii). Conversely, it also follows for any function $I$, which is representable by (2.81) for some measure $\mu$, that the associated function $h(y)$ is given by (2.80). Hence, iii) implies i) and we conclude.

We note that for $I: \mathbb{R}_{+} \rightarrow \mathbb{R}_{+}$to be represented as in (2.81), it is a necessary but not a sufficient condition that $I$ is completely monotonic. To illustrate this, let $I$ be a CM. According to Bernstein's theorem (cf. Theorem 2.38 below), it is, thus, represented by

$$
I(y)=\int_{0}^{\infty} e^{-s y} d \alpha(s), \quad y \geq 0
$$


for some measure $\alpha$. According to the above result, it suffices to show that there exists some $\alpha$ such that the associated function $h$, given by

$$
h(y)=I\left(e^{-y}\right)=\int_{0}^{\infty} e^{-s e^{-y}} d \alpha(s)
$$

does not satisfy the condition of absolute monotonicity. To this end, it suffices to consider $I(y)=e^{-y}$, for which $h(y)=e^{-e^{-y}}$, since a direct calculation ${ }^{2}$ yields that it is not absolutely monotonic. This should come as no surprise since $h$ (cf. (2.84)) is a composition of two CM functions ( $I$ and $f(x)=e^{-x}$ ) and the composition of two CM function, in general, is not AM (nor CM, cf. page 145 in [120]). We note though that for $I(y)=e^{-y}, \lim _{y \rightarrow 0} I(y)=1$. Hence, it does not correspond to an admissible utility function, as it does not satisfy the Inada conditions (cf. (2.4)) given by

$$
\lim _{y \rightarrow \infty} I(y)=0 \quad \text { and } \quad \lim _{y \rightarrow 0} I(y)=\infty .
$$

However, although more involved, examples of completely monotonic functions $I$ which cannot be represented as in (2.81) but do satisfy the Inada condition (2.85) can also be constructed.

While completely monotone utilities are characterized by the fact that their marginals are completely monotonic functions, utility functions $U^{\mu}, \mu \in \mathcal{B}$, are, thus, characterized by the property that the inverse of the marginal is a completely monotonic function of the specific type defined in (2.81). As established next, these structural properties, respectively, give rise to very specific features.

\subsubsection{Preservation of stochastic dominance - complete monotonicity of the marginals}

The notion of completely monotone utilities is closely linked to the concept of stochastic dominance. In order to specify this, we define as follows:

$$
\mathcal{A}_{k}:=\left\{U: \mathbb{R}_{+} \rightarrow \mathbb{R}:(-1)^{i} U^{(i+1)}(x) \geq 0, x>0, i=1,2, \ldots, k\right\} .
$$

Then, for two random variables $X$ and $Y, X$ is said to stochastically dominate $Y$ in the $k$-th order if $\mathrm{E}[U(X)] \geq \mathrm{E}[U(Y)]$ for all functions $U \in \mathcal{A}_{k}$ (provided that the expressions are well-defined). Note that stochastic dominance is a form of stochastic ordering which compares distributions rather than random variables and that stochastic dominance of the $k$-th order implies stochastic dominance of all higher orders $k+1, k+2, \ldots$.

\footnotetext{
${ }^{2}$ Indeed, the first three derivatives are given by:$$
\left\{\begin{array}{l}
g^{\prime}(y)=e^{-\left(e^{-y}+y\right)} \\
g^{\prime \prime}(y)=e^{-\left(e^{-y}+y\right)}\left(e^{-y}-1\right) \\
g^{\prime \prime \prime}(y)=e^{-\left(e^{-y}+y\right)}\left(e^{-2 y}-3 e^{-y}+1\right)
\end{array}\right.
$$

and, thus, it is not AM. 
A standard utility maximizer, whose utility function is increasing and concave, therefore always prefers outcome $X$ to $Y$ if $X$ stochastically dominates $Y$ in the second (or first) order. As stochastic dominance is a central and natural concept within decision theory, classes of utility functions respecting also higher order stochastic dominance have been extensively studied in the economics literature. The completely monotone utilities (utility functions with completely monotone marginals) are central within this context as they belong to $\mathcal{A}_{k}$ for all $k$. Hence, an investor with a completely monotone utility respects stochastic dominance with respect to any order. This motivated the study of these utilities in, among others, [22]. Note also that many of the standard utility functions such as logarithmic, power and exponential utilities belong to this class.

As for utility functions of the type defined in Definition 2.28 (with completely monotonic inverse marginals), the next result establishes some properties on the preservation of stochastic dominance and its relative risk aversion.

Theorem 2.30. Let $U^{\mu}(x), \mu \in \mathcal{B}$, and denote by $R^{\mu}: \mathbb{R}_{+} \rightarrow \mathbb{R}_{++}$and $\tilde{R}^{\mu}: \mathbb{R}_{+} \rightarrow \mathbb{R}_{++}$, the associated absolute and relative risk tolerance, respectively (cf. (2.11) and (2.12)). Then, the following holds:

i) In addition to being increasing and concave, $U^{\mu}(x)$ also satisfies $U_{x x x}^{\mu}(x) \geq 0, x \in \mathbb{R}_{+}$. Hence, it respects stochastic dominance up to 3rd order (prudence).

ii) Moreover, $R^{\mu}(x)$ is strictly increasing and convex in wealth and, furthermore, $\tilde{R}^{\mu}(x)$ is increasing in wealth.

Note that preservation of higher order stochastic dominance is neither guaranteed nor usually satisfied for these utility functions.

Proof. According to Proposition 2.29, it holds that $I(y)=\left(U^{\prime}\right)^{-1}$ is CM and, hence, statement i) is a direct consequence of Lemma 2.45 below. Next, in order to prove ii), note that according to Definition 2.28, the function $h: \mathbb{R} \rightarrow \mathbb{R}_{+}$associated with $U^{\mu}$ via (2.79), is given by (2.80). Hence, $h \in C^{\infty}$ and, specifically, the $k^{\text {th }}$ derivative of $h$ is given by

$$
\frac{\partial^{k}}{\partial y^{k}} h(y)=\int_{0}^{\infty} s^{k} e^{y s} \mu(d s) .
$$

In particular, $h_{y y}(y)>0$ and, thus, according to $(2.35)$, it follows that $R^{\mu}(x)$ is strictly increasing in $x$. Next, define the matrices $A(y)$ and $C(y)$ via

$$
A(y):=\left[\begin{array}{cc}
h_{y}(y) & h_{y y}(y) \\
h_{y y}(y) & h_{y y y}(y)
\end{array}\right], \quad C(y):=\left[\begin{array}{cc}
h(y) & h_{y}(y) \\
h_{y}(y) & h_{y y}(y)
\end{array}\right] .
$$


By use of (2.86), it follows that for any non-zero vector, say $\xi=\left[\begin{array}{ll}\xi_{0} & \xi_{1}\end{array}\right]$, with $\xi_{0}, \xi_{1} \in \mathbb{R}$, that

$$
\begin{aligned}
\xi A(y) \xi^{T}=\sum_{i, j=0}^{1} \frac{\partial^{1+i+j}}{\partial y^{1+i+j}} h(y) \xi_{i} \xi_{j} & =\int_{0}^{\infty} \sum_{i, j=0}^{1} s^{1+i+j} \xi_{i} \xi_{j} e^{y s} \mu(d s) \\
& =\int_{0}^{\infty} s\left(\sum_{i=0}^{1} s^{i} \xi_{i}\right)^{2} e^{y s} \mu(d s) \geq 0 .
\end{aligned}
$$

Consequently, $A(y), y \in \mathbb{R}$, is positive semi-definite and, thus, according to Sylvester's criterion (cf. [53]) $h_{y}(y)$ is log-convex. Then, in turn, it follows from $(2.37)$ that $R^{\mu}(x)$ is convex in $x$. A similar argument yields

$$
\xi C(y) \xi^{T}=\sum_{i, j=0}^{1} \frac{\partial^{i+j}}{\partial y^{i+j}} h(y) \xi_{i} \xi_{j} \geq 0 .
$$

Hence, $C(y), y \in \mathbb{R}$, is also positive semi-definite. Using once again Sylvester's criterion yields $h(y)$ log-convex which, in turn, implies that $\tilde{R}^{\mu}(x)$ is increasing in $x$ (cf. (2.36)).

\subsubsection{Horizon flexibility - complete monotonicity of the inverse marginals}

In this section, we study several horizon aspects of the portfolio choice problem. In particular, we show that utility functions of the type $U^{\mu}, \mu \in \mathcal{B}$, (with completely monotone inverses as defined in (2.81)) admit a certain type of horizon flexibility (cf. Theorem 2.32 below).

Consider an investor with a fixed terminal horizon $T$ and a utility function $U$ set at that date. The investor then starts to invest according to the associated optimal strategy. However, at some point she decides to extend trading beyond the initial horizon $T$, up to, say $\bar{T}>T$. Then, the question we want to address is:

- can the investment problem, once formulated, be extended until time $\bar{T}>T$ in a way which is consistent with the original problem?

Next, we formalize the notion of an extended investment problem.

Definition 2.31. For a given horizon $T>0$ and utility function $U(x)$, the associated portfolio choice problem can be extended until $\bar{T}>T$, if there exists an admissible utility function $\bar{U}(x)$ such that, set at $\bar{T}$, its associated value function $\bar{u}: \mathbb{R}_{+} \times[0, \bar{T}] \rightarrow \mathbb{R}$, defined in (2.7), satisfies

$$
\bar{u}(x, T ; \bar{T})=U(x) . \quad \forall x \in \mathbb{R}_{+} .
$$

Note that if $(2.87)$ holds, then $\bar{u}(x, t ; \bar{T})=u(x, t ; T)$ for all $t \leq T$, where the latter value function is associated with the utility function $U(x)$. 
Theorem 2.32. Consider a fixed terminal date $T$ at which the investor has risk preferences given by the utility function $U(x)$. Then, the following statements are equivalent:

i) The investment problem can be extended until time $\bar{T}$, for arbitrary $\bar{T}>T$,

ii) There exists a measure $\mu \in \mathcal{B}$ such that

$$
U(x)=U^{\mu}(x)
$$

Proof. To start with, we claim that i) holds if and only if there exists a function $u: \mathbb{R}_{+} \times[0, \infty) \rightarrow$ $\mathbb{R}$, which solves

$$
\left\{\begin{array}{l}
u_{t}-\frac{\lambda^{2}}{2} \frac{u_{x}^{2}}{u_{x x}}=0, \quad t \geq 0, x \in \mathbb{R}_{+}, \\
u(x, 0)=U(x),
\end{array}\right.
$$

and, in its spatial variable, is strictly concave, increasing and satisfies the Inada conditions (cf. (2.4)). Indeed, assume that such a solution $u(x, t), t \geq 0$, exists. Then, for any $\bar{T}>T$, the function $\bar{U}(x):=u(x, \bar{T}-T)$ is an admissible utility function such that its corresponding value function, trivially, satisfies (2.87). Hence, the problem can be extended to any $\bar{T}>T$ and i) holds.

Conversely, assume that the problem can be extended till $\bar{T}>T$. Then, the thereto related value function satisfies (2.89) for $(x, t) \in \mathbb{R}_{+} \times[0, \bar{T}-T)$ (cf. Lemma 2.3) and is strictly increasing, concave and satisfies the Inada conditions in its spatial variable. Hence, if i) holds, then there exists a well-defined solution with the required spatial properties on $\mathbb{R}_{+} \times[0, \bar{T}-T)$, for any $\bar{T}>0$. Note that the solution to (2.89) is unique on any interval $[0, \bar{T}-T)$ where it is welldefined. In consequence, a solution exists for $(x, t) \in \mathbb{R}_{+} \times[0, \infty)$. This completes the proof of the claim.

Next, for a given $u: \mathbb{R}_{+} \times[0, \infty) \rightarrow \mathbb{R}$, define the function $h: \mathbb{R} \times[0, \infty) \rightarrow \mathbb{R}_{+}$via the transformation

$$
u_{x}\left(h\left(y, t \lambda^{2} / 2\right), t\right)=e^{-y+\frac{\lambda^{2}}{2} t},
$$

and note that every given function $h: \mathbb{R} \times[0, \infty) \rightarrow \mathbb{R}_{+}$uniquely determines $u(x, t)$ up to affine transformations. Then, it can be shown (cf. Lemma 2.3; see also Proposition 15 in [96]) that $u(x, t)$ is a solution to $(2.89)$, such that $u(\cdot, t)$ is strictly concave, increasing and satisfying the Inada conditions, if and only if the associated function $h(y, t)$ is a positive solution to

$$
\left\{\begin{array}{l}
h_{t}+h_{x x}=0, \\
h(y, 0)=h(y),
\end{array}\right.
$$

such that $h(\cdot, t)$ is strictly increasing and satisfies

$$
\lim _{y \rightarrow-\infty} h(y, t)=0 \quad \text { and } \quad \lim _{y \rightarrow+\infty} h(y, t)=\infty, \quad t \geq 0 .
$$


According to Corollary 2.42 below, every solution to (2.91) which is positive and increasing in its spatial variable, must be given by

$$
h(y, t)=\int_{\mathbb{R}} e^{y s-s^{2} t} \mu(d s),
$$

for some measure $\mu \in \mathcal{B}$. Specifically, such a solution exists if and only if $h(y)=\int_{0}^{\infty} e^{y s} d \mu(s)$, $\mu \in \mathcal{B}$. That is to say, if and only if ii) holds. Since every solution given by (2.93) satisfies (2.92), we thus conclude.

The investor who invests according to a utility function of the form $U^{\mu}, \mu \in \mathcal{B}$, is, thus, not restricted to the interval $[0, T)$ but might continue, for all future times, to invest in a way which is consistent with her previous actions. Hence, these utility functions constitute a quite specific subset of utility functions (for further remarks on this class of utility functions and their appearance in the literature, see below). Given that the corresponding investment problem can be extended to arbitrary horizons in a dynamically consistent way, these utility functions should also be contrasted to the criteria considered in [119] (see, also, [10]). Indeed, therein utility functions which are not everywhere differentiable are considered. In particular, the investment problem set with respect to such a utility function can not be extended to any later horizon. Hence, these two categories of utility functions represent two extremes when it comes to extensibility.

Naturally, this raises the question of whether, and if so how far, the investment problem set with respect to a general utility functions can be extended in a dynamically consistent way. In order to address this question we give the following definition.

Definition 2.33. Given an entire function ${ }^{3} f(\cdot)$ such that $f(x) \in \mathbb{R}$ for $x \in \mathbb{R}$, define

$$
e^{-t \mathcal{D}^{2}} f(x):=\frac{1}{\sqrt{4 \pi t}} \int_{-\infty}^{\infty} e^{-y^{2} / 4 t} f(x+i y) d y, \quad x \in \mathbb{R}
$$

whenever the expression on the right hand side exists.

Next, we formulate necessary and sufficient conditions for an investment problem to be extensible to a future horizon.

Theorem 2.34. Consider a fixed terminal date $T$ and a utility function $U(x)$ with the associated function $h(y)$ (cf. (2.79)). Let $\bar{T}>T$ be given and define $\tau:=\frac{\lambda^{2}}{2}(\bar{T}-T)$. Then, the following statements are equivalent:

i) The investment problem can be extended until time $\bar{T}$ in the sense of Definition 2.31 .

\footnotetext{
${ }^{3} \mathrm{An}$ entire function is a complex-valued function which is holomorphic (complex differentiable in a neighbourhood of every point in its domain) over the whole complex plane. This class of functions coincide with the class of functions that are analytic on the whole complex plane, that is to say, functions that are equal to their Taylor-series in a neighbourhood of each point in the complex plane; see [17].
} 
ii) It holds that

- $h(x)$ is entire,

- $h(x+i y)=\mathcal{O}\left(e^{y^{2} / 4 \tau}\right)$ as $y \rightarrow{ }_{-}^{+} \infty$, uniformly in $-R \leq x \leq R$, for every $R>0$,

- $e^{-t \mathcal{D}^{2}} h(x) \geq 0$, for $0<t<\tau, x \in \mathbb{R}$,

and, furthermore, the following limit exists,

$$
\bar{h}:=\lim _{t \nearrow \tau} e^{-t \mathcal{D}^{2}} h(x)
$$

is in $C^{3}$ and satisfies

$$
\lim _{x \rightarrow-\infty} \bar{h}(x)=0, \quad \text { and } \quad \lim _{x \rightarrow+\infty} \bar{h}(x)=\infty
$$

In particular, if the above statements hold true, then the unique utility function $\bar{U}$ for which Defintion 2.31 holds, is defined via its associated function $\bar{h}$ given in (2.95).

Before proving the above result, we make some remarks. First, clearly, $h^{\mu}, \mu \in \mathcal{B}$, satisfies the above conditions for any $\tau>0$. Second, note that the above conditions are joint conditions on the utility function $U(x)$ and $\tau$, where the latter depends on $\lambda$ as well as $\bar{T}$. In particular, the presence of the Sharpe ratio $\lambda$, implies that:

- the future horizon, to which an investment problem can be extended, depends on the market as well as the utility function $U(x)$ set at the original terminal date.

Third, the above result implies that extending the investment problem, in a log-normal model, effectively corresponds to solving the heat equation backwards; a problem well-known to be illposed in the sense of Hadamard. The restrictive condition in part ii) above are, thus, imposed in order to ensure existence of a solution on the required interval. Indeed, in general the solution to the backward heat equation might explode even on finite intervals. Whenever a solution exists, it is however unique. Although the above conditions ensure existence (and uniqueness), the solution to the backward heat equation might still not depend continuously on data, which makes a numerical treatment hard.

Proof. Let $\Delta=\bar{T}-T$. By use of arguments similar to the ones used in the proof of Theorem 2.32 , it follows that $\mathrm{i}$ ) holds if and only if there exists a function $u: \mathbb{R}_{+} \times[0, \Delta] \rightarrow \mathbb{R}$ which has the following properties: it is in class $C^{4,1}\left(\mathbb{R}_{+} \times[0, \Delta]\right)$; it is strictly concave, increasing and satisfies the Inada conditions (cf. (2.4)) in its spatial variable; and it satisfies equation (2.89) for $(x, t) \in \mathbb{R}_{+} \times[0, \Delta)$.

Next, for any function $u: \mathbb{R}_{+} \times[0, \Delta] \rightarrow \mathbb{R}$, define the associated function $h: \mathbb{R} \times[0, \tau] \rightarrow \mathbb{R}$ via (2.90) and note that $h(y, t)$ (up to affine transformations) uniquely determines $u(x, t)$. Then, in 
turn, using again the same arguments as in the proof of Theorem 2.32, it follows that a function $u(x, t)$ satisfies the above properties if and only if, the associated function $h: \mathbb{R} \times[0, \tau] \rightarrow \mathbb{R}$ is in $C^{3,1}$ and a positive solution to

$$
\left\{\begin{array}{l}
h_{t}+h_{x x}=0, \\
h(y, 0)=h(y),
\end{array}\right.
$$

such that $h(\cdot, t)$ is strictly increasing and satisfies $(2.96)$ for $t \in[0, \tau]$.

Next, we claim that the existence of such a function $h(y, t)$, is equivalent to the existence of a positive, continuous and strictly increasing function $\bar{h}: \mathbb{R} \rightarrow \mathbb{R}_{+}$which satisfies (2.96) and the condition

$$
h(y)=\int_{-\infty}^{\infty} k(y-s, \tau) \bar{h}(s) d s, \quad y \in \mathbb{R},
$$

with $k(x, t)=e^{-x^{2} / 4 t} / \sqrt{4 \pi t}$. Indeed, given such an $\bar{h}(x)$, let $h: \mathbb{R} \times[0, \tau] \rightarrow \mathbb{R}_{+}$, be given by

$$
h(y, t):=\int_{-\infty}^{\infty} k(y-s, \tau-t) \bar{h}(s) d s, \quad t<\tau,
$$

and $h(x, \tau)=\bar{h}(y)$. Then, $h(y, t)$ is well-defined, positive, strictly increasing in $y$ and satisfies (2.96). Moreover, we have that $\lim _{t \nearrow_{\tau}} h(y, t)=\bar{h}(y)$ and that $h(y, t)$ solves equation (2.97). Conversely, assume there exists a positive function $h(y, t)$, which is strictly increasing in $y$ and solves (2.97). Then, let $\bar{h}(y)=h(y, \tau)$, which satisfies (2.98) by construction.

\subsubsection{The role of complete monotonicity in portfolio choice}

The aim in this section is to illustrate properties of two specific classes of utility functions, both closely related to complete monotonicity. For utility functions of the form $U^{\mu}, \mu \in \mathcal{B}$, the inverse of the marginal utility is a completely monotonic function (of a specific type), while for completely monotone utilities, the marginal utility itself is a completely monotonic function. As the next example shows, power utility plays a pivotal role in this context as it does, in fact, belong to both classes.

Example 2.35. The power utility function

$$
U(x)=\frac{1}{1-r} x^{1-r}, \quad r>0,
$$

is of type (2.88) as well as a completely monotone utility. Indeed, $U^{\prime}(x)=x^{-r}$ is a completely monotonic function and its inverse $U^{\prime(-1)}(y)=I(y)=y^{-1 / r}$ is given by (2.81) for $\mu=\delta_{1 / r}$.

The power utility is, however, the exception that proves the rule. Indeed, as established in Lemma 2.45 below, the inverse of a function given by (2.81) for some measure $\mu \in \mathcal{B}$, is in 
general not a completely monotonic function. In consequence, few utility functions belong to both classes. This relates to the fact that these two classes are characterized by two entirely different features. While completely monotone utilities respect stochastic dominance of any order, utility functions of the form (2.88) can be extended to arbitrary future horizons (but only respect up till 3rd order stochastic dominance). Rather than advertising any of these classes to be the better choice, the aim here is to emphasize the implications of certain features in the shape of the utility function.

We continue this comparison by noting that complete monotonicity of the form (2.81) of the inverse marginal utility, is a property which is preserved by the value function (at all times). This might be verified by calculation but is also an immediate consequence of Theorem 2.32. Indeed, consider $U^{\mu}, \mu \in \mathcal{B}$, and let $u(x ; t, T)$ be the associated value function. Since the investment problem associated with $U^{\mu}$ can be extended to arbitrary future horizons, it follows that so must the investment problem set with respect to $u(\cdot ; t, T)$ at the horizon $t$. According to Theorem 2.32, for each $t \leq T$, there thus exists $\tilde{\mu} \in \mathcal{B}$ such that

$$
u(x, t ; T)=U^{\tilde{\mu}}(x) .
$$

Hence, the value function associated with a utility function as defined in Definition 2.28 belongs, itself, to the same class of utility functions.

This should be contrasted to the property of complete monotonicity of the marginal utility which, in general, is not inherited by the value function. That is to say, the value function associated with a completely monotone utility need not, itself, belong to the class of completely monotone utilities. That the value function (at least in some neighbourhood around $T$ ) preserves key properties yields a certain horizon flexibility. Indeed, consider an investor who starts to invest according to the utility function $U(x)$ set at the terminal horizon $T$. If the value function, even for times close to $T$, does not possess the properties required from a utility function, then the investor is very exposed to the a priori choice of investment horizon. However, if there exists some neighbourhood around $T$, for which the value function itself is a feasible utility function, some flexibility with respect to the horizon is obtained.

We will return to the notion of complete monotonicity in Section 3.1.4.2 below. Next, we conclude this section with some results on an inverse investment problem and the auxiliary results used in the above proofs. These results are presented, respectively, in Sections 2.2.5 and 2.2.6.

\subsubsection{Investment policies and the inverse investment problem}

Consider the initial investment choice $\pi_{0}(x), x \in \mathbb{R}_{+}$. The aim is to study the inverse problem of deducing whether this function is optimal with respect to some utility function and, if so, 
to infer the latter. The reason to study this question is that, from a practical point of view, it is difficult for an investor to specify her utility function. Nevertheless, investors do invest. Consequently, it is of interest to see whether one from the investor's initial investment choice, can infer the utility function in accordance with which she is operating. Questions of this type has been addressed in, among others, [59] and [28] (where the latter considers the infinite horizon consumption problem).

First, we note that due to Proposition 2.7 (cf. (2.33)), the initial profile $\pi_{0}$ is directly related to the function $h(y, 0), y \in \mathbb{R}$. Hence, we define as follows.

Definition 2.36. Let the initial investment choice $\pi_{0}: \mathbb{R}_{+} \rightarrow \mathbb{R}_{+}$be given. Then, the associated inferred function $h_{0}^{\pi}: \mathbb{R} \rightarrow \mathbb{R}_{+}$is defined via

$$
h_{0}^{\pi(-1)}(x)=\frac{\lambda}{\sigma} \int_{1}^{x} \frac{d s}{\pi_{0}(s)}, \quad x>0,
$$

where $h_{0}^{\pi(-1)}(x)$ denotes its spatial inverse.

The following corollary then follows from Theorem 2.34 and Lemma 2.40 below. It explores the information about risk preferences incorporated in the initial investment choice.

Corollary 2.37. Let an initial investment choice $\pi_{0}(x), x \in \mathbb{R}_{+}$be given. Let $h_{0}^{\pi}$ be defined as in Definition 2.36 and consider the horizon $T>0$. Then the following holds,

i) The initial investment $\pi_{0}(x)$ is optimal with respect to some utility function $U(x)$ set at $T$, if and only if $h_{0}^{\pi}$ satisfies the conditions in ii) of Theorem 2.34.

In particular, if such a utility function $U(x)$ exists, then it is uniquely defined via its associated function $h$ given in (2.112).

ii) The initial investment $\pi_{0}(x)$ is optimal with respect to a utility function given by $u^{\mu}(x)$ set at $T$, for some $\mu \in \mathcal{B}$, if and only if the function $h_{0}^{\pi}$ is absolutely monotonic in $\mathbb{R}$.

In particular, if such a utility function $u^{\mu}$ exists and, furthermore, the associated measure $\mu$ admits the representation $d \mu(s)=\alpha(s) d s$ for some function $\alpha$, then the function $\alpha$ : $\mathbb{R}_{+} \rightarrow \mathbb{R}_{+}$is given by

$$
\alpha(s)=e^{-s^{2} \lambda^{2} T / 2} \mathcal{L}^{-1}\left\{h_{0}^{\pi}(-s), s>0\right\},
$$

where $\mathcal{L}^{-1}$ denotes the inverse-Laplace operator. 
For the particular case when $d \mu(s)=\alpha(s) d s$, the above result yields that the measure $\mu$ is uniquely determined by $h_{0}^{\pi}(y)$, for $y<0$. In fact, this can be shown to hold true for each measure $\mu \in \mathcal{B}$. Hence, since $h_{0}^{\pi}:(-\infty, 0] \rightarrow(0,1]$ is the inverse of the function $h_{0}^{\pi(-1)}:(0,1] \rightarrow(-\infty, 0]$ which, in turn, is given by (2.99) for $0<x<1$, it follows that the knowledge of $\pi_{0}(x), 0<x<1$, suffices to determine the measure $\mu$. Note, however, that absolute monotonicity of $h_{0}^{\pi}$ on all of $\mathbb{R}$ is needed in order to ensure that $\pi_{0}$ actually is an optimal policy which corresponds to a forward criterion.

The main point of the above result is that while the utility function is an abstract object that is hard to specify, the investors' initial investment choice might be obtained from an investor. Theoretically, one could therefore identify the function $\pi_{0}(x)$ and, then, in turn, via Corollary 2.37 check if that investment choice is in accordance with some utility function. If so, the latter could even be recovered. Note, however, that even when the above conditions are satisfied, there are associated computational issues. Indeed, to recover the utility function from the initial investment choice effectively amounts to solve the heat equation backwards; a problem wellknown to be ill-posed in the sense of Hadamard. Hence, even if we have existence (which is ensured by the conditions in iii) of Theorem 2.34) and uniqueness (which always holds for the backward heat equation) the solution might not depend continuously on the data which makes a numerical treatment hard.

From a theoretical point of view, it is still interesting to be able to infer the terminal utility function solely from the initial investment choice. Specifically, the information of the investor's optimal investments at a single point in time, is thus enough to reveal her optimal investment strategy at all times and, in particular, whether or not she considers a utility of the form (2.88).

\subsubsection{Auxiliary results on completely monotonic functions}

In this section, some well-known and useful results will be recalled. Moreover, certain corollaries which have been made us of in this chapter, are established and proven.

\section{Bernstein's theorem}

Theorem 2.38 (Bernstein: Thm IV 12 in [120]). A function $f$ is $C M$ in $(0, \infty)$ if and only if

$$
f(x)=\int_{0}^{\infty} e^{-s x} d \alpha(s), \quad 0<x<\infty,
$$

for some positive measure $\alpha$ such that the integral converges for $0<x<\infty$. 
Corollary 2.39. $\quad$ i) A function $f$ is $C M$ in $(-\infty, \infty)$ if and only if

$$
f(x)=\int_{0}^{\infty} e^{-s x} d \alpha(s), \quad-\infty<x<\infty,
$$

for some positive measure $\alpha$ such that the integral converges for $-\infty<x<\infty$.

ii) A function $f$ is $A M$ in $(-\infty, \infty)$ if and only if

$$
f(x)=\int_{0}^{\infty} e^{s x} d \alpha(s), \quad-\infty<x<\infty
$$

for some positive measure $\alpha$ such that the integral converges for $-\infty<x<\infty$.

Proof. Let $\eta>0$. Since $f(x)$ is CM for $-\infty<x<\infty$, it holds, trivially, that $f(x)$ is CM for $x>-\eta$. Define the function $g(x)$ via $g(x):=f(x-\eta), x>0$. It follows that $g(x)$ is CM for $x>0$. Thus, according to Theorem 2.38 , there exists a measure $\mu^{\eta}$ such that

$$
g(x)=\int_{0}^{\infty} e^{-s x} d \mu^{\eta}(s), \quad x>0,
$$

where the integral converges for $x>0$. Since $f(x)=g(x+\eta), x>-\eta$, this, in turn, implies that

$$
\begin{aligned}
f(x) & =\int_{0}^{\infty} e^{-s(x+\eta)} d \mu^{\eta}(s) \\
& =\int_{0}^{\infty} e^{-s x} d \alpha^{\eta}(s), \quad x>-\eta,
\end{aligned}
$$

where the integral converges for $x>-\eta$ and where

$$
\alpha^{\eta}(t)=\int_{0}^{t} e^{-\eta s} d \mu^{\eta}(s) .
$$

Next, since $f(x)$ is CM for $-\infty<x<\infty$, it is in particular CM for $x>0$. Hence, we might also use Theorem 2.38 to obtain,

$$
f(x)=\int_{0}^{\infty} e^{-s x} d \alpha(s), \quad x>0,
$$

for some measure $\alpha$ and where the integral converges for $x>0$. However, the Laplace integral representation of a function defined on $x>0$ is unique (Theorem 6.3 in [120], p 63). Hence, comparison of formulae (2.101) and (2.102) (where both integrals converge for $x>0$ ) yields that

$$
\alpha^{\eta}(s)=\alpha(s), \quad s>0 .
$$

Hence, it follows from (2.101) that

$$
f(x)=\int_{0}^{\infty} e^{-s x} d \alpha(s), \quad x>-\eta,
$$


where the integral converges for $x>-\eta$. Since $\eta$ was chosen arbitrarily, the representation in (2.103) holds for arbitrary $\eta>0$. Hence, it holds for $-\infty<x<\infty$ and the result in part i) follows.

Next, if $f(x)$ is AM for $-\infty<x<\infty$, then it holds that $g(x):=f(-x)$ is CM on $-\infty<x<\infty$. Hence, it follows from part i) that

$$
g(x)=\int_{0}^{\infty} e^{-s x} d \alpha(s), \quad-\infty<x<\infty,
$$

for some measure $\alpha$. Part ii) follows since $f(x)=g(-x)$.

We also have the following result.

Lemma 2.40. Let $f^{1}(x)$ and $f^{2}(x)$ be CM in $-\infty<x<\infty$. Then, it holds that if $f^{1}(x)=f^{2}(x)$ for $x \in[a, b], a, b \in \mathbb{R}$, then $f^{1}(x)=f^{2}(x)$ for $-\infty<x<\infty$.

Proof. A function which is completely monotonic on $\mathbb{R}$ is analytic on $\mathbb{R}$ and, therefore, it can be extended to an analytic function in the entire plane (analytic continuation). Since an analytic function is uniquely determined by its values along a curve in its area of analyticity, the result follows.

\section{Widder's theorem}

Theorem 2.41 (Widder: p 235 in [123]). All positive solutions, $h: \mathbb{R} \times[0,+\infty) \rightarrow \mathbb{R}_{+}$, to the equation

$$
h_{t}+h_{x x}=0, \quad x \in \mathbb{R}, \quad t>0 .
$$

are given by

$$
h(x, t)=\int_{\mathbb{R}} e^{x y-y^{2} t} \mu(d y),
$$

for some measure $\mu \in \mathcal{B}$.

Corollary 2.42. Let $h: \mathbb{R} \times[0,+\infty) \rightarrow \mathbb{R}_{+}$, and consider equation (2.104). Then, the following statements are equivalent:

i) $h(x, t)$ is a solution to (2.104), and $h(x, t)$ and $h_{x}(x, t)$ are positive,

ii) $h(x, t)$ is a solution to (2.104) and absolutely monotonic on $\mathbb{R}$ in its spatial argument,

iii) $h(x, t)$ is given by (2.105) for a measure $\mu$ in $\mathcal{B}$ such that $\mu((-\infty, 0])=0$. 
Proof. It follows from Theorem 2.41, that every positive solution $h: \mathbb{R} \times[0,+\infty) \rightarrow \mathbb{R}_{+}$to equation (2.104) is given by

$$
h(x, t)=\int_{-\infty}^{\infty} e^{x y-y^{2} t} \mu(d y)
$$

for some positive measure $\mu$. Next, calculate the spatial derivative of this expression to obtain

$$
h_{x}(x, t)=\int_{-\infty}^{\infty} e^{x y-y^{2} t} y \mu(d y) .
$$

Moreover, note that the function $h_{x}$ also solves equation (2.104). Indeed, by differentiating (2.104) with respect to $x$ we obtain $\left(h_{x}\right)_{t}+\frac{\lambda^{2}}{2}\left(h_{x}\right)_{x x}=0, x \in \mathbb{R}$ and $t>0$. This implies that if $h_{x}$ is a positive function we can apply Theorem 2.41 to this function as well to obtain the representation

$$
h_{x}(x, t)=\int_{\mathbb{R}} e^{x y-y^{2} t} \nu(d y),
$$

for some positive measure. Now, for (2.108) and (2.107) to be equal for all $x$ in $\mathbb{R}$, we need the measure $\mu$ to be of positive support, as $\mu$ and $\nu$ are both positive measures. Consequently, i) implies iii).

Next, calculate the spatial derivatives of $h(x, t)$, given by (2.106) for some $\mu \in \mathcal{B}$ of positive support, to obtain

$$
h^{(n)}(x, t)=\int_{\mathbb{R}_{+}}(y)^{n} e^{x y-y^{2} t} \mu(d y)>0, \quad x \in \mathbb{R}, \quad n=1,2, \ldots
$$

Thus, $h(x, 0)$ is absolutely monotonic in its spatial variable on the entire real line. Moreover, $h^{(n)}(x, t)<+\infty, n=1,2, \ldots$, since it follows from Hölder's inequality that

$$
\left|h^{(n)}(x, t)\right| \leq \int_{\mathbb{R}_{+}}\left|(y)^{n} e^{x y-y^{2} t}\right| \mu(d y) \leq\left[\int_{\mathbb{R}_{+}} e^{2 x y-y^{2} 2 t} \mu(d y)\right]^{1 / 2}\left[\int_{\mathbb{R}_{+}} e^{2 n y} \mu(d y)\right]^{1 / 2}<+\infty,
$$

where the last inequality follows as $\mu \in \mathcal{B}$, which implies that $\int_{\mathbb{R}} e^{x y-y^{2} t} \mu(d y)<+\infty$, for all $x \in \mathbb{R}$ and $t>0$. Consequently, iii) implies ii). Trivially, ii) implies i) and we conclude.

\section{Inversion of the Weierstrass-transform}

Theorem 2.43 (Widder: Theorem 3 in [121]). For a function $f(\cdot)$ to be represented in the form

$$
f(x)=c \int_{-\infty}^{\infty} e^{-(x-y)^{2} / 4 \tau} d \alpha(y), \quad x \in \mathbb{R}, \quad c=(4 \pi \tau)^{-1 / 2},
$$

where $\alpha(y)$ is a Borel measure such that $\alpha(B) \geq 0, B \in \mathcal{B}(\mathbb{R})$, it is necessary and sufficient that:

i) $f(x)$ is entire,

ii) $f(x+i y)=\mathcal{O}\left(e^{y^{2} / 4 \tau}\right)$ as $y \rightarrow \pm_{-}^{+} \infty$, uniformly in $-R \leq x \leq R$, for every $R>0$, 
iii) $e^{-t \mathcal{D}^{2}} f(x) \geq 0$, for $0<t<\tau, x \in \mathbb{R}$.

In [121], the above result is stated for $\tau \equiv 1$, for which the transformation (2.110) corresponds to the so called Weierstrass-transform. A closer look at the proof therein, reveals, however, that the argument holds for arbitrary $\tau>0$. Next, we establish a slightly modified version of the above result.

Corollary 2.44. Let the operator $e^{-t \mathcal{D}^{2}}, 0<t<\tau$, be as in Definition 2.33 and let $\phi: \mathbb{R} \rightarrow \mathbb{R}_{+}$ be a continuous function. Consider a function $f: \mathbb{R} \rightarrow \mathbb{R}_{+}$. Then,

$$
f(x)=W_{\tau}[\phi(x)]:=c \int_{-\infty}^{\infty} e^{-(x-y)^{2} / 4 \tau} \phi(y) d y, \quad x \in \mathbb{R},
$$

if and only if $f(x)$ satisfies conditions i) - iii) of Theorem 2.43 and, furthermore,

$$
\lim _{t \nearrow \tau} e^{-t \mathcal{D}^{2}} f(x)=\phi(x) .
$$

Proof. We first show the necessary part. Let $f$ be given by (2.111), which is a particular case of (2.110). Hence, it follows from Theorem 2.43 that conditions i) to iii) of that theorem hold. In particular, $f(x)$ is entire and, moreover, $f(x+i y)=\mathcal{O}\left(e^{y^{2} / 4 \tau}\right)$ as $y \rightarrow \pm \infty$, this uniformly in $-R \leq x \leq R$ for every $R>0$. Thus, $e^{-t \mathcal{D}^{2}} f(x)$, as defined in (2.94), converges for $x \in \mathbb{R}$ and $0<t<\tau$. Next, we substitute for $f(x)$ as given, by assumption, in (2.111). It is shown in [121] (p. 435) that one thereby obtains

$$
e^{-t \mathcal{D}^{2}} f(x)=\frac{c}{(\tau-t)^{1 / 2}} \int_{-\infty}^{\infty} e^{-(x-y)^{2} / 4(\tau-t)} \phi(y) d y, \quad 0<t<\tau .
$$

It remains to show that the right hand side converges to $\phi$ as $t \nearrow \tau$. According to Theorem 5 in [122] (p. 67), this would follow if we can show that $\phi$ is Lebesgue integrable in every finite interval and satisfies

$$
\int_{a}^{x} \phi(y)-\phi(a) d y=o(|x-a|), \text { as } x \rightarrow a .
$$

However, as $\phi$ is continuous, it is Lebesgue integrable in every finite interval. Moreover, for any $\varepsilon>0$, there exists $\delta$ such that, for all $x$ with $|x-a|<\delta$, it holds that $|\phi(x)-\phi(a)|<\varepsilon$, which, in turn, implies that

$$
\int_{a}^{x}|\phi(y)-\phi(a)| d y<\varepsilon|x-a| .
$$

Therefore, $\lim _{x \rightarrow a} \int_{a}^{x}|\phi(y)-\phi(a)| d y /|x-a|=0$, which is equivalent to the condition of (2.113) and, thus, $e^{-t \mathcal{D}^{2}} f(x)$ converges to $\phi$ as $t \nearrow \tau$.

To show the sufficient part, let $f$ satisfy conditions i) - iii) of Theorem 2.43 and (2.112). According to Theorem 2.43, $f$ is given by (2.110) for some Borel measure $\alpha$ such that $\alpha(B) \geq 0, B \in \mathcal{B}(\mathbb{R})$. By the same arguments as used above, $e^{-t \mathcal{D}^{2}} f(x)$, as defined in (2.94), converges for $x \in \mathbb{R}$ and 
$0<t<\tau$. Moreover, it holds by assumption that $\lim _{t \tau_{\tau}} e^{-t \mathcal{D}^{2}} f(x)=\phi(x)$. Consequently, it follows that $f$ is given by (2.111), if it can be verified that

$$
W_{\tau}\left[\lim _{t \nearrow \tau} e^{-t \mathcal{D}^{2}} f(x)\right]=f(x),
$$

whenever $f$ is given by (2.110) for some measure $\alpha$ as specified above. It remains to show that this is indeed the case. To this end, consider $e^{-t \mathcal{D}^{2}} f(x)$ and substitute for $f(x)$, as given in (2.110). As argued above (cf. p 435 in [121]) one thereby obtains

$$
e^{-t \mathcal{D}^{2}} f(x)=\frac{c}{(\tau-t)^{1 / 2}} \int_{-\infty}^{\infty} e^{-(x-y)^{2} / 4(\tau-t)} d \alpha(y), \quad 0<t<\tau .
$$

Hence, according to Definition 2.33 we obtain

$$
\begin{aligned}
W_{\tau}\left[\lim _{t{ }_{\tau}} e^{-t \mathcal{D}^{2}} f(x)\right] & =c \int_{-\infty}^{\infty} e^{-(x-y)^{2} / 4 \tau} \lim _{t \nearrow \tau} \frac{c}{(\tau-t)^{1 / 2}} \int_{-\infty}^{\infty} e^{-(y-s)^{2} / 4(\tau-t)} d \alpha(s) d y \\
& =c \int_{-\infty}^{\infty} \lim _{t \nearrow \tau} \frac{c}{(\tau-t)^{1 / 2}} \int_{-\infty}^{\infty} e^{-(y-s)^{2} / 4(\tau-t)} e^{-(x-y)^{2} / 4 \tau} d y d \alpha(\$ 2,115)
\end{aligned}
$$

where the order of integration is interchanged by use of Tonelli's theorem. Next, since the function $e^{-(x-s)^{2} / 4 \tau}$ is bounded and continuous, it follows from Lebesgues' limit theorem that the inner integral of (2.115) converges to $e^{-(x-s)^{2} / 4 \tau}$ as $t \nearrow \tau$, see [123] (p. 61). Consequently, it follows from (2.115) that

$$
W_{\tau}\left[\lim _{t \nearrow \tau} e^{-t \mathcal{D}^{2}} f(x)\right]=c \int_{-\infty}^{\infty} e^{-(x-s)^{2} / 4 \tau} d \alpha(s)=f(x),
$$

where the last step follows by assumption (cf. (2.111)). Thus, (2.114) holds and we conclude.

\section{The inverse of a completely monotonic function}

Lemma 2.45. Let $f: \mathbb{R}_{+} \rightarrow \mathbb{R}_{+}$be a $C M$ function and let $f^{(-1)}: \mathbb{R}_{+} \rightarrow \mathbb{R}_{+}$denote its inverse. Then, it holds that $f^{(-1)} \geq 0, f_{y}^{(-1)} \leq 0$ and $f_{y y}^{(-1)} \geq 0$.

In general, the function $f^{(-1)}$ is, however, not a CM function. In particular, this fact holds true also for $C M$ functions which are represented by (2.81) for some measure $\mu \in \mathcal{B}$.

Notable exceptions to the last statement in the above result, that is to say functions which are $\mathrm{CM}$ and posses $\mathrm{CM}$ inverses, are given by

$$
f(x)=e^{-r x} \quad \text { or } \quad f(x)=(A+B x)^{-r}, \quad r>0 .
$$

We will return to these functions in Example 2.35 below.

Proof. It holds that $f\left(f^{(-1)}(y)\right)=y$. Differentiation of this expression with respect to $y$ yields

$$
f_{x}\left(f^{(-1)}\right) f_{y}^{(-1)}=1
$$


where the functions $f^{(-1)}, f_{y}^{(-1)}$ etc. are evaluated at $y$ and where we use the notation

$$
f_{y}^{(-1)}:=\frac{\partial}{\partial y} f^{(-1)}(y)
$$

By rearrangement and then, in turn, further differentiation, we obtain

$$
\left\{\begin{array}{l}
f_{y}^{(-1)}=\frac{1}{f_{x}\left(f^{(-1)}\right)}, \\
f_{y y}^{(-1)}=-\frac{f_{x x}\left(f^{(-1)}\right) f_{y}^{(-1)}}{\left[f_{x}\left(f^{(-1)}\right)\right]^{2}}=-\frac{f_{x x}\left(f^{(-1)}\right)}{\left[f_{x}\left(f^{(-1)}\right)\right]^{3}}
\end{array}\right.
$$

where, as above, the argument $y$ is suppressed. Thus, as $f$ is CM it follows that $f^{(-1)} \geq 0$, $f_{y}^{(-1)} \leq 0$ and $f_{y y}^{(-1)} \geq 0$.

The third derivative of $f^{(-1)}$ is obtained by further differentiation and given by

$$
\begin{aligned}
f_{y y y}^{(-1)} & =\frac{3 f_{x x}\left(f^{(-1)}\right) f_{x x}\left(f^{(-1)}\right) f_{y}^{(-1)}}{\left[f_{x}\left(f^{(-1)}\right)\right]^{4}}-\frac{f_{x x x}\left(f^{(-1)}\right) f_{y}^{(-1)}}{\left[f_{x}\left(f^{(-1)}\right)\right]^{3}} \\
& =\frac{3 f_{x x}\left(f^{(-1)}\right)^{2}-f_{x x x}\left(f^{(-1)}\right) f_{x}\left(f^{(-1)}\right)}{\left[f_{x}\left(f^{(-1)}\right)\right]^{5}} .
\end{aligned}
$$

In order to show that $f$ being $\mathrm{CM}$ does not imply that $f_{y y y}^{(-1)} \leq 0$, we consider a counterexample. For example, one could consider $g(x)=e^{-x}+e^{-5 x}-2$, which clearly is CM and whose inverse is not CM. However, in order to verify also the last claim of the proposition, define the function $g: \mathbb{R}_{+} \rightarrow \mathbb{R}_{+}$by

$$
g(x)=x^{-1}+x^{-c},
$$

for some constant $c>0$. The function $g$ is clearly CM and given by (2.81) for some measure $\mu$. Direct (but tedious) calculations yields

$$
3 g_{x x}(x)^{2}-g_{x x x}(x) g_{x}(x)=x^{-4-2 c}\left[c^{2}(1+c)(1+2 c)+x^{c-1}\left(6 x^{c-1}-c\left(c^{2}-9 c-4\right)\right)\right]<0,
$$

where the last inequality follows, for example, for the choice of $c=1001$ and $\hat{x}=10^{7 / 1000}$. As $\hat{x}>0$, there exists some $\hat{y}$ such that $g^{(-1)}(\hat{y})=\hat{x}$, which in combination with (2.117) implies that $g_{y y y}^{(-1)}(\hat{y}) \geq 0$. Consequently, the function $g^{(-1)}$ is not $\mathrm{CM}$ and we conclude.

As a final remark, it should be noted that since $f$ is a CM function, we know (cf. page 167 in [120]) that

$$
f_{x x}(x)^{2}-f_{x x x}(x) f_{x} x \leq 0, \quad x \in \mathbb{R}_{+} .
$$

This fact, however, gives us no information about the sign of (2.117). 


\section{Chapter 3}

\section{Optimal investment and consumption for forward stochastic utilities}

\subsection{Connection of forward performance criteria with the classical utility maximization}

Forward performance criteria were introduced in [94, 96, 97] as a complementary notion for the study of optimal portfolio choice. A closely related notion was, independently, introduced and studied in $[61,62]$. The novelty of the forward criterion is its capability to capture dynamically changing preferences and arbitrary trading horizons. In this section, we provide a discussion of the forward investment notion and some of the results to date. The section does not contain any original material but cites various results (see references below). The discussion is formal, for our aim is to provide intuition for the notion of forward performance, relate it to the classical utility maximization problem and establish some links to results in previous and forthcoming chapters.

Forward performance criteria are defined in Section 3.1.1. In Section 3.1.2, the notion of forward propagation of preferences is illustrated with a comparison to an inverse version of the (stochastic) utility maximization problem. In Section 3.1.2.1, some further remarks on the use of stochastic utility functions and the specification of initial preferences are given. In Section 3.1.3, the comparison between the classical and the forward problems is further investigated. We study this comparison within a Brownian market framework where the problems can be related to backward and forward SPDEs, respectively (this is based on previous work in [38, 39, 89, 97]). In Section 3.1.4 some important and illustrative examples are presented. In particular, the so-called time-monotone criteria which are closely linked to the utility functions introduced 
in Section 2.2 and the more general time-monotone criteria studied in Sections 3.2 and 4.4, respectively. For references on the forward investment performance measurement we refer the reader to $[8,96,98,99]$.

\subsubsection{Definition and general remarks}

Within a given market model where $\mathcal{A}$ denotes the set of admissible investment strategies, forward performance criteria are defined as follows.

Definition 3.1. An $\mathcal{F}_{t}$-progressively measurable process $U_{t}(x)$ is a forward performance if, for $t \geq 0$ and $x \in \mathbb{R}_{+}$:

i) the mapping $x \rightarrow U_{t}(x)$ is strictly concave and increasing,

ii) for each $\pi \in \mathcal{A}, \mathrm{E}\left[U_{t}\left(X_{t}^{\pi}\right)\right]^{+}<\infty$, and

$$
\mathrm{E}\left[U_{t}\left(X_{t}^{\pi}\right) \mid \mathcal{F}_{s}\right] \leq U_{s}\left(X_{s}^{\pi}\right), \quad s \leq t,
$$

iii) there exists $\pi^{*} \in \mathcal{A}$ for which equality holds in (3.1) for all $0 \leq s \leq t$.

We continue with a preliminary connection with the classical expected utility problem. To this end, we recall that for the classical utility maximization problem, a utility function $U(x)$ and a terminal horizon $T$ are both fixed. Then, the value-process is defined as

$$
V_{t}(x):=\underset{\pi \in \mathcal{A}}{\operatorname{ess} \sup } \mathrm{E}\left[U\left(X_{T}^{\pi}\right) \mid \mathcal{F}_{t}, X_{t}=x\right] .
$$

In general, the process $V_{t}(x)$ satisfies the so-called martingale optimality principle (cf. [37]). Namely, the value process evaluated with respect to a wealth-process is a super-martingale for every admissible strategy and a martingale at optimum. The second condition in the above definition is then an extension of this property to the entire positive line. Consequently, forward criteria might be viewed as value processes defined for all positive times. However, while timeconsistency in the standard problem is a consequence of the problem formulation, it is here taken as a starting point. Indeed, the notion characterizes the set of all time-consistent preferences and describe their evolution over time. Note that on any fixed finite interval $[0, T)$, a given forward criterion $U_{t}(x)$ coincides with the value function (cf. (3.2)) associated with the (stochastic) terminal utility function $U(x)=U_{T}(x)$ set at the horizon $T$. Moreover, the optimal investments associated with the respective problems coincide on that interval. Forward criteria are therefore closely related to utility maximization with respect to stochastic utility functions.

Contrary to the finite-horizon case, there does not exist any terminal investment end-point and, thus, no (uniformly integrable) terminal element. Hence, while for the finite-horizon case, 
every value-process $V_{t}(x), t \leq T$, is uniquely determined by its terminal utility function, the entire process $U_{t}(x), t \geq 0$, is needed in order to characterize a specific forward criterion. We note that when applied to a specific investor, the idea of the forward formulation is that the investor specifies her initial (as opposed to terminal) preferences and then propagates these forwards in time in a time-consistent manner. That is to say, in accordance with some forward criterion.

A crucial feature of the notion is that there might exist multiple forward criteria with coinciding initial conditions. This property is further illustrated next.

\subsubsection{Propagation of initial preferences and multiplicity of criteria}

For a thorough motivation and axiomatic approach to forward criteria, we refer to [98], [127] and the references therein. Here, the aim is to illustrate some features via a comparison with (an inverse formulation) of the standard utility maximization problem. Specifically, we focus on the mechanisms and the rationality behind the forward propagation of preferences and, furthermore, the fact that the a priori specification of the initial preferences combined with the stochasticity of the criteria yields multiplicity of admissible criteria.

- The inverse investment problem and the associated non-uniqueness for stochastic utility functions

In general, an inverse investment problem refers to a problem where the utility function a priori is unknown while some other quantities, which normally are obtained as an output of the utility maximization problem, are known. The problem in then to infer the former from the latter. While the utility function is hard to specify, investor's actual investments are observables in the market. Inverse investment problems have, therefore, attracted attention in order to shed light on investors' choices; see, among others, [28] and the references therein. Herein, the inverse problem refers to the one where the initial preference profile $u_{0}(x)$ (cf. $V_{0}(x)$ in $(3.2)$ ) is given and the horizon $T$ is fixed (see Section 3.1.2.1 for the specification of this input). Then, the aim is to investigate whether there exists a utility function $U(x)$ such that its value-function coincides with $u_{0}(x)$ at time $t=0$ and, if so, to infer that utility function.

This inverse problem is equivalent to the problem of extending an investment problem in the sense of Definition 2.31. Consequently, for the log-normal market model and under the assumption that the utility function is deterministic, this question was addressed in Section 2.2. Specifically, the utility function corresponding to a given initial profile was inferred and proven to be unique. For general market models, the study of the inverse problem is more complex. Nevertheless, the salient point is that if the assumptions on $U(x)$ are relaxed in that one also allows for stochastic 
utility functions, additional flexibility is obtained and, for a given horizon, there might exist multiple utility functions consistent with a given initial preference profile $u_{0}(x)$. Allowing the investor's preferences to lie within the class of stochastic utility functions - as opposed to the deterministic ones - weakens the assumptions on the investors preferences. The associated inverse problem therefore amounts to determine whether the given initial preferences are compatible with some utility function within a broader class of functions. Hence, the possibility of multiple solutions; see Section 3.1.3 for a more precise argument.

Using the same arguments, it follows that for a given initial preference profile $u_{0}(x)$, there exist multiple forward criteria for which $U_{0}(x)=u_{0}(x)$. For each admissible initial preference profile, there is therefore a collection of forward criteria characterizing the investment behaviour that is dynamically consistent with the given initial profile. We will see in the sequel how one could characterize this collection.

- Initial preference profiles, propagation of preferences and arbitrary horizons

In order to illustrate the terminology forward propagation of preferences, consider an investor who has specified her initial investment choice. Assume, furthermore, that the investor is determined to invest optimally with respect to some, possibly stochastic, utility function which is consistent with these initial preferences. First, let the investment horizon be fixed. As discussed earlier, as long as the investor has only committed to her initial investment profile, there might exist multiple utility functions which are consistent with that choice. However, when making the subsequent investments, let's say over the next infinitesimal interval, she must further restrict her preferences to lie within some subset of these utility functions, for all of which the associated optimal strategies coincide on that interval. In this way, the investor will step-by-step commit herself to a specific utility function. That is to say, the precise form of the terminal utility is gradually decided upon as time goes by and the investor receives more information, while at the same time acknowledging the commitment made by previous investments. Although the exact form of the utility function is not a priori known, the investments are, a posteriori, optimal in the expected utility sense with respect to some stochastic utility function. By use of similar arguments, it follows that investing with respect to a forward criterion is equivalent to gradually specifying the preferences in such a way that the preferences are propagated forwards in a timeconsistent manner. The difference is, that within the forward context, neither the utility nor the horizon is a priori known. However, a posteriori, it holds that the investor has invested optimally with respect to some horizon and some stochastic utility function.

Naturally, this raises the question of how the information flow is incorporated into the gradual specification of the criterion. We note that there are two levels to this specification. On the one 
hand, a given forward criterion is a stochastic process and the specific form of the utility therefore evolves according to the arrival of new information. However, in addition to that, the agent also has the freedom to chose which forward criterion to follow. Indeed, as described above, this choice may be made gradually allowing for certain ambiguity. Exactly how this choice should be restricted in order to reflect the investors preferences is subject to ongoing research. We return to this central question within the Brownian framework below (cf. p 68). We note, however, that the choice can be restricted, for example, in that the criterion is required to be adapted to some smaller filtration (cf. Section 3.1.4.1) or satisfy specific additional dynamical requirements (cf. Section 3.1.4.2). Such additional requirements may even imply that there is a unique way of propagating the preferences. Under such additional assumptions, the requirement that the preferences should propagate forwards to arbitrary horizons may also yield strong restrictions on the set of admissible initial conditions; see further discussion and examples in Sections 3.1.3 and 3.1 .4 , respectively.

Remark 3.2. The fact that the identification of a forward criterion is equivalent to the successive extension of the investment problem as described above, crucially, relies on the fact that the expected utility maximization problem itself is time-consistent. Indeed, for investment criteria which are not time-consistent, such as mean-variance criteria, this principle breaks down. Given the extensive literature on time-consistent versions of continuous time mean-variance criteria (see, among others, [14, 30]) it would be of interest to see to what extent these results could be combined with the above understanding of extended investment horizons. We also note that the relation between mean-variance criteria and forward criteria has been explicitly studied in [117].

\subsubsection{Stochastic utility functions and the specification of initial preferences}

The notion of forward criteria is closely related to the use of stochastic utility functions and the specification of initial, as opposed to, terminal preferences. Next, a few clarifying facts on the use of the former and the specification of the latter are presented.

- On the use of stochastic utility functions

The use of stochastic utility functions is well suited to describe investors' preferences in a number of situations. In particular, they naturally arise when studying the problem of indifference pricing or the use of different numeraires (benchmarking). The use of stochastic utility functions also plays a crucial role when there is uncertainty about the market model or the investor's preferences. Indeed, when the investor has different views or is uncertain about the market model, she might effectively invest with respect to a market model different from the reference model associated with the measure $\mathbb{P}$. Under some assumptions, such a behaviour is equivalent to investing 
according to the reference model but using a specific stochastic utility function (cf. Chapter 5). Moreover, to invest with respect to a deterministic utility function set at some terminal date $T$, is in fact, on the interval $[0, \tau), \tau<T$, equivalent to invest according to some specific stochastic utility function (the value function) set at the horizon $\tau$. Hence, stochastic utility functions are also important within the context of horizon misspecification. Above all, stochastic utility functions enable modelling of possibly state-dependent risk-attitudes. For an axiomatic approach to state-dependent preferences we refer to [98] and the references therein.

- On the specification of the initial preferences

Forward propagation of preferences is based on the assumption that the investor has specified her initial preferences. This is an assumption which might need further motivation. Indeed, in the standard utility maximization problem, the preferences are specified via the investor's horizon and von Neumann-Morgenstern utility function at the end of it. Then, in turn, the optimal investment strategy and the initial preferences are obtained as the solution to that problem. Consequently, the investor is advised how best to invest given her specific individual preferences. This has been the traditional way to pose the problem and, thus, the a priori specification of the initial preferences might seem counter-intuitive.

In reality, however, it is very hard for the investor to specify her horizon and the associated utility function. Nevertheless, investors do invest and their initial preferences can, thus, be viewed as observables in the market. Indeed, the investor can be asked to specify her initial investment profile $\pi_{0}(x), x>0$, which (at least for some models) is directly related to the initial preferences $u_{0}(x), x>0$. Hence, given the difficulties specifying investors' preferences, it is natural to study these actual investment choices to see whether they are in accordance with some preferences and, if so, to analyse and characterize the latter. This motivates the study of different types of inverse problems and, in particular, the version studied here. We note that inverse investment problems were first studied by Black [16, 15] and He and Huang [59]; for recent work we refer to [28] and the reference therein.

The specification of the initial preferences is also relevant from a commitment point of view. Indeed, once the investor has started to invest she is tied to her decisions to date and might wish to act in a way which is consistent with those. Of course, although an investor is asked to specify her entire initial preference profile $\pi_{0}(x), x \geq 0$, she will in fact only invest according to $\pi_{0}\left(x_{0}\right)$, where $x_{0}$ is her initial capital. Hence, commitment to the entire initial investment profile might seem too strong a requirement. However, the initial preference profile might be seen as a proxy for prior investments (cf. the discussion of extending the investment problem in Section 2.2.3 and the results on the recovering the utility functions from investments along a path in [36]). 
To require future investments to be consistent with a given initial preference profile therefore amounts to requiring them to be consistent with prior investments.

\subsubsection{Volatility of the forward process and its connection with the classical formulation}

In this section we relate the above discussion to associated Stochastic Partial Differential equations, originally introduced in [89] and [97] and also studied in [38, 39] (see further comments below). The study of these equations will illustrate a number of important facts and effectively intertwine the notions of the classical utility maximization problem, the inverse formulation thereof and the forward criteria. The relevant SPDEs can, in fact, be derived for more general models (cf. [89]) but we limit ourselves to a Brownian framework.

Consider a filtered probability space $\left(\Omega, \mathcal{F},\left(\mathcal{F}_{t}\right)_{t \geq 0}, \mathbb{P}\right)$ which supports a standard $d$-dimensional Brownian motion $W_{t}, t \geq 0$. Assume that the filtration coincides with the one generated by that Brownian motion. The market consists of a riskless bond with zero interest rate and $k$ risky assets $S_{t}^{i}, t \geq 0, i=1, \ldots, k$, with dynamics specified by

$$
d S_{t}^{i}=S_{t}^{i}\left(\mu_{t}^{i} d t+\sigma_{t}^{i} \cdot d W_{t}\right)
$$

where the coefficients $\mu_{t} \in \mathbb{R}^{k}$ and $\sigma_{t} \in \mathbb{R}^{d \times k}, t \geq 0$, are $\mathcal{F}_{t^{-}}$adapted processes. The market price of risk vector, $\lambda_{t} \in \mathbb{R}^{d}$, is, in turn, defined by $\lambda_{t}:=\left(\sigma_{t}^{T}\right)^{+} \mu_{t}$, where $\left(\sigma_{t}^{T}\right)^{+}$denotes the Moore-Penrose pseudo-inverse of the matrix $\sigma_{t}^{T}$. In particular, it holds that

$$
\sigma_{t}^{T} \lambda_{t}=\mu_{t}
$$

In this market environment, an investor chooses an investment strategy $\pi_{t}$ which is a progressively measurable process denoting the amount invested in the risky assets at time $t$. The associated wealth process $X_{t}^{\pi, c}, t \geq 0$, is given by

$$
X_{t}^{\pi, c}=x+\int_{0}^{t} \sigma_{s} \pi_{s} \cdot\left(\lambda_{s} d s+d W_{s}\right),
$$

where $x$ denotes the initial endowment. We restrict the choice to investment strategies such that the above wealth process is well defined and remains positive at all times.

As for now, let $U(x, t)$ refer to either of the following two objects: a value-process associated with a standard utility maximization problem (defined in (3.2) for $0 \leq t \leq T$ ) or a forward criterion (defined in Definition 3.1 for $t \geq 0$ ). Given the Brownian framework, for both cases it is natural to assume that the parameter-dependent process $U(x, t)$ admits the Itô-decomposition

$$
d U_{t}(x)=b(x, t) d t+a(x, t) \cdot d W_{t},
$$


for some predictable and wealth-dependent processes $a$ and $b$.

The crucial point is that in order for the process $U(x, t)$ to satisfy the martingale optimality principle - for $U\left(X_{T}^{\pi}, t\right)$ to be a super-martingale for every strategy and a martingale at optimum - the dynamics of of this parameter-dependent process must satisfy (cf. below),

$$
d U(x, t)=\frac{1}{2} \frac{\left|\lambda_{t} U_{x}(x, t)+\sigma_{t} \sigma_{t}^{+} a_{x}(x, t)\right|^{2}}{U_{x x}(x, t)} d t+a(x, t) \cdot d W_{t} .
$$

That is to say, the drift-process $b(x, t)$ is uniquely determined in terms of the volatility-process $a(x, t)$. For the classical investment problem, this dynamical requirement is complemented by a terminal condition which yields a Backward SPDE (cf. equation (3.10) below). The definition of forward criteria imposes instead a complementary initial condition which yields a forward SPDE (cf. equation (3.12) below).

Before turning to these specific equations, we formally derive the dynamical requirement in (3.6). This is done by exploiting the martingale optimality principle, which is also what links the value-function to the Hamilton-Jacobi-Bellman equation within a stochastic factor framework. In particular, under appropriate specifications, the Backward SPDE (3.10) reduces to the standard HJB-equation; cf. Section 3.1.4.1 below. However, while the derivation of the HJB-equation makes use of Itô's formula, the present non-Markovian framework requires Itô-Ventzell's formula. Formally, application of the latter yields

$$
\begin{aligned}
d u\left(X_{t}, t\right)= & u_{x}\left(X_{t}, t\right) \sigma_{t} \pi_{t} \cdot\left(\lambda_{t} d t+d W_{t}\right)+\frac{1}{2} u_{x x}\left(X_{t}, t\right)\left|\sigma_{t} \pi_{t}\right|^{2} d t \\
& +b\left(X_{t}, t\right) d t+a\left(X_{t}, t\right) \cdot d W_{t}+\sigma_{t} \pi_{t} \cdot a_{x}\left(X_{t}, t\right) d t
\end{aligned}
$$

Rearrangement of terms then yields

$$
\begin{aligned}
d u\left(X_{t}, t\right)= & \frac{1}{2} u_{x x}\left(X_{t}, t\right)\left(\left|\sigma_{t} \pi_{t}\right|^{2}+2 \sigma_{t} \pi_{t} \cdot \frac{\lambda_{t} u_{x}\left(X_{t}, t\right)+\sigma_{t} \sigma_{t}^{+} a_{x}\left(X_{t}, t\right)}{u_{x x}\left(X_{t}, t\right)}\right) d t \\
& +b\left(X_{t}, t\right) d t+\left(u_{x}\left(X_{t}, t\right) \sigma_{t} \pi_{t}+a\left(X_{t}, t\right)\right) \cdot d W_{t}
\end{aligned}
$$

where $\sigma^{+}$denotes the Moore-Penrose pseudo-inverse and where the quantity $\sigma \sigma^{+}$was introduced as it might be that ${ }^{1} a_{x} \notin \operatorname{Lin}(\sigma)$.

It follows readily that the drift-term attains its maximum for $\pi^{*}$ given in feedback form by

$$
\pi^{*}(x, t)=-\sigma_{t}^{+} \frac{\lambda_{t} U_{x}(x, t)+a_{x}(x, t)}{U_{x x}(x, t)},
$$

\footnotetext{
${ }^{1}$ Indeed, it holds that $\sigma \pi \cdot \sigma \sigma^{+} a_{x}=\pi^{T} \sigma^{T} \sigma \sigma^{+} a_{x}=\pi^{T} \sigma^{T} a_{x}=\sigma \pi \cdot a_{x}$ and, trivially, $\sigma \sigma^{+} a_{x} \in \operatorname{Lin}(\sigma)$. Alternatively, the result might be obtained by rewriting the first drift-term of (3.7) as

$$
\ldots=\left.\frac{1}{2} u_{x x}(x, t)\left(\left|\sigma_{t} \pi_{t}+\frac{\lambda_{t} u_{x}(x, t)+a_{x}(x, t)}{u_{x x}(x, t)}\right|^{2}-\left|\frac{\lambda_{t} u_{x}(x, t)+a_{x}(x, t)}{u_{x x}(x, t)}\right|^{2}\right)\right|_{x=X_{t}} .
$$

It then follows that the maximum is obtained for the $\pi$ which is the least-squares solution to the linear system $\sigma_{t} \pi_{t}=-\frac{\lambda_{t} u_{x}(x, t)+a_{x}(x, t)}{u_{x x}(x, t)}$. That is to say, $\pi^{*}$ is given by (3.8).
} 
where the first and second term of the optimal strategy will be referred to as its myopic and non-myopic part, respectively. Substitution of this expression into (3.7) then yields

$$
d u\left(X_{t}, t\right)=\left(-\frac{1}{2} \frac{\left|\lambda_{t} u_{x}+\sigma_{t} \sigma_{t}^{+} a_{x}\right|^{2}}{u_{x x}}+b\right) d t+\ldots d W_{t}
$$

where the argument $\left(X_{t}, t\right)$ is suppressed on the right hand side. In order for $U(x, t)$ to satisfy the martingale optimality principle, the drift-term in (3.9) should vanish. This yields an explicit expression for $b(x, t)$ which then, in turn, substituted back into (3.5) yields the dynamical requirement specified in (3.6).

Next, the specific SPDEs associated with the different investment problems are presented and discussed.

\section{- The classical utility maximization problem}

For the standard utility maximization problem, the value-process, defined for $0 \leq t \leq T$, is required to coincide with the a priori chosen utility function $U(x)$ at the terminal date. Hence, according to the above, the value-process is expected to satisfy the following backward SPDE

$$
d U(x, t)=\frac{1}{2} \frac{\left|\lambda_{t} U_{x}(x, t)+\sigma_{t} \sigma_{t}^{+} a_{x}(x, t)\right|^{2}}{U_{x x}(x, t)} d t+a(x, t) \cdot d W_{t}, \quad U(x, T)=U(x) .
$$

This was established in [89] (see also [97]), where it was rigorously proven that the value process associated with the utility maximization problem (under some assumptions posed on that value process) must satisfy this equation. In consequence, while a priori existence and uniqueness of solutions to equation (3.10) is not established therein, existence and uniqueness follow for utility functions and market parameters such that the associated value-process satisfies certain conditions.

Note that a solution to the backward SPDE (3.10) is a pair of parameter-dependent processes $U(x, t)$ and $a(x, t)$, which satisfy the equation as well as the terminal condition. That is to say, both the value-process itself and the process $a(x, t)$, henceforth referred to as the volatility of the investment criterion, are simultaneously obtained when solving the Backward SPDE (in the same way as the solution to a BSDE consists of a pair of processes). The optimal investment strategy is entirely specified in terms of these processes (cf. (3.8)).

- The inverse investment problem in terms of volatility of solutions to a specific SPDE

The inverse investment problem introduced in Section 3.1.2 investigates, for a given initial investment profile $u_{0}(x)$, whether there exists a utility function $U(x)$ such that the solution to the associated backward $\operatorname{SPDE}(3.10)$ satisfies $U(x, 0)=u_{0}(x)$. As pointed out above, when allowing for stochastic utility functions, there might exist multiple solutions to this problem. In fact, 
this feature is particularly well illustrated by the SPDE (3.10) where the possibility of multiple stochastic solutions is explicitly captured by the presence of the volatility $a(x, t)$. Formally, for a given initial condition $u_{0}(x)$, one may fix an arbitrary (see further discussion below) volatility process $a(x, t)$ and, then, solve the SPDE

$$
d U(x, t)=\frac{1}{2} \frac{\left|\lambda_{t} U_{x}(x, t)+\sigma_{t} \sigma_{t}^{+} a_{x}(x, t)\right|^{2}}{U_{x x}(x, t)} d t+a(x, t) \cdot d W_{t}, \quad U(x, 0)=u_{0}(x),
$$

forwards in time. If the obtained solutions yield $U(x, T)$ within the class of admissible utility functions (for which there is a unique solution to the backward SPDE (3.10); cf. [89]), we easily see that the choice of

$$
U(x):=U(x, T)
$$

is a solution to the inverse investment problem. The salient point is that any volatility process for which this procedure goes through, gives rise to a possibly different solution. Hence, the non-uniqueness of solutions to the inverse problem.

The possibility of multiple solutions to the inverse investment problem is therefore closely related to the appearance of the volatility process $a(x, t)$ in (3.10). It is natural to define a volatility process $a(x, t)$ to be admissible if the associated equation (3.11) admits a unique solution such that $U(x, T)$ is an admissible utility function. Solving the inverse problem is, thus, equivalent to specifying the set of admissible volatility processes. While some classes of admissible volatility processes have been identified (cf. [39] and further remarks below), a general answer to this question is still lacking. This is partially due to the many subtleties associated with equation (3.11). From an SDE-perspective, it is naturally easier to solve the SPDE (3.11) forwards in time (in the same direction as the information evolves) rather than solving the backward SPDE (3.10); cf. solving an SDE and BSDE, respectively. However, from a PDE-perspective, the forward equation is actually more involved than the backward one. The specific case of zero volatility illustrates this fact (cf. Section 3.1.4 below). While equation (3.10) then can be transformed into the standard one-dimensional heat equation, equation (3.11) is related to the backward heat-equation, well-known to be ill-posed.

- Forward Criteria, initial investment choice and volatility of the criterion

Forward criteria are required to satisfy the martingale optimality principle for all times $t \geq 0$ (cf. Definition 3.1). According to the above, in order to constitute a forward criterion $U(x, t)$ is therefore expected to satisfy the SPDE

$$
d U(x, t)=\frac{1}{2} \frac{\left|\lambda_{t} U_{x}(x, t)+\sigma_{t} \sigma_{t}^{+} a_{x}(x, t)\right|^{2}}{U_{x x}(x, t)} d t+a(x, t) d W_{t}, \quad t \geq 0 .
$$

The associated optimal strategy is then given by (3.8) for $t \geq 0$. This was first established in [97], where the above SPDE was introduced and used for the study of forward criteria. The 
SPDE (3.12) has also been studied in terms of its dual counterpart in [38, 39]. Note that the equation is not, a priori, equipped with any initial condition. Indeed, as a notion, forward criteria characterizes all dynamically consistent preferences. For a Brownian filtration, that corresponds to all parameter-dependent processes which are well-defined solutions to (3.12) for $t \geq 0$. These may, equivalently, be specified via the set of admissible initial conditions and then, in turn, the thereto associated solutions.

In consequence, characterizing forward criteria amounts to solving equation (3.11) with the difference being that the SPDE is now to be satisfied for all positive times. In analogy with the inverse problem, there exist multiple forward criteria associated with a given initial condition and for each admissible initial condition $u_{0}(x)$, there is a one-to-one correspondence between associated forward criteria and admissible volatility processes $a(x, t), t \geq 0$. Here, the latter are those $a(x, t)$ for which the SPDE (3.11) admits a unique solution which remains an admissible utility functions in its spatial variable for all times $t \geq 0$. We note that while a general characterization of these volatility processes is still lacking, specific classes of admissible volatilities have been obtained in $[39,95,99,129]$.

Next, we return to the above question of how the time-unfolding specification of the preferences is to be determined. Note that within the Brownian framework, the way the information flow is incorporated into the preferences is captured by the volatility process. Indeed, once the investor has specified an initial investment profile, the idea is to propagate these preferences forwards in a time-consistent manner. While there is no unique way of doing this, the volatility process is exactly what specifies the way the individual investor's preferences evolve. The gradual specification of the preferences is, therefore, equivalent to the gradual choice of the volatility process $a(x, t)$. We stress that although the flexibility obtained via this choice is one of the novelties of the forward notion, additional requirements which narrow the choice are necessary in order to obtain meaningful solutions for different applications. As was shown in [39], (under certain conditions) there will otherwise exist a forward criterion making any optimal strategy consistent with a given initial condition. Exactly how to narrow the choice in a way that reflects the investor's preferences, is a challenging question which is subject to ongoing research. We conclude this section by considering two specific additional requirements which, respectively, limit the choice to the extent that only a unique criterion remains. We note that under these assumptions, the requirement that the SPDE (3.11) admits a solution for all positive times, strongly restricts the set of admissible initial conditions. 


\subsubsection{Additional requirements on the forward criteria}

As illustrated above, in general, there might exist multiple forward criteria associated with a given initial condition. This allows for the identification of criteria with additional dynamical properties. Here, we present two examples: criteria which are deterministic functions of stochastic factors and criteria with zero volatility. Crucial for both cases is that the SPDE (3.11) reduces to a (possibly random) PDE. Since there is no volatility component present for the latter, the solution associated with a given initial condition is unique (given existence). We stress that the requirement of zero volatility is an example of a property specific for the forward notion. Indeed, for general market models and deterministic utility functions, value functions with this property do not exist.

\subsubsection{Deterministic functions of stochastic factors}

Within the Brownian setting set out above, let $k=1, d=2$, and assume that $\mu_{t}=\mu\left(Y_{t}\right)$ and $\sigma_{t}=$ $\left(\sigma\left(Y_{t}\right), 0\right)^{T}$ for some deterministic functions $\mu(\cdot)$ and $\sigma(\cdot)$, which implies that $\sigma \sigma^{+} a_{x}=\left(a_{x}^{1}, 0\right)^{T}$ and $\lambda_{t}=\left(\mu\left(Y_{t}\right) / \sigma\left(Y_{t}\right), 0\right)^{T}$. Let the stochastic factor $Y_{t}, t \geq 0$, follow the dynamics

$$
d Y_{t}=\mu^{Y}\left(Y_{t}\right) d t+\sigma^{Y}\left(Y_{t}\right) d\left(\rho W_{t}^{1}+\sqrt{1-\rho^{2}} W_{t}^{2}\right)
$$

for some deterministic functions $\mu^{Y}(\cdot)$ and $\sigma^{Y}(\cdot)$. Assume, furthermore, that

$$
U(x, t)=u\left(x, Y_{t}, t\right)
$$

for some deterministic function $u$. We note that this assumption amounts to a restriction in the filtration with respect to which the criterion is required to be adapted. Then, it follows that the dynamics of $U(x, t)$ is given by

$$
d U(x, t)=\left(u_{t}+\mu^{Y}\left(Y_{t}\right) u_{y}+\frac{1}{2} \sigma^{Y}\left(Y_{t}\right)^{2} u_{y y}\right) d t+\sigma^{Y}\left(Y_{t}\right) u_{y} d\left(\rho W_{t}^{1}+\sqrt{1-\rho^{2}} W_{t}^{2}\right)
$$

from where the volatility of $U(x, t)$ is identified as $a(x, t)=\sigma^{Y}\left(Y_{t}\right) u_{y}(x, t)\left(\rho, \sqrt{1-\rho^{2}}\right)$. Then, in turn, equalizing the above drift-term to the drift-term of the SPDE (3.12) for the choice of that specific $a(x, t)$, yields

$$
u_{t}+\mu^{Y}\left(Y_{t}\right) u_{y}+\frac{\sigma^{Y}\left(Y_{t}\right)^{2}}{2} u_{y y}-\frac{1}{2} \frac{\left(\lambda_{t} u_{x}+\sigma^{Y}\left(Y_{t}\right) \rho u_{x y}\right)^{2}}{u_{x x}}=0 .
$$

Hence, the process $u\left(x, Y_{t}, t\right)$ solves the $\operatorname{SPDE}(3.12)$ if the function $u(x, y, t)$ solves equation (3.15). The latter equation might be recognized as the traditional HJB equation at its optimum. However, here to be solved for $t \geq 0$ and equipped with an initial condition. In particular, this equation is ill-posed unless the initial condition is of a very specific form. Forward criteria of this type have been studied in [98] and [99], to which we refer for further details. 


\subsubsection{Time monotone (non-volatile) criteria}

Next, the so-called non-volatile criteria are considered. They are characterized by the requirement that the volatility of the criterion is identically zero. Since such criteria can be verified to be monotone in time, they are also referred to as time-monotone criteria. They were introduced and characterized in $[8,96]$. We refer to these papers for details and limit ourselves to a formal discussion herein, the aim of which is to point out some characteristics and give intuition for this important class of forward criteria. Specifically, we relate to the work in previous and forthcoming chapters. Indeed, while non-volatile criteria turn out to be closely related to the utility functions with completely monotonic inverses studied in Section 2.2, they also constitute a particular case of the non-volatile forward investment-consumption criteria and robust criteria studied, respectively, in Section 3.2 and Chapter 5 below.

- Time-monotonicity, uniqueness and path-wise myopic behaviour

By definition, the volatility process $a(x, t)$ in (3.5) is specified to be identically zero. Hence, one would expect there to be a unique criterion of this type associated with every admissible initial condition (cf. the discussion on page 67 and equation (3.11)). As verified next, this is also the case.

Let $U_{t}(x, t):=b(x, t)$. Then, according to equation (3.12), in order to constitute a forward criterion, $U(x, t)$ needs to be an $\mathcal{F}_{t}$-measurable random function which is a path-wise solution to the equation

$$
U(x, 0)=u^{0}(x), \quad U_{t}(x, t)=\frac{1}{2}\left|\lambda_{t}\right|^{2} \frac{U_{x}(x, t)^{2}}{U_{x x}(x, t)}, \quad t \geq 0 .
$$

According to formula (3.8), the associated optimal strategy is then given by

$$
\pi^{*}(x, t)=-\sigma_{t}^{+} \lambda_{t} \frac{U_{x}(x, t)}{U_{x x}(x, t)} .
$$

A comparison of the above expressions with equation (2.89) (cf. also (3.15)), yields that smooth criteria, path-wise, behave similarly to (deterministic) value-functions within a log-normal model. In particular, the optimal strategy is path-wise myopic. Note, however, that the market model is specified in (3.3), where the coefficients are general $\mathcal{F}_{t}$-progressively measurable processes. That is to say, the market model is not log-normal; in fact, it might even be incomplete. Instead, it is the requirement that the criteria should be smooth that forces them to be of a very specific stochastic form which, in turn, render the behaviour pathwise myopic. More precisely, since equation (3.16) is equipped with an initial condition and to be satisfied for all positive times, it corresponds - pathwise - to the inverse log-normal Hamilton-Jacobi-Bellman equation to be satisfied for all positive times. Consequently, the problem of solving for smooth forward criteria is pathwise equivalent to the problem of extending the log-normal investment-problem with 
respect to deterministic utility functions to arbitrary horizons. Given the link to completely monotonic functions, established for the latter problem in Section 2.2.3, one would expect also time-monotone forward criteria to be related to completely monotonic functions. This is indeed the case. The exact relation is specified next.

- Specification of the criteria and relation to utility functions with completely monotonic inverses

For a given criterion $U(x, t)$, introduce the function $H(y, t)$ via the path-wise transformation

$$
U_{x}(H(y, t), t)=e^{-y+\frac{1}{2} A_{t}},
$$

where the process $A_{t}, t \geq 0$, is given by

$$
A_{t}=\int_{0}^{t}\left|\lambda_{s}\right|^{2} d s
$$

Note that since there is a one-to-one correspondence (up to affine transformations) between random functions $U(x, t)$ and $H(x, t)$, a forward criterion $U(x, t)$ is uniquely specified via its associated function $H(y, t)$. Moreover, it follows (cf. Lemma 3.6 below) that $U(x, t)$ is a path-wise solution to equation (3.16), if and only if, $H(y, t)$ is a path-wise solution to the equation

$$
H(y, 0)=\left(u_{x}^{0}\right)^{(-1)}\left(e^{-y}\right), \quad H_{t}+\frac{\left|\lambda_{t}\right|^{2}}{2} H_{y y}=0 . \quad t \geq 0 .
$$

This equation is, path-wise, to be recognised as the ill-posed backward heat equation to be solved for all times $t \geq 0$. Since we consider the case when the wealth is restricted to remain positive, the set of admissible initial conditions and associated solutions of equation (3.19) can then be obtained directly from the results presented in Section 2.2.3. Indeed, recall that Widder's results on the backward heat-equation upon which the proofs therein relied, in fact, originally were introduced to the study of portfolio optimization in order to study time-monotone forward criteria (see [96] where, in particular, similar results also are obtained for utility functions defined on the entire real line).

Consequently (cf. Theorem 2.32), there exists an admissible solution to equation (3.19) and, thus, to equation (3.16), if and only if $u^{0}$ satisfies that

$$
\left(u_{x}^{0}\right)^{(-1)}\left(e^{-y}, 0\right)=\int_{0}^{\infty} e^{y z} \mu(d z)
$$

for some measure $\mu \in \mathcal{B}$ (cf. page 40). Then, the associated solution to equation (3.19) is given by

$$
H(y, t)=\int_{0}^{\infty} e^{y z-\frac{1}{2} z^{2} A_{t}} \mu(d z)
$$

which, in turn, uniquely determines the associated solution $U(x, t)$ of equation (3.16). Since this process $U(x, t)$ is $\mathcal{F}_{t}$-measurable and it holds that $U(\cdot, t)$ is increasing and concave, it consequently constitutes an admissible time-monotone criterion. 
The above verifies that the restriction to non-volatile criteria (and, thus, the specification of the volatility) indeed implies that there is a unique criterion associated with every admissible initial condition. Note that the specific form of these criteria is closely related to the imposed requirement that forward criteria should be well-defined for all positive times. As pointed out above, for the case of smooth criteria (see also Section 3.1.4.1), this requirement strongly restricts the set of admissible initial conditions.

- A specific path-dependent utility function for a fixed horizon

The fact that time-monotone forward criteria are defined in a very specific way which render the behaviour path-wise myopic, can be exploited in order to define a particular path-dependent utility function with interesting properties. To this end, consider an investor with the fixed terminal horizon $T>0$, at which the preferences are represented by the path-dependent stochastic utility function

$$
U(x):=U(x, T)
$$

where $U(x, t)$ is time-monotone forward criterion of the above type; that is to say, defined via (3.18) and (3.20) for some $\mu \in \mathcal{B}$. Then, at any time $t \leq T$, the value function and optimal strategy are given, respectively, by $V(x, t)=U(x, t)$ and formula (3.17) (cf. the discussion on page 59). Consequently, the choice of this specific path-dependent utility function, implies that the optimal strategy is path-wise myopic. Note that this holds when the asset-dynamics is defined with respect to general processes $\mu$ and $\sigma$ (cf. (3.3)). Indeed, as for the forward case, it is the very specific form of the utility function that renders the specific behaviour.

\subsection{Forward investment and consumption criteria}

In this section, we study the extended notion of forward investment and consumption criteria. Such a study was initiated in [9], where the definition first appeared and several important facts were established. Therein, a general semi-martingale market setting was considered and then, in turn, time-monotone forward criteria were studied under the assumption of a Brownian filtration. Here, we consider a Brownian filtration throughout and the main focus is on establishing explicit characterization and economic interpretation of time-monotone investment-consumption criteria.

Our contribution is threefold. First, we consider criteria with non-zero volatility and formally derive an SPDE which they are expected to satisfy. Although only formally derived, this SPDE will illustrate important aspects of the notion and give intuition for the restriction to timemonotone criteria. Second, we provide a detailed study of the time-monotone forward investmentconsumption criteria. While some of these results appeared already in [9], we provide the details 
of the underlying arguments. Third, we provide explicit formulae for the optimal investment and consumption processes (cf. Proposition 3.13). We also show that a specific class of timemonotone preferences, in fact, correspond to a deterministic compilation of classical infinite horizon Merton and pure forward investment criteria (cf. Proposition 3.14). This provides economic interpretation and illustrates that forward investment-consumption criteria constitute a generalisation of infinite horizon the Merton criteria, which allows the investor to consider utility from investments as well as consumption.

\subsubsection{Definition of forward investment-consumption criteria}

We consider the same market model as in Section 3.1.3. However, the investor now chooses a consumption stream $c_{t}$ as well as an investment strategy $\pi_{t}, t \geq 0$. These are progressively measurable processes denoting the amount consumed and invested, respectively, at time $t$. Hence, the associated wealth process in discounted units, $X_{t}^{\pi, c}, t \geq 0$, is given by

$$
X_{t}^{\pi, c}=x+\int_{0}^{t} \sigma_{s} \pi_{s} \cdot\left(\lambda_{s} d s+d W_{s}\right)-\int_{0}^{t} c_{s} d s
$$

where $x$ denotes the initial endowment. We restrict the choice to investment and consumption pairs such that the above wealth process is well defined and remains positive at all times.

The investor considers a portfolio selection criterion of a type referred to as forward investment and consumption criteria. Following [9], we consider the following Definition.

Definition 3.3. A pair of adapted functions $\left(u, u^{c}\right)$ defined on $\mathbb{R}_{+} \times[0, \infty) \times \Omega$ is a forward investment and consumption criterion if

i) $u(\cdot, t, \omega)$ and $u^{c}(\cdot, t, \omega)$ are increasing and concave,

ii) for all $0 \leq t \leq T$ and $\mathcal{F}_{t}$-measurable $\xi$,

$$
u(\xi, t) \geq \mathrm{E}\left[u\left(X_{T}^{\pi, c}, T\right)+\int_{t}^{T} u^{c}\left(c_{s}, s\right) d s \mid \mathcal{F}_{t}\right], \text { a.s. }
$$

for all admissible investment and consumption strategies $\pi$ and $c$ such that $X_{t}^{\pi, c}=\xi$,

iii) equality holds in (3.22) for some admissible pair $\pi^{*}$ and $c^{*}$.

As holds for the pure investment case (cf. Section 3.1.1), on the finite interval $[0, T$ ), the investor considering a given forward investment-consumption criterion $u(x, t)$ and $u^{c}(c, t)$ will invest and consume in the same way as does the investor considering the fixed terminal horizon $T$ and (in a standard manner) optimizing utility from consumption and terminal wealth with respect to the (possibly stochastic) felicity and utility function given by

$$
U^{c}(x, t):=u^{c}(x, t ; \omega) \quad \text { and } \quad U_{T}(x):=u(x, T ; \omega),
$$


respectively. In particular, it then holds that the process

$$
u\left(X_{t}^{\pi, c}, t\right)+\int_{0}^{t} U^{c}\left(c_{s}, s\right) d s, \quad 0 \leq t \leq T,
$$

is a super-martingale for every admissible pair $(\pi, c)$ and a martingale for the optimal one $\left(\pi^{*}, c^{*}\right)$. Hence, $u(x, t), t \leq T$, coincides with the value-function for the investment problem associated with (3.23). This illustrates that for general forward investment-consumption criteria, $u(x, t)$ and $u^{c}(c, t)$ can also be thought of as value and felicity-functions, respectively.

Moreover, note that the standard infinite horizon Merton problem constitutes a particular case of forward investment consumption criteria. Indeed, for a given felicity function $u^{c}(c, t)$, define the associated value function by

$$
u(x, t)=\sup _{\pi, c} \mathrm{E}\left[\int_{t}^{\infty} u^{c}\left(c_{t}, t\right) d s \mid \mathcal{F}_{t}\right] .
$$

Then, it immediately follows that the pair $u(x, t)$ and $u^{c}(c, t)$ represents a forward investmentconsumption criterion in the sense of Definition 3.3. Note, however, that general forward investment-consumption criteria need not be of this form. Indeed, the aim of the notion is to quantify preferences on arbitrary or infinite horizons, when the investor does not only receive utility from consumption but also from the investments themselves.

\subsubsection{Forward investment consumption criteria within a Brownian fil- tration and the associated SPDE}

In this section we derive an SPDE which forward investment-consumption criteria are expected to satisfy within a Brownian filtration. This SPDE is closely related to the one derived for the pure investment case under appropriate regularity and integrability conditions (cf. [89, 97] and Section 3.1.3) and is obtained by use of the same arguments. Apart from being of interest in its own right, this SPDE will illustrate and provide intuition for some specific features of forward investment-consumption criteria and underline the differences to standard maximization of utility from consumption.

Given that the filtration is Brownian, we impose the additional assumption (cf. (3.5)) that $u(x, t)$ admits the Itô decomposition

$$
d u(x, t)=b(x, t) d t+a(x, t) \cdot d W_{t}, \quad t \geq 0,
$$

for some (wealth-dependent) coefficients $a$ and $b$ which are $\mathcal{F}_{t}$-progressively measurable processes. Then, the Itô-Ventzell formula might formally be applied in order to obtain (cf. equation (3.7) 
and footnote 1) the following dynamics

$$
\begin{aligned}
d u\left(X_{t}, t\right)+u^{c}\left(c_{t}, t\right)= & {\left[\frac{1}{2} u_{x x}\left(X_{t}, t\right)\left(\left|\sigma_{t} \pi_{t}\right|^{2}+2 \sigma_{t} \pi_{t} \cdot \frac{\lambda_{t} u_{x}\left(X_{t}, t\right)+\sigma_{t} \sigma_{t}^{+} a_{x}\left(X_{t}, t\right)}{u_{x x}\left(X_{t}, t\right)}\right)\right.} \\
& \left.+b\left(X_{t}, t\right)+u^{c}\left(c_{t}, t\right)-u_{x}\left(X_{t}, t\right) c_{t}\right] d t+\left[u_{x}\left(X_{t}, t\right) \sigma_{t} \pi_{t}+a\left(X_{t}, t\right)\right] \cdot d W_{t}
\end{aligned}
$$

where, as before, the quantity $\sigma \sigma^{+}$was introduced as it might be that $a_{x} \notin \operatorname{Lin}(\sigma)$. It follows readily that the drift-term attains its maximum for $\pi^{*}$ and $c^{*}$ given in feedback form by

$$
\pi^{*}(x, t)=-\sigma_{t}^{+} \frac{\lambda_{t} u_{x}(x, t)+a_{x}(x, t)}{u_{x x}(x, t)} \quad \text { and } \quad c^{*}(x, t)=I^{c}\left(u_{x}(x, t), t\right),
$$

where the first and second term of the optimal strategy will be referred to as its myopic and non-myopic part, respectively. Substitution for these expressions into (3.25) yields

$$
d u\left(X_{t}, t\right)+u^{c}\left(c_{t}, t\right)=\left[-\frac{1}{2} \frac{\left|\lambda_{t} u_{x}+\sigma_{t} \sigma_{t}^{+} a_{x}\right|^{2}}{u_{x x}}+b+\hat{u}^{c}\left(u_{x}, t\right)\right] d t+\ldots d W_{t},
$$

where the argument $\left(X_{t}, t\right)$ is suppressed on the right hand side. Recall that $u(x, t)$ and $u^{c}(x, t)$ constitute a forward criterion if $u\left(X_{t}^{\pi}, t\right)+\int_{0}^{t} u^{c}\left(c_{s}, s\right) d s$ is a super-martingale for every admissible strategy and a martingale at optimum. Hence, the drift-term of (3.27) should vanish. This condition yields an explicit expression for $b(x, t)$ which, in turn, substituted into (3.24) yields

$$
d u(x, t)=\left[\frac{1}{2} \frac{\left|\lambda_{t} u_{x}(x, t)+\sigma_{t} \sigma_{t}^{+} a_{x}(x, t)\right|^{2}}{u_{x x}(x, t)}-\hat{u}^{c}\left(u_{x}(x, t), t\right)\right] d t+a(x, t) \cdot d W_{t}, \quad t \geq 0
$$

Hence, formally, we have arrived at an SPDE which a pair of processes must satisfy in order to constitute a forward investment-consumption criterion. In particular, for the case of no intermediate consumption, that is to say when $u^{c}(x, t) \equiv 0$, the above SPDE reduces to the one presented in Section 3.1.3 (cf. [89] and [97]). Similarly to the pure investment case, the SPDE (3.28) is a priori not equipped with any additional condition. Indeed, the notion of forward investment-consumption criteria (cf. Definition 3.3) aims at incorporating all criteria which describe dynamically consistent investment behaviour when consumption is taken into consideration. However, the closely related question of specifying the set of forward criteria associated with a given initial preference profile - the question of how to propagate investments forwards in time - amounts to the study of the SPDE (3.28) equipped with an initial condition. As for the pure investment case, there does not exist a unique solution to that equation. Here due to volatility of the criterion as well as the presence of the consumption term (cf. further discussion below).

Characterizing the set of admissible initial conditions and the associated solutions to the SPDE (3.28) is, in general, a highly non-trivial task which we do not address here. Rather, we restrict 
the study to criteria which are differentiable with respect to time and get explicit results for this case. Before turning to these criteria, we present another yet another specific example.

Example 3.4. Consider the same model as in Section 3.1.4.1. That is to say, let $k=1, d=2$, and assume that $\mu_{t}=\mu\left(Y_{t}\right)$ and $\sigma_{t}=\left(\sigma\left(Y_{t}\right), 0\right)^{T}$ for some deterministic functions $\mu(\cdot)$ and $\sigma(\cdot)$ and a stochastic factor $Y_{t}, t \geq 0$, with dynamics specified by (3.13). Furthermore, consider criteria such that $u^{c}(c, t)=u^{c}\left(c, Y_{t}, t\right)$ and $u(x, t)=u\left(x, Y_{t}, t\right)$ for some deterministic functions $u^{c}$ and $u$.

Then, it holds that the dynamics of $u(x, t)$ is given by (3.14) from where its volatility $a(x, t)$ might be identified. In turn, equalizing the drift-term of $u(x, t)$ (cf. (3.14)) to the drift-term of the SPDE (3.28) for the choice of that specific volatility $a(x, t)$, yields

$$
u_{t}+\mu^{Y}\left(Y_{t}\right) u_{y}+\frac{\sigma^{Y}\left(Y_{t}\right)^{2}}{2} u_{y y}=\frac{1}{2} \frac{\left(\lambda_{t} u_{x}+\sigma^{Y}\left(Y_{t}\right) \rho u_{x y}\right)^{2}}{u_{x x}}-\hat{u}^{c}\left(u_{x}, t\right) .
$$

Hence, the process $u\left(x, Y_{t}, t\right)$ solves the SPDE (3.28) if the function $u(x, y, t)$ solves equation (3.29). The latter equation might be recognized as the traditional HJB equation - for the consumption and investment problem - at its optimum. However, here to be solved for $t \geq 0$ and equipped with an initial condition, which makes it an ill-posed equation. For the case of no intermediate consumption, forward criteria of this type have been studied in [98, 99].

Note that the above arguments could also be used to deduce (cf. the discussion in Section 3.1.3) a specific backward SPDE which the value-process, associated with the standard finite horizon utility maximization problem where the investor obtains utility from consumption as well as terminal wealth, is expected to satisfy. Specifically, the associated value-process defined for $t \leq T$, is expected to satisfy the $\operatorname{SPDE}(3.28)$ combined with the terminal condition $u(x, T)=U(x)$, where $U(x)$ is the terminal utility function. This equation does not seem to have appeared in the literature. A rigorous treatment could probably be carried out by use of similar arguments to those used for the pure investment case in [89].

\subsubsection{Time-monotone forward investment-consumption criteria}

We restrict the study to criteria $\left(u, u^{c}\right)$ such that $u(x, t)$ is smooth in time. We note that this requirement is posed on the value-function part of the forward investment-consumption criterion, not the felicity function. As it can be shown that smooth criteria actually are monotone in time (cf. [8]), they are henceforth referred to as time-monotone investment-consumption criteria. Similarly to what was done for the pure investment case in Section 3.1.4.2 (cf. [8, 96]), the specific form of the SPDE (3.15) for this case, will be used in order to obtain explicit representations of 
these criteria. This will enable further insights and yield understanding also of general forward investment-consumption criteria.

\subsubsection{The associated random PDE and its primal and dual versions}

Since the volatility of time-monotone forward criteria is required to be zero, that is $a(x, t) \equiv 0$ (cf. (3.24)), the SPDE (3.15) reduces to the following Hamilton-Jacobi-Bellman type equation

$$
u_{t}-\frac{\left|\lambda_{t}\right|^{2}}{2} \frac{u_{x}^{2}}{u_{x x}}+\hat{u}^{c}\left(u_{x}, t\right)=0, \quad t \geq 0, \quad \text { a.s. }
$$

Hence, one would expect that a pair of random functions $\left(u, u^{c}\right)$ constitutes a time-monotone forward criterion if and only if they constitute a path-wise solution to the random equation (3.30). In fact, by use of different methods (they do not consider the associated SPDE), this has been proven to be the case in [9]. We therefore refer thereto for a rigorous proof of this statement and limit ourselves to emphasize the intuitive fact that the restriction to non-volatile criteria implies that the SPDE (3.15) reduces to a random PDE. Consequently, the solution is to satisfy this equation path-wise. In addition to being a path-wise solution to this random PDE, the criterion is also required to be $\mathcal{F}_{t}$-measurable. Hence, the criterion needs to satisfy the same deterministic initial condition for all paths. Characterizing time-monotone criteria is therefore equivalent to specifying the solutions to equation (3.30). The latter problem is, in turn, better addressed within the dual domain, for the convex conjugate of the solution to (3.30) satisfies, in fact, a linear equation (cf. Lemma 3.6 below).

In order to work in the dual domain, some auxiliary quantities are needed. Let the processes $M_{t}$ and $A_{t}, t \geq 0$, which will be of frequent use in the upcoming analysis, be given by

$$
M_{t}=\int_{0}^{t} \lambda_{s} \cdot d W_{s} \quad \text { and } \quad A_{t}=\langle M\rangle_{t}=\int_{0}^{t}\left|\lambda_{s}\right|^{2} d s
$$

Then, in turn, the following auxiliary functions are defined.

Definition 3.5. The convex conjugate $\hat{u}: \mathbb{R} \times[0, \infty) \rightarrow \mathbb{R}_{+}$is defined by

$$
\hat{u}(y, t)=\sup _{x>0}\{u(x, t)-x y\} .
$$

Moreover, the functions $I: \mathbb{R} \times[0, \infty) \rightarrow \mathbb{R}_{+}$and $H: \mathbb{R} \times[0, \infty) \rightarrow \mathbb{R}_{+}$are defined via

$$
u_{x}(I(y, t), t)=y \quad \text { and } \quad u_{x}(H(y, t), t)=\exp \left\{-y+\frac{1}{2} A_{t}\right\}
$$

respectively, where $A_{t}, t \geq 0$ as in (3.31). The functions $\hat{u}^{c}, I^{c}$ and $H^{c}$ are, analogously, defined using $u^{c}$ instead of $u$ in (3.32) and (3.33), respectively. 
Next, we pass to the dual domain in which the equation (3.30) is linearised ${ }^{2}$.

Lemma 3.6. The functions $u(x, t ; \omega)$ and $u^{c}(x, t ; \omega)$ satisfy equation (3.30) if and only if $\hat{u}(y, t ; \omega)$ and $\hat{u}^{c}(y, t ; \omega)$ defined in (3.32), satisfy

$$
\hat{u}_{t}+\frac{\left|\lambda_{t}\right|^{2}}{2} y^{2} \hat{u}_{y y}+\hat{u}^{c}=0, \quad t \geq 0, \quad \text { a.s. }
$$

This, in turn, holds if and only if $H(y, t ; \omega)$ and $H^{c}(y, t ; \omega)$ satisfy

$$
H_{t}+\frac{\left|\lambda_{t}\right|^{2}}{2} H_{y y}+H^{c}=0, \quad t \geq 0, \quad \text { a.s. }
$$

Proof. The supremum in (3.32) is obtained for $x=I(y, t)$ and, thus,

$$
\hat{u}(y, t)=u(I(y, t), t)-y I(y, t) .
$$

Differentiation of this equation yields

$$
\left\{\begin{array}{l}
\hat{u}_{t}=u_{x}(I, t) I_{t}+u_{t}(I, t)-y I_{t}=u_{t}(I, t), \\
\hat{u}_{y}=u_{x}(I, t) I_{y}-I-y I_{y}=-I, \\
\hat{u}_{y y}=-I_{y},
\end{array}\right.
$$

where the functions $\hat{u}$ and $I$ are evaluated at $(y, t)$ and it was used that, according to (3.33), $u_{x}(I(y, t), t)=y$. Moreover, differentiation of (3.33) yields $u_{x x}(I(y, t), t) I_{y}=1$, which implies that

$$
\frac{u_{x}(I(y, t), t)^{2}}{u_{x x}(I(y, t), t)}=y^{2} I_{y}(y, t)
$$

By use of (3.36), equation (3.30) can be rewritten as

$$
u_{t}-\frac{\left|\lambda_{t}\right|^{2}}{2} \frac{u_{x}^{2}}{u_{x x}}-I^{c}\left(u_{x}, t\right) u_{x}+u^{c}\left(I^{c}\left(u_{x}, t\right), t\right)=0 .
$$

Use of (3.37), (3.38) and equation (3.39) evaluated at $x=I(y, t)$, then yields that

$$
\begin{aligned}
\hat{u}_{t}(y, t)+\frac{\left|\lambda_{t}\right|^{2}}{2} y^{2} & \hat{u}_{y y}(y, t)+\hat{u}^{c}(y, t) \\
& =u_{t}(I(y, t), t)-\frac{\left|\lambda_{t}\right|^{2}}{2} y^{2} I_{y}(y, t)+u^{c}\left(I^{c}(y, t), t\right)-y I^{c}(y, t)=0 .
\end{aligned}
$$

In other words, equation (3.30) implies equation (3.34). Next, we differentiate (3.34) to obtain:

$$
\hat{u}_{y t}+\frac{\left|\lambda_{t}\right|^{2}}{2}\left[2 y \hat{u}_{y y}+y^{2} \hat{u}_{y y y}\right]+\hat{u}_{y}^{c}=0 .
$$

Substitution for $I(y, t)=-\hat{u}_{y}(y, t)$ and $I^{c}(y, t)=-\hat{u}_{y}^{c}(y, t)$ (cf. (3.37)) yields that the functions $I(y, t)$ and $I^{c}(y, t)$ satisfy

$$
I_{t}+\left|\lambda_{t}\right|^{2} y I_{y}+\frac{\left|\lambda_{t}\right|^{2}}{2} y^{2} I_{y y}+I^{c}=0
$$

${ }^{2}$ This result is also to be found in [9] (cf. equations (2), (3) and p. 11 therein). However, there given without proof. 
Note that (3.37) in combination with (3.33), yields the relation

$$
\hat{u}_{y}(y, t)=-I(y, t)=-H\left(-\ln y+\frac{1}{2} A_{t}, t\right) .
$$

Differentiation of this expression, then, in turn, yields

$$
\left\{\begin{array}{l}
I_{t}=\frac{\left|\lambda_{t}\right|^{2}}{2} H_{y}+H_{t}, \\
I_{y}=-\frac{H_{y}}{y}, \\
I_{y y}=\frac{1}{y^{2}}\left(H_{y}+H_{y y}\right),
\end{array}\right.
$$

where $I$ is evaluated at $(y, t)$ and $H$ and its derivatives are evaluated at $\left(-\ln y+\frac{1}{2} A_{t}, t\right)$. Note that the relation in (3.41) also holds for $\hat{u}^{c}, I^{c}$ and $H^{c}$. Substitution for these expressions into equation (3.40) therefore yields

$$
\frac{\left|\lambda_{t}\right|^{2}}{2} H_{y}+H_{t}-\left|\lambda_{t}\right|^{2} H_{y}+\frac{\left|\lambda_{t}\right|^{2}}{2}\left(H_{y}+H_{y y}\right)+H^{c}=0,
$$

which reduces to (3.35). The reverse implications follows by similar arguments. We easily conclude.

The specification of time-monotone investment-consumption criteria is consequently reduced to the search for solutions to equation (3.35) which is a linear PDE with random coefficients. Note that it coincides with equation (3.19) except for the fact that the presence of consumption introduces an additional source-term into the equation. We stress that the requirement that $H(y, t) \in \mathcal{F}_{t}$ enforces additional conditions on $H^{c}$ (or, equivalently, on $u^{c}$ ) that restricts the set of admissible solutions. This since the property that $H^{c}(y, t) \in \mathcal{F}_{t}$ itself is not enough to ensure that $H(y, t) \in \mathcal{F}_{t}$.

Remark 3.7 (A utility function which yields myopic investments on the finite horizon). As for the pure investment case (cf. Section 3.1.4.2), a given smooth forward investment-consumption criterion $u(x, t)$ and $u^{c}(c, t)$, can be used to define a specific (stochastic) utility function for the investor who considers the fixed finite horizon $T$ and in a standard manner optimizes utility from consumption and terminal wealth. Indeed, let the stochastic terminal utility and felicity functions, $U_{T}$ and $U^{c}$, be defined via (3.23). The associated value-function is then given by $u(x, t), t \leq T$. That is to say, the value-function is smooth and, thus, the investments pathwise myopic (cf. (3.26)). The result holds for markets defined via (3.3) with respect to general progressively measurable processes $\mu_{t}$ and $\sigma_{t}$. Indeed, it is the particular path-dependent form of $U^{c}$ and $U_{T}$, respectively, that forces the value-function to be smooth in time and thus, in turn, the optimal strategy to be myopic. This should be contrasted to Example 3.4. 


\subsubsection{Explicit characterization of the time-monotone criteria}

In this section we obtain explicit characterizations of the time-monotone forward investmentconsumption criteria. The following additional assumption is imposed on the underlying market model.

Assumption 3.8. The market price of risk $\lambda_{t}, t \geq 0$, is such that the process $A_{t}, t \geq 0$, (cf. (3.31)) satisfies

$$
\lim _{t \rightarrow \infty} A_{t}=\infty, \quad \text { a.s. }
$$

For given functions $H$ and $H^{c}$, the functions $h: \mathbb{R} \times[0, \infty) \times \Omega \rightarrow \mathbb{R}_{+}$and $h^{c}: \mathbb{R} \times[0, \infty) \times \Omega \rightarrow \mathbb{R}_{+}$ are (path-wise) defined via the implicit relations

$$
H(y, t)=h\left(y, A_{t}\right) \quad \text { and } \quad H^{c}(y, t)=\left|\lambda_{t}\right|^{2} h^{c}\left(y, A_{t}\right)
$$

Due to Assumption 3.8, the functions $h$ and $h^{c}$ are then well-defined almost surely. Furthermore, it follows that for $H$ and $H^{c}$ to solve equation (3.35), it is necessary and sufficient that the associated functions $h$ and $h^{c}$, constitute a path-wise solution to the following (backward) inhomogeneous heat-equation (the set of admissible initial conditions is further discussed below):

$$
h_{t}+\frac{1}{2} h_{y y}+h^{c}=0, \quad(y, t) \in \mathbb{R} \times[0, \infty) .
$$

Hence, the search for time-monotone forward criteria is reduced to the search for (random) solutions to this equation. In order to specify these solutions we fix some further notation. To this end, let $k(z, t)$ denote the fundamental solution to the heat equation,

$$
k(z, t)=\sigma(t) \frac{e^{-\frac{z^{2}}{2}}}{\sqrt{2 \pi t}},
$$

where $\sigma(\cdot)$ is the step-function at $t=0$ and we use the notational convention $k(z):=k(z, 1)$. Moreover, let $\left(k * h^{c}\right): \mathbb{R} \times[0, \infty) \times \Omega \rightarrow \mathbb{R}_{+}$be the convolution in $\mathbb{R}^{2}$, that is with respect to space as well as time, of $h^{c}(y, t ; \omega)$ and the fundamental solution $k(y, t)$. That is to say,

$\left(k * h^{c}\right)(y, t):=\int_{t}^{\infty} \int_{-\infty}^{\infty} k(z-y ; s-t) h^{c}(z, s) d z d s=\int_{t}^{\infty} \int_{-\infty}^{\infty} k(z) h^{c}(y+\sqrt{s-t} z, s) d z d s$.

This function is well-known to be a (path-wise) solution to equation (3.45). Also note that $\lim _{t \rightarrow \infty}\left(k * h^{c}\right)(y, t)=0$ a.s. for all $y \in \mathbb{R}$. Moreover, define the function $h^{\mu}: \mathbb{R} \times[0, \infty) \times \Omega \rightarrow$ $\mathbb{R}_{+}$as

$$
h^{\mu}(y, t):=\int_{0}^{\infty} e^{r y-\frac{1}{2} r^{2} t} \mu(d r)
$$


for some random positive Borel measure $\mu$ such that $\int_{\mathbb{R}} e^{r y} \mu(d y)<\infty, y \in \mathbb{R}$, a.s. That is to say, for some measure $\mu \in \mathcal{B}$ with $\mathcal{B}$ given by (note that we redefine $\mathcal{B}$ to denote a bigger set than before, cf. page 40):

$$
\mathcal{B}=\left\{\mu \text { defined on } \mathcal{B}(\mathbb{R}) \times \Omega: \forall B \in \mathcal{B}(\mathbb{R}), \mu(B) \geq 0 \text { and } \int_{\mathbb{R}} e^{r y} \mu(d r)<\infty, y \in \mathbb{R}, \text { a.s. }\right\} .
$$

Recall that according to the result by Widder (cf. [123] and Section 3.1.4.2), every solution to the homogeneous (backward) heat-equation defined for $t \geq 0$ (cf. equation (3.45) with $h^{c} \equiv 0$ ) must be given by (3.47) for some deterministic measure $\mu \in \mathcal{B}$. The next result specifies all solutions to equation (3.45) and, consequently, the set of time-monotone forward investment-consumption criteria.

Theorem 3.9. Let the measure $\mu(\cdot, \omega) \in \mathcal{B}$. Let also the process $h^{c}: \mathbb{R} \times[0, \infty) \times \Omega \rightarrow \mathbb{R}_{+}$be $\mathcal{F}_{t}$-measurable and such that $h(y, t, \omega)$ defined via

$$
h(y, t)=\left(k * h^{c}\right)(y, t)+h^{\mu}(y, t),
$$

is $\mathcal{F}_{t}$-measurable. Then, the processes $u(x, t)$ and $u^{c}(x, t)$ defined with respect to $h$ and $h^{c}$ constitute a time-monotone forward criterion.

Conversely, if $u(x, t)$ and $u^{c}(x, t)$ constitute a time-monotone forward criterion, then there exists a measure $\mu \in \mathcal{B}$ such that the associated processes $h$ and $h^{c}$ are related via (3.48).

Proof. The conditions on $\mu$ and $h^{c}$ imply that $h^{\mu}(y, t)$ and $\left(k * h^{c}\right)(y, t)$ are well defined for $(y, t, \omega) \in \mathbb{R} \times[0, \infty) \times \Omega$. Trivially, $h$ and $h^{c}$ are $\mathcal{F}_{t}$-measurable and, as argued above, they satisfy equation (3.45) a.s. Hence, according to Lemma 3.6, the associated functions $u(x, t)$ and $u^{c}(x, t)$ (defined up to affine transformations) constitute a time-monotone forward criterion.

Next, we claim that every deterministic positive solution to equation (3.45) is given by (3.48) for some measure $\mu(\cdot)$. Indeed, if $h(y, t)$ solves equation (3.45) with respect to $h^{c}$, then there exist $h^{1}$ and $h^{2}$ such that

$$
h(y, t)=h^{1}(y, t)+h^{2}(y, t),
$$

where $h^{1}$ solves equation (3.45) with the additional condition that $\lim _{t \rightarrow \infty} h(y, t)=0, y \in \mathbb{R}$, and where $h^{2}$ solves the homogeneous backward heat-equation

$$
h_{t}+\frac{1}{2} h_{y y}=0, \quad(y, t) \in \mathbb{R} \times[0, \infty) .
$$

Equation (3.45) equipped with the condition $\lim _{t \rightarrow \infty} h(y, t)=0$ admits the unique solution $h^{1}(y, t)=\left(k * h^{c}\right)(y, t)$. Moreover, according to Widder's theorem ( $\mathrm{p} 235$ in [123]), every positive 
solution to equation (3.49) is given by (3.47) for some positive Borel-measure $\mu$. This completes the proof of the claim ${ }^{3}$.

Next, assume that $u(x, t)$ and $u^{c}(x, t)$ constitute a time-monotone forward criterion. According to Lemma 3.6, the functions $h$ and $h^{c}$, both from $\mathbb{R} \times[0, \infty) \times \Omega$ to $\mathbb{R}_{+}$, then constitute an $\mathcal{F}_{t}$-measurable solution to the random PDE (3.45). Due to the claim, it follows by a path-wise argument that $h(y, t, \omega)$ is given by (3.48) for some measure $\mu(\cdot, \omega) \in \mathcal{B}$.

We stress that the requirement that $h(y, t)$ is $\mathcal{F}_{t}$-measurable is crucial. An important special case emerges when the function $h^{c}(y, t, \omega)$ is such that $\left(k * h^{c}\right)(y, t)$ is $\mathcal{F}_{t}$-measurable itself. Next, we show that the measure $\mu(\cdot, \omega)$ must then be deterministic and so, in turn, must $h^{\mu}(y, t)$.

Corollary 3.10. Let $u(x, t)$ and $u^{c}(x, t)$ constitute a time-monotone forward criterion. Then, the associated functions $h$ and $h^{c}$ are related via (3.48) for some deterministic $\mu \in \mathcal{B}$ if and only if the function $\left(k * h^{c}\right)(y, t)$ is $\mathcal{F}_{t}$-measurable.

Proof. According to Theorem 3.9, $h$ is given by (3.48) for some measure $\mu(\cdot, \omega)$. The fact that $h$ as well as $k * h^{c}$ are $\mathcal{F}_{t}$-measurable, thus, implies that so is $h^{\mu}(y, t)$. Therefore, the function

$$
h^{\mu}(y, 0)=\int_{0}^{\infty} e^{r y} \mu(d r, \omega),
$$

is deterministic and, consequently, so is the measure $\mu$. The reverse statement follows directly.

The above situation occurs, for example, when $h^{c}(y, t)$ is a deterministic function. It also occurs for $h^{c} \equiv 0$, i.e. when $h(y, t)$ represents a pure forward investment criterion. For this case, it has already been established in [8] that $h(y, t)$ corresponds to a time-monotone forward criterion if and only if $h(y, t)=h^{\mu}(y, t)$ for some deterministic measure $\mu$ (see Section 3.1.4.2). This should be contrasted to the present investment-consumption case, for which Theorem 3.9 shows that the restriction to time-monotone criteria is not enough to ensure that the corresponding measure $\mu$ is deterministic. Nevertheless, the criteria for which it is, form an important subclass. This class is further studied below (cf. Proposition 3.14). The situation of Corollary 3.10 also occurs for the case $h^{\mu}(y, t) \equiv 0$, which corresponds to the classical infinite horizon Merton criteria. This is further illustrated by the next Corollary. This result was presented also in [9]. However, there it was given without proof (cf. p 12 therein).

\footnotetext{
3 alternatively, one might rely on results by Sieveking (1978) or Jansen (2006); cf. [9].
} 
Corollary 3.11. Let $u(x, t)$ and $u^{c}(x, t)$ be a time-monotone forward criterion. Then, there exits a measure $\mu \in \mathcal{B}$ such that the associated functions $\hat{u}(y, t)$ and $\hat{u}^{c}(y, t)$ are related via

$$
\hat{u}(y, t)=\int_{t}^{\infty} \int_{-\infty}^{\infty} \hat{u}^{c}\left(y \cdot e^{\sqrt{A_{s}-A_{t}} z-\left(A_{s}-A_{t}\right) / 2}, s\right) k(z) d z d s-\int_{0}^{\infty} \frac{y^{1-r}}{r-1} e^{\frac{1}{2}\left(r-r^{2}\right) A_{t}} \mu(d r),
$$

where the first term should be understood in the sense of its path-wise limit.

Proof. According to Theorem 3.9, $h(y, t)$ is given by (3.48). That $h^{\mu}(y, t)$ gives rise to the second term in (3.50) follows from previous results on pure forward investment criteria (cf. [9, 96]). Hence, without loss of generality, let $h^{\mu}(y, t) \equiv 0$. It follows from (3.44) and the definition of $\left(k * h^{c}\right)$ that

$$
\begin{aligned}
H(y, t ; \omega) & =\int_{A_{t}(\omega)}^{\infty} \int_{-\infty}^{\infty} h^{c}\left(y+\sqrt{s-A_{t}(\omega)} z, s ; \omega\right) k(z) d z d s \\
& =\int_{t}^{\infty} \int_{-\infty}^{\infty} h^{c}\left(y+\sqrt{A_{s}(\omega)-A_{t}(\omega)} z, A_{s} ; \omega\right) k(z) d z\left|\lambda_{s}\right|^{2} d s \\
& =\int_{t}^{\infty} \int_{-\infty}^{\infty} H^{c}\left(y+\sqrt{A_{s}(\omega)-A_{t}(\omega)} z, s ; \omega\right) k(z) d z d s
\end{aligned}
$$

where the path-wise variable substitution ${ }^{4}$ could be performed due to Assumption 3.8. Hence, it follows that

$$
\begin{aligned}
H\left(-\ln y+\frac{1}{2} A_{t}, t, \omega\right) & =\int_{t}^{\infty} \int_{-\infty}^{\infty} H^{c}\left(-\ln y+\frac{1}{2} A_{t}+\sqrt{A_{s}(\omega)-A_{t}(\omega)} z, s ; \omega\right) k(z) d z d s \\
& =\int_{t}^{\infty} \int_{-\infty}^{\infty} H^{c}\left(-\ln \left(y e^{\frac{1}{2}\left(A_{s}-A_{t}\right)-\sqrt{A_{s}-A_{t}} z}\right)+\frac{1}{2} A_{s}, s ; \omega\right) k(z) d z d s
\end{aligned}
$$

which according to (3.41) implies that

$$
\hat{u}_{y}(y, t ; \omega)=\int_{t}^{\infty} \int_{-\infty}^{\infty} \hat{u}_{y}^{c}\left(y e^{\frac{1}{2}\left(A_{s}-A_{t}\right)-\sqrt{A_{s}-A_{t}} z}, s ; \omega\right) k(z) d z d s .
$$

Path-wise integration with respect to $y$ then yields

$$
\begin{aligned}
\hat{u}(y, t ; \omega) & =\int_{t}^{\infty} \int_{-\infty}^{\infty} \hat{u}^{c}\left(y e^{\frac{1}{2}\left(A_{s}-A_{t}\right)-\sqrt{A_{s}-A_{t}} z}, s ; \omega\right) \frac{e^{-\left(\sqrt{A_{s}-A_{t}}-z\right)^{2} / 2}}{\sqrt{2 \pi}} d z d s \\
& =\int_{t}^{\infty} \int_{-\infty}^{\infty} \hat{u}^{c}\left(y e^{\frac{1}{2}\left(A_{s}-A_{t}\right)-\sqrt{A_{s}-A_{t}}\left(\sqrt{A_{s}-A_{t}}+z\right)}, s ; \omega\right) k(z) d z d s \\
& =\int_{t}^{\infty} \int_{-\infty}^{\infty} \hat{u}^{c}\left(y e^{-\frac{1}{2}\left(A_{s}-A_{t}\right)-\sqrt{A_{s}-A_{t}} z}, s ; \omega\right) k(z) d z d s
\end{aligned}
$$

which completes the proof.

${ }^{4} \mathrm{As} A_{t}$ is continuous and of finite variation, a variable substitution yields

$$
\int_{A_{0}}^{A_{t}} f(s) d s=F\left(A_{t}\right)-F\left(A_{0}\right)=\int_{0}^{t} f\left(A_{s}\right) d A_{s}
$$

for any continuous function $f$ such that the above integrals are well defined. Hence, the integrals in (3.52) are not only $\mathcal{F}_{t}-$ measurable, but in fact even $\sigma\left(A_{t}\right)$-measurable. Since, according to assumption (3.43), $\lim _{t \rightarrow \infty} A_{t}=\infty$ a.s. it, thus, follows that

which, in particular, is $\mathcal{F}_{0}$-measurable.

$$
\int_{A_{0}}^{\infty} f(s) d s=\lim _{t \rightarrow \infty} \int_{A_{0}}^{A_{t}} f(s) d s=\int_{0}^{\infty} f\left(A_{s}\right) d A_{s},
$$


The above result shows that the dual value process $\hat{u}(y, t)$ is a path-wise linear combination of the dual value processes corresponding to pure forward investment criteria and pure infinite horizon Merton criteria. Indeed, the first term in formula (3.50) corresponds to a pure forward investment criterion (see, for example, [96]). Similarly, the second term of (3.50) corresponds to a classical infinite horizon Merton problem (cf. pages 121 and 131 in [74] for the log-normal case) with respect to a possibly state-dependent felicity function $u^{c}$.

Next, we make a remark on the admissible initial preferences associated with time-monotone investment-consumption criteria. Theorem 3.9 establishes that $u_{0}(x)$ corresponds to an admissible criteria if and only if the associated function $h(y, 0)$ is given by

$$
h(y, 0)=\int_{0}^{\infty} \int_{-\infty}^{\infty} k(z) h^{c}(y+\sqrt{s} z, s) d z d s+\int_{\mathbb{R}} e^{r y} \mu(d r),
$$

for some deterministic increasing function $h^{c}$ and a measure $\mu \in \mathcal{B}$. As the function $h^{c}$ is not a priori specified, it follows that the set of admissible initial conditions for the investmentconsumption case is considerably larger than the one for the pure investment case, where $h^{c} \equiv 0$. Furthermore, for the pure investment case it holds that the initial condition $h(y, 0)$ uniquely determines $h(y, t), t \geq 0$. As different functions $h^{c}$ might give rise to the same initial function $\left(k * h^{c}\right)(y, 0)$ (cf. (3.48)), this is not the case for the investment-consumption case. Note, however, that the combination of $h^{c}(y, t), t \geq 0$ and $h(y, 0), y \in \mathbb{R}$, uniquely determines $h(y, t)$, $t \geq 0$.

Next, we consider an example. We note that this is the same example as considered on page 11 in [9] (with coefficients given by $R=\frac{1}{r}$ and $Q=\frac{q}{r}$ ). We still choose to include it here as it is the most straightforward construction of a forward investment consumption criterion.

Example 3.12. Consider the functions $h$ and $h^{c}$ given by,

$$
\left\{\begin{array}{l}
h^{c}(y, t)=q e^{r y-\frac{1}{2} r^{2} t-q t} \\
h(y, t)=e^{r y-\frac{1}{2} r^{2} t-q t} .
\end{array}\right.
$$

Then the following three facts hold.

i) The deterministic functions $h$ and $h^{c}$ solve equation (3.45) and, hence, the corresponding functions $u$ and $u^{c}$ (cf. formulae (3.33) and (3.44)) constitute an investment-consumption criterion.

ii) The criterion given by $\left(u, u^{c}\right)$ corresponds to an infinite horizon Merton problem (cf. the discussion after Corollary 3.11) since it holds that

$$
h(y, t)=\left(k * h^{c}\right)(y, t)
$$


Indeed, it holds that

$$
\begin{aligned}
\left(k * h^{c}\right)(y, t) & =\int_{t}^{\infty} \int_{-\infty}^{\infty} k(z ; 1) h^{c}(y+\sqrt{s-t} z, s) d z d s \\
& =\int_{t}^{\infty} \int_{-\infty}^{\infty} k(z ; 1) q e^{r(y+\sqrt{s-t} z)-\frac{r^{2}}{2} s-q s} d z d s \\
& =q e^{r y} \int_{t}^{\infty} e^{-\frac{r^{2}}{2} s-q s} \int_{-\infty}^{\infty} k(z ; 1) e^{r \sqrt{s-t} z} d z d s \\
& =q e^{r y-\frac{r^{2}}{2} t} \int_{t}^{\infty} e^{-q s} d s=h(y, t),
\end{aligned}
$$

where was used that $\int_{-\infty}^{\infty} k(z ; 1) e^{r \sqrt{s-t} z} d z=e^{\frac{1}{2} r^{2}(s-t)}$ and $\int_{t}^{\infty} e^{-q s} d s=\frac{1}{q} e^{-q t}$.

iii) The functions $u$ and $\hat{u}$ are given by:

$$
\left\{\begin{array}{l}
u(x, t)=\frac{x^{1-1 / r}}{1-1 / r} e^{(1-r) \frac{1}{2} A_{t}-\frac{q}{r} A_{t}} \\
\hat{u}(y, t)=-\frac{1}{1-r} y^{1-r} e^{r / 2(1-r) A_{t}-q A_{t}}
\end{array}\right.
$$

and, moreover, it holds that $\hat{u}^{c}(y, t)=q\left|\lambda_{t}\right|^{2} \hat{u}(y, t)$. Indeed, according to (3.33) and (3.44) it holds that

$$
\begin{aligned}
u_{x}(x, t) & =\exp \left\{-h^{(-1)}\left(x, A_{t}\right)+\frac{1}{2} A_{t}\right\} \\
& =\exp \left\{-\frac{1}{r}\left(\ln x+\frac{r^{2}}{2} A_{t}+q A_{t}\right)+\frac{1}{2} A_{t}\right\}=x^{-1 / r} e^{(1-r) \frac{1}{2} A_{t}-\frac{q}{r} A_{t}} .
\end{aligned}
$$

Hence, the formula for $u(x, t)$ follows. Moreover, according to Lemma 3.6 we have

$$
\begin{aligned}
\hat{u}_{y}(y, t) & =-h\left(-\ln y+\frac{1}{2} A_{t}, A_{t}\right) \\
& =-\exp \left\{r\left(-\ln y+\frac{1}{2} A_{t}\right)-\frac{r^{2}}{2} A_{t}-q A_{t}\right\}=-y^{-r} e^{r / 2(1-r) A_{t}-q A_{t}} .
\end{aligned}
$$

Hence, the formula for $\hat{u}(y, t)$ follows. Finally, it follows from (3.44) that $\hat{u}^{c}(y, t)=$ $q\left|\lambda_{t}\right|^{2} \hat{u}(y, t)$.

\subsubsection{Optimal strategies, equivalent formulations and economic interpretation}

In this section, we study the optimal behaviour associated with time-monotone forward investmentconsumption criteria. First, we present explicit representations of the optimal investment strategies, wealth processes and consumption streams.

Proposition 3.13. Consider an investor with initial wealth $x$ and preferences specified by a time-monotone forward investment-consumption criterion $\left(u, u^{c}\right)$. Then, the associated optimal 
strategy, $\pi^{*}$, consumption stream, $c^{*}$, and wealth process, $X^{*}$, are given by:

$$
\left\{\begin{aligned}
\pi_{t}^{*} & =\sigma_{t}^{+} \lambda_{t} h_{y}\left(h^{(-1)}(x, 0)+M_{t}+A_{t}, A_{t}\right), \\
c_{t}^{*} & =\left|\lambda_{t}\right|^{2} h^{c}\left(h^{(-1)}(x, 0)+M_{t}+A_{t}, A_{t}\right), \\
X_{t}^{*} & =h\left(h^{(-1)}(x, 0)+M_{t}+A_{t}, A_{t}\right),
\end{aligned}\right.
$$

where $h, h^{c}, M_{t}$ and $A_{t}, t \geq 0$, are given in (3.44) and (3.31), respectively.

Proof. Assume that $X_{t}^{*}, t>0$ is defined as

$$
X_{t}^{*}=h\left(N_{t}, A_{t}\right),
$$

for some process $N_{t}, t>0$. According to (3.26) (cf. the proof of Theorem 3.1 in [9]) it, then, holds that

$$
\begin{aligned}
\pi_{t}^{*} & =-\sigma_{t}^{+} \lambda_{t} \frac{u_{x}\left(X_{t}^{*}, t\right)}{u_{x x}\left(X_{t}^{*}, t\right)} \\
& =\sigma_{t}^{+} \lambda_{t} H_{y}\left(H^{(-1)}\left(X_{t}^{*}, t\right), t\right) \\
& =\sigma_{t}^{+} \lambda_{t} h_{y}\left(h^{(-1)}\left(X_{t}^{*}, A_{t}\right), A_{t}\right)=\sigma_{t}^{+} \lambda_{t} h_{y}\left(N_{t}, A_{t}\right)
\end{aligned}
$$

and, moreover, that

$$
\begin{aligned}
c_{t}^{*} & =I^{c}\left(u_{x}\left(X_{t}^{*}, t\right), t\right) \\
& =I^{c}\left(u_{x}\left(H\left(N_{t}, t\right), t\right), t\right) \\
& =I^{c}\left(\exp \left\{-N_{t}+\frac{1}{2} A_{t}\right\}, t\right)=H^{c}\left(N_{t}, t\right)=\left|\lambda_{t}\right|^{2} h^{c}\left(N_{t}, A_{t}\right) .
\end{aligned}
$$

Hence, it follows that

$$
\begin{aligned}
d X_{t}^{*} & =\sigma_{t} \pi_{t}^{*} \cdot\left(\lambda_{t} d t+d W_{t}\right)-c_{t}^{*} d t \\
& =\lambda_{t} h_{y}\left(N_{t}, A_{t}\right) \cdot\left(\lambda_{t} d t+d W_{t}\right)-\left|\lambda_{t}\right|^{2} h^{c}\left(N_{t}, A_{t}\right) d t \\
& =\left(\left|\lambda_{t}\right|^{2} h_{y}\left(N_{t}, A_{t}\right)-\left|\lambda_{t}\right|^{2} h^{c}\left(N_{t}, A_{t}\right)\right) d t+\lambda_{t} h_{y}\left(N_{t}, A_{t}\right) \cdot d W_{t}
\end{aligned}
$$

Next, we show that the process $N_{t}, t \geq 0$, given by

$$
N_{t}=h^{(-1)}(x, 0)+M_{t}+A_{t},
$$

solves (3.55). Application of Itô's formula yields

$$
\begin{aligned}
d h\left(N_{t}, A_{t}\right) & =h_{y}\left(N_{t}, A_{t}\right) d N_{t}+\frac{1}{2} h_{y y}\left(N_{t}, A_{t}\right) d\langle N\rangle_{t}+h_{t}\left(N_{t}, A_{t}\right) d A_{t} \\
& =h_{y}\left(N_{t}, A_{t}\right)\left(d M_{t}+d A_{t}\right)+\left(\frac{1}{2} h_{y y}\left(N_{t}, A_{t}\right)+h_{t}\left(N_{t}, A_{t}\right)\right) d A_{t} \\
& =h_{y}\left(N_{t}, A_{t}\right)\left(\left|\lambda_{t}\right|^{2} d t+\lambda_{t} \cdot d W_{t}\right)-h^{c}\left(N_{t}, A_{t}\right)\left|\lambda_{t}\right|^{2} d t,
\end{aligned}
$$


which coincides with the expression given in (3.55). Therefore, $X^{*}$ is indeed given by (3.54) with the process $N$ given by (3.56). We easily conclude.

For the case when $h^{c}(y, t)$ and, consequently, $h(y, t)$ are deterministic functions (cf. Corollary 3.10 ), the above result implies that the optimal strategies are given by a compilation of the stochastic market input (represented via $A_{t}$ and $M_{t}$ ) and the deterministic functions $h$ and $h^{c}$.

Next, we present an important result for the time-monotone forward criteria for which the associated measure $\mu$ is deterministic (cf. Corollary 3.10). To give some motivation, consider an investor maximizing utility from intermediate consumption and terminal wealth with respect to some fixed terminal horizon $T>0$. It is well-known that in this case there exists a specific decomposition of the initial wealth with the property that if these parts, respectively, are invested in order to optimize utility from consumption and terminal wealth, then the resulting total investments coincide with the optimal investments for the original problem (cf. Theorem 3.7.10 in [74]). The following theorem establishes that a similar result holds also for the forward investment-consumption criteria for which $\mu$ is deterministic.

Proposition 3.14. Consider an investor with initial capital $x$ and preferences specified by a time-monotone forward investment-consumption criterion for which the associated measure $\mu$ (cf. Theorem 3.9) is deterministic. Let $\left(\pi_{t}^{*}, c_{t}^{*}\right), t \geq 0$, denote the associated optimal strategy and consumption pair (cf. Proposition 3.13).

Define $u_{1}$ with respect to $\left(k * h^{c}\right)(y, t)$ and $u_{2}$ with respect to $h^{\mu}(y, t)$ (cf. (3.33) and (3.44)), where $k * h^{c}$ and $h^{\mu}$ are given by the representation (3.48) of the function $h$. Moreover, define

$$
x_{1}=\left(k * h^{c}\right)\left(h^{(-1)}(x, 0), 0\right) .
$$

Let $\left(\pi^{1, *}, c^{1, *}\right)$ denote the optimal strategy-consumption pair with respect to initial wealth $x_{1}$ and the investment-consumption criterion $u_{1}$ and $u^{c}$. Similarly, let $\pi^{2, *}$ denote the optimal strategy with respect to initial wealth $x_{2}=x-x_{1}$ and the investment criterion $u_{2}$. Then, it holds that

$$
\left\{\begin{array}{l}
\pi_{t}^{*}=\pi_{t}^{1, *}+\pi_{t}^{2, *}, \\
c_{t}^{*}=c_{t}^{1, *}, \quad t \geq 0 .
\end{array}\right.
$$

Proof of Proposition 3.14. First, we claim that

$$
\left(k * h^{c}\right)^{(-1)}\left(x_{1}, 0\right)=h^{(-1)}(x, 0)=\left(h^{\mu}\right)^{(-1)}\left(x-x_{1}, 0\right) .
$$


Indeed, the first equality is immediate given the definition of $x_{1}$ while the second follows from the fact that

$$
\begin{aligned}
h^{(-1)}(x, t) & =\left(h^{\mu}\right)^{(-1)}\left(h^{\mu}\left(h^{(-1)}(x, t), t\right), t\right) \\
& =\left(h^{\mu}\right)^{(-1)}\left(h\left(h^{(-1)}(x, t), t\right)-\left(k * h^{c}\right)\left(h^{(-1)}(x, t), t\right), t\right) \\
& =\left(h^{\mu}\right)^{(-1)}\left(x-\left(k * h^{c}\right)\left(h^{(-1)}(x, t), t\right), t\right),
\end{aligned}
$$

for any $t \geq 0$.

Next, use of Proposition 3.13 in combination with (3.57) yields that

$$
\begin{aligned}
\pi_{t}^{1, *}+\pi_{t}^{2, *}= & \sigma_{t}^{+} \lambda_{t}\left[\left(k * h^{c}\right)_{y}\left(\left(k * h^{c}\right)^{(-1)}\left(x_{1}, 0\right)+M_{t}+A_{t}, A_{t}\right)\right. \\
& \left.\quad+h_{y}^{\mu}\left(\left(h^{\mu}\right)^{(-1)}\left(x-x_{1}, 0\right)+M_{t}+A_{t}, A_{t}\right)\right] \\
= & \sigma_{t}^{+} \lambda_{t}\left[\left(k * h^{c}\right)_{y}\left(h^{(-1)}(x, 0)+M_{t}+A_{t}, A_{t}\right)+h_{y}^{\mu}\left(h^{(-1)}(x, 0)+M_{t}+A_{t}, A_{t}\right)\right] \\
= & \sigma_{t}^{+} \lambda_{t} h_{y}\left(h^{(-1)}(x, 0)+M_{t}+A_{t}, A_{t}\right)=\pi_{t}^{*},
\end{aligned}
$$

and, moreover, that

$$
\begin{aligned}
c_{t}^{1, *} & =\left|\lambda_{t}\right|^{2} h^{c}\left(\left(k * h^{c}\right)^{(-1)}\left(x_{1}, 0\right)+M_{t}+A_{t}, A_{t}\right) \\
& =\left|\lambda_{t}\right|^{2} h^{c}\left(h^{(-1)}(x, 0)+M_{t}+A_{t}, A_{t}\right)=c_{t}^{*} .
\end{aligned}
$$

We easily conclude.

The above result implies that to invest and consume with respect to a forward criterion of this type is equivalent to split the initial wealth into two parts which are then, separately, invested according to two specific investment criteria. The first of these criteria is an infinite horizon consumption criterion specified by a felicity function and its corresponding value function; $u^{c}(x, t)$ and $u^{1}(x, t)$, respectively, in Proposition 3.14. It corresponds to an infinite horizon Merton problem where utility is obtained solely from consumption. The second criterion is a pure forward investment criterion; $u^{2}(x, t)$ in Proposition 3.14, which corresponds to the forward investment problem studied in, among others, [96]. The result therefore provides an intuitively pleasing economic interpretation of these criteria.

We stress, however, that Proposition 3.14 only holds for time-monotone forward criteria for which the associated measure $\mu$ is deterministic. In general, the functions $h^{\mu}(y, t)$ and $\left(k * h^{c}\right)(y, t)$ might not be $\mathcal{F}_{t}$-measurable although the sum of the two is. Consequently, the associated criteria can not be expressed as a deterministic compilation of an infinite horizon Merton and 
pure forward investment criterion. Rather, it constitutes a path-wise compilation of these two types of criteria. This, in turn, implies that the notion of time-monotone forward investment criteria, indeed, adds something new to the theory of optimal investment and consumption. Given that infinite horizon Merton criteria as well as forward investment criteria have been extensively studied and argued to well-describe investors preferences in various situations, the notion of forward-investment consumption criteria seems well motivated. Naturally, it would be of interest to study similar questions also for the more general class of forward criteria with non-zero volatility. 


\section{Chapter 4}

\section{Time-consistent investments under model uncertainty \& the robust forward performance criteria}

\subsection{Introduction}

In this chapter, we introduce and study the notion of robust forward performance criteria, which combines forward investment performances and ambiguity averse portfolio selection. While the former notion addresses issues of horizon and preference ambiguity, the latter deals with model uncertainty. The combination of these concepts appears natural since an investor who acknowledges her inability to specify the market model, is likely to also admit her inability to specify the preferences exactly. In the same way as standard forward criteria describe the evolution of value-processes and preferences in general, the study of robust forward criteria contribute to our understanding of the evolution of time-consistent ambiguity averse preferences. We refer to pp. 6-9 in the Introduction for a more in depth discussion of our motivation and of the relevant literature.

The main focus here is on establishing dual characterizations of the robust forward criteria. As holds for utility maximization in general, the study of the problem within the dual domain offers various advantages. In fact, this is particularly true for the case of robust preferences where the dual problem amounts to the search for an infimum whereas the primal problem features a saddle-point. To establish the duality we combine ideas developed in Schied [110] and Žitković [129]. 
This chapter is organised as follows. In Section 4.2, the market model is specified and the notion of robust forward criteria is introduced. In Section 4.3, equivalent dual characterizations of robust forward criteria are established. While the results hold for very general market models, they require rather restrictive assumptions on the penalty function. In Section 4.4, so called non-volatile robust forward criteria are considered within a Brownian filtration. The discussion is formal, the aim of which is to illustrate the flexibility of the notion and the fact that interesting preferences might be identified under additional evolutionary requirements. In particular, nonvolatile criteria are linked to a specific PDE which, formally, is discussed in further detail. In Section 4.5, an example of non-volatile logarithmic criteria is studied. The example is of interest as it illustrates that the notion accommodates criteria which produce fractional Kelly-strategies which are commonly used in practice. Despite its very specific form, the explicit solution obtained in this case also illustrates a general fact about robust forward criteria. Namely, that for each robust forward criterion, there exists a specific (standard) forward criterion in the reference market, giving rise to the same optimal behaviour. This is discussed also for more general criteria.

\subsection{The market model and definition of the criterion}

\subsubsection{The market model and notation}

We consider the same market model as set out in Section 2 in [129] and refer thereto for the details. In short, $\left(S^{0} ; S\right)=\left(S_{t}^{0}, S_{t}^{1}, \ldots, S_{t}^{d}\right)_{t \in[0, \infty)}$ is a $(d+1)$-dimensional càdlàg semi-martingale on a filtered probability space $(\Omega, \mathcal{F}, \mathbb{F}, \mathbb{P})$, where $\mathbb{F}=\left(\mathcal{F}_{t}\right)_{t \in[0, \infty)}$ satisfies the usual conditions. We let $S^{0} \equiv 1$ and assume $S$ to be locally bounded.

An $\mathbb{F}$-predictable process $\pi=\left(\pi_{t}\right)_{t \in[0, \infty)}$ is said to be an admissible portfolio if $\pi$ is $S$-integrable on $[0, T]$ for each $T>0$ and, furthermore, for any $T>0$, there exists a constant $a>0$ such that the wealth-process $X^{\pi}$ given by

$$
X_{t}^{\pi}=\int_{0}^{t} \pi_{u} d S_{u}, \quad t \geq 0
$$

is bounded from below by $-a$ for all $t \in[0, T]$, a.s. The set of all admissible portfolio processes is denoted by $\mathcal{A}$. By $\mathcal{A}_{b d}$ we denote the set of all portfolios giving rise to bounded wealth-processes; $\mathcal{A}_{b d}=\mathcal{A} \cap(-\mathcal{A})$.

For each $T>0, \mathcal{M}_{T}^{e}$ denotes the set of equivalent local martingale measures. That is to say, the set of measures $\mathbb{Q}$ on $\mathcal{F}_{T}$ such that $\left.\mathbb{Q} \sim \mathbb{P}\right|_{\mathcal{F}_{T}}$ and each component of $S$ is a $\mathbb{Q}$-local 
martingale. Similarly, $\mathcal{M}_{T}^{a}$ denotes the set of absolutely continuous local martingale measures. The corresponding sets of density processes are denoted by $\mathcal{Z}_{T}^{e}$ and $\mathcal{Z}_{T}^{a}$, respectively:

$$
\mathcal{Z}_{T}^{e}=\left\{Z=\frac{\mathrm{d} \mathbb{Q}}{\left.\mathrm{d} \mathbb{P}\right|_{\mathcal{F}_{T}}}: \mathbb{Q} \in \mathcal{M}_{T}^{e}\right\},
$$

and similarly for $\mathcal{Z}_{T}^{a}$. We assume that the set $\mathcal{M}_{T}^{e}$ is non-empty for each $T>0$ which is equivalent to absence of arbitrage (FLVR) on finite horizons (cf. [129]). Note that

$$
\mathcal{M}_{T_{1}}^{e}=\left\{\left.\mathbb{Q}\right|_{\mathcal{F}_{T_{1}}}: \mathbb{Q} \in \mathcal{M}_{T_{2}}^{e}\right\}, \quad \text { for all } 0 \leq T_{1} \leq T_{2} .
$$

However, there need not exist a set $\mathcal{M}^{e}$ of probability measures equivalent to $\mathbb{P}$ such that $\mathcal{M}_{T}^{e}=\left\{\left.\mathbb{Q}\right|_{\mathcal{F}_{T}}: \mathbb{Q} \in \mathcal{M}^{e}\right\}$, for $T>0$.

As argued in [129], the condition of NFLVR on finite horizons implies that, for each $\mathbb{Q} \in \mathcal{M}_{T}^{e}$, the density process $Z_{t}^{\mathbb{Q}}=\mathrm{E}\left[\frac{\mathrm{dQ}}{\left.\mathrm{d} \mathbb{P}\right|_{\mathcal{F}_{T}}} \mid \mathcal{F}_{t}\right], t \in[0, T]$, might be extended to a strictly positive martingale $\left(Z_{t}\right)_{t \in[0, \infty)}$ such that $Z_{0}=1$ and $Z S$ is a local martingale. The set of all such processes $Z$ will be denoted by $\mathcal{Z}^{e}$. In particular, NFLVR on finite horizons holds if and only if $\mathcal{Z}^{e}$ is non-empty. Furthermore, if the condition of strict positivity is replace by the one of non-negativity, the obtained family is denoted by $\mathcal{Z}^{a}$. For any $\mathbb{Q} \ll \mathbb{P}$, we use the notation $Z_{t, T}^{\mathbb{Q}}:=Z_{T}^{\mathbb{Q}} / Z_{t}^{\mathbb{Q}}$, with the convention that $Z_{t, T}^{\mathbb{Q}} \equiv 1$ on $\left\{Z_{t}^{\mathbb{Q}}=0\right\}$.

\subsubsection{Robust forward performance criteria}

Robust forward performance criteria will combine two elements: a utility random field $U$ and a penalty function $\gamma . U(\omega, x, t)$ expresses how the agent feels about having wealth $x$ at time $t$, given what has happen so far, i.e. for a fixed $\omega$. The agent, who is uncertain about the "true model", forms a view about the relative plausibility of different probability measures to best describe the dynamics of financial assets. This is reflected in $\gamma_{t, T}(\mathbb{Q})(\omega)$ which gives the relative weighting of measure $\mathbb{Q}$ on $\mathcal{F}_{T}$. One may expect $\gamma_{t, T}(\cdot)(\omega)$ to have a global minimum which describes agent's estimation of the true model. This will be the case in the example considered in Section 4.5. In that example, we will also see that the randomness of $U(\cdot, x, t)$ is expressed through the evolution of agent's most likely probability measure.

Both $U$ and $\gamma$ are combined in making investment decisions. More precisely, to asses an investment strategy the agent considers the expected value of utility $U$ of her future wealth. When comparing expectations under different measures, the agent takes into account their relative weighting specified through $\gamma$. This leads to the value function defined in (4.2) below. 
Definition 4.1. A random field is a mapping $U: \Omega \times \mathbb{R} \times[0, \infty) \rightarrow \mathbb{R}$ which is measurable with respect to the product of the optional $\sigma$-algebra on $\Omega \times[0, \infty)$ and $\mathcal{B}(\mathbb{R})$. A utility random field is a random field which satisfies the following conditions:

i) For all $t \in[0, \infty)$, the mapping $x \rightarrow U(\omega, x, t)$ is $\mathbb{P}$-a.s. a strictly concave and increasing $C^{1}(\mathbb{R})$-function which satisfies the Inada conditions

$$
\lim _{x \rightarrow-\infty} \frac{\partial}{\partial x} U(\omega, x, t)=\infty, \quad \lim _{x \rightarrow \infty} \frac{\partial}{\partial x} U(\omega, x, t)=0
$$

ii) For all $x \in \mathbb{R}$, the mapping $t \rightarrow U(\omega, x, t)$ is càdlàg on $[0, \infty)$;

iii) For each $x \in \mathbb{R}$ and $T \in[0, \infty), U(\cdot, x, T) \in L^{1}\left(\mathcal{F}_{T}\right)$.

In what follows, we suppress $\omega$ from the notation and simply write $U(x, t)$.

Definition 4.2. For given $t \leq T<\infty$, a mapping $\gamma_{t, T}: \Omega \times\left\{\mathbb{Q}:\left.\left.\mathbb{Q}\right|_{\mathcal{F}_{T}} \sim \mathbb{P}\right|_{\mathcal{F}_{T}}\right\} \rightarrow \mathbb{R}_{+} \cup\{\infty\}$, is called a penalty function if

i) $\gamma_{t, T}$ is $\mathcal{F}_{t}$-measurable;

ii) $\mathbb{Q} \rightarrow \gamma_{t, T}(\mathbb{Q})$ is convex a.s;

iii) for $\kappa \in L_{+}^{\infty}\left(\mathcal{F}_{t}\right), \mathbb{Q} \rightarrow \mathrm{E}\left[\kappa \gamma_{t, T}(\mathbb{Q})\right]$ is weakly lower semi-continuous on $\left\{\mathbb{Q}:\left.\mathbb{Q} \sim \mathbb{P}\right|_{\mathcal{F}_{T}}\right\}$.

Moreover, for a given utility random field $U(x, t)$, we say that $\left(\gamma_{t, T}\right), 0 \leq t \leq T<\infty$, is an admissible family of penalty functions if for all $T>0$ and $x \in \mathbb{R}, \mathrm{E}^{\mathbb{Q}}[U(x, T)]$ is well defined in $\mathbb{R} \cup\{\infty\}$ for all $\mathbb{Q} \in \mathcal{Q}_{t, T}, t \leq T$, where $\mathcal{Q}_{t, T}$ denotes the following set of measures on $\mathcal{F}_{T}$ :

$$
\mathcal{Q}_{t, T}:=\left\{\mathbb{Q}:\left.\mathbb{Q} \sim \mathbb{P}\right|_{\mathcal{F}_{T}} \text { and } \gamma_{t, T}(\mathbb{Q})<\infty \text { a.s. }\right\}
$$

In the above definition the set of feasible measures $\mathcal{Q}_{t, T}$ considered at time $t$ when investing over $[t, T]$ may depend on $t, T$ but is not random. It is a somewhat arbitrary choice and both larger and smaller sets could be used, e.g. the set of measures $\mathbb{Q}$ with $\gamma_{t, T}(\mathbb{Q})(\omega)<\infty$ or the set of measures $\mathbb{Q}$ with $\mathrm{E}\left[\gamma_{t, T}(\mathbb{Q})\right]<\infty$. However, for many natural penalty functions, the three sets lead to the same value function, see Section 4.3 .2 below. Note also that we did not impose any regularity assumptions on $\gamma_{t, T}(\mathbb{Q})$ in time variables. These are not necessary for the abstract results in Section 4.3 and will be introduced later when they appear naturally, see Assumption 4.10 .

Given the above definitions of utility random fields and associated families of penalty functions, robust forward criteria are defined as pairs which are time-consistent: 
Definition 4.3. Let $U$ be a utility random field and $\gamma$ an admissible family of penalty functions. Then, the value field associated with $U$ and $\gamma$ is a family of mappings $\quad\{u(\cdot ; t, T): 0 \leq t \leq T<$ $\infty\}$, with $u(\cdot ; t, T): L^{\infty}\left(\mathcal{F}_{t}\right) \rightarrow L^{0}\left(\mathcal{F}_{t} ; \mathbb{R} \cup\{\infty\}\right)$ given by

$$
u(\xi ; t, T):=\underset{\pi \in \mathcal{A}_{b d}}{\operatorname{ess} \sup } \operatorname{esinf}_{\mathbb{Q} \in \mathcal{Q}_{t, T}}\left\{\mathrm{E}^{\mathbb{Q}}\left[U\left(\xi+\int_{t}^{T} \pi_{s} d S_{s}, T\right) \mid \mathcal{F}_{t}\right]+\gamma_{t, T}(\mathbb{Q})\right\}, \quad \text { for } \xi \in L^{\infty}\left(\mathcal{F}_{t}\right) .
$$

We say that the combination of a utility random field and a family of penalty functions is a robust forward performance criterion (or self-generating) if

$$
U(\xi, t)=u(\xi ; t, T), \text { a.s. }
$$

for all $0 \leq t \leq T<\infty$ and all $\xi \in L^{\infty}\left(\mathcal{F}_{t}\right)$.

With the assumptions on $U$ and $\gamma$, the conditional expectations in (4.2) are well-defined (extended valued) random variables (see e.g. Prop 18.1.5 in [113] or p. 113 in [60] for the definition of conditional expectations of quasi-integrable random variables). As all $\mathbb{Q} \in \mathcal{Q}_{t, T}$ are equivalent to $\mathbb{P}$, it also holds for each $\pi \in \mathcal{A}_{b d}$ that the essential infimum is well-defined (extended-valued) with respect to the reference measure $\mathbb{P}$.

The supremum in the above definition is taken over bounded wealth processes and, for most utility fields, may not be attained. In particular, the definition of robust forward criteria does not require it to be. However, as we now argue, this is natural and not restrictive. Indeed, the utility field defined on the entire real line does not possess any singularities (cf. the non-singularity assumption below). The value field defined with respect to a more general (but feasible) set of admissible strategies would coincide with the one defined with respect to bounded strategies. The above definition would still apply, since the notion of robust forward criteria is a consistency requirement placed on the preferences themselves, without a reference to an optimal strategy. In consequence, for utility fields defined on the entire real line, robust forward criteria defined above may be studied and characterized without exactly specifying the domain of optimization.

Within the present framework where the preferences are finite on the entire real line and in addition to that stochastic, the exact specification of a feasible set of admissible, not necessarily bounded, strategies is highly non-trivial (cf. [109]). It is therefore convenient to focus on bounded wealth processes. Note that in doing so we follow the approach in [129] rather than the original definition (cf. [96] and [97]) which required the optimum to be attained ${ }^{1}$. In Section 4.5 we consider a robust forward criterion of logarithmic type, defined on the positive line only. There, we allow for all non-negative wealth processes and establish existence of an optimizer.

\footnotetext{
${ }^{1}$ For further remarks on the flexibility obtained with this approach, we refer to Remark 3.8 in [129].
} 


\subsection{Dual characterizations of robust performance criteria}

Dual methods are well known to be useful for the study of optimal investment problems. For the standard utility maximization problem, the aim of introducing and studying the dual problem is to prove existence of and characterize the optimal strategy for the primal problem. Here, the focus is on the evolution of the preferences themselves rather than the optimal strategy. Nevertheless, there are clear benefits in passing to the dual domain: the dual problem amounts to a search for an infimum whereas the primal problem features a saddle-point. In consequence, the robust forward criteria are easier to characterize in the dual rather than the primal domain. The aim of this section is to establish such equivalent characterizations. We adopt a convenient set of assumptions with possible extensions discussed in Remarks 4.8-4.9 below.

We develop duality theory for utility random fields in Definition 4.1 which are defined on the whole real line. The reason is twofold. First, we complement the results in the literature ${ }^{2}$. Our results are related to the ones established in [110], who considered utilities defined only on the positive half-line. Second, as argued above, considering utilities finite on the entire real line simplifies certain aspects of the duality theory. While analogous results could be pursued for utilities defined on the half-line, it would imply additional technicalities and we leave it for future research (cf. Remark 3.2 in [129]).

For a given utility random field $U$, the associated dual random field $V: \Omega \times[0, \infty) \times(0, \infty) \rightarrow \mathbb{R}$, is given by

$$
V(y, t)=\sup _{x \in \mathbb{R}}(U(x, t)-x y) \quad \text { for } t \geq 0, y \geq 0 .
$$

The dual value field and the notion of self-generation in the dual domain are then naturally defined as follows:

Definition 4.4. For $y>0$ and $0 \leq t<T<\infty$ the dual value field $v(\cdot ; t, T): L_{+}^{0}\left(\mathcal{F}_{t}\right) \rightarrow$ $L^{0}\left(\mathcal{F}_{t} ; \mathbb{R} \cup\{\infty\}\right)$, is given by

$$
v(\eta ; t, T):=\underset{\mathbb{Q} \in \mathcal{Q}_{t, T}}{\operatorname{ess} \inf } \operatorname{sinf}_{Z \in \mathcal{Z}_{T}^{a}}\left\{\mathrm{E}^{\mathbb{Q}}\left[V\left(\eta Z_{t, T} / Z_{t, T}^{\mathbb{Q}}, T\right) \mid \mathcal{F}_{t}\right]+\gamma_{t, T}(\mathbb{Q})\right\} .
$$

The combination of a dual random field $V$ and a family of penalty functions $\gamma$ is said to be self-generating if

$$
V(\eta, t)=v(\eta ; t, T), \text { a.s. }
$$

for all $0 \leq t \leq T<\infty$ and all $\eta \in L_{+}^{0}\left(\mathcal{F}_{t}\right)$.

\footnotetext{
${ }^{2}$ The authors in [47] also consider risk and ambiguity averse portfolio optimization with utility functions finite on the entire real line. In particular, they also exploit the fact that this simplifies things in certain ways in order to obtain their results which, however, are different from ours. The author thanks the examiners of this DPhil thesis for this valuable reference; see also Remark 4.8 below.
} 
In order to establish the results in this section, the following technical assumption is needed.

Assumption 4.5. For each $T>0$ and $t \leq T$, the set $\mathcal{Q}_{t, T}$ is convex and weakly compact and the set $\left\{Z U^{-}(x, T): Z \in \mathcal{Q}_{t, T}\right\}$ is UI for all $x \in \mathbb{R}$. Furthermore, for $T>0, t \leq T, \kappa \in L_{+}^{\infty}\left(\mathcal{F}_{t}\right)$ and $\mathbb{Q} \in \mathcal{Q}_{t, T}$ such that $\kappa Z_{t, T}^{\mathbb{Q}} U(x, T) \in L^{1}, x \in \mathbb{R}$, it holds that

$$
\tilde{U}(x, T):=\mathbb{1}_{\kappa=0} U(x, T)+\mathbb{1}_{\kappa>0} Z_{t, T}^{\mathbb{Q}} U(x, T), \quad x \in \mathbb{R},
$$

satisfies the non-singularity Assumption 3.3 in [129].

The above assumption implies that $U(x, t)$ itself satisfies the non-singularity assumption. For further discussion of this concept, we refer to Remark 3.4 in [129]. Given that $\mathcal{Q}_{t, T}$ is weakly compact, a sufficient condition for Assumption 4.5 to hold, is that $U(x, t)$ is $(x, \omega)$-uniformly bounded from below by a deterministic utility function. Then it also trivially holds that any family of penalty functions is admissible. Note also that, due to convexity, weak compactness of $\mathcal{Q}_{t, T}$ is equivalent to closedness in $L^{0}$, cf. Lemma 3.2 in [111].

Next, the first main result, namely the conjugacy relations between the functions $u(x ; t, T)$ and $v(y ; t, T)$ is presented. Note that even for $t=0$ Theorem 4.6 differs from Theorem 2.4 in [110] in that the utility function is defined on the entire real line and possibly stochastic. Moreover, we do not impose any finiteness assumptions on the involved value-fields.

Theorem 4.6. Let $U(x, t), t \geq 0$, be a utility random field, $\left(\gamma_{t, T}\right)$ an admissible family of penalty functions and $V$ the associated dual random field. Then, under Assumption 4.5, the primal and dual value fields satisfy the following relations:

$$
\begin{aligned}
& u(\xi ; t, T)=\underset{\eta \in L_{+}^{0}\left(\mathcal{F}_{t}\right)}{\operatorname{essinf}}(v(\eta ; t, T)+\xi \eta) \quad \text { a.s. and } \\
& v(\eta ; t, T)=\operatorname{essipp}_{\xi \in L^{\infty}\left(\mathcal{F}_{t}\right)}(u(\xi ; t, T)-\xi \eta) \quad \text { a.s. }
\end{aligned}
$$

for all $0 \leq t \leq T<\infty, \xi \in L^{\infty}\left(\mathcal{F}_{t}\right)$ and $\eta \in L_{+}^{0}\left(\mathcal{F}_{t}\right)$.

The proof is given in the Appendix and is based on combining ideas introduced in [110] and [129], respectively. In the former, duality results for the robust utility maximization problem with variational preferences were established. In the latter, in a setting similar to ours, conditional conjugacy relations were established for the non-robust case. Specifically, by taking expectations, the conditional case is reduced to an $\mathcal{F}_{0}$-measurable conjugacy relation. For the latter, equality is proven using arguments similar to the ones in [110]. However, here, the arguments are applied to the non-robust duality results as formulated by Zitkovic in [129] instead of the duality results in Kramkov and Schachermayer [79], upon which [110] relies. 
The following characterization follows directly from the above result.

Corollary 4.7. Under the assumptions of Theorem 4.6, the combination of a utility random field $U$ and a family of penalty functions $\gamma$ is self generating if and only if the combination of the dual random field $V$ and the family of penalty functions $\gamma$ is self-generating.

The above results use the set of measures $\mathcal{Q}_{t, T}$ defined in (4.1) and assumed to be weakly compact. We end this section with some remarks on possible further extensions in the definition and assumptions imposed on $\mathcal{Q}_{t, T}$.

Remark 4.8. It follows immediately that under the assumption that $\mathcal{Q}_{t, T}^{a}$ is weakly compact, where $\mathcal{Q}_{t, T}^{a}$ is the set of absolutely continuous measures for which the penalty is finite a.s., Theorem 4.6 holds with the set $\mathcal{Q}_{t, T}$ replaced by $\mathcal{Q}_{t, T}^{a}$ in the definition of $u(\cdot ; t, T)$ but with the dual field still defined as above with respect to the equivalent measures. This assumption holds, in particular, for all penalty functions associated with coherent risk measures continuous from below, see Section 4.3.2 below. In order to use $\mathcal{Q}_{t, T}^{a}$ in the definition of $v(\cdot ; t, T)$ one would need to extend the definition of $Z^{\mathbb{Q}} V\left(\eta / Z^{\mathbb{Q}}\right)$ to the null-sets of $\mathbb{Q}$ in a suitable way (preserving lower semicontinuity). For the case of utility functions defined on $\mathbb{R}_{+}$, this was done in [110]. We expect this to be possible also for the present case. Indeed, in [47] the risk and ambiguity averse problem is studied for utility functions defined on the entire real line when allowing for absolutely continuous measures. Similar arguments are likely to apply also in our case and is the focus of future research. We also leave for future research to investigate whether the projection results obtained in [47] could be extended to the variational setting.

Remark 4.9. In [110], for the case of positive wealth processes and a fixed time horizon, similar conjugacy relations to (4.6)-(4.7) were established without the compactness assumption on $\mathcal{Q}_{t, T}$. The proof exploited instead weak compactness of the level-sets $\mathcal{Q}(c):=\left\{\mathbb{Q} \ll \mathbb{P}: \gamma_{0, T}(\mathbb{Q}) \leq c\right\}$. Specifically, since $U\left(\varepsilon+X_{T}\right), X_{T} \geq 0$, is uniformly bounded from below for that case, the infimum in

$$
\sup _{\pi} \inf _{\mathbb{Q} \ll \mathbb{P}}\left\{\mathrm{E}^{\mathbb{Q}}\left[U\left(\varepsilon+X_{T}^{\pi}\right)\right]+\gamma_{0, T}(\mathbb{Q})\right\}
$$

can be replaced by the infimum over some (weakly compact) level set $\mathcal{Q}(c), c>0$. After application of a minmax theorem, the result is then obtained by letting $\varepsilon$ tend to zero. Since we consider $U: \mathbb{R} \rightarrow \mathbb{R}$, things are more involved. Even for $t=0, U(x, T)$ deterministic and $\pi_{n}$ an optimizing sequence, it is not clear whether $\mathrm{E}\left[U\left(X_{T}^{\pi_{n}}, T\right)\right]$ is bounded from below. To address such issues one might have to adopt the more elaborate setup considered in [109] where the existence of an optimizer for utility functions defined on the entire real line is proven. We leave these problems for future research. 


\subsubsection{Further results on robust self-generation}

The definition of robust forward criteria requires the combined criterion consisting of $U(x, t)$ and $\gamma(\cdot)$ to be time-consistent (cf. Definition 4.3). In this section we explore the consequences of further assuming that the family of penalty functions alone are time-consistent. This in fact is rather natural, see Section 4.3.2.

Assumption 4.10. For any $T>0$ and $\mathbb{Q} \sim \mathbb{P}$ on $\mathcal{F}_{T}$, the family of penalty functions $\left(\gamma_{t, T}\right)$ is càdlàg in $t \leq T, \gamma_{t, t} \equiv 0$ and

$$
\gamma_{s, T}(\mathbb{Q})=\gamma_{s, t}(\mathbb{Q})+\mathrm{E}^{\mathbb{Q}}\left[\gamma_{t, T}(\mathbb{Q}) \mid \mathcal{F}_{s}\right], \quad s \leq t \leq T
$$

Moreover, $\tilde{\mathcal{Q}}_{s, T}=\mathcal{Q}_{s, T}$, where

$$
\tilde{\mathcal{Q}}_{s, T}:=\left\{\left.\mathbb{Q} \sim \mathbb{P}\right|_{\mathcal{F}_{T}}: Z_{T}^{\mathbb{Q}}=Z_{t}^{\mathbb{Q}_{0}} Z_{t, T}^{\mathbb{Q}_{1}}, \mathbb{Q}_{0} \in \mathcal{Q}_{s, t}, \mathbb{Q}_{1} \in \mathcal{Q}_{t, T}, s \leq t \leq T\right\}
$$

We note that the property of stability under pasting (4.9) in the above assumption is not implied by (4.8). For remarks on the relation of the above properties to penalty functions associated with risk measures, see Section 4.3 .2 below.

\subsubsection{Time-consistency of the value-field}

The additional structure resulting from Assumption 4.10 allows us to consider the question of whether the value-field itself is self-generating. That is to say, whether the value field associated with a given utility random field $U(x, t)$ and fixed horizon $T>0$, satisfies

$$
\left.u(x ; s, T)=\underset{\pi \in \mathcal{A}_{b d}}{\operatorname{ess} \operatorname{esp} \inf } \underset{\mathcal{Q}_{s, t}}{\operatorname{es}}\left[u\left(x+\int_{s}^{t} \pi_{u} d S_{u} ; t, T\right) \mid \mathcal{F}_{s}\right]+\gamma_{s, t}(\mathbb{Q})\right\}, \quad s \leq t \leq T .
$$

For the case $U(x, T)=U(x)$ for some deterministic function $U(x)$, this amounts to the question of whether the value-process of the robust utility-maximization problem (as defined in, among others, [110]) satisfies the dynamic programming principle. For the non-robust case, this is wellknown to be the case (cf. the martingale optimality principle in [37]). We verify now that under suitable assumptions on the penalty function, this holds also for the robust case. We start with a Lemma in the dual domain.

Lemma 4.11. Let $V$ be a dual random field and $\gamma$ an admissible family of penalty functions such that either Assumption 4.10 holds or $(4.8)$ holds and $v^{-}(\zeta ; t, T) \in L^{1}\left(\mathcal{F}_{t} ; \mathbb{Q}\right)$ for all $\zeta \in L^{0}\left(\mathcal{F}_{t}\right)$ and $\mathbb{Q} \in \tilde{\mathcal{Q}}_{0, T}, t \leq T$. Moreover, assume that for each $t \leq T$, the infimum in (4.4) is attained for some $\mathbb{Q} \in \mathcal{Q}_{t, T}$ and $Z_{T} \in \mathcal{Z}_{T}^{a}$. Then, the dual value field $v(\cdot ; t, T)$ is self-generating on $[0, T]$. 
Remark 4.12. Note that for penalty functions for which $\mathcal{Q}_{t, T}$ is weakly compact, existence of a minimizer seems natural. Indeed, as for the case of a fixed measure (cf. [129]), the dual problem is likely to admit a solution, even though the primal problem may not (due to the restriction to bounded strategies). The fact that the optimizer's second component is in $\mathcal{M}_{T}^{a}$ (as opposed to a larger set of finitely additive measures) is due to the utility function being finite on the entire real line; see [129] and also [109]. Without the compactness of $\mathcal{Q}_{t, T}$ to obtain existence of a minimiser above, it seems one should extend the setting from equivalent measures and define the dual problem for absolutely continuous measures. Then the compactness of the level-sets could be exploited in order to obtain existence above (cf. Remark 4.9). We leave the above challenges for future research.

Corollary 4.7 and Lemma 4.11 combined immediately give us time-consistency of the primal value function:

Proposition 4.13. Let $U(x, t)$ be a utility random field and $\gamma$ an admissible family of penalty functions. Suppose Assumptions 4.5 and 4.10 hold and that for each $t \leq T$ the infimum in (4.4) is attained for some $\mathbb{Q} \in \mathcal{Q}_{t, T}$ and $Z_{T} \in \mathcal{Z}_{T}^{a}$. Then, for each $T>0$, the primal value field $u(\cdot ; t, T)$ is self-generating for $t \leq T$.

The definition of standard forward criteria (cf. [96]) was motivated by the fact that the valueprocess in a standard utility maximization problem satisfies the dynamic programming (martingale optimality) principle. Proposition 4.13 shows that a similar property holds for certain ambiguity averse investment criteria, justifying further our definition of robust forward criteria. We note however that the value-field associated with a general penalty function may not be time-consistent (see [110] for counter-examples). Hence, while standard forward criteria might be viewed as direct extensions of value-functions associated with stochastic utility functions, Definition 4.3 enforces an additional restriction on robust forward criteria. The condition of time-consistency is necessary since the investor's horizon is not a priori known.

\subsubsection{Submartingale property of the dual field}

We establish in this section a characterization of robust forward criteria in terms of certain "weighted submartingale" property of the dual field. This will be then used to derive equation which might be used to find examples of robust forward criteria $(U, \gamma)$. As before, we first establish a Lemma in the dual domain and then deduce consequences for the primal field.

Lemma 4.14. Consider a dual random field $V$ related via (4.3) to some utility random field and an admissible family of penalty functions $\left(\gamma_{t, T}\right)$ satisfying (4.8). Assume, furthermore, that for 
each $\eta \in L_{+}^{0}$ and $t \leq T<\infty$ such that $v(\eta ; t, T)<\infty$ a.s., the dual value-function (4.4) attains its infimum for some $\mathbb{Q} \in \mathcal{Q}_{t, T}$ and $Z \in \mathcal{Z}_{T}^{a}$.

Then the following two statements are equivalent.

i) $V(y, t)$ and $\gamma$ are self-generating.

ii) For each $y>0$ and for all $t \leq T<\infty$,

$$
V\left(y Z_{t} / Z_{t}^{\mathbb{Q}}, t\right) \leq \mathrm{E}^{\mathbb{Q}}\left[V\left(y Z_{T} / Z_{T}^{\mathbb{Q}}, T\right) \mid \mathcal{F}_{t}\right]+\gamma_{t, T}(\mathbb{Q}),
$$

for all $\mathbb{Q} \in \mathcal{Q}_{t, T}$ and $Z \in \mathcal{Z}_{T}^{a}$. Moreover, for each $\bar{T}>0$, there exists $\overline{\mathbb{Q}} \in \mathcal{Q}_{0, \bar{T}}$ and $\bar{Z} \in \mathcal{Z}_{T}^{a}$, such that (4.11) holds with equality for all $t \leq T \leq \bar{T}$.

Furthermore, if either a) the set $\mathcal{Q}_{0, T}=\tilde{\mathcal{Q}}_{0, T}, T>0$; or b) for any $T>0$ and all $\zeta \in L^{0}\left(\mathcal{F}_{T}\right)$, $V^{-}(\zeta, T) \in L^{1}\left(\mathcal{F}_{T} ; \mathbb{Q}\right)$ for all $\mathbb{Q} \in \tilde{\mathcal{Q}}_{0, T} ;$ then $\left.i\right)$ and ii) are equivalent to the following condition:

iii) For each $y>0$ and for all $t \leq T<\infty$, (4.11) holds for all $\mathbb{Q} \in \mathcal{Q}_{t, T}$ and $Z \in \mathcal{Z}_{T}^{a}$. Moreover, there exists $Z \in \mathcal{Z}^{a}$ and a sequence $\left(\mathbb{Q}_{T^{i}}\right), i=1, \ldots$, such that $\mathbb{Q}_{T^{i}}=\left.\mathbb{Q}_{T^{i+1}}\right|_{\mathcal{F}_{T^{i}}}$ and $\mathbb{Q}_{T}:=\left.\mathbb{Q}_{T^{i}}\right|_{\mathcal{F}_{T}} \in \mathcal{Q}_{0, T}, T \leq T^{i}$, for which (4.11) holds with equality for the choice of $\mathbb{Q}_{T}$ and $Z_{T}$, this for all $0<t<T<\infty$.

A sufficient condition for assumption b) to hold, is that $U(x, T) \in L^{1}\left(\mathcal{F}_{T}, \mathbb{Q}\right)$ for all $\mathbb{Q} \in \tilde{\mathcal{Q}}_{0, T}$, $T>0$ (cf. Proposition 3.9 in [129]) and for the existence assumption see Remark 4.12 above. Finally, Corollary 4.7 and Lemma 4.14 together yield a characterization of time--consistency in the primal domain:

Proposition 4.15. Let $U(x, t)$ be a utility random field and $\gamma$ an admissible family of penalty functions such that Assumptions 4.5 and 4.10 hold. Assume, furthermore, that for each $\eta \in L_{+}^{0}$ and $t \leq T<\infty$ such that $v(\eta ; t, T)<\infty$ a.s., the infimum in (4.4) is attained for some $\mathbb{Q} \in \mathcal{Q}_{t, T}$ and $Z \in \mathcal{Z}_{T}^{a}$. Then $U(x, t)$ and $\gamma$ are self-generating if and only if property iii) of Lemma 4.14 holds.

\subsubsection{Penalty functions associated with risk measures}

Recall that preferences specification akin to (4.2) is motivated by results in economics. Axiomatic approach to ambiguity averse choices under uncertainty led to numerical representation in terms of concave utility functionals with the penalty function appearing naturally from the robust representation of convex risk measures, see Gilboa and Schmeidler [54], Maccheroni et al. [87] and Föllmer, Schied and Weber [46] for an overview. We summarize now some facts about such penalty functions and relate them to our assumptions. 


\section{Definition and general remarks}

Let $\rho_{t, T}$ be a conditional convex risk measure and $\gamma_{t, T}$ its associated minimal penalty function (which we assume to be bounded from below), given by

$$
\gamma_{t, T}(\mathbb{Q}):=\operatorname{ess}_{X \in L^{\infty}\left(\mathcal{F}_{T}\right)}\left(\mathrm{E}^{\mathbb{Q}}\left[-X \mid \mathcal{F}_{t}\right]-\rho_{t, T}(X)\right)
$$

for $\left.\mathbb{Q} \ll \mathbb{P}\right|_{\mathcal{F}_{T}}$. Note that it then holds that

$$
\rho_{t, T}(X)=\operatorname{ess}_{\substack{\left.\mathbb{Q} \ll \mathbb{P}\right|_{\mathcal{F}_{T}}: \\\left.\mathbb{Q}\right|_{\mathcal{F}_{t}}=\left.\mathbb{P}\right|_{\mathcal{F}_{t}}}}\left(\mathrm{E}^{\mathbb{Q}}\left[-X \mid \mathcal{F}_{t}\right]-\gamma_{t, T}(\mathbb{Q})\right)
$$

for $X \in L^{\infty}\left(\mathcal{F}_{T}\right)$ (we refer e.g. to $[11,34]$ for details on conditional risk-measures). Within the context of ambiguity averse portfolio optimization, it is common to restrict to risk-measures $\rho_{t, T}$ which are continuous from below, i.e. for $Y^{n} \in L^{\infty}$ such that $Y_{n} \nearrow Y$ a.s. with $Y \in L^{\infty}$, $\rho_{t, T}\left(Y_{n}\right) \rightarrow \rho_{t, T}(Y)$ a.s., and, furthermore, that are sensitive in that $\mathbb{P}\left(\mathrm{E}\left[\rho_{t, T}(-\varepsilon A)\right]>0\right)>0$, for all $\varepsilon>0$ and $A \in \mathcal{F}_{T}$ such that $\mathbb{P}(A)>0$. These properties, respectively, render the associated level sets $\left\{\mathbb{Q} \ll \mathbb{P}: \gamma_{0, T}(\mathbb{Q})<c\right\}, c>0$, weakly compact and $\mathcal{Q}_{e}$ non-empty (cf. Lemma 4.1 in [110] and Remark 4.9 above).

We note that in (4.12)-(4.13) $X \in L^{\infty}$ while in our case $U\left(X_{T}^{\pi}, T\right)$ is only in $L^{1}$. However this is not an issue and we do not have to restrict $\gamma$ in line with extensions of the risk-measure theory to $L^{p}$-spaces, $p=[1, \infty)$, (see $[42,70]$ and, for the conditional case, $[1,41]$ ). Here, in analogy to Schied [110], it is sufficient to impose (weaker) joint integrability conditions on $U(x, t)$ and $\gamma$ rendering the value function $u(\cdot ; t, T)$ well-defined (cf. Definition 4.2).

A penalty function $\gamma_{t, T}$ in (4.12) associated with a risk-measure satisfies properties i) - iii) of Definition $4.2^{3}$. However, in general, it will not satisfy the weak compactness assumptions used above (cf. Assumption 4.5). To illustrate this, note that for this type of penalty functions, it is natural to restrict the set $\mathcal{Q}_{t, T}$ in (4.1) to its subset (cf. e.g. Theorem 1.4 in [2]):

$$
\left\{\left.\mathbb{Q} \sim \mathbb{P}\right|_{\mathcal{F}_{T}}: \mathrm{E}^{\mathbb{Q}}\left[\gamma_{t, T}(\mathbb{Q})\right]<\infty\right\}
$$

${ }^{3}$ Indeed, the penalty function $\gamma_{t, T}$ satisfies (cf. Remark 2.7 in [43])

$$
\gamma_{t, T}(Z)=\underset{Y \in \mathcal{A}_{t, T}}{\operatorname{ess} \sup } \mathrm{E}\left[-Z Y \mid \mathcal{F}_{t}\right]
$$

where $\mathcal{A}_{t, T}$ is the acceptance set defined by $\mathcal{A}_{t, T}:=\left\{Y \in L^{\infty}\left(\mathcal{F}_{T}\right) \mid \rho_{t, T}(Y) \leq 0\right\}$. As argued in the proof of Lemma 2.6 in [43], the set $\left\{\mathrm{E}\left[-Z Y \mid \mathcal{F}_{t}\right] \mid Y \in \mathcal{A}_{t, T}\right\}$ is directed upwards for any $Z \in \mathcal{Q}_{t, T}$. Hence, according to Neveu [100], for any $Z \in \mathcal{Q}_{t, T}$ there exists a sequence $\left(Y^{n}\right) \in \mathcal{A}_{t, T}$ such that $\gamma_{t, T}(Z)=\lim _{n \rightarrow \infty} \uparrow \mathrm{E}\left[-Z Y^{n} \mid \mathcal{F}_{t}\right]$. By use of monotone convergence we, then, obtain

$$
\mathrm{E}\left[\kappa \gamma_{t, T}(Z)\right]=\lim _{n \rightarrow \infty} \mathrm{E}\left[-\kappa Z Y^{n}\right] \leq \sup _{Y \in \mathcal{A}_{t, T}} \mathrm{E}[-\kappa Z Y]
$$

Conversely, it follows directly from (4.14) that $\mathrm{E}\left[\kappa \gamma_{t, T}(Z)\right] \geq \sup _{Y \in \mathcal{A}_{t, T}} \mathrm{E}[-\kappa Z Y]$ and, hence, we have equality. Consequently $Z \rightarrow \mathrm{E}\left[\kappa \gamma_{t, T}(Z)\right], Z \in \mathcal{Q}_{t, T}$, is convex and weakly lower semicontinuous as it is the point-wise supremum of a family of affine and weakly continuous functions. 
For a general convex risk measure, this set is not weakly compact. However, as we consider risk measures which are continuous from below, the associated level sets are. In particular, for a coherent risk-measure for which $\gamma \in\{0, \infty\}$, it follows that

$$
\mathcal{Q}_{t, T}^{a}:=\left\{\left.\mathbb{Q} \ll \mathbb{P}\right|_{\mathcal{F}_{T}}: \mathbb{Q}=\mathbb{P} \text { on } \mathcal{F}_{t} \text { and } \gamma_{t, T}(\mathbb{Q})=0, \text { a.s. }\right\}
$$

is weakly compact. If further $\mathcal{Q}_{t, T}^{a} \subseteq\left\{\left.\mathbb{Q} \sim \mathbb{P}\right|_{\mathcal{F}_{T}}\right\}$, then the set in (4.15) is also weakly compact. An example of such a risk measure is considered in [64] (cf. also Theorem 3.16 in [78]). Naturally, in general Assumption 4.5 allows for much more flexibility, see also Section 4.5.

Time-consistent risk measures

For convex risk measures, time-consistency is characterized by the property (4.8). Indeed, this property is equivalent (cf. e.g. Theorem 4.5 in [43]) to $\rho$, given in (4.13), satisfying

$$
\rho_{s, T}(X)=\rho_{s, t}\left(-\rho_{t, T}(X)\right)
$$

One would expect this property (combined with Assumption 4.5) to be sufficient for e.g. Lemma 4.11 to hold. Indeed, assume that $U(x, T) \in L^{\infty}$, for $x \in \mathbb{R}$. For a fixed strategy $\bar{\pi} \in \mathcal{A}_{b d}$, the relation in (4.10) then reduces to

$$
\phi_{s, T}\left(U\left(X_{T}^{\bar{\pi}}, T\right)\right)=\phi_{s, t}\left(\phi_{t, T}\left(U\left(X_{T}^{\bar{\pi}}, T\right)\right)\right.
$$

where $\phi(X)=-\rho(X)$ and which, thus, holds true due to (4.16). Time-consistency of the valuefunction has also been verified for the choice of specific models and utility functions (see, among others, [64]). We leave proving our results under this assumption for future research ${ }^{4}$ and restrict ourselves to the stronger Assumption 4.10. Note that any time-consistent coherent risk measure admits the pasting-property (4.9) (cf. Corollary 1.26 in [2]). In fact, in our case when all measures in $\mathcal{Q}_{t, T}$ are equivalent to the reference measure, even more explicit results hold for these risk measures (for results on the relation between stable sets and time-consistent coherent risk-measures, we refer to $[32,43,78])$.

\footnotetext{
${ }^{4}$ Proving that (4.8) implies (4.16) (see, e.g., Proposition 1.7 in [12]) exploits the fact that a conditional risk measure admitting the representation (4.13) also admits the equivalent representation

$$
\rho_{t, T}(X):=\mathbb{Q}-\operatorname{ess}_{R \in \mathcal{Q}_{t, T}(\mathbb{Q})}\left(\mathrm{E}^{R}\left[-X \mid \mathcal{F}_{t}\right]-\gamma_{t, T}(R)\right),
$$

where$$
\mathcal{Q}_{t, T}(\mathbb{Q}):=\left\{\left.R \ll \mathbb{P}\right|_{\mathcal{F}_{T}}:\left.R\right|_{\mathcal{F}_{t}}=\left.\mathbb{Q}\right|_{\mathcal{F}_{t}}, \mathrm{E}^{R}\left[\gamma_{t, T}(R)\right]<\infty\right\},
$$

for any $\mathbb{Q} \ll \mathbb{P}$. Similarly, in order to prove that (4.8) implies (4.10), one would need to verify that the set $\mathcal{Q}_{t, T}$ (as defined in (4.15)) in (4.2) can be replaced by the set of measure satisfying the additional assumption that they equal $\mathbb{Q}$ on $\mathcal{F}_{t}$, for some $\mathbb{Q} \sim \mathbb{P}$; this without affecting the value-function.
} 


\subsection{Non-volatile robust criteria in a Brownian filtration}

In this section we study a specific type of robust forward criteria, characterized by a specific evolutionary property, referred to as non-volatile criteria. This is done within a Brownian filtration and the dual domain. The discussion is formal. Our aim is to illustrate the flexibility of the notion of robust forward criteria and the fact that interesting preferences might be obtained from additional evolutionary requirements. In particular, non-volatile criteria are linked to a specific PDE (cf. equation (4.34) below) and the difficulties associated with this equation are discussed. An explicit example which results from this discussion is studied in detail in Section 4.5.

\subsubsection{The Brownian setup}

We specify further the general setup introduced in Section 4.2.1. We consider a filtration generated by a two-dimensional $\mathbb{P}$-Brownian motion $W_{t}=\left(W_{t}^{1}, W_{t}^{2}\right), t \geq 0$, and assume that the market consists of a risk-less and risky asset denoted, respectively, by $S_{t}^{0}$ and $S_{t}, t \geq 0$. Denoting the asset prices in units of $S^{0}$, we have $S_{t}^{0} \equiv 1$ while the dynamics of the risky asset is given by

$$
d S_{t}=S_{t}\left(\lambda_{t} d t+\sigma_{t} d W_{t}^{1}\right)
$$

for some $\mathbb{F}$-progressively measurable processes $\sigma_{t}, \sigma_{t} \neq 0$ a.s., and $\lambda_{t}, t \geq 0$, where the latter is referred to as the market price of risk.

At this point, we stress that the reference model $\mathbb{P}$ is not available to the agent. Rather, she will estimate a model, denoted by $\hat{\mathbb{P}}$, which she thinks most likely to be the accurate one. We assume that $\hat{\mathbb{P}} \sim \mathbb{P}$ and denote by $\left(\hat{\lambda}_{t}, \hat{\eta}_{t}\right), t \geq 0$, the process for which, for all $T>0$,

$$
\left.\frac{\mathrm{d} \hat{\mathbb{P}}}{\mathrm{dP}}\right|_{\mathcal{F}_{T}}=\mathcal{E}\left(\int_{0}^{\cdot}\left(\hat{\lambda}_{s}-\lambda_{s}\right) d W_{s}^{1}+\int_{0}^{\cdot} \hat{\eta}_{s} d W_{s}^{2}\right)_{T} .
$$

This readily implies that

$$
d S_{t}=S_{t}\left(\hat{\lambda}_{t} d t+\sigma_{t} d \hat{W}_{t}^{1}\right)
$$

for some $\hat{\mathbb{P}}$ Brownian motion $\hat{W}_{t}, t \geq 0$. Consequently, the agent will consider investment criteria formulated with respect to the model $\hat{\mathbb{P}}$. For a given criterion, this can be addressed by a change of the reference measure. However, in order to stress that the estimated measure $\hat{\mathbb{P}}$ might differ from (the unknown) $\mathbb{P}$ and illustrate the implications thereof, we keep this explicit notation throughout the section (see further discussion on page 110).

Let $\mathcal{P}$ denote the set of all $\mathbb{F}$-progressively measurable processes $\left(\nu_{t}\right)_{t \geq 0}$ such that $\int_{0}^{T} \nu_{t}^{2} d t<\infty$ a.s. for all $T>0$. We assume that $\left(\hat{\lambda}_{t}\right)$ is in $\mathcal{P}$. Next, let

$$
\mathcal{M}:=\left\{\nu \in \mathcal{P}: Z_{t}^{\nu} \text { is a } \hat{\mathbb{P}} \text { martingale on }[0, \infty)\right\},
$$


where

$$
Z_{t}^{\nu}:=\mathcal{E}\left(-\int \hat{\lambda}_{s} d \hat{W}_{s}^{1}-\int \nu_{s} d \hat{W}_{s}^{2}\right)
$$

We assume that $\nu_{t} \equiv 0$ is in $\mathcal{M}$ so that the assumption of NFLVR on finite horizons is satisfied with respect to $\hat{\mathbb{P}}$ (or, equivalently, $\mathbb{P}$ ). Also note that a density process $Z_{t}$ is in $\mathcal{Z}^{e}$ (defined with respect to $\hat{\mathbb{P}}$ ) if and only if is of the form $Z_{t}^{\nu}$, for some $\nu \in \mathcal{M}$. Any measure $\mathbb{Q}$ such that $\mathbb{Q} \sim \hat{\mathbb{P}}$ on $\mathcal{F}_{T}$, admits a process $\eta_{t}=\left(\eta_{t}^{1}, \eta_{t}^{2}\right) \in \mathcal{P} \times \mathcal{P}, t \leq T$, such that $\left.\frac{\mathrm{d} \mathbb{Q}}{\mathrm{d} \mathbb{P}}\right|_{\mathcal{F}_{T}}=D_{T}^{\eta}$, where

$$
D_{t}^{\eta}:=\mathcal{E}\left(\int_{0}^{\cdot} \eta_{s}^{1} d \hat{W}_{s}^{1}+\int_{0}^{\cdot} \eta_{s}^{2} d \hat{W}_{s}^{2}\right)_{t}
$$

is a martingale on $[0, T]$. We write $\mathbb{Q}=\mathbb{Q}^{\eta}$ and assign it a penalty given by ${ }^{5}$

$$
\gamma_{t, T}(\mathbb{Q}):=\mathrm{E}^{\mathbb{Q}}\left[\int_{t}^{T} g_{u}\left(\eta_{u}\right) d u \mid \mathcal{F}_{t}\right]
$$

for some function $g:[0, \infty) \times \mathbb{R}^{2} \rightarrow[0, \infty)$, such that $g_{t}(\cdot)$ is convex, lower-semicontinuous and satisfies the so called coercivity condition that $g_{t}(\eta) \geq-a+b|\eta|^{2}$ for some constants $a$ and $b$ (cf. (8.6) in [46]). For example, the choice of $g_{t}(\eta)=|\eta|^{2}+\infty \mathbf{1}_{|\eta|>\bar{g}}$ for some constant $\bar{g}>0$, ensures that $\gamma_{t, T}$ satisfies both Assumptions 4.5 and 4.10. ${ }^{6}$ We let $\mathcal{Q}=\cap_{T>0} \mathcal{Q}_{0, T}$.

Under the assumptions of Lemma 4.14, for a dual random field $V(y, t)$ and a family of penalty functions $\gamma_{t, T}$ of the type (4.23) to be self-generating in the dual domain, it is then sufficient that the process

$$
D_{t}^{\eta} V\left(y Z_{t}^{\nu} / D_{t}^{\eta}, t\right)+\int_{0}^{t} D_{s}^{\eta} g\left(\eta_{s}\right) d s
$$

is a $\mathbb{P}$-sub-martingale for all $\nu \in \mathcal{M}$ and $\eta \in \mathcal{Q}$ and a martingale for some $\eta^{*}$ and $\nu^{*}$. Equivalently, it is sufficient to require that for all $\nu \in \mathcal{M}$ and $\eta \in \mathcal{Q}$ the process

$$
M_{t}^{\eta \nu}:=V\left(y Z_{t}^{\nu} / D_{t}^{\eta}, t\right)+\int_{0}^{t} g\left(\eta_{s}\right) d s
$$

is a $\mathbb{Q}^{\eta}$-sub-martingale, with $\mathbb{Q}^{\eta}$ defined via $\left.\frac{\mathrm{d} \mathbb{Q}}{\mathrm{dP}}\right|_{\mathcal{F}_{t}}=D_{t}^{\eta}$, and, there exist $\nu^{*}$ and $\eta^{*}$ for which it is a martingale.

Given the assumption of a Brownian filtration, it is natural to assume that the dual value field admits the Itô-decomposition

$$
d V(y, t)=b(y, t) d t+a(y, t) d \hat{W}_{t}
$$

For future use, we establish:

\footnotetext{
${ }^{5}$ We recall that according to [33], it holds within a Brownian filtration that a dynamic penalty function is time-consistent (cf. (4.8)) if and only if it is representable as in (4.23) for some $g_{t}(\cdot)$.

${ }^{6}$ This follows e.g. from Lemma 3.1 in [64] and the fact that $\mathcal{Q}_{t, T}$ is weakly compact if and only if it is closed in $L^{0}$, see also discussion below Assumption 4.5 above.
} 
Lemma 4.16. The process $M_{t}^{\eta \nu}, t \geq 0$, given in (4.24) with $V(y, t)$ given in (4.25), follows the dynamics

$$
d M_{t}^{\eta \nu}=\tilde{b}\left(y \frac{Z_{t}^{\nu}}{D_{t}^{\eta}}, t\right) d t+\tilde{a}\left(y \frac{Z_{t}^{\nu}}{D_{t}^{\eta}}, t\right) \cdot d W_{t}^{\eta},
$$

where $W_{t}^{\eta}$ is a Brownian motion with respect to the measure $\mathbb{Q}$ defined via $\left.\frac{\mathrm{d} \mathbb{Q}}{\mathrm{d} \mathbb{P}}\right|_{\mathcal{F}_{t}}=D_{t}^{\eta}$, and where $\tilde{a}$ is some progressively measurable process and where $\tilde{b}(y, t)$ is given by

$$
\begin{aligned}
\tilde{b}(y, t)= & g\left(\eta_{t}\right)+\frac{y^{2} V_{y y}(y, t)}{2}\left(\left(\eta_{t}^{1}+\hat{\lambda}_{t}\right)^{2}+\left(\eta_{t}^{2}+\nu_{t}\right)^{2}\right) \\
& +b(y, t)+a(y, t) \cdot \eta_{t}-y a_{y}(y, t) \cdot\left(\eta_{t}^{1}+\hat{\lambda}_{t}, \eta_{t}^{2}+\nu_{t}\right)^{\prime} .
\end{aligned}
$$

Proof. First, we claim that

$$
d\left(\frac{Z_{t}^{\nu}}{D_{t}^{\eta}}\right)=-\frac{Z_{t}^{\nu}}{D_{t}^{\eta}}\left[\left(\eta_{t}^{1}+\hat{\lambda}_{t}\right) d W_{t}^{\eta, 1}+\left(\eta_{t}^{2}+\nu_{t}\right) d W_{t}^{\eta, 2}\right],
$$

where $\left(W_{t}^{\eta, 1}, W_{t}^{\eta, 2}\right)$ is a Brownian motion under the measure $\mathbb{Q}$ defined via $\left.\frac{\mathrm{d} \mathbb{Q}}{\mathrm{dP}}\right|_{\mathcal{F}_{t}}=D_{t}^{\eta}$. Indeed, according to (4.21) and (4.22), it holds that

$$
\frac{Z_{t}^{\nu}}{D_{t}^{\eta}}=\exp \left\{-\int_{0}^{t} \eta_{s}^{1}+\hat{\lambda}_{s} d W_{s}^{1}-\int_{0}^{t} \eta_{s}^{2}+\nu_{s} d W_{s}^{2}+\frac{1}{2} \int_{0}^{t}\left(\eta_{s}^{1}\right)^{2}-\hat{\lambda}_{s}^{2}+\left(\eta_{s}^{2}\right)^{2}-\nu_{s}^{2} d s\right\} .
$$

Use of Girsanov yields

$$
\begin{aligned}
-\int_{0}^{t} \eta_{s}^{1}+\hat{\lambda}_{s} d W_{s}^{1}+\frac{1}{2} \int_{0}^{t}\left(\eta_{s}^{1}\right)^{2}-\hat{\lambda}_{s}^{2} d s & =-\int_{0}^{t}\left(\eta_{s}^{1}+\hat{\lambda}_{s}\right)\left(d W_{s}^{\eta, 1}+\eta_{s}^{1} d s\right)+\frac{1}{2} \int_{0}^{t}\left(\eta_{s}^{1}\right)^{2}-\hat{\lambda}_{s}^{2} d s \\
& =-\int_{0}^{t} \eta_{s}^{1}+\hat{\lambda}_{s} d W_{s}^{\eta, 1}-\frac{1}{2} \int_{0}^{t}\left(\hat{\lambda}_{s}+\eta_{s}^{1}\right)^{2} d s
\end{aligned}
$$

and a similar result for the second part of $(4.27)$, with $\left(\tilde{W}_{s}^{1}, \tilde{W}_{s}^{2}\right)$ as given above. Hence, we obtain

$$
\frac{Z_{T}^{\nu}}{D_{t}^{\eta}}=\exp \left\{-\int_{0}^{t} \eta_{s}^{1}+\hat{\lambda}_{s} d W_{s}^{\eta, 1}-\int_{0}^{t} \eta_{s}^{2}+\nu_{s} d W_{s}^{\eta, 2}-\frac{1}{2} \int_{0}^{t}\left(\hat{\lambda}_{s}+\eta_{s}^{1}\right)^{2}+\left(\nu_{s}+\eta_{s}^{2}\right)^{2} d s\right\}
$$

and the claim follows. Then, in turn, use of Itô-Ventzell's formula yields (the argument $\left(y Z_{t} / D_{t}^{\eta}, t\right)$ suppressed),

$$
\begin{aligned}
d M_{t}= & g\left(\eta_{t}\right) d t+\frac{1}{2} V_{y y}\left(y \frac{Z_{t}^{\nu}}{D_{t}^{\eta}}\right)^{2}\left(\left(\eta_{t}^{1}+\hat{\lambda}_{t}\right)^{2}+\left(\eta_{t}^{2}+\nu_{t}\right)^{2}\right) d t \\
& -V_{y} y \frac{Z_{t}^{\nu}}{D_{t}^{\eta}}\left(\left(\eta_{t}^{1}+\hat{\lambda}_{t}\right) d W_{t}^{\eta, 1}+\left(\eta_{t}^{2}+\nu_{t}\right) d W_{t}^{\eta, 2}\right) \\
& +b d t+a^{1}\left[d W_{t}^{\eta, 1}+\eta_{t}^{1} d t\right]+a^{2}\left[d W_{t}^{\eta, 2}+\eta_{t}^{2} d t\right]-y \frac{Z_{t}^{\nu}}{D_{t}^{\eta}}\left[a_{y}^{1}\left(\eta_{t}^{1}+\hat{\lambda}_{t}\right)+a_{y}^{2}\left(\eta_{t}^{2}+\nu_{t}\right)\right] d t \\
= & \left\{g\left(\eta_{t}\right)+\frac{1}{2} V_{y y}\left(y \frac{Z_{t}^{\nu}}{D_{t}^{\eta}}\right)^{2}\left(\left(\eta_{t}^{1}+\hat{\lambda}_{t}\right)^{2}+\left(\eta_{t}^{2}+\nu_{t}\right)^{2}\right)\right. \\
& \left.+b+a^{1} \eta_{t}^{1}+a^{2} \eta_{t}^{2}-y \frac{Z_{t}^{\nu}}{D_{t}^{\eta}}\left(a_{y}^{1}\left(\eta_{t}^{1}+\hat{\lambda}_{t}\right)+a_{y}^{2}\left(\eta_{t}^{2}+\nu_{t}\right)\right)\right\} d t \\
& -V_{y} y \frac{Z_{t}^{\nu}}{D_{t}^{\eta}}\left(\left(\eta_{t}^{1}+\hat{\lambda}_{t}\right) d W_{t}^{\eta, 1}+\left(\eta_{t}^{2}+\nu_{t}\right) d W_{t}^{\eta, 2}\right)+a \cdot d W_{t}^{\eta} .
\end{aligned}
$$

The result follows by rearrangement of terms. 


\subsubsection{Pinning down of robust forward criteria from the initial condi- tion}

In the model-specific (non-robust) case, the robust forward performance are not uniquely specified from the initial condition. This due to the flexibility in the volatility structure ${ }^{7}$. We expect a similar observation to hold in the robust case but subject to specifying the penalty function. We present here a formal discussion of the logarithmic example which motivates our conjecture.

Let $V(y, t)$ be of logarithmic type in that it admits the representation

$$
V(y, t)=-\ln y+\int_{0}^{t} b_{s} d s+\int_{0}^{t} a_{s} \cdot d \hat{W}_{s}
$$

for some processes $b_{t}$ and $a_{t}$ which are independent of $y$. For this choice, $V_{y y}(y, t)=1 / y^{2}$. Hence, formally, it follows from Lemma 4.16 that

$$
d M_{t}^{\eta \nu}=\left\{g\left(\eta_{t}\right)+\frac{1}{2}\left(\left(\eta_{t}^{1}+\hat{\lambda}_{t}\right)^{2}+\left(\eta_{t}^{2}+\nu_{t}\right)^{2}\right)+b_{t}+a_{t} \cdot \eta_{t}\right\} d t+\tilde{a}\left(y \frac{Z_{t}^{\nu}}{D_{t}^{\eta}}, t\right) \cdot d W_{t}^{\eta} .
$$

At each point in time, say $t$, the drift-term attains its infimum with respect to $\nu$ for the choice of $\nu_{t}=-\eta_{t}^{2}$, for which the $\nu$-dependent term of the drift-term disappears. According to Lemma 4.14, in order for $V(y, t)$ to be self-generating, $M_{t}^{\eta \nu}$ must be a sub-martingale for each choice of $\nu$ and $\eta$ and a martingale at optimum. Hence, following (4.30), it must hold that

$$
b_{t}=-\inf _{\eta}\left\{g(\eta)+\frac{\left(\eta^{1}+\hat{\lambda}_{t}\right)^{2}}{2}+a_{t} \cdot \eta\right\}, \quad \text { a.s. }, t \geq 0 .
$$

We see, on a formal level, that for a given initial condition and a fixed penalty function $g(\cdot)$, there might still exist multiple robust forward criteria. These may be catalogue by their volatility: the drift is then specified via (4.31). In the next two sections we will be interested respectively in the case when volatility is a function of stochastic factors and when it is zero, i.e. in non-volatile criteria.

\footnotetext{
${ }^{7}$ Indeed, for the non-robust case (within a Brownian filtration, cf. [89] and [97] for details), it holds that a random field is a forward criterion if it, for all times $t \geq 0$, satisfies the SPDE

$$
d U(x, t)=\frac{1}{2} \frac{\left|\lambda_{t} U_{x}(x, t)+\sigma_{t} \sigma_{t}^{+} a_{x}(x, t)\right|^{2}}{U_{x x}(x, t)} d t+a(x, t) d W_{t},
$$

equipped with the initial condition $U(x, 0)=u_{0}(x)$. Similarly, it can be shown that the value-function corresponding to the standard utility maximization problem (under some regularity conditions) must satisfy the Backward SPDE (4.28) equipped with the terminal condition $U(x, T)=U(x)$.

A solution to the latter is then a pair of parameter-dependent processes $U(x, t)$ and $a(x, t)$, which satisfy the equation as well as the terminal condition, and which are simultaneously obtained when solving this Backward SPDE. In particular, under some regularity conditions, the solution pair associated with a given terminal condition is unique. The presence of the volatility $a(x, t)$ implies, however, that there might exist multiple stochastic terminal conditions, for all of which the associated solution satisfies $U_{0}(x)=u_{0}(x)$. Hence, if one allows for stochastic terminal utility functions, there might exist multiple solutions to the inverse investment problem. In particular, there is a one-to-one correspondence between such solutions to the inverse investment problem and admissible volatility processes $a(x, t)$.
} 


\subsubsection{Fields with volatility in function of stochastic factors}

One specific type of criteria are obtained by requiring, within a stochastic factor model, the forward criterion to be a deterministic function of the involved factors. Note that this is an additional assumption as forward criteria, unlike value-functions defined with respect to deterministic utility functions, need not admit such a representation despite the underlying market model being driven by stochastic factors.

For the logarithmic case in Section 4.4.2, given that $\mu_{t}$ and $\sigma_{t}$ are deterministic functions of some stochastic factor $Z_{t}$, that amounts to assume that $V(y, t)=-\ln y+v\left(Z_{t}, t\right)$ for some deterministic function $v(z, t)$. Condition (4.31) then reduces ${ }^{8}$ to a $\mathrm{PDE}$ for that deterministic function $v(z, t)$. Naturally, that PDE will be similar to the one obtained for the value-function associated with the ambiguity averse investment problem with a fixed finite horizon and logarithmic terminal utility set within the same market model (cf. Theorem 2.1 in [65]). However, for the forward case the corresponding equation is equipped with an initial condition and to be solved forward in time for all $t \geq 0$. For a general discussion of the issues related to the fact that forward criteria give rise to HJB-equations equipped with initial conditions and to be solved on the entire positive line, we refer to [99] where similar standard (non-robust) forward criteria were studied (see also Section 3.1.4). Characterisation of initial conditions for which a solution exists is highly nontrivial. On the other hand, given existence for a specific initial condition and penalty function, the solution $v(z, t)$ will be unique under mild conditions. That is, there is a unique way of time-homogeneously propagating the preferences forward under such assumptions.

\subsubsection{Non-volatile criteria}

Another interesting class of preferences are obtained by requiring the volatility of the dual field in $(4.25)$ to be zero: $a(y, t) \equiv 0$. We refer to these criteria as non-volatile. For standard forward criteria, this additional assumption has proven to provide an interesting class of preferences (cf. $[8,96]$ and Section 3.1.4).

In order to ease notation, the process $b(y, t)$ is henceforth denoted by $V_{t}(y, t)$. Consequently,

$$
d V(y, t)=V_{t}(y, t) d t .
$$

\footnotetext{
${ }^{8}$ Given that the stochastic factor has dynamics given by $d Z_{t}=j\left(Z_{t}\right) d t+d W_{t}^{2}$ it follows from the specific form of $V(y, t)$ that

$$
d V(y, t)=\left\{v_{t}\left(Z_{t}, t\right)+v_{z}\left(Z_{t}, t\right) j\left(Z_{t}\right)+\frac{1}{2} v_{z z}\left(Z_{t}, t\right)\right\} d t+v_{z}\left(Z_{t}, t\right) d W_{t}
$$

which substituted into equation (4.31) yields

$$
v_{t}(z, t)+v_{z}(z, t) j(z)+\frac{1}{2} v_{z z}(z, t)+\inf _{\eta}\left\{g(\eta)+\frac{\left(\eta^{1}+\lambda(z)\right)^{2}}{2}+v_{z}(z, t) \eta^{2}\right\}=0, \quad t \geq 0 .
$$


According to Lemma 4.16, the evolution of $M_{t}^{\eta \nu}, t \geq 0$, is then described by

$d M_{t}^{\eta \nu}=\left[V_{t}\left(y \frac{Z_{t}^{\nu}}{D_{t}^{\eta}}, t\right)+g\left(\eta_{t}\right)+\left(y \frac{Z_{t}^{\nu}}{D_{t}^{\eta}}\right)^{2} V_{y y}\left(y \frac{Z_{t}^{\nu}}{D_{t}^{\eta}}, t\right) \frac{\left(\eta_{t}^{1}+\hat{\lambda}_{t}\right)^{2}+\left(\eta_{t}^{2}+\nu_{t}\right)^{2}}{2}\right] d t+\tilde{a}_{t} \cdot d W_{t}^{\eta}$,

for some process $\tilde{a}_{t}, t \geq 0$. As $V(y, t)$ is convex in its spatial variable, it follows at each point in time $t$, the drift-term attains its infimum with respect to $\nu$ for the choice of $\nu_{t}=-\eta_{t}^{2}$. In particular, the last term of the drift-term disappears for this choice and, thus, it follows from Theorem 4.14 (cf. equation (4.24)) that in order to be a forward criterion, the random convex function $V(y, t)$ must solve the equation

$$
V_{t}(y, t)+\inf _{\eta}\left\{g(\eta)+\frac{y^{2} V_{y y}(y, t)}{2}\left(\eta_{t}^{1}+\hat{\lambda}_{t}\right)^{2}\right\}=0, \quad \text { a.s. }, t \geq 0 .
$$

Although only formally derived, the above equation might thus be viewed as a type of dual Hamilton-Jacobi-Bellman equation. In particular, given existence of a solution to equation (4.34), a verification theorem establishing the sufficiency of this condition might also be proven. It would verify that every convex solution to equation (4.34) constitutes a forward criterion. However, equation (4.34) is rather hard to handle. The aim in the remainder of this section is to illustrate this and to point out some specific features of this equation.

Equation (4.34) is a random equation which is satisfied pathwise by the process $V(y, t)$, which is of finite variation. It is a random PDE as opposed to a SPDE due to the restriction to nonvolatile criteria. In particular, this implies that for a given initial condition and fixed penalty function for which a solution exists, that solution is unique (under some regularity conditions). Equivalently, for a given penalty function, there is a unique way (if it exists) to propagate the preferences - forwards in time. This is inline with our discussion in Section 4.4.2 above.

For a fixed path, equation (4.34) coincides with the one appearing for the case of deterministic utility fields within a log-normal market (cf. the stochastic factor example above with $\mu$ and $\sigma$ constant). In particular, on any fixed interval $[0, T)$, it then coincides with the Hamilton-JacobiBellman equation for a (deterministic) value-function within a log-normal model. The underlying model (cf. (4.18)) is, however, not log-normal and is possibly incomplete. Rather than being an effect of the market model, it is the non-volatility assumption that forces the criterion to be of a very specific stochastic form which, in turn, renders the behaviour path-wise myopic; that is to say, equivalent to that of a log-normal market.

For the particular case of no model-uncertainty, that is to say when $g(\eta)=\infty, \eta \neq 0$, equation (4.34) reduces to

$$
V_{t}(y, t)+\frac{\hat{\lambda}_{t}^{2}}{2} y^{2} V_{y y}(y, t)=0 \quad \text { a.s. }, \quad t \geq 0 .
$$


We recall that a random convex function $V(y, t)$ satisfies equation (4.35) if and only if its associated primal field satisfies equation (3.16); cf. Lemma 3.6. Hence, equation (4.35) characterizes (standard) non-volatile forward criteria in the market with market price of risk $\left(\hat{\lambda}_{t}\right)$. As discussed above, this equation is closely related to the (ill-posed) backward heat equation and the set of admissible initial conditions and the associated solutions have been obtained by use of Widder's theorem (cf. Section 3.1.4.2 for details and further references). As noted in Section 4.4.2, similar ill-posed related aspects are present also for the more general equation (4.34). In addition to that, equation (4.34) is also highly non-linear. In order to illustrate this, consider fixing a path and then searching for an optimal $\eta$ in feed-back form. Since $V_{y y} \geq 0$ and $g$ is convex, the expression appearing in (4.34) is a convex function of $\eta$. Hence, if there exists an $\eta^{*}$ for which the first order condition

$$
\left(\eta+\lambda_{t}\right) y^{2} V_{y y}(y, t)+g^{\prime}(\eta)=0
$$

is satisfied, then that $\eta^{*}$ must correspond to a minimum. In particular, if $g(\eta)$ has a minimum for $\eta=0$, then it holds that $\eta^{*} \in\left[-\hat{\lambda}_{t}, 0\right]$ (indeed, for $\eta<-\hat{\lambda}_{t}$ it holds that $\eta+\hat{\lambda}_{t}<0$ and $g^{\prime}(\eta)<0$, which contradicts (4.36). Similarly, for $\eta>0$, it holds that $\eta+\hat{\lambda}_{t}>0$ and $g^{\prime}(\eta)>0$ ). Formally, one could thus solve (4.36) for $\eta^{*}$ and then substitute for $\eta^{*}$ in (4.34) and then, in turn, solve for $V(y, t)$. The resulting equation for $V(y, t)$ will, however, be highly non-linear as $\eta^{*}$ depends on $V_{y y}$. In summary, the difficulties arising from ill-posed related issues as well as non-linearity, renders it hard to analyze equation (4.34). In the next section, we consider a specific, particularly interesting, example which we can solve explicitly.

\subsection{Non-volatile logarithmic criteria - an illustrative ex- ample}

In this section, we consider non-volatile criteria of logarithmic type. The example is of particular interest as it gives theoretical justification to fractional Kelly strategies often used in practice by large investment funds. More precisely, the agent estimates (dynamically) the market growth (Kelly) strategy $\hat{X}$ and invests a (dynamically adjusted) fraction of her wealth in $\hat{X}$. The leverage, in our framework, has the interpretation of agent's confidence in his estimate of $\hat{X}$. Despite its very specific form, the explicit solution also illustrates two important and general facts about robust forward criteria. Namely, that for each robust forward criterion, there exists a (standard) forward criterion in a specific auxiliary market as well as a specific (standard) forward criterion in the reference market, giving rise to the same optimal behaviour. These properties are further discussed also for more general criteria. 


\subsubsection{Logarithmic example - main result}

We consider the same Brownian framework as set out in the previous section (cf. (4.18)) and assume that the investor considers forward criteria defined with respect to the estimated model $\hat{\mathbb{P}}$. However, here we let $\left(\pi_{t}\right)_{t \geq 0}$, denote the fraction of wealth invested in the risky asset. The associated wealth process follows the dynamics

$$
d X_{t}^{\pi}=\pi_{t} X_{t}^{\pi} \sigma_{t}\left(\hat{\lambda}_{t} d t+d \hat{W}_{t}^{1}\right), \quad X_{0}=x
$$

The set of admissible strategies is here defined as follows:

$$
\mathcal{A}:=\left\{\pi:\left(\pi_{t}\right) \text { adapted, }\left(X_{t}^{\pi}\right) \text { well-defined and } X_{t}^{\pi}>0 \text { a.s. for all } t>0\right\} .
$$

and we also write $\mathcal{A}^{x}$ when we want to stress the initial wealth $X_{0}=x$. Finally, we denote by $\mathcal{A}_{t}^{x}$ the strategies on in $\mathcal{A}$ on $[t, \infty)$ and taking $X_{t}^{\pi}=x$.

Given the Brownian filtration, a time-consistent penalty function admits representation (4.23) for some admissible function $g_{t}(\cdot)$. We also consider a slightly more general penalty function

$$
\gamma_{t, T}\left(\mathbb{Q}^{\eta}\right):= \begin{cases}\mathrm{E}^{\mathbb{Q}^{\eta}}\left[\int_{t}^{T} \frac{\delta_{u}}{2}\left|\eta_{u}\right|^{2} d u \mid \mathcal{F}_{t}\right] & \text { if } \mathrm{E}^{\mathbb{Q}^{\eta}}\left[\int_{t}^{T} \hat{\lambda}_{s}^{2} d s\right]<\infty \\ +\infty & \text { otherwise }\end{cases}
$$

for some adapted, non-negative process $\left(\delta_{t}\right)$ which controls the strength of the penalisation. The investor is aware that $\hat{\mathbb{P}}$ may be an inaccurate estimate of the market (cf. p. 103) and $\left(\delta_{t}\right)$ quantifies her trust in $\hat{\mathbb{P}}$. Note that $\gamma_{t, T}$ may fail to satisfy Assumptions 4.5 and 4.10. In particular, $\mathcal{Q}_{t, T}$ in (4.1) may not be weakly compact. This is not a problem since, for this example, we present a direct proof. Finally, we assume that there exists $\kappa>1 / 2$ such that $\hat{\mathrm{E}}\left[\exp \left(\kappa \int_{0}^{T} \hat{\lambda}_{s}^{2} d s\right)\right]<\infty$ for all $T>0$. This is a convenient integrability assumption which can be interpreted as $\hat{\mathbb{P}}$ being reasonable. Note that it implies in particular, by Novikov's condition, that $\left(Z_{t}^{\nu}\right)$ in $(4.21)$ with $\nu \equiv 0$ is a $\hat{\mathbb{P}}$-martingale.

Proposition 4.17. Given the investor's choice of $\left(\hat{\lambda}_{t}\right)$ and $\left(\delta_{t}\right)$ as above, let

$$
\bar{\eta}_{t}:=\left[-\hat{\lambda}_{t} /\left(1+\delta_{t}\right), 0\right] \quad \text { and } \quad \bar{\pi}_{t}:=\frac{\delta_{t}}{1+\delta_{t}} \frac{\hat{\lambda}_{t}}{\sigma_{t}}
$$

and

$$
U(x, t):=\ln x-\frac{1}{2} \int_{0}^{t} \frac{\delta_{s}}{1+\delta_{s}} \hat{\lambda}_{s}^{2} d s, \quad t \geq 0, x \in \mathbb{R}_{+} .
$$

Recall that the penalty $\gamma$ is given by (4.37) and assume that $\bar{\eta} \in \mathcal{Q}_{0, T}$ for $T>0$. Then, for all $0 \leq t \leq T<\infty$

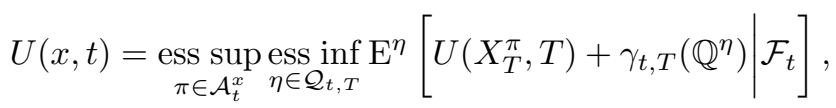

and the optimum is attained for the saddle point $(\bar{\eta}, \bar{\pi})$ as given in (4.38). 
The above result implies that the utility random field $U(x, t)$, given in (4.39), and the penalty function $\gamma_{t, T}$ in (4.37) constitute a robust forward criterion. For comparison, recall (cf. [96]) that the random field

$$
U(x, t)=\ln x-\frac{1}{2} \int_{0}^{t} \lambda_{s}^{2} d s, \quad t \geq 0, x \in \mathbb{R}_{+},
$$

constitutes a standard (non-volatile) forward criterion in the reference market $\mathbb{P}$ with market price of risk $\lambda_{t}, t \geq 0$. As the criterion (4.39) is logarithmic as well as non-volatile, the appropriate form of the drift-term could, formally, be obtained by substituting the dual Ansatz $V(y, t)=$ $-\ln y+\int_{0}^{t} b_{s} d s$ into either of equations (4.31) or (4.34). However, the proof below is carried out directly in the primal domain. For a comparison with the variational criterion featuring (deterministic) logarithmic utility at some fixed horizon $T>0$, we refer to [65] for a stochastic factor model and Theorem 4.5 in [82] for the non-Markovian case.

Proof. Fix $0 \leq t \leq T<\infty$. To alleviate the notation, let $L_{t}=\int_{0}^{t} \hat{\lambda}_{u} d \hat{W}_{u}$. We have, with $1 / p+1 / q=1$ and $1 / \tilde{p}+1 / \tilde{q}=1$,

$$
\begin{aligned}
\mathrm{E}^{\mathrm{Q}^{\bar{\eta}}}\left[\int_{0}^{T} \hat{\lambda}_{s}^{2} d s\right] & =\hat{\mathrm{E}}\left[D_{T}^{\bar{\eta}} \int_{0}^{T} \hat{\lambda}_{s}^{2} d s\right]=\hat{\mathrm{E}}\left[D_{T}^{\bar{\eta}}\langle L\rangle_{T} d s\right] \leq\left(\hat{\mathrm{E}}\left[\left(D_{T}^{\bar{\eta}}\right)^{p}\right]\right)^{1 / p}\left(\hat{\mathrm{E}}\left[\langle L\rangle_{T}^{q}\right]\right)^{1 / q} \\
& \leq\left(\hat{\mathrm{E}}\left[\mathrm{e}^{p \tilde{p} L_{T}-\frac{p^{2} \tilde{\tilde{p}}^{2}}{2}\langle L\rangle_{T}}\right]\right)^{\frac{1}{p \bar{p}}}\left(\hat{\mathrm{E}}\left[\mathrm{e}^{\kappa\langle L\rangle_{T}}\right]\right)^{\frac{1}{p \tilde{q}}}\left(\hat{\mathrm{E}}\left[\langle L\rangle_{T}^{q}\right]\right)^{1 / q}<\infty,
\end{aligned}
$$

where we took $\tilde{p}<2 \kappa$ and $p>1$ such that $\tilde{q}\left(\frac{p^{2} \tilde{p}}{2}-\frac{p}{2}\right)=\frac{p \tilde{p}(p \tilde{p}-1)}{2(\tilde{p}-1)}=\kappa$. It follows that $\gamma_{t, T}\left(\mathbb{Q}^{\bar{\eta}}\right)<\infty$. Let

$$
N_{u}^{\pi, \eta}:=U\left(X_{u}^{\pi}, u\right)+\int_{t}^{u} \frac{\delta_{s}}{2}\left|\eta_{s}\right|^{2} d s, \quad u \geq t
$$

Then, it suffices to show that $\mathrm{E}^{\bar{\eta}}\left[N_{T}^{\pi, \bar{\eta}} \mid \mathcal{F}_{t}\right] \leq N_{t}^{\pi, \bar{\eta}}$, for all $\pi \in \mathcal{A}_{t}^{x}$, and that $\mathrm{E}^{\eta}\left[N_{T}^{\bar{\pi}, \eta} \mid \mathcal{F}_{t}\right] \geq N_{t}^{\bar{\pi}, \eta}$, for all $\eta \in \mathcal{Q}_{t, T}$. For simplicity, and w.l.o.g., we show the claim in the case $t=0$. For $\pi \in \mathcal{A}^{x}$, the wealth process satisfies

$$
d X_{t}^{\pi}=\pi_{t} X_{t}^{\pi} \sigma_{t}\left[\left(\hat{\lambda}_{t}+\eta_{t}^{1}\right) d t+d W_{t}^{\eta}\right], \quad t \leq T, \quad X_{0}^{\pi}=x,
$$

where $W_{t}^{\eta}$ is a Brownian motion under $\mathbb{Q}^{\eta}$. Due to the form of $U(x, t)$ and $\bar{\pi}$, a straight-forward application of Itô's Lemma yields

$$
\begin{aligned}
d N_{t}^{\bar{\pi}, \eta}= & \frac{\delta_{t}}{1+\delta_{t}} \hat{\lambda}_{t}\left[\left(\hat{\lambda}_{t}+\eta_{t}^{1}\right) d t+d W_{t}^{\eta}\right]-\frac{1}{2}\left(\frac{\delta_{t}}{1+\delta_{t}} \hat{\lambda}_{t}\right)^{2} d t \\
& -\frac{1}{2} \frac{\delta_{t}}{1+\delta_{t}} \hat{\lambda}_{t}^{2} d t+\frac{\delta_{t}}{2}\left[\left(\eta_{t}^{1}\right)^{2}+\left(\eta_{t}^{2}\right)^{2}\right] d t \\
= & \frac{\delta_{t}}{1+\delta_{t}} \hat{\lambda}_{t} \eta_{t}^{1} d t+\frac{1}{2} \frac{\delta_{t}}{\left(1+\delta_{t}\right)^{2}} \hat{\lambda}_{t}^{2} d t+\frac{\delta_{t}}{1+\delta_{t}} \hat{\lambda}_{t} d W_{t}^{\eta}+\frac{\delta_{t}}{2}\left[\left(\eta_{t}^{1}\right)^{2}+\left(\eta_{t}^{2}\right)^{2}\right] d t \\
= & \frac{\delta_{t}}{2}\left[\left(\frac{\hat{\lambda}_{t}+\left(1+\delta_{t}\right) \eta_{t}^{1}}{1+\delta_{t}}\right)^{2}+\left(\eta_{t}^{2}\right)^{2}\right] d t+\frac{\delta_{t}}{1+\delta_{t}} \hat{\lambda}_{t} d W_{t}^{\eta}
\end{aligned}
$$


Note that $\delta_{t} /\left(1+\delta_{t}\right) \in(0,1)$ so by definition of $\gamma_{t, T}$ in $(4.37), \int_{0}^{t} \frac{\delta_{s}}{1+\delta_{s}} \hat{\lambda}_{s} d W_{s}^{\eta}$ is a martingale under $\mathbb{Q}^{\eta}$. It follows that $N_{t}^{\bar{\pi}, \eta}$ is a submartingale for all $\eta \in \mathcal{Q}_{0, T}$ and a martingale for $\bar{\eta}$ as specified in (4.38). On the other hand, it holds that

$$
\begin{aligned}
U\left(X_{T}^{\pi}, T\right)+\int_{0}^{T} g_{s}\left(\bar{\eta}_{s}\right) d s & =\ln X_{T}^{\pi}-\int_{0}^{T} \frac{1}{2} \frac{\delta_{s}}{1+\delta_{s}} \hat{\lambda}_{s}^{2}-\frac{1}{2} \frac{\delta_{s}}{\left(1+\delta_{s}\right)^{2}} \hat{\lambda}_{s}^{2} d s \\
& =\ln X_{T}^{\pi}-\frac{1}{2} \int_{0}^{T}\left[\frac{\delta_{s}}{1+\delta_{s}} \hat{\lambda}_{s}\right]^{2} d s=\ln X_{T}^{\pi}-\frac{1}{2} \int_{0}^{T}\left(\hat{\lambda}_{s}+\bar{\eta}_{s}^{1}\right)^{2} d s .
\end{aligned}
$$

Standard logarithmic utility maximization shows that under $\mathbb{Q}^{\bar{\eta}}, \mathrm{E}^{\bar{\eta}}\left[\ln X_{T}^{\pi}\right] \leq \mathrm{E}^{\bar{\eta}}\left[\ln X_{T}^{\bar{\pi}}\right]$ for any strategy $\pi \in \mathcal{A}^{x}$. We conclude that

$$
\mathrm{E}^{\bar{\eta}}\left[N_{T}^{\pi, \bar{\eta}}\right] \leq \mathrm{E}^{\bar{\eta}}\left[\ln X_{T}^{\bar{\pi}}\right]-\mathrm{E}^{\bar{\eta}}\left[\frac{1}{2} \int_{0}^{T}\left(\hat{\lambda}_{s}+\bar{\eta}_{s}^{1}\right)^{2} d s\right]=\ln x=N_{0},
$$

where the equality follows by a direct computation (see also e.g. p. 721 in [73]).

Remark 4.18. For $\delta_{t} \equiv \delta$, the penalty function defined in (4.37) corresponds to the entropic penalty function $\gamma(\mathbb{Q})=\delta H(\mathbb{Q} \mid \hat{\mathbb{P}})$. For each fixed horizon $T$ the investment problem can then be rewritten in the following way (cf. Remark 4.1 in [46]),

$$
\begin{aligned}
u(x, 0) & =\sup _{\pi} \inf _{\mathbb{Q}}\left(\mathrm{E}^{\mathbb{Q}}\left[U\left(X_{T}^{\pi}, T\right)\right]+\delta H(\mathbb{Q} \mid \hat{\mathbb{P}})\right) \\
& =\sup _{\pi}-\delta \ln \mathrm{E}^{\hat{\mathbb{P}}}\left[e^{-\frac{1}{\delta} U\left(X_{T}^{\pi}, T\right)}\right] .
\end{aligned}
$$

Consequently, the problem is equivalent to a standard utility maximization problem with respect to the modified utility function $\tilde{U}(x, T)=-e^{-\frac{1}{\delta} U(x, T)}$ in the market $\hat{\mathbb{P}}$. In this setting it is more natural to consider utility from inter-temporal consumption, cf. [18, 26, 40, 69, 86, 115]. Note, however, that since $\delta_{t}, t \geq 0$, is non-constant within our setting, this is not the case here.

\subsubsection{Fractional Kelly strategies - optimality and interpretation}

The investor's optimal behaviour described in Proposition 4.17 is remarkably simple and corresponds to strategies used in practice by some of the large fund mangers. The strategy, characterised by the optimal fraction of wealth to be invested in the risky asset in (4.38), is a fractional Kelly strategy. The investor invests in the growth optimal (Kelly) portfolio corresponding to her best estimate of the market price of risk $\hat{\lambda}$. However she is not fully invested but instead has a leverage proportional to her trust in the estimate $\hat{\lambda}$. If $\delta_{t} \nearrow \infty$ (infinite trust in the estimation), then $\bar{\pi}_{t} \nearrow \hat{\lambda}_{t} / \sigma_{t}$ which is the Kelly strategy associated with the most likely model $\hat{\mathbb{P}}$. On the other hand, if $\delta_{t} \searrow 0$ (no trust in the estimation), then $\bar{\pi}_{t} \searrow 0$ and the optimal behaviour is to invest nothing. 
We stress that $\hat{\lambda}$ and $\delta$ are investor's arbitrary inputs. They might be data driven and come from an elaborate dynamic estimation procedure, be expert driven or simply come from a black box. In particular there is no assumption that $\hat{\lambda}$ is a good estimate of the true market price of risk $\lambda$. In fact the latter never appears in the problem. It is crucial that the investor's utility function (4.39) evolves in function of the investor's perception of market leading to a time-consistent behaviour solving (4.17). This seem to capture well the investment practice - in reality an investor never knows the "true" model. Instead, she is likely to build (and keep updating) her best estimate thereof and act on it. This, as shown in Proposition 4.17, can lead to time-consistent optimal investment strategy.

In practice the leverage has often a risk interpretation, e.g. it is adjusted to achieve a targeted level of volatility for the fund. In our framework, it is interpreted in terms of confidence $\delta$ in the estimate $\hat{\lambda}$. In practice, the leverage is adjusted rarely in comparison to the dynamic updating of the estimate $\hat{\lambda}$. Similarly, in our framework, the trust in one's estimation methods is likely to be adjusted on a much slower scale than the changes to the estimate itself.

Finally, we note that the optimal strategy $\bar{\pi}$ in (4.38) can be also interpreted as the Kelly-strategy associated with an auxiliary market with market price of risk $\bar{\lambda}_{t}$ given by

$$
\bar{\lambda}_{t}:=\hat{\lambda}_{t}+\bar{\eta}_{t}^{1}=\frac{\delta_{t}}{1+\delta_{t}} \hat{\lambda}_{t}
$$

That is, the market price of risk that the agent thinks most likely - adjusted by the agent's trust in that estimation. This is closely related to the fact that the existence of the saddle-point $(\bar{\pi}, \bar{\eta})$ implies that the optimal investment associated with the robust criterion (4.37) and (4.39) coincides with the optimal investment corresponding to a non-volatile standard forward criterion (cf. (4.40)),

$$
U(x, t)=\ln x-\frac{1}{2} \int_{0}^{t} \bar{\lambda}_{s}^{2} d s, \quad t \geq 0, x \in \mathbb{R}_{+},
$$

specified in the market with the market-price of risk $\bar{\lambda}$. This is clear from the proof of Proposition 4.17 above.

\subsubsection{Existence of equivalent non-robust forward criteria}

The existence of criteria in auxiliary markets giving rise to equivalent behaviour can, in fact, be established in far greater generality. Indeed, given the existence of a saddle-point, it follows immediately that the robust forward criterion consisting of the pair $U(x, t)$ in (4.39) and $\gamma_{t, T}$ in (4.23) ranks investment strategies in the same way as does the (non-robust) forward criterion defined via

$$
\tilde{U}(x, t):=U(x, t)+\int_{0}^{t} g_{s}\left(\bar{\eta}_{s}\right) d s,
$$


considered in the auxiliary market ${ }^{9}$ with the market price of risk $\left(\bar{\lambda}_{t}\right)$ in $(4.41)$.

In consequence, if there exists an optimal strategy for a given robust forward criterion, then that strategy is optimal also for a specific standard (non-robust) forward criterion in the reference market. This holds since for any two markets defined with respect to equivalent measures $\overline{\mathbb{Q}}$ and $\hat{\mathbb{P}}$, there is a one-to-one correspondence between forward criteria defined in the respective markets (modulo integrability conditions): $U\left(X_{t}^{\pi}, t\right)$ is a $\overline{\mathbb{Q}}$-(super)martingale if and only if $D_{t}^{\bar{\eta}} U\left(X_{t}^{\pi}, t\right)$ is a $\hat{\mathbb{P}}$-(super)martingale. The optimal strategies resulting from considering the criteria $U(x, t)$ in the $\overline{\mathbb{Q}}$-market and $D_{t}^{\bar{\eta}} U(x, t)$ in the $\hat{\mathbb{P}}$-market coincide. To summarise, if a robust forward criterion $U(x, t)$ and $g_{t}(\cdot)$ admits a saddle point then the optimal behaviour associated with this criterion is also optimal for the forward criterion (4.43) considered in the market with market price of risk $\lambda_{t}+\bar{\eta}_{t}^{1}$. Consequently, it is also optimal for the forward criterion

$$
D_{t}^{\bar{\eta}} \tilde{U}(x, t)=D_{t}^{\bar{\eta}}\left(U(x, t)+\int_{0}^{t} g_{s}\left(\bar{\eta}_{s}\right) d s\right)
$$

specified in the reference market ${ }^{10}$. This equivalent forward criterion is, of course, defined in terms of the optimal $\bar{\eta}_{t}$, which is part of the solution to the robust problem and not a priori known. Nevertheless, on a more abstract level this implies that viewed as a class of preference criteria, forward criteria can be argued to be 'closed' under the introduction of a certain type of model uncertainty. For a similar conclusion in terms of the use of different numeraires, see Theorem 2.5 in [39] or Section 5.1 in [38]. This should also be compared to [115], where it was shown that to invest with respect to a given stochastic differential utility combined with a certain model uncertainty is equivalent to considering a modified stochastic differential utility within the reference model (therein, entropic penalty functions were considered but for a Brownian filtration and under some additional boundedness assumptions, the results can be extended also to variational preferences). For stochastic differential utilities as well as for forward criteria, the

\footnotetext{
${ }^{9}$ Note that in case there exists a measure $\overline{\mathbb{Q}} \sim \mathbb{P}$ such that $\left.\mathbb{Q}\right|_{\mathcal{F}_{T}}$ coincides with the optimal measure (equivalently the optimum to the dual problem) at $T$ for all $T>0$, then the model $\overline{\mathbb{Q}}$, defines itself an arbitrage-free market featuring the same set of admissible strategies as are available in the $\mathbb{P}$-market. Consequently, the above conclusions can be expressed in terms of $\overline{\mathbb{Q}}$ rather than the auxiliary market price of risk $\lambda_{t}+\bar{\eta}_{t}^{1}, t \geq 0$.

Also note that, in principle, the same argument goes through also for more general types of penalty functions and, thus, for more general (non-Brownian) market models. Indeed, in case the measure satisfies the above conditions, it holds on any finite interval $[0, T)$ that the optimal strategy associated with a robust criterion $U(x, t)$ and $\gamma$, also is optimal for the problem of maximizing utility with respect to the utility function $U(x, T)$, set at $T$ in the market specified by that associated optimal measure. However, in order to specify the associated non-robust criterion for all times $t \geq 0$, a few issues arise. Indeed, for more general penalty-function, it is no longer possible to use $t=0$ as a reference point in the way done in (4.43), but a criterion of the type
}

$$
\tilde{U}(x, t)=U(x, t)-\gamma_{t, \infty}(\overline{\mathbb{Q}}),
$$

would need to be considered. Hence, in order to avoid the difficulties associated with defining $\gamma_{t, \infty}(\cdot)$ we refrain to discuss the relation to non-robust criteria within the Brownian context.

${ }^{10}$ For a fixed terminal horizon $T>0$, the above argument simply amounts to the fact that, according to Bayes' rule, it holds that

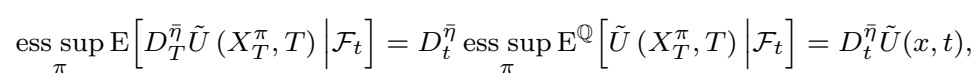

where, in particular, the supremum on the left and the right hand sides are attained for the same optimal strategy. 
underlying reason is that the notion is general enough to allow for stochastic preferences. In particular, use of deterministic utility functions under model uncertainty is, under some conditions, equivalent to the use of a specific stochastic utility in a fixed model. A fact which might be used to motivate the use of stochastic utility functions (cf. the use of different numeraires).

It follows that formally, the preferences corresponding to the robust criteria considered in this chapter may be embedded within the class of forward criteria. Nevertheless, we believe the example studied in this section illustrates that the notion of robust forward criteria is of great interest. The aim of these criteria and the associated specific modelling of model uncertainty, is to disentangle the impact of the preferences originating from risk and model-ambiguity, respectively. A related and more involved question is under what conditions a given (volatile non-robust) forward criterion can be written as a non-volatile robust forward criterion with respect to some non-trivial penalty function. This question is left for future research. We also remark that the analysis in this chapter and, thus, the above discussion, is restricted to measures equivalent to $\mathbb{P}$. Considering absolutely continuous measures introduces further complexity (cf. [110] for the static case) but should not alter the main conclusions.

\subsubsection{Further remarks on the volatility of the equivalent forward cri- teria}

We close this section with some remarks on the volatility of the equivalent criterion specified in (4.44). Recall that [95] established (cf. Theorem 4 and the discussion on p. 8 therein) that for any $\eta \in \bigcap_{T} \mathcal{Q}_{0, T}$ and deterministic solution $u: \mathbb{R}_{+} \times[0, \infty) \rightarrow \mathbb{R}$ to the equation

$$
u_{t}=\frac{1}{2} \frac{u_{x}^{2}}{u_{x x}}, \quad t \geq 0
$$

it holds that

$$
U(x, t)=D_{t}^{\eta} u\left(x, \int_{0}^{t}\left(\lambda_{s}+\eta_{s}\right)^{2} d s\right)
$$

is a forward criterion in the reference market with market price of risk $\lambda$. It follows from our discussion above that, for any solution to (4.45), the random field

$$
u\left(x, \int_{0}^{t} \tilde{\lambda}_{s}^{2} d s\right)
$$

is a forward criterion in the market with market price of risk $\tilde{\lambda}_{t}$ (see [96]). Since $u(x, t)=\ln x-t / 2$ is a solution to (4.45), the criterion in (4.42) is of this type for the specific choice of $\bar{\eta}$. However, as noted above, in general it is not clear whether criteria of the form (4.46) correspond to robust forward criteria for some non-trivial penalty function. 
These remarks illustrate that the assumption of non-volatility of the criterion is market specific. A straightforward calculation yields that the dynamics of the criterion in (4.46) are given by

$$
d U(x, t)=\frac{1}{2} \frac{\left(U_{x}(x, t) \lambda_{t}+U_{x}(x, t) \eta_{t}\right)^{2}}{U_{x x}(x, t)} d t+U(x, t) \eta_{t} d W_{t}
$$

Its volatility is given by $a(x, t)=U(x, t) \eta_{t}=D_{t}^{\eta} u\left(x, \int_{0}^{t}\left(\lambda_{s}+\eta_{s}\right)^{2} d s\right) \eta_{t}$, in particular $U$ satisfies the SPDE (4.28) for $t \geq 0$ with respect to this choice. Hence, while the criterion given in (4.47) is non-volatile, the corresponding criterion in the reference market is volatile.

Similarly, while the criterion in (4.39) is non-volatile, the equivalent (non-robust) criterion in the reference market is volatile. Indeed, the non-volatility requirement is placed on the random field associated with the robust criterion. Consequently, while these criteria will be non-volatile in the market specified by the optimal measure (in case a saddle-point exists), they correspond to specific volatile criteria in the reference market. This should be compared to the criterion (4.40) which is non-volatile in the reference market itself.

\subsection{Proofs}

\subsubsection{Proof of Theorem 4.6}

The aim of Section 4.6.1 is to prove Theorem 4.6. Note that for $t=0$, the assumptions of Theorem 4.6 differ from the assumptions in [110], however, the results are similar in style. Moreover, given that the conjugacy relations holds for $t=0$, the conditional conjugacy relations are expected to hold as well. In order to verify that this is the case, we follow the same procedure as in [129] where a conditional conjugacy relation for the non-robust case was proven. To that end, contrary to the claim, we assume that the conditional conjugacy does not hold (cf. pp. 122). Then, by taking expectations we reduce the problem to a $\mathcal{F}_{0}$-measurable conjugacy relation, for which we prove equality by use of arguments similar to the ones used in [110] (Proposition 4.22 and Corollary 4.23). Hence, we obtain a contradiction and conclude. Auxiliary results and details are worked out below.

\section{Notation and auxiliary results}

For the proof of Theorem 4.6, fix two time-points $0 \leq t \leq T<\infty$ and a random variable $\kappa \in L_{+}^{\infty}\left(\mathcal{F}_{t}\right)$. We will typically consider $\kappa=\mathbb{1}_{A}, A \in \mathcal{F}_{t}$, and use it to localise arguments to a set. Without further notice, the notation $Z_{t, T} \in \mathcal{Z}_{T}^{a}$ will be used to denote an element of the set $\left\{Z_{t, T} \mid Z \in \mathcal{Z}_{T}^{a}\right\}$. We also write $Z \in \mathcal{Q}_{t, T}$ to denote an element of the set $\left\{Z^{\mathbb{Q}} \mid \mathbb{Q} \in \mathcal{Q}_{t, T}\right\}$. 
Unless stated otherwise, all the $L^{p}$-spaces (and their duals), $p \in[0, \infty]$, will be with respect to $\left(\Omega, \mathcal{F}_{T},\left.\mathbb{P}\right|_{\mathcal{F}_{T}}\right)$.

Let $\mathcal{K}_{t, T}:=\left\{\int_{t}^{T} \pi_{s} d S_{s}: \pi \in \mathcal{A}_{b d}\right\}$ and $\mathcal{C}_{t, T}:=\left(\mathcal{K}_{t, T}-L_{+}^{0}\right) \cap L^{\infty}$. Note that the optimization over $\mathcal{K}_{t, T}$ in (4.2), might be replaced by optimization over $\mathcal{C}_{t, T}$. Then, for $\mathbb{Q} \in \mathcal{Q}_{t, T}$, introduce the function

$$
u_{\kappa}^{\mathbb{Q}}(\xi)=\sup _{g \in \mathcal{C}_{t, T}} \mathrm{E}\left[\kappa Z_{t, T}^{\mathbb{Q}} U(\xi+g, T)\right], \quad \xi \in L^{\infty}\left(\mathcal{F}_{t}\right) .
$$

Next, let $\mathcal{D}_{t, T}:=\left\{\zeta^{*} \in\left(L^{\infty}\right)^{*}:\left\langle\zeta^{*}, \zeta\right\rangle \leq 0\right.$ for all $\left.\zeta \in \mathcal{C}_{t, T}\right\}$ and for $\eta \in L_{+}^{1}\left(\mathcal{F}_{t}\right)$, let $\mathcal{D}_{t, T}^{\eta}:=$ $\left\{\zeta^{*} \in \mathcal{D}_{t, T}:\left\langle\zeta^{*}, \xi\right\rangle=\langle\eta, \xi\rangle\right.$, for all $\left.\xi \in L^{\infty}\left(\mathcal{F}_{t}\right)\right\}$. Then, introduce the function $\mathbb{V}_{\kappa}^{\mathbb{Q}}: \mathcal{D}_{t, T} \rightarrow$ $(-\infty, \infty]$ via

$$
\mathbb{V}_{\kappa}^{\mathbb{Q}}\left(\zeta^{*}\right):= \begin{cases}\mathrm{E}\left[\kappa Z_{t, T}^{\mathbb{Q}} V\left(\zeta^{*} /\left(\kappa Z_{t, T}^{\mathbb{Q}}\right), T\right)\right], & \zeta^{*} \in L_{+}^{1} \text { and }\left\{\zeta^{*}>0\right\} \subseteq\{\kappa>0\} \\ \infty, & \text { otherwise. }\end{cases}
$$

and the function $v_{\kappa}^{\mathbb{Q}}: L^{1}\left(\mathcal{F}_{t}\right) \rightarrow(-\infty, \infty]$ via

$$
v_{\kappa}^{\mathbb{Q}}(\eta):= \begin{cases}\inf _{\zeta^{*} \in \mathcal{D}_{t, T}^{\eta}} \mathbb{V}_{\kappa}^{\mathbb{Q}}\left(\zeta^{*}\right), & \eta \in L_{+}^{1}\left(\mathcal{F}_{t}\right), \\ \infty, & \eta \in L^{1}\left(\mathcal{F}_{t}\right) \backslash L_{+}^{1}\left(\mathcal{F}_{t}\right) .\end{cases}
$$

For ease of reference, we next present Lemma A.4 from [129]. Note that the proof of this result exploits that the market satisfies NFLVR on finite horizons.

Lemma 4.19. A random variable $\zeta^{*}$ is in $\mathcal{D}_{t, T} \cap L_{+}^{1}$, if and only if, there exists $\eta \in L_{+}^{1}\left(\mathcal{F}_{t}\right)$ and $Z_{t, T} \in \mathcal{Z}_{T}^{a}$ such that $\zeta^{*}=\eta Z_{t, T}$.

The next result follows by applying Propositions A1 and A3 in [129] to the auxiliary stochastic utility function $\tilde{U}(x, T)$ in (4.5).

Proposition 4.20. Let $\mathbb{Q} \in \mathcal{Q}_{t, T}$ and $\kappa \in L_{+}^{\infty}\left(\mathcal{F}_{t}\right)$ such that $\kappa Z_{t, T}^{\mathbb{Q}} U(x, T) \in L^{1}, x \in \mathbb{R}$, and $\tilde{U}(x, T):=\mathbb{1}_{\kappa=0} U(x, T)+\mathbb{1}_{\kappa>0} Z_{t, T}^{\mathbb{Q}} U(x, T), x \in \mathbb{R}$, (cf. (4.5)) satisfies the non-singularity condition 3.3 in [129]. Then, for any $\xi \in L^{\infty}\left(\mathcal{F}_{t}\right)$,

$$
u_{\kappa}^{\mathbb{Q}}(\xi)=\inf _{\eta \in L^{1}\left(\mathcal{F}_{t}\right)}\left(v_{\kappa}^{\mathbb{Q}}(\eta)+\langle\xi, \eta\rangle\right) .
$$

Proof. As $\mathbb{Q} \sim \mathbb{P}, Z_{t, T}^{\mathbb{Q}}>0$, $\mathbb{P}$-a.s. Moreover, due to assumption $\tilde{U}(x, T), x \in \mathbb{R}$, is integrable and satisfies the non-singularity condition. Proposition A1 in [129] can therefore be applied to the auxiliary random utility function ${ }^{11} \tilde{U}(x, T), x \in \mathbb{R}$. This yields,

$$
u_{\kappa}^{\mathbb{Q}}(\xi)=\inf _{\zeta^{*} \in \mathcal{D}_{t, T}}\left(\mathbb{V}_{\kappa}^{\mathbb{Q}}\left(\zeta^{*}\right)+\left\langle\zeta^{*}, \xi\right\rangle\right) .
$$

\footnotetext{
${ }^{11}$ Note that although $Z_{t, T}^{\mathbb{Q}} U(x, T) \in L^{1}$, it is not a priori clear whether $Z_{t, s}^{\mathbb{Q}} U(s, x) \in L^{1}\left(\mathcal{F}_{s}\right)$, for $t<s<T$. Hence, it is not clear whether the associated random field is actually a utility field in the sense of Definition 4.1 (the field could easily be adjusted in order for the utility and path regularity conditions to hold). However, Proposition A1 in [129] only makes use of the slice $U(x, T)$ and can, thus, be applied under the given assumptions.
} 
Indeed, on $\{\kappa>0\}, \tilde{U}(x, T)=Z_{t, T}^{\mathbb{Q}} U(x, T)$ and $\tilde{V}(y, T)=Z_{t, T}^{\mathbb{Q}} V\left(y / Z_{t, T}^{\mathbb{Q}}, T\right)$. Moreover, according to Proposition A3 in [129], their definition of $\mathbb{V}_{\kappa}$ with respect to $\tilde{V}(y, t)$ (valid for all $\left.\zeta^{*} \in\left(L^{\infty}\right)^{*}\right)$ coincides with the definition of $\mathbb{V}_{\kappa}^{\mathbb{Q}}$ in (4.48) for all $\zeta^{*} \in \mathcal{D}_{t, T}$.

The definition of $\mathbb{V}_{\kappa}^{\mathbb{Q}}$ (cf. (4.48)) implies that the set $\mathcal{D}_{t, T}$ in (4.50) can be replaced by $\mathcal{D}_{t, T} \cap L_{+}^{1}$. According to Lemma 4.19, for every $\zeta^{*} \in \mathcal{D}_{t, T} \cap L_{+}^{1}$, there exists $\eta \in L_{+}^{1}\left(\mathcal{F}_{t}\right)$ and $Z_{t, T} \in \mathcal{Z}_{T}^{a}$ such that $\zeta^{*}=\eta Z_{t, T}$. Since $\mathrm{E}\left[Z_{t, T} \mid \mathcal{F}_{t}\right]=1$, a.s., it follows that

$$
\left\langle\zeta^{*}, \xi\right\rangle=\mathrm{E}\left[\eta Z_{t, T} \xi\right]=\mathrm{E}[\eta \xi]=\langle\eta, \xi\rangle, \quad \text { for all } \xi \in L^{\infty}\left(\mathcal{F}_{t}\right)
$$

That is to say, for every $\zeta^{*} \in \mathcal{D}_{t, T} \cap L_{+}^{1}$, there exists $\eta \in L_{+}^{1}\left(\mathcal{F}_{t}\right)$ such that $\zeta^{*} \in \mathcal{D}_{t, T}^{\eta}$. Conversely, every $\zeta^{*} \in \mathcal{D}_{t, T}^{\eta}$, for $\eta \in L_{+}^{1}$, is in $\mathcal{D}_{t, T}$. Hence, it follows from (4.50) that

$$
\begin{aligned}
u_{\kappa}^{\mathbb{Q}}(\xi) & =\inf _{\eta \in L_{+}^{1}\left(\mathcal{F}_{t}\right)} \inf _{\zeta^{*} \in \mathcal{D}_{t, T}^{\eta}}\left(\mathbb{V}_{\kappa}^{\mathbb{Q}}\left(\zeta^{*}\right)+\langle\eta, \xi\rangle\right) \\
& =\inf _{\eta \in L_{+}^{1}\left(\mathcal{F}_{t}\right)}\left(v_{\kappa}^{\mathbb{Q}}(\eta)+\langle\xi, \eta\rangle\right) .
\end{aligned}
$$

Since $v_{\kappa}^{\mathbb{Q}}(\eta)=\infty$ for $\eta \in L^{1}\left(\mathcal{F}_{t}\right) \backslash L_{+}^{1}\left(\mathcal{F}_{t}\right)$, the infimum might equally well be taken over $L^{1}\left(\mathcal{F}_{t}\right)$ and the result follows.

The next result is this setting's analogue of Lemma 4.6 in [110]. It is proven by use of the same lopsided minimax theorem as is used therein. Together with Lemma 4.20, it is the cornerstone of the proof of the duality relation in Proposition 4.22 below.

Lemma 4.21. Assume that $\mathcal{Q}_{t, T}$ is weakly compact and that $U(x, t)$ and $\gamma$ satisfy Assumption 4.5. Then,

$$
\sup _{g \in \mathcal{C}_{t, T}} \inf _{Z \in \mathcal{Q}_{t, T}} \mathrm{E}\left[\kappa\left(Z U(\xi+g, T)+\gamma_{t, T}(Z)\right)\right]=\inf _{Z \in \mathcal{Q}_{t, T}} \sup _{g \in \mathcal{C}_{t, T}} \mathrm{E}\left[\kappa\left(Z U(\xi+g, T)+\gamma_{t, T}(Z)\right)\right] .
$$

Proof. For given $\xi \in L^{\infty}\left(\mathcal{F}_{t}\right)$ and $g \in \mathcal{C}_{t, T}$ there exists $a>0$ such that $\xi+g \geq-a$ a.s. Hence, $U(\xi+g, T) \geq U(-a, T)$. For a sequence $\left(Z_{n}\right)_{n \in \mathbb{N}}, Z_{n} \in \mathcal{Q}_{t, T}$, such that $Z_{n} \rightarrow Z$ a.s., we might thus use Fatou's Lemma to obtain

$$
\liminf _{n \rightarrow \infty} \mathrm{E}\left[\kappa Z_{n}\left(U(\xi+g, T)+U^{-}(-a, T)\right)\right] \geq \mathrm{E}\left[\kappa Z\left(U(\xi+g, T)+U^{-}(-a, T)\right)\right] .
$$

As $\left\{Z_{n} U^{-}(-a, T)\right\}, Z_{n} \in \mathcal{Q}_{t, T}$, is UI due to Assumption 4.5, it follows that

$$
\lim _{n \rightarrow \infty} \mathrm{E}\left[\kappa Z_{n} U^{-}(-a, T)\right]=\mathrm{E}\left[\kappa Z U^{-}(-a, T)\right],
$$

and, thus, (4.52) implies that the function $Z \rightarrow \mathrm{E}[\kappa Z U(\xi+g, T)]$ is lower semicontinuous with respect to a.s.-convergence on $\mathcal{Q}_{t, T}$. As $\mathcal{Q}_{t, T}$ is convex and weakly compact, it is UI. Hence, 
$Z \rightarrow \mathrm{E}[\kappa Z U(\xi+g, T)]$ is lower semicontinuious also with respect to convergence in $L^{1}$. This, in turn, yields weak lower semicontinuity as the function is convex (affine).

According to Definition $4.2, Z \rightarrow \mathrm{E}\left[\kappa \gamma_{t, T}(Z)\right]$ is also convex and weakly lower semi-continuous on $\mathcal{Q}_{t, T}$, which is convex and weakly compact due to Assumption 4.5. On the other hand, for each $Z \in \mathcal{Q}_{t, T}, g \rightarrow \mathrm{E}[\kappa Z U(\xi+g)]$ is a concave functional defined on the convex set $\mathcal{C}_{t, T}$. Hence, we might apply the Lopsided minimax theorem (cf. Chapter 6 in [7]) to obtain the desired result.

Next, we introduce the function $u_{\kappa}: L^{\infty}\left(\mathcal{F}_{t}\right) \rightarrow(-\infty, \infty]$ by

$$
u_{\kappa}(\xi)=\sup _{g \in \mathcal{C}_{t, T}} \inf _{\mathbb{Q} \in \mathcal{Q}_{t, T}} \mathrm{E}\left[\kappa\left(Z_{t, T}^{\mathbb{Q}} U(\xi+g, T)+\gamma_{t, T}(\mathbb{Q})\right)\right]
$$

and the function $v_{\kappa}: L^{1}\left(\mathcal{F}_{t}\right) \rightarrow(-\infty, \infty]$ via

$$
v_{\kappa}(\eta)=\inf _{\mathbb{Q} \in \mathcal{Q}_{t, T}}\left(v_{\kappa}^{\mathbb{Q}}(\eta)+\mathrm{E}\left[\kappa \gamma_{t, T}(\mathbb{Q})\right]\right) .
$$

The next result establishes the conjugacy relations between $u_{\kappa}$ and $v_{\kappa}$. This is the key result upon which the proof on the conditional versions in Theorem 4.6 relies.

Proposition 4.22. Assume that $\mathcal{Q}_{t, T}$ is weakly compact and that $U(x, t)$ and $\gamma$ satisfy Assumption 4.5. Then, for all $\xi \in L^{\infty}\left(\mathcal{F}_{t}\right)$ it holds that

$$
u_{\kappa}(\xi)=\inf _{\eta \in L^{1}\left(\mathcal{F}_{t}\right)}\left(v_{\kappa}(\eta)+\langle\xi, \eta\rangle\right) .
$$

Proof. By use of Lemma 4.21 we obtain

$$
\begin{aligned}
u_{\kappa}(\xi) & =\sup _{g \in \mathcal{C}_{t, T}} \inf _{\mathbb{Q} \in \mathcal{Q}_{t, T}} \mathrm{E}\left[\kappa\left(Z_{t, T}^{\mathbb{Q}} U(\xi+g, T)+\gamma_{t, T}(\mathbb{Q})\right)\right] \\
& =\inf _{\mathbb{Q} \in \mathcal{Q}_{t, T}}\left(\sup _{g \in \mathcal{C}_{t, T}} \mathrm{E}\left[\kappa Z_{t, T}^{\mathbb{Q}} U(\xi+g, T)\right]+\mathrm{E}\left[\kappa \gamma_{t, T}(\mathbb{Q})\right]\right) \\
& =\inf _{\mathbb{Q} \in \mathcal{Q}_{t, T}}\left(u_{\kappa}^{\mathbb{Q}}(\xi)+\mathrm{E}\left[\kappa \gamma_{t, T}(\mathbb{Q})\right]\right) .
\end{aligned}
$$

Note that if $U\left(x^{0}, T\right) \in L^{1}$, for some $x^{0} \in \mathbb{R}$, then $U(x, T) \in L^{1}$ for all $x \in \mathbb{R}$. Indeed, due to concavity it holds that

$$
\lambda \mathrm{E}[U(x, T)]+(1-\lambda) \mathrm{E}[U(y, T)] \leq \mathrm{E}\left[U\left(x^{0}, T\right)\right], \quad \text { for } x<x_{0}<y, \lambda x+(1-\lambda) y=x_{0} .
$$

Since $\mathbb{P} \in \mathcal{Q}_{t, T}$ and $U(x, T) \in L^{1}$ due to assumption, we might thus, without loss of generality, replace the set $\mathcal{Q}_{t, T}$ in (4.53) by

$$
\mathcal{Q}_{t, T}^{\kappa}:=\left\{\mathbb{Q} \in \mathcal{Q}_{t, T}: \kappa Z_{t, T}^{\mathbb{Q}} U(x, T) \in L^{1}\right\} .
$$


Due to Assumption 4.5, we might then apply Proposition 4.20 for each $\mathbb{Q} \in \mathcal{Q}_{t, T}^{\kappa}$ in order to obtain

$$
\begin{aligned}
u_{\kappa}(\xi) & =\inf _{\mathbb{Q} \in \mathcal{Q}_{t, T}^{\kappa}}\left(\inf _{\eta \in L^{1}\left(\mathcal{F}_{t}\right)}\left(v_{\kappa}^{\mathbb{Q}}(\eta)+\langle\xi, \eta\rangle\right)+\mathrm{E}\left[\kappa \gamma_{t, T}(\mathbb{Q})\right]\right) \\
& =\inf _{\eta \in L^{1}\left(\mathcal{F}_{t}\right)}\left(\inf _{\mathbb{Q} \in \mathcal{Q}_{t, T}^{\kappa}}\left(v_{\kappa}^{\mathbb{Q}}(\eta)+\mathrm{E}\left[\kappa \gamma_{t, T}(\mathbb{Q})\right]\right)+\langle\xi, \eta\rangle\right)=\inf _{\eta \in L^{1}\left(\mathcal{F}_{t}\right)}\left(v_{\kappa}(\eta)+\langle\xi, \eta\rangle\right),
\end{aligned}
$$

where it remains to argue the last step. To this end, note that for each $\zeta^{*} \in \mathcal{D}_{t, T}^{\eta}, \eta \in L^{1}\left(\mathcal{F}_{t}\right)$, it holds that

$$
\begin{aligned}
\mathrm{E}\left[\kappa Z_{t, T}^{\mathbb{Q}} V\left(\zeta^{*} / \kappa Z_{t, T}^{\mathbb{Q}}, T\right)\right]+\mathrm{E}[\xi \eta] & \geq \mathrm{E}\left[\kappa Z_{t, T}^{\mathbb{Q}}\left(U(\xi+g, T)-\zeta^{*}(\xi+g) / \kappa Z_{t, T}^{\mathbb{Q}}\right)\right]+\mathrm{E}[\xi \eta] \\
& =\mathrm{E}\left[\kappa Z_{t, T}^{\mathbb{Q}} U(\xi+g, T)\right]-\mathrm{E}\left[\zeta^{*}(\xi+g)\right]+\mathrm{E}[\xi \eta] \\
& \geq \mathrm{E}\left[\kappa Z_{t, T}^{\mathbb{Q}} U(\xi+g, T)\right] .
\end{aligned}
$$

Hence, it follows that $\mathcal{Q}_{t, T}^{\kappa}$ can be replaced by $\mathcal{Q}_{t, T}$ without loss of generality. This completes the proof.

Corollary 4.23. Under the same assumptions as in Proposition 4.22, it holds for all $\eta \in L_{+}^{1}\left(\mathcal{F}_{t}\right)$ that

$$
v_{\kappa}(\eta)=\sup _{\xi \in L^{\infty}\left(\mathcal{F}_{t}\right)}\left\{u_{\kappa}(\xi)-\langle\xi, \eta\rangle\right\}
$$

Proof. ${ }^{12}$ According to Proposition 4.20 (cf. Corollary A.2 in [129]), it holds that

$$
\inf _{\mathbb{Q} \in \mathcal{Q}_{t, T}^{\kappa}}\left(v_{\kappa}^{\mathbb{Q}}(\eta)+\mathrm{E}\left[\kappa \gamma_{t, T}(\mathbb{Q})\right]\right)=\inf _{\mathbb{Q} \in \mathcal{Q}_{t, T}^{\kappa}}\left(\sup _{\xi \in L^{\infty}\left(\mathcal{F}_{t}\right)}\left\{u_{\kappa}^{\mathbb{Q}}(\xi)-\mathrm{E}[\eta \xi]\right\}+\mathrm{E}\left[\kappa \gamma_{t, T}(\mathbb{Q})\right]\right),
$$

where $\mathcal{Q}_{t, T}^{\kappa}$ is as defined in (4.54). By use of the same arguments as used in the proof of Proposition 4.22 , it follows that $\mathcal{Q}_{t, T}^{\kappa}$ can be replaced by $\mathcal{Q}_{t, T}$ on both sides of the above equation. Hence, it follows that

$$
\begin{aligned}
v_{\kappa}(\eta) & =\inf _{\mathbb{Q} \in \mathcal{Q}_{t, T}}\left(\sup _{\xi \in L^{\infty}\left(\mathcal{F}_{t}\right)}\left\{u_{\kappa}^{\mathbb{Q}}(\xi)-\mathrm{E}[\eta \xi]\right\}+\mathrm{E}\left[\kappa \gamma_{t, T}(\mathbb{Q})\right]\right) \\
& =\sup _{\xi \in L^{\infty}\left(\mathcal{F}_{t}\right)}\left\{\inf _{\mathbb{Q} \in \mathcal{Q}_{t, T}}\left(u_{\kappa}^{\mathbb{Q}}(\xi)+\mathrm{E}\left[\kappa \gamma_{t, T}(\mathbb{Q})\right]\right)-\mathrm{E}[\eta \xi]\right\}=\sup _{\xi \in L^{\infty}\left(\mathcal{F}_{t}\right)}\left\{u_{\kappa}(\xi)-\mathrm{E}[\eta \xi]\right\},
\end{aligned}
$$

where the last step follows due to (4.53). It remains to justify the interchange of the infimum and supremum in the second step. As in the proof of Lemma 4.21, it follows that $Z \rightarrow \mathrm{E}[\kappa Z U(\xi+$ $g, T)], Z \in \mathcal{Q}_{t, T}$ is weakly lower semicontinuous. Hence, so is $\mathbb{Q} \rightarrow u_{\kappa}^{\mathbb{Q}}(\xi)$ as the supremum preserves lower semicontinuity. Moreover, $\xi \rightarrow u^{\mathbb{Q}}(\xi)-\mathrm{E}[\eta \xi]$ is concave. As $\mathcal{Q}_{t, T}$ is convex and weakly compact due to assumption and $L^{\infty}$ is convex, the same Lopsided minimax theorem as in the proof of Theorem 4.21 can be applied.

\footnotetext{
${ }^{12}$ According to Proposition $4.22, u_{\kappa}$ is the convex conjugate of $v_{\kappa}$. Hence, alternatively, in order to show that $v_{\kappa}$ is the convex conjugate of $u_{\kappa}$, it would be enough to show that $v_{\kappa}$ is convex and lower semi-continuous.
} 
In order to reduce the conditional conjugacy relations to the $\mathcal{F}_{0}$-measurable ones proven above, we establish yet an auxiliary result.

Lemma 4.24. For fixed $g \in \mathcal{C}_{t, T}$ and $\xi \in L^{\infty}\left(\mathcal{F}_{T}\right)$, it holds that

$$
\mathrm{E}\left[\underset{\mathbb{Q} \in \mathcal{Q}_{t, T}}{\operatorname{ess} \inf } \kappa\left(\mathrm{E}\left[Z_{t, T}^{\mathbb{Q}} U(\xi+g, T) \mid \mathcal{F}_{t}\right]+\gamma_{t, T}(\mathbb{Q})\right)\right]=\inf _{\mathbb{Q} \in \mathcal{Q}_{t, T}} \mathrm{E}\left[\kappa\left(Z_{t, T}^{\mathbb{Q}} U(\xi+g, T)+\gamma_{t, T}(\mathbb{Q})\right)\right] .
$$

Proof. The inequality ' $\leq$ ' is trivial. To show the reverse inequality, let

$$
J(\mathbb{Q}):=\kappa \mathrm{E}\left[Z_{t, T}^{\mathbb{Q}} U(\xi+g, T) \mid \mathcal{F}_{t}\right]+\kappa \gamma_{t, T}(\mathbb{Q}), \quad \mathbb{Q} \in \mathcal{Q}_{t, T} .
$$

Note that $\mathbb{P} \in \mathcal{Q}_{t, T}$. Moreover, since $U(x, T) \in L^{1}$ due to assumption, it holds that

$$
J(\mathbb{P})=\kappa \mathrm{E}\left[U(\xi+g, T) \mid \mathcal{F}_{t}\right]+\kappa \gamma_{t, T}(\mathbb{P}) \in L^{1}\left(\mathcal{F}_{t}\right) .
$$

Hence, w.l.o.g. the set $\mathcal{Q}_{t, T}$ on the left hand side of the statement in the Lemma can be replaced by $\tilde{\mathcal{Q}}_{t, T}:=\left\{\mathbb{Q} \in \mathcal{Q}_{t, T}: J(\mathbb{Q}) \in L^{1}\left(\mathcal{F}_{t}\right)\right\}$.

Next, we claim that the set $\left\{J(\mathbb{Q}) \mid \mathbb{Q} \in \tilde{\mathcal{Q}}_{t, T}\right\}$ is directed downwards. Indeed, let $\mathbb{Q}_{1}, \mathbb{Q}_{2} \in \tilde{\mathcal{Q}}_{t, T}$ and define $A:=\left\{J\left(\mathbb{Q}_{1}\right) \leq J\left(\mathbb{Q}_{2}\right)\right\} \in \mathcal{F}_{t}$. Let $\overline{\mathbb{Q}}$ be the measure defined with respect to $Z_{T}:=$ $\mathbb{1}_{A} Z_{T}^{\mathbb{Q}_{1}}+\mathbb{1}_{A^{c}} Z_{T}^{\mathbb{Q}_{2}}$. According to Lemma 3.3 in [43], $\overline{\mathbb{Q}} \in \mathcal{Q}_{t, T}$ and, furthermore,

$$
\begin{aligned}
J(\overline{\mathbb{Q}}) & =\kappa \mathrm{E}\left[\left(\mathbb{1}_{A} Z_{t, T}^{\mathbb{Q}_{1}}+\mathbb{1}_{A^{c}} Z_{t, T}^{\mathbb{Q}_{2}}\right) U(\xi+g, T) \mid \mathcal{F}_{t}\right]+\kappa \gamma_{t, T}(\overline{\mathbb{Q}}) \\
& =\kappa \mathbb{1}_{A}\left(\mathrm{E}^{\mathbb{Q}_{1}}\left[U(\xi+g, T) \mid \mathcal{F}_{t}\right]+\gamma_{t, T}\left(\mathbb{Q}_{1}\right)\right)+\kappa \mathbb{1}_{A^{c}}\left(\mathrm{E}^{\mathbb{Q}_{2}}\left[U(\xi+g, T) \mid \mathcal{F}_{t}\right]+\gamma_{t, t}\left(\mathbb{Q}_{2}\right)\right) \\
& =\mathbb{1}_{A} J\left(\mathbb{Q}_{1}\right)+\mathbb{1}_{A^{c}} J\left(\mathbb{Q}_{2}\right)=\min \left\{J\left(\mathbb{Q}_{1}\right), J\left(\mathbb{Q}_{2}\right)\right\} .
\end{aligned}
$$

In particular, this implies that $\overline{\mathbb{Q}} \in \tilde{\mathcal{Q}}_{t, T}$. Consequently, it also follows that $\left\{J(\mathbb{Q}) \mid \mathbb{Q} \in \tilde{\mathcal{Q}}_{t, T}\right\}$ is closed under minimization and so directed downwards. Hence, due to Neveu [100], there exists a sequence $\mathbb{Q}_{n} \in \tilde{\mathcal{Q}}_{t, T}$ such that $J\left(\mathbb{Q}_{n}\right)$ is decreasing and

$$
\underset{\mathbb{Q} \in \tilde{\mathcal{Q}}_{t, T}}{\operatorname{ess} \inf } J(\mathbb{Q})=\lim _{n \rightarrow \infty} J\left(\mathbb{Q}_{n}\right) .
$$

Use of the monotone convergence theorem then yields

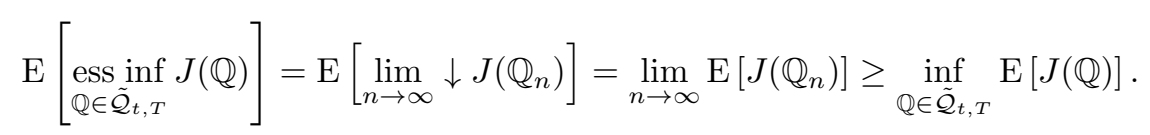

Combined with the above and using that $\tilde{\mathcal{Q}}_{t, T} \subseteq \mathcal{Q}_{t, T}$, we obtain

$$
\mathrm{E}\left[\operatorname{essinf}_{\mathbb{Q} \in \mathcal{Q}_{t, T}} J(\mathbb{Q})\right]=\mathrm{E}\left[\operatorname{ess}_{\mathbb{Q} \in \tilde{\mathcal{Q}}_{t, T}}^{\operatorname{enf}} J(\mathbb{Q})\right] \geq \inf _{\mathbb{Q} \in \tilde{\mathcal{Q}}_{t, T}} \mathrm{E}[J(\mathbb{Q})] \geq \inf _{\mathbb{Q} \in \mathcal{Q}_{t, T}} \mathrm{E}[J(\mathbb{Q})],
$$

and we easily conclude. 


\section{Proof of Theorem 4.6}

Proof of relation (4.6). First, we show that the inequality ' $\leq$ ' holds. To this end, note that for fixed $\bar{g} \in \mathcal{C}_{t, T}$ and $\overline{\mathbb{Q}} \in \mathcal{Q}_{t, T}$, it trivially holds that

$$
\underset{\mathbb{Q} \in \mathcal{Q}_{t, T}}{\operatorname{essinf}}\left(\mathrm{E}^{\mathbb{Q}}\left[U(\xi+\bar{g}, T) \mid \mathcal{F}_{t}\right]+\gamma_{t, T}(\mathbb{Q})\right) \leq \underset{g \in \mathcal{C}_{t, T}}{\operatorname{ess} \sup } \mathrm{E}^{\overline{\mathbb{Q}}}\left[U(\xi+g, T) \mid \mathcal{F}_{t}\right]+\gamma_{t, T}(\overline{\mathbb{Q}}),
$$

with $\xi \in L^{\infty}\left(\mathcal{F}_{t}\right)$ and $\eta \in L_{+}^{1}\left(\mathcal{F}_{t}\right)$. Thus, it, immediately, follows that

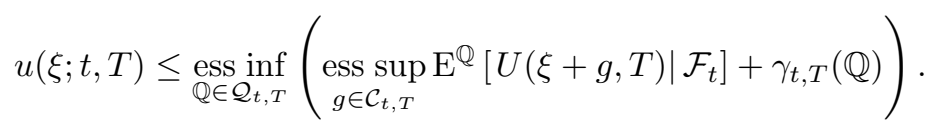

Next, for any $\mathbb{Q} \in \mathcal{M}_{T}^{a}, S$ is a local martingale and, thus, so is $\int_{0}^{t} \pi_{u} d S_{u}$, for all $\pi \in \mathcal{A}_{b d}$. Since, for all $\pi \in \mathcal{A}_{b d}$, there exists $a>0$ such that $\int_{0}^{t} \pi_{u} d S_{u}>-a, t \leq T$, it follows that $\mathrm{E}^{\mathbb{Q}}[g] \leq 0$, for all $g \in \mathcal{C}_{t, T}$. Since $U(x, T) \leq V(y, T)+x y$, for all $x \in \mathbb{R}, y \geq 0$, it, then, in turn, follows that for any $\mathbb{Q} \in \mathcal{Q}_{t, T}, Z_{t, T} \in \mathcal{Z}_{T}^{a}, \xi \in L^{\infty}\left(\mathcal{F}_{t}\right), g \in \mathcal{C}_{t, T}$ and $\eta \in L_{+}^{1}\left(\mathcal{F}_{t}\right)$;

$$
\begin{aligned}
\mathrm{E}^{\mathbb{Q}}\left[U(\xi+g, T) \mid \mathcal{F}_{t}\right] & \leq \mathrm{E}^{\mathbb{Q}}\left[V\left(\eta Z_{t, T} / Z_{t, T}^{\mathbb{Q}}, T\right) \mid \mathcal{F}_{t}\right]+\mathrm{E}\left[(\xi+g) \eta Z_{t, T} \mid \mathcal{F}_{t}\right] \\
& \leq \mathrm{E}^{\mathbb{Q}}\left[V\left(\eta Z_{t, T} / Z_{t, T}^{\mathbb{Q}}, T\right) \mid \mathcal{F}_{t}\right]+\xi \eta \quad \text { a.s. }
\end{aligned}
$$

In combination with $(4.55)$, this implies that

$$
\begin{aligned}
u(\xi ; t, T) & \leq \underset{\mathbb{Q} \in \mathcal{Q}_{t, T}}{\operatorname{essinf}}\left(\underset{Z \in \mathcal{Z}_{T}^{a}}{\operatorname{ess} \inf } \mathrm{E}^{\mathbb{Q}}\left[V\left(\eta Z_{t, T} / Z_{t, T}^{\mathbb{Q}}, T\right) \mid \mathcal{F}_{t}\right]+\xi \eta+\gamma_{t, T}(\mathbb{Q})\right) \\
& =v(\eta ; t, T)+\xi \eta
\end{aligned}
$$

for all $\eta \in L_{+}^{1}\left(\mathcal{F}_{t}\right)$, which completes the proof of the first inequality.

To prove the reverse inequality, we argue by contradiction and assume that there exist $\xi \in$ $L^{\infty}\left(\mathcal{F}_{t}\right), \varepsilon>0$ and $A \in \mathcal{F}_{t}$ such that

$$
\underset{\mathbb{Q} \in \mathcal{Q}_{t, T}}{\operatorname{essinf}}\left(\mathrm{E}^{\mathbb{Q}}\left[U(\xi+g, T) \mid \mathcal{F}_{t}\right]+\gamma_{t, T}(\mathbb{Q})\right)+\varepsilon \mathbb{1}_{A} \leq \mathrm{E}^{\mathbb{Q}}\left[V\left(\eta Z_{t, T} / Z_{t, T}^{\mathbb{Q}}, T\right) \mid \mathcal{F}_{t}\right]+\gamma_{t, T}(\mathbb{Q})+\xi \eta,
$$

for all $g \in \mathcal{K}_{t, T}, Z_{t, T} \in \mathcal{Z}_{T}^{a}, \mathbb{Q} \in \mathcal{Q}_{t, T}$ and $\eta \in L_{+}^{1}\left(\mathcal{F}_{t}\right)$. Note tat $u(\xi ; t, T)<\infty$ a.s. on $A$ and w.l.o.g. we assume that there exists $M<\infty$ such that $u(\xi ; t, T) \leq M$ a.s. on $A$. Next, multiplying the above inequality by $\kappa=\mathbb{1}_{A}$, noting that $\kappa=1 / \kappa$ on $A$ and taking expectations and using Lemma 4.24 we obtain

$\inf _{\mathbb{Q} \in \mathcal{Q}_{t, T}} \mathrm{E}\left[\kappa\left(Z_{t, T}^{\mathbb{Q}} U(\xi+g, T)+\gamma_{t, T}(\mathbb{Q})\right)\right]+\varepsilon P(A) \leq \mathrm{E}\left[\kappa Z_{t, T}^{\mathbb{Q}} V\left(\frac{\eta}{\kappa} \frac{Z_{t, T}}{Z_{t, T}^{\mathbb{Q}}}, T\right)\right]+\mathrm{E}\left[\kappa \gamma_{t, T}(\mathbb{Q})\right]+\mathrm{E}[\kappa \xi \eta]$,

for all $g \in \mathcal{K}_{t, T}, Z_{t, T} \in \mathcal{Z}_{T}^{a}, \mathbb{Q} \in \mathcal{Q}_{t, T}$ and $\eta \in L_{+}^{1}\left(\mathcal{F}_{t}\right)$; the expression in the first expectation on the right hand side is defined to be zero on $A^{c}$. Next, according to Lemma 4.19, for every $\zeta^{*} \in \mathcal{D}_{t, T}^{\eta} \cap L_{+}^{1}$, with $\eta \in L_{+}^{1}\left(\mathcal{F}_{t}\right)$, there exists $Z_{t, T} \in \mathcal{Z}_{T}^{a}$ such that

$$
\zeta^{*}=\eta Z_{t, T}
$$


By use of this fact and, moreover, taking the supremum over $g \in \mathcal{K}_{t, T}$, we obtain

$$
u_{\kappa}(\xi)+\varepsilon P(A) \leq \mathbb{V}_{\kappa}^{\mathbb{Q}}\left(\zeta^{*}\right)+\mathrm{E}\left[\kappa \gamma_{t, T}(\mathbb{Q})\right]+\langle\xi, \eta\rangle,
$$

for all $\eta \in L_{+}^{1}\left(\mathcal{F}_{t}\right)$ such that $\eta=\eta \mathbb{1}_{A}, \mathbb{Q} \in \mathcal{Q}_{t, T}$ and $\zeta^{*} \in \mathcal{D}_{t, T}^{\eta} \cap L_{+}^{1}$. Consequently, for any $\eta \in L_{+}^{1}\left(\mathcal{F}_{t}\right)$ and $\mathbb{Q} \in \mathcal{Q}_{t, T}$, the above inequality holds for all $\zeta^{*} \in \mathcal{D}_{t, T}^{\eta}$. Indeed, if $\zeta^{*} \notin L_{+}^{1}$ or if $\eta \neq \eta \mathbb{1}_{A}$, then it holds that $\mathbb{V}_{\kappa}^{\mathbb{Q}}\left(\zeta^{*}\right)=\infty$ (cf. (4.48)). Hence,

$$
u_{\kappa}(\xi)+\varepsilon P(A) \leq v_{\kappa}^{\mathbb{Q}}(\eta)+\mathrm{E}\left[\kappa \gamma_{t, T}(\mathbb{Q})\right]+\langle\xi, \eta\rangle,
$$

for all $\eta \in L_{+}^{1}\left(\mathcal{F}_{t}\right)$ and $\mathbb{Q} \in \mathcal{Q}_{t, T}$ and, thus, in turn, since $u_{\kappa}(\xi) \leq M<\infty$ (this is why we needed to introduce $\kappa$ ), we obtain

$$
u_{\kappa}(\xi)<u_{\kappa}(\xi)+\varepsilon P(A) \leq \inf _{\eta \in L^{1}\left(\mathcal{F}_{t}\right)}\left(v_{\kappa}(\eta)+\langle\xi, \eta\rangle\right) .
$$

According to Proposition 4.22 we have, thus, obtained a contradiction and we easily conclude.

Proof of relation (4.7). In particular, (4.6) implies that for all $\eta \in L^{1}\left(\mathcal{F}_{t}\right)$ and $\xi \in L^{\infty}\left(\mathcal{F}_{t}\right)$, it holds that $v(\eta ; t, T) \geq u(\xi ; t, T)-\xi \eta$. Hence, the inequality " $\geq "$ follows directly.

To prove the reverse inequality, we argue by contradiction and assume that there exist $\eta \in$ $L_{+}^{1}\left(\mathcal{F}_{t}\right), \varepsilon>0$ and $A \in \mathcal{F}_{t}$ such that

$$
\underset{\mathbb{Q} \in \mathcal{Q}_{t, T}}{\operatorname{ess} \inf }\left(\mathrm{E}^{\mathbb{Q}}\left[U(\xi+g, T) \mid \mathcal{F}_{t}\right]+\gamma_{t, T}(\mathbb{Q})\right)-\xi \eta+\varepsilon \mathbb{1}_{A} \leq \mathrm{E}^{\mathbb{Q}}\left[V\left(\eta Z_{t, T} / Z_{t, T}^{\mathbb{Q}}, T\right) \mid \mathcal{F}_{t}\right]+\gamma_{t, T}(\mathbb{Q}),
$$

for all $g \in \mathcal{K}_{t, T}, \xi \in L^{\infty}\left(\mathcal{F}_{t}\right), Z_{t, T} \in \mathcal{Z}_{T}^{a}$ and $\mathbb{Q} \in \mathcal{Q}_{t, T}$. Since $\eta$ might be replaced by $\eta \mathbb{1}_{A}$ without violating the above inequality, we assume without loss of generality that $\eta=0$ on $A^{c}$. Next, multiplying the above inequality by $\kappa=\mathbb{1}_{A}$, taking the expectation and using Lemma 4.24 yields

$\inf _{\mathbb{Q} \in \mathcal{Q}_{t, T}} \mathrm{E}\left[\kappa\left(Z_{t, T}^{\mathbb{Q}} U(\xi+g, T)+\gamma_{t, T}(\mathbb{Q})\right)\right]-\mathrm{E}[\xi \eta]+\varepsilon P(A) \leq \mathrm{E}\left[\kappa Z_{t, T}^{\mathbb{Q}} V\left(\frac{\eta}{\kappa} \frac{Z_{t, T}}{Z_{t, T}^{\mathbb{Q}}}, T\right)\right]+\mathrm{E}\left[\kappa \gamma_{t, T}(\mathbb{Q})\right]$, for all $g \in \mathcal{K}_{t, T}, \xi \in L^{\infty}\left(\mathcal{F}_{t}\right), \mathbb{Q} \in \mathcal{Q}_{t, T}$ and $Z_{t, T} \in \mathcal{Z}_{T}^{a}$. Next, according to Lemma 4.19, for every $\zeta^{*} \in \mathcal{D}_{t, T}^{\eta} \cap L_{+}^{1}$, there exists $Z_{t, T} \in \mathcal{Z}_{T}^{a}$ such that $\zeta^{*}=\eta Z_{t, T}$. Hence, it follows that

$$
u_{\kappa}(\xi)-\langle\xi, \eta\rangle+\varepsilon P(A) \leq \mathbb{V}_{\kappa}^{\mathbb{Q}}\left(\zeta^{*}\right)+\mathrm{E}\left[\gamma_{t, T}(\mathbb{Q})\right],
$$

for all $\xi \in L^{\infty}\left(\mathcal{F}_{t}\right), \mathbb{Q} \in \mathcal{Q}_{t, T}$ and $\zeta^{*} \in \mathcal{D}_{t, T}^{\eta} \cap L_{+}^{1}$. Since $\mathbb{V}_{\kappa}^{\mathbb{Q}}\left(\zeta^{*}\right)=\infty$, for any other $\zeta^{*} \in \mathcal{D}_{t, T}^{\eta}$, the above inequality holds for for all $\xi \in L^{\infty}\left(\mathcal{F}_{t}\right), \mathbb{Q} \in \mathcal{Q}_{t, T}$ and $\zeta^{*} \in \mathcal{D}_{t, T}^{\eta}$. Hence, we obtain

$$
u_{\kappa}(\xi)-\langle\xi, \eta\rangle+\varepsilon P(A) \leq v_{\kappa}^{\mathbb{Q}}(\eta)+\mathrm{E}\left[\gamma_{t, T}(\mathbb{Q})\right],
$$

for all $\xi \in L^{\infty}\left(\mathcal{F}_{t}\right)$ and $\mathbb{Q} \in \mathcal{Q}_{t, T}$ and, thus, in turn

$$
\sup _{\xi \in L^{\infty}}\left(u_{\kappa}(\xi)-\langle\xi, \eta\rangle\right)<\sup _{\xi \in L^{\infty}}\left(u_{\kappa}(\xi)-\langle\xi, \eta\rangle\right)+\varepsilon P(A) \leq v_{\kappa}(\eta),
$$


where was used that $\sup _{\xi \in L^{\infty}}\left(u_{\kappa}(\xi)-\langle\xi, \eta\rangle\right)<\infty .{ }^{13}$ According to Corollary 4.23 we have, thus, obtained a contradiction and we easily conclude.

\subsubsection{Proof of Lemmas 4.11 and 4.14}

Proof of Lemma 4.11. Fix $0<s<t<T<\infty$. Let $Z \in \mathcal{Z}_{t}^{a}$ and $\mathbb{Q} \in \mathcal{Q}_{s, t}$ and denote by $Z^{*}$ and $\mathbb{Q}^{*}$ the optimal elements in $\mathcal{Z}_{T}^{a}$ and $\mathcal{Q}_{t, T}$, respectively, for which $v\left(\eta Z_{s, t} / Z_{s, t}^{\mathbb{Q}} ; t, T\right)$ is attained. Then, it holds that

$$
\begin{aligned}
\mathrm{E}\left[Z _ { s , t } ^ { \mathbb { Q } } v \left(\eta \frac{Z_{s, t}}{Z_{s, t}^{\mathbb{Q}}} ;\right.\right. & \left.t, T) \mid \mathcal{F}_{s}\right]+\gamma_{s, t}(\mathbb{Q}) \\
& =\mathrm{E}\left[Z_{s, t}^{\mathbb{Q}}\left(\mathrm{E}\left[Z_{t, T}^{\mathbb{Q}^{*}} V\left(\eta \frac{Z_{s, t}}{Z_{s, t}^{\mathbb{Q}}} \frac{Z_{t, T}^{*}}{Z_{t, T}^{\mathbb{Q}^{*}}}, T\right) \mid \mathcal{F}_{t}\right]+\gamma_{t, T}\left(Z_{t, T}^{\mathbb{Q}^{*}}\right)\right) \mid \mathcal{F}_{s}\right]+\gamma_{s, t}(\mathbb{Q}) \\
& =\mathrm{E}\left[Z_{s, t}^{\mathbb{Q}} Z_{t, T}^{\mathbb{Q}^{*}} V\left(\eta \frac{Z_{s, t} Z_{t, T}^{*}}{Z_{s, t}^{\mathbb{Q}} Z_{t, T}^{\mathbb{Q}^{*}}}, T\right) \mid \mathcal{F}_{s}\right]+\gamma_{s, T}\left(Z_{s, t}^{\mathbb{Q}} Z_{t, T}^{\mathbb{Q}^{*}}\right) \\
& \geq \operatorname{ess}_{Z \in \mathcal{Z}_{T}^{a}}^{\operatorname{ess} \inf }\left\{\mathrm{E}\left[Z_{s, T}^{\mathbb{Q}} V\left(\eta \frac{Z_{s, T}}{Z_{s, T}^{\mathbb{Q}}}, T\right) \mid \mathcal{F}_{s}\right]+\gamma_{s, T}\left(Z_{s, T}^{\mathbb{Q}}\right)\right\}=v(\eta ; s, T),
\end{aligned}
$$

where the fact that $Z_{t} Z_{t, T}^{*} \in \mathcal{Z}_{T}^{a}$ and $\overline{\mathbb{Q}} \in \mathcal{Q}_{s, T}$ with $\frac{\mathrm{d} \overline{\mathbb{Q}}}{\left.\mathrm{d} \mathbb{|}\right|_{T}}=Z_{t}^{\mathbb{Q}} Z_{t, T}^{\mathbb{Q}^{*}}$, follow from the assumptions in the same way as in the proof of "i) implies iii)" of Proposition 4.14. ${ }^{14}$

Next, let $Z \in \mathcal{Z}_{T}^{a}$ and $\mathbb{Q} \in \mathcal{Q}_{0, T}$ be the optimal processes for which $v(\eta ; s, T)$ attains its infimum.

Then, it follows that

$$
\begin{aligned}
v(\eta ; s, T)= & \mathrm{E}\left[Z_{s, T}^{\mathbb{Q}} V\left(\eta \frac{Z_{s, T}}{Z_{s, T}^{\mathbb{Q}}}, T\right) \mid \mathcal{F}_{s}\right]+\gamma_{s, T}\left(Z_{s, T}^{\mathbb{Q}}\right) \\
& =\mathrm{E}\left[Z_{s, t}^{\mathbb{Q}}\left(\mathrm{E}\left[Z_{t, T}^{\mathbb{Q}} V\left(\eta \frac{Z_{s, t}}{Z_{s, t}^{\mathbb{Q}}} \frac{Z_{t, T}}{Z_{t, T}^{\mathbb{Q}}}, T\right) \mid \mathcal{F}_{t}\right]+\gamma_{t, T}\left(Z_{t, T}^{\mathbb{Q}}\right)\right) \mid \mathcal{F}_{s}\right]+\gamma_{s, t}(\mathbb{Q}) \\
& \geq \mathrm{E}\left[Z_{s, t}^{\mathbb{Q}} v\left(\eta \frac{Z_{s, t}}{Z_{s, t}^{\mathbb{Q}}} ; t, T\right) \mid \mathcal{F}_{s}\right]+\gamma_{s, t}(\mathbb{Q}) \geq v(\eta ; s, T),
\end{aligned}
$$

where the inequalities follow from the fact that $\mathbb{Q} \in \mathcal{Q}_{0, T}$ implies $\mathbb{Q} \in \mathcal{Q}_{t, T}$ and $\left.\mathbb{Q}\right|_{\mathcal{F}_{t}} \in \mathcal{Q}_{s, t}$ (cf. (4.8)) combined with the definition of $v(\cdot ; t, T)$ and the inequality established above, respectively. Consequently, equality holds which combined with the above yields

$$
v(\eta ; s, T)=\underset{\mathbb{Q} \in \mathcal{Q}_{s, t}}{\operatorname{ess} \inf } \underset{Z \in \mathcal{Z}_{t}^{a}}{\operatorname{ens}}\left\{\mathrm{E}^{\mathbb{Q}}\left[v\left(\eta \frac{Z_{s, t}}{Z_{s, t}^{\mathbb{Q}}} ; t, T\right) \mid \mathcal{F}_{s}\right]+\gamma_{s, t}(\mathbb{Q})\right\} .
$$

Hence, $v(\cdot ; t, T)$ is self-generating for $t \leq T$.

\footnotetext{
${ }^{13}$ Note that this argument highlights the need for the presence of $\kappa$.

${ }^{14}$ By relying on a result due to Neveu [100], the above inequality and, thus, the sub-martingal property, can be proven without the assumption that there exists an optimal strategy for which the infimum is attained.
} 
Proof of Lemma 4.14. First, we show that i) implies ii). To this end, assume that $V$ is selfgenerating. That is to say, for any $T>0$ and $t \leq T$, it holds that

$$
V(\eta, t)=\underset{\mathbb{Q} \in \mathcal{Q}_{t, T}}{\operatorname{ess} \inf } \operatorname{ess} \inf _{Z \in \mathcal{Z}_{T}^{a}}\left\{\mathrm{E}^{\mathbb{Q}}\left[V\left(\eta Z_{t, T} / Z_{t, T}^{\mathbb{Q}}, T\right) \mid \mathcal{F}_{t}\right]+\gamma_{t, T}(\mathbb{Q})\right\}, \quad \eta \in L_{+}^{0} .
$$

Let $y>0, \tilde{Z} \in \mathcal{Z}_{T}^{a}$ and $\tilde{\mathbb{Q}} \in \mathcal{Q}_{t, T}$, Let $\eta:=y \tilde{Z}_{t} / Z_{t}^{\tilde{\mathbb{Q}}}$. Then, it follows that

$$
\begin{aligned}
V\left(y \tilde{Z}_{t} / Z_{t}^{\tilde{\mathbb{Q}}}, t\right) & =\underset{\mathbb{Q} \in \mathcal{Q}_{t, T}}{\operatorname{ess} \inf \inf _{T}^{a}}\left\{\mathrm{E}^{\mathbb{Q}}\left[V\left(y \frac{\tilde{Z}_{t} Z_{t, T}}{Z_{t}^{\tilde{\mathbb{Q}}} Z_{t, T}^{\mathbb{Q}}}, T\right) \mid \mathcal{F}_{t}\right]+\gamma_{t, T}(\mathbb{Q})\right\} \\
& \leq \mathrm{E}^{\tilde{\mathbb{Q}}}\left[V\left(y \tilde{Z}_{T} / Z_{T}^{\tilde{\mathbb{Q}}}, T\right) \mid \mathcal{F}_{t}\right]+\gamma_{t, T}(\tilde{\mathbb{Q}}),
\end{aligned}
$$

which yields (4.11).

Next, as $V(y, t), t \in[0, \bar{T}]$, is self-generating by assumption, it is finite. Hence, due to assumption, there exists $\bar{Z} \in \mathcal{Z}_{\bar{T}}^{a}$ and $\overline{\mathbb{Q}} \in \mathcal{Q}_{0, \bar{T}}$ such that

$$
v(y ; 0, \bar{T})=\mathrm{E}^{\overline{\mathbb{Q}}}\left[V\left(y \bar{Z}_{\bar{T}} / Z_{\bar{T}}^{\overline{\mathbb{Q}}}, \bar{T}\right)\right]+\gamma_{0, \bar{T}}(\overline{\mathbb{Q}}) .
$$

We aim to show that for any $t \leq T \leq \bar{T},(4.11)$ holds with equality for the choice of $\mathbb{Q}_{T}:=\overline{\mathbb{Q}} \mid \mathcal{F}_{T}$ and $Z^{T}:=\mathrm{E}\left[\bar{Z} \mid \mathcal{F}_{T}\right]$. To this end, we first claim that $v(y ; 0, T)$ is attained by $\mathbb{Q}_{T}$ and $Z^{T}$ for all $T \in[0, \bar{T}]$. Indeed, use of (4.8) and (4.11) yields

$$
\begin{aligned}
v(y ; 0, \bar{T}) & =\mathrm{E}^{\overline{\mathbb{Q}}}\left[V\left(y \bar{Z}_{\bar{T}} / Z_{\bar{T}}^{\overline{\mathbb{Q}}}, \bar{T}\right)\right]+\gamma_{0, \bar{T}}(\overline{\mathbb{Q}}) \\
& =\mathrm{E}^{\overline{\mathbb{Q}}}\left[\mathrm{E}^{\overline{\mathbb{Q}}}\left[V\left(y \bar{Z}_{T} \bar{Z}_{T, \bar{T}} / Z_{T}^{\overline{\mathbb{Q}}} Z_{T, \bar{T}}^{\overline{\mathbb{Q}}}, \bar{T}\right) \mid \mathcal{F}_{T}\right]+\gamma_{T, \bar{T}}(\overline{\mathbb{Q}})\right]+\gamma_{0, T}(\overline{\mathbb{Q}}) \\
& \geq \mathrm{E}^{\overline{\mathbb{Q}}}\left[V\left(y \bar{Z}_{T} / Z_{T}^{\overline{\mathbb{Q}}}, T\right)\right]+\gamma_{0, T}(\overline{\mathbb{Q}}) \geq v(y ; 0, T),
\end{aligned}
$$

where the inequalities follow as $V(y, t)$ is self-generating and $\overline{\mathbb{Q}} \in \mathcal{Q}_{0, \bar{T}}$ implies that $\overline{\mathbb{Q}}_{T} \in \mathcal{Q}_{0, T}$ as well as $\overline{\mathbb{Q}} \in \mathcal{Q}_{T, \bar{T}}$. However, $v(y ; 0, T)=v(y ; 0, \bar{T})$ (once again due to self-genreration) and, thus, equality holds which completes the proof of the claim.

In order to show that (4.11) holds with equality for $\mathbb{Q}_{T}$ and $Z^{T}$ for all $T \in[0, \bar{T}]$, fix $t \leq T \leq \bar{T}$ and assume contrary to the claim that there exists $\varepsilon>0$ and $A \in \mathcal{F}_{t}, \mathbb{P}(A)>0$, such that

$$
V\left(y \bar{Z}_{t} / Z_{t}^{\overline{\mathbb{Q}}}, t\right)+\epsilon \mathbb{1}_{A} \leq \mathrm{E}^{\overline{\mathbb{Q}}}\left[V\left(y \bar{Z}_{T} / Z_{T}^{\overline{\mathbb{Q}}}, T\right) \mid \mathcal{F}_{t}\right]+\gamma_{t, T}(\overline{\mathbb{Q}}) .
$$

Taking the expectation under $\overline{\mathbb{Q}}$ we then obtain

$$
\mathrm{E}^{\overline{\mathbb{Q}}}\left[V\left(y \bar{Z}_{t} / Z_{t}^{\overline{\mathbb{Q}}}, t\right)\right]+\epsilon \overline{\mathbb{Q}}(A) \leq \mathrm{E}^{\overline{\mathbb{Q}}}\left[V\left(y \bar{Z}_{T} / Z_{T}^{\overline{\mathbb{Q}}}, T\right)\right]+\gamma_{0, T}(\overline{\mathbb{Q}})-\gamma_{0, t}(\overline{\mathbb{Q}}) .
$$

However, as $v(y ; 0, T)=v(y ; 0, \bar{T})$, this contradicts the above (cf. (4.57)). Hence, we conclude that (4.11) must hold with equality. 
In order to prove that ii) implies i), it suffices to show that for any $0<t<T<\infty$ and $\eta \in L_{+}^{0}\left(\mathcal{F}_{t}\right)$, it holds that

$$
V(\eta, t) \leq \mathrm{E}^{\mathbb{Q}}\left[V\left(\eta Z_{t, T} / Z_{t, T}^{\mathbb{Q}}, T\right) \mid \mathcal{F}_{t}\right]+\gamma_{t, T}(\mathbb{Q})
$$

for all $\mathbb{Q} \in \mathcal{Q}_{t, T}$ and $Z \in \mathcal{Z}_{T}^{a}$ and that there exists some $\hat{\mathbb{Q}} \in \mathcal{Q}_{t, T}$ and $\hat{Z} \in \mathcal{Z}_{T}^{a}$ for which equality holds. Note that (4.11) implies that for $\tilde{\eta}=\sum_{k=1}^{n} y_{k} \mathbb{1}_{A_{k}}$ a simple, positive and $\mathcal{F}_{t}$-measurable random variable,

$$
V\left(\tilde{\eta} Z_{t} / Z_{t}^{\mathbb{Q}}, t\right) \leq \mathrm{E}^{\mathbb{Q}}\left[V\left(\tilde{\eta} Z_{T} / Z_{T}^{\mathbb{Q}}, T\right) \mid \mathcal{F}_{t}\right]+\gamma_{t, T}(\mathbb{Q}),
$$

for all $\mathbb{Q} \in \mathcal{Q}_{t, T}$ and $Z \in \mathcal{Z}_{T}^{a}$. By use of a similar argument to the one used in the proof of Theorem 3.14 in [129], this can be verified to imply that (4.59) holds for arbitrary $\tilde{\eta} \in L_{+}^{0}\left(\mathcal{F}_{t}\right)$. For any $\mathbb{Q} \in \mathcal{Q}_{t, T}$ and $Z \in \mathcal{Z}_{T}^{a}$, (4.58) is then obtained by letting $\tilde{\eta}=\eta Z_{t}^{\mathbb{Q}} / Z_{t}$. Equality in (4.58) follows by a similar argument where all the inequalities are turned into equalities by the choice of $\mathbb{Q}_{T} \in \mathcal{Q}_{t, T}$ and $Z^{T} \in \mathcal{Z}_{T}^{a}$ for which (4.11) holds with equality.

Next, we show the equivalence between i) and iii). Given a sequence as specified in iii), ii) trivially holds. Hence, it only remains to show that i) implies iii).

First, we first claim that for each $y>0$, there exists $Z \in \mathcal{Z}^{a}$ and a sequence $\left(\mathbb{Q}_{T^{i}}\right), i=1, \ldots$, such that $\mathbb{Q}_{T^{i}}=\left.\mathbb{Q}_{T^{i+1}}\right|_{\mathcal{F}_{T^{i}}}$ and $\mathbb{Q}_{T}:=\left.\mathbb{Q}_{T^{i}}\right|_{\mathcal{F}_{T}} \in \mathcal{Q}_{0, T}$ for $T \leq T^{i}$. Moreover, for all $T>0$, (4.57) holds for $\bar{T}=T$ for the choice of $\mathbb{Q}_{T}$ and $Z_{T}$.

To this end, fix two times $T_{1}$ and $T_{2}$ and let $\mathbb{Q}_{1} \in \mathcal{Q}_{T_{1}}$ and $Z^{1} \in \mathcal{Z}_{T_{1}}^{a}$ be the optimal arguments at which $v\left(y ; 0, T_{1}\right)$ is attained. Then, in turn, let $\mathbb{Q}^{*} \in \mathcal{Q}_{T_{2}}$ and $Z^{*} \in \mathcal{Z}_{T_{2}}$ be the optimal arguments at which $v\left(y Z_{T_{1}}^{1} / Z_{T_{1}}^{\mathbb{Q}^{1}} ; T_{1}, T_{2}\right)$ is attained. Recall that existence of these arguments is ensured by assumption. Then, define $\mathbb{Q}_{2}$ and $Z^{2}$ via

$$
\frac{\mathrm{d} \mathbb{Q}_{2}}{\left.\mathrm{~d} \mathbb{P}\right|_{\mathcal{F}_{T_{2}}}}=Z_{T_{1}}^{\mathbb{Q}_{1}} Z_{T_{1}, T_{2}}^{\mathbb{Q}^{*}} \quad \text { and } \quad Z^{2}=Z_{T_{1}}^{1} Z_{T_{1}, T_{2}}^{*}
$$

Use of the fact that $V(y, t)$ is self-generating then yields

$$
\begin{aligned}
v\left(y ; 0, T_{1}\right) & =\mathrm{E}\left[Z_{T_{1}}^{\mathbb{Q}^{1}} V\left(y Z_{T_{1}}^{1} / Z_{T_{1}}^{\mathbb{Q}^{1}}, T_{1}\right)\right]+\gamma_{0, T_{1}}\left(\mathbb{Q}^{1}\right) \\
& =\mathrm{E}\left[Z_{T_{1}}^{\mathbb{Q}^{1}}\left(\mathrm{E}\left[Z_{T_{1}, T_{2}}^{\mathbb{Q}^{*}} V\left(y \frac{Z_{T_{1}}^{1} Z_{T_{1}, T_{2}}^{*}}{Z_{T_{1}}^{\mathbb{Q}_{1}^{1}} Z_{T_{1}, T_{2}}^{\mathbb{Q}^{*}}}, T_{2}\right) \mid \mathcal{F}_{T_{1}}\right]+\gamma_{T_{1}, T_{2}}\left(\mathbb{Q}^{*}\right)\right)\right]+\gamma_{0, T_{1}}\left(\mathbb{Q}^{1}\right) \\
& =\mathrm{E}^{\mathbb{Q}^{2}}\left[V\left(y Z_{T_{2}}^{2} / Z_{T_{2}}^{\mathbb{Q}^{2}}, T_{2}\right)\right]+\gamma_{0, T_{2}}\left(\mathbb{Q}^{2}\right) \geq v\left(y ; 0, T_{2}\right)
\end{aligned}
$$

where it under assumption b) was used that $V^{-}\left(\zeta, T_{2}\right) \in L^{1}\left(\mathcal{F}_{T_{2}} ; \mathbb{Q}^{2}\right)$, for all $\zeta \in L^{0}\left(\mathcal{F}_{T_{2}}\right)$, which (using that $v\left(y ; 0, T_{1}\right)$ is finite) implies that $\mathrm{E}\left[Z^{\mathbb{Q}_{1}} \gamma_{T_{1}, T_{2}}\left(\mathbb{Q}^{*}\right)\right]<\infty$ and, thus, that $\mathbb{Q}_{2} \in \mathcal{Q}_{0, T_{2}}$. Under assumption a), the fact that $\mathbb{Q}_{2} \in \mathcal{Q}_{0, T_{2}}$ follows directly by the fact that $\mathcal{Q}_{t, T}$ is stable under pasting. Moreover, $Z^{2} \in \mathcal{Z}_{T_{2}}^{a}$. As $V(y, t)$ is self-generating by assumption, 
$v\left(y ; 0, T_{1}\right)=v\left(y ; 0, T_{2}\right)$ and, thus, equality holds in (4.60). Consequently, $v\left(y ; 0, T_{2}\right)$ is attained for the choice of $\mathbb{Q}^{2}$ and $Z^{2}$.

Use of the same argument as in the proof of i) implies ii), then yields that (4.57) holds for $\bar{T}=T$ for the choice of $\mathbb{Q}_{T}$ and $Z^{T}$, for any $T \leq T_{2}$. Repetition of the above procedure, then gives a sequence satisfying the claim. That the sequence $\mathbb{Q}_{T_{i}}, i=1, \ldots$, and $Z \in \mathcal{Z}^{a}$ satisfying the claim also satisfies condition iii), follows by use of the same argument as used in the proof of i) implies ii). 


\section{Chapter 5}

\section{Ambiguity averse portfolio optimization with respect to quasiconcave utility functionals}

In this chapter we study risk- and ambiguity-averse investment criteria defined in terms of quasiconcave utility functionals. Terminal payoffs are, thus, evaluated according to robust utility functionals of the form

$$
X \longmapsto \inf _{\mathbb{Q} \ll \mathbb{P}} G\left(\mathbb{Q}, \mathrm{E}^{\mathbb{Q}}[U(X)]\right),
$$

where $G$ is the dual function in the robust representation (to be specified below) of some quasiconcave utility functional. We refer to the Introduction in Chapter 1 for the motivation and an overview of the related literature; see pp. 6-11.

The chapter is organized as follows. In Section 5.1, the market model and the investment criterion are specified. The main results are presented in Section 5.2 while the proofs and further remarks are given in Section 5.3.

\subsection{The market model and the investment criterion}

We consider a fixed finite horizon $T>0$ and a given filtered probability space $\left(\Omega, \mathbb{F},\left(\mathcal{F}_{t}\right)_{t \in[0, T]}, \mathbb{P}\right)$, where $\mathbb{P}$ is the so-called reference measure. We first precise the investor's preferences. Then, in turn, the market model and the investment problem are specified.

The investor's risk preferences are quantified via a utility function $U:(0, \infty) \rightarrow \mathbb{R}$, which is strictly increasing, strictly concave and satisfies the Inada conditions,

$$
\lim _{x \rightarrow 0} U^{\prime}(x)=\infty \quad \text { and } \quad \lim _{x \rightarrow \infty} U^{\prime}(x)=0 .
$$


As argued in the Chapter 1, following [25], the investor's ambiguity aversion is specified via a quasiconcave utility functional. The latter is a mapping $\phi: L^{\infty} \rightarrow[-\infty, \infty]$ that satisfies quasiconcavity and monotonicity. Recall that a function is quasiconcave if it is the negative of a quasiconvex function and that a function is quasiconvex if its level-sets are convex. For a function $f: \mathcal{X} \rightarrow \mathbb{R}$, that is equivalent to $f(\lambda x+(1-\lambda) y) \leq \max \{f(x), f(y)\}, x, y \in \mathcal{X}$. Hence, a quasiconcave utility functional (the negative of a quasiconvex risk measure) satisfies

$$
\phi(\lambda X+(1-\lambda) Y)) \geq \min \{\phi(X), \phi(Y)\}, \quad \text { and } \quad \phi(X) \geq \phi(Y), \text { if } X \geq Y, \mathbb{P} \text {-a.s. }
$$

However, it need satisfy neither cash-invariance nor positive homogeneity.

The specification of our criterion relies on the robust representation result (Theorem 3.2 in [35]; see also Theorem 7 in [25]) stating that every $\sigma\left(L^{\infty}, L^{1}\right)$-upper semicontinuous quasiconcave utility functional $\phi(X)$ admits the (robust) representation

$$
\phi(X)=\inf _{\mathbb{Q} \in \mathcal{M}_{1}(\mathbb{P})} G\left(\mathbb{Q}, \mathrm{E}^{\mathbb{Q}}[X]\right)
$$

for some function $G \in \mathcal{G}$, where the set of functions $\mathcal{G}$ is specified next and $\mathcal{M}_{1}(\mathbb{P})$ is the set of $\sigma$-additive probability measures absolutely continuous with respect to $\mathbb{P}$. Conversely, for any $G \in \mathcal{G}$, the function $\phi$ defined in this way is a upper semicontinuous quasiconcave utility functional.

Definition 5.1. Let $\mathcal{G}$ denote the set of functions $G: \mathcal{M}_{1}(\mathbb{P}) \times \mathbb{R} \rightarrow[-\infty, \infty]$ satisfying the following conditions:

i) $G(\mathbb{Q}, \cdot)$ is non-decreasing and right-continuous;

ii) $G$ is jointly quasiconvex;

iii) $G^{-}(\cdot, s)$ is weakly lower semicontinuous, where

$$
G^{-}(\mathbb{Q}, s)=\sup _{t<s} G(\mathbb{Q}, t), \quad \mathbb{Q} \in \mathcal{M}_{1}(\mathbb{P})
$$

iv) $G$ has an asymptotic maximum in the sense that

$$
A M(G):=\lim _{s \rightarrow \infty} G(\mathbb{Q}, s)=\lim _{s \rightarrow \infty} G(\overline{\mathbb{Q}}, s), \quad \text { for all } \mathbb{Q}, \overline{\mathbb{Q}} \in \mathcal{M}_{1}(\mathbb{P})
$$

In particular, $G(\mathbb{Q}, \cdot)$ is quasi-linear and upper semicontinuous.

Combined, the above implies that we consider an investor assessing the utility of terminal payoffs, modelled as random variables on $(\Omega, \mathcal{F}, \mathbb{P})$, in terms of a robust utility functional of the form (5.1) with $G \in \mathcal{G}$. For the specific cases when $G$ corresponds to a coherent and concave utility functional, the criterion reduces, respectively, to the multiple priors and variational preferences 
studied in $[106,111]$ and [110]. In the same way as the study of the latter problems were motivated by the axiomatic results in [54] and [87], the study of the more general quasiconcave case relies on the recent axiomatic extensions in [25].

Without loss of generality, we may precise (5.1) by writing

$$
X \rightarrow \inf _{\mathbb{Q} \in \mathcal{Q}} G\left(\mathbb{Q}, \mathrm{E}^{\mathbb{Q}}[U(X)]\right)
$$

where, for given $G \in \mathcal{G}$,

$$
\mathcal{Q}:=\left\{\mathbb{Q} \in \mathcal{M}_{1}(\mathbb{P}): G(\mathbb{Q}, t)<\infty, \text { for some } t>0\right\} .
$$

We stress that while $U(X) \in L^{0}$ for the type of payoffs we are to consider (cf. below), the utility functionals defined with respect to $G \in \mathcal{G}$ are defined only for bounded random variables. Indeed, while the theory of quasiconcave risk measures has been extended to more general spaces, we do not restrict to functions $G \in \mathcal{G}$ for which that holds. In doing so, we follow [110] who in a similar manner studied the risk- and ambiguity-averse investment problem formulated with respect to penalty functions associated with convex risk measures on $L^{\infty}$. In order to ensure that (5.3) is well defined, we let $\mathrm{E}^{\mathbb{Q}}[F]:=\infty$ if $\mathrm{E}^{\mathbb{Q}}\left[F^{+}\right]=\mathrm{E}^{\mathbb{Q}}\left[F^{-}\right]=\infty$. Moreover, we extend the domain of $G(\mathbb{Q}, \cdot)$ to the extended real line, in that we define $G(\mathbb{Q},-\infty):=-\infty$ and $G(\mathbb{Q}, \infty):=A M(G)$.

Example 5.2. An example of preferences defined in terms of a quasiconcave utility functional are the so-called smooth criteria:

$$
X \longmapsto \phi^{(-1)}\left(\int_{\mathcal{M}_{1}(\mathbb{P})} \phi\left(\mathrm{E}^{\mathbb{Q}}[U(X)]\right) d \mu(\mathbb{Q})\right),
$$

where $\phi$ is an increasing and concave function modelling the ambiguity aversion. That is, rather than taking the infimum over possible models, the agent considers a distribution $\mu$ over them. Such criteria were axiomatized in Klibanoff et al. [77] (see also [116]) under axioms stronger than those used in [25]. Hence, (for bounded payoffs) these criteria constitute a particular case of the quasiconcave preferences. The specific form of the associated function $G$ is given in [25] and yields further intuition for the criteria. In particular, this motivates the question of whether these results hold also for general terminal payoffs. We belive this to be the case but leave for future research to thoroughly investigate this.

Next, we specify the continuous-time market model and the set of admissible trading strategies. The market model is defined by a $\mathbb{R}^{d}$-valued price process $S_{t}$, which is assumed to be a semimartingale on $\left(\Omega, \mathbb{F},\left(\mathcal{F}_{t}\right), \mathbb{P}\right)$. The price-process $S_{t}$ is assumed to satisfy the condition of NFLVR with respect to the reference-measure $\mathbb{P}$. For given initial capital $x>0$, and a predictable $S$-integrable trading strategy $\pi_{t}$, the associated wealth-process is given by

$$
X_{t}^{\pi}=x+\int_{0}^{t} \pi_{s} d S_{s}, \quad t \geq 0
$$


We denote a trading strategy admissible if $X_{t}^{\pi} \geq 0, \mathbb{P}$-a.s. for $t \in[0, T]$ and denote the associated set of wealth-processes by $\mathcal{X}(x)$.

In consequence, we aim at studying the following investment problem.

Problem 5.3. For given $G \in \mathcal{G}$, we consider the risk- and ambiguity-averse investment problem of maximizing the functional (5.3) over admissible terminal payoffs $X_{T}^{\pi}, X \in \mathcal{X}(x)$. The associated value-function $u: \mathbb{R}_{+} \rightarrow \mathbb{R}$ is defined by

$$
u(x):=\sup _{X \in \mathcal{X}(x)} \inf _{\mathbb{Q} \in \mathcal{Q}} G\left(\mathbb{Q}, \mathrm{E}^{\mathbb{Q}}\left[U\left(X_{T}^{\pi}\right)\right]\right) .
$$

We stress that the set of admissible strategies $\mathcal{X}$ is defined with respect to $\mathbb{P}$, the role of which is to specify the null-sets rather than representing the most likely model. In particular, we consider a dominated setting in which all measures $\mathbb{Q} \in \mathcal{Q}$ are absolutely continuous with respect to the reference measure (for ambiguity averse portfolio optimization with mutually singular measures, see [101] and the references therein). Also note that while $u(x)$ is non-decreasing, it need be neither concave nor continuous (cf. Remark 5.11).

We conclude this section by defining an auxiliary optimization problem which will be crucial for the analysis that follows. For $\mathbb{Q} \ll \mathbb{P}$, let

$$
u_{\mathbb{Q}}(x):=\sup _{X \in \mathcal{X}(x)} \mathrm{E}^{\mathbb{Q}}\left[U\left(X_{T}\right)\right]
$$

where $\mathcal{X}(x)$ is as defined above. We also introduce the (dual) auxiliary problem

$$
v_{\mathbb{Q}}(y):=\inf _{Y \in \mathcal{Y}(y)} \mathrm{E}\left[Z V\left(Y_{T} / Z\right)\right]
$$

where $Z=\frac{\mathrm{d} \mathbb{Q}}{\mathrm{d} \mathbb{P}}, V(y)=\sup _{x \geq 0}(U(x)-x y)$ and $\mathcal{Y}(y)$ is the set of all positive $\mathbb{P}$-supermartingales such that $Y_{0}=y$ and $X Y$ is a $\mathbb{P}$-supermartingale for all $X \in \mathcal{X}(1)$. Without further notice, these functions will also be denoted by $u_{Z}(x)$ and $u_{Z}(y)$, respectively. While the objective functions in (5.6) and (5.7) are defined with respect to the measure $\mathbb{Q}$, the set of admissible strategies and dual objects are defined with respect to the reference measure $\mathbb{P}$. Hence, while it holds for $\mathbb{Q} \sim \mathbb{P}$, that these auxiliary problems are, respectively, the standard investment problem and its dual counterpart for the market defined with respect to the measure $\mathbb{Q}$, this need not be the case for $\mathbb{Q} \ll \mathbb{P}$. In particular, $S_{t}$ need not satisfy the condition of NFLVR with respect to $\mathbb{Q}$ (see further discussion in Section 5.3.1 and Remarks 5.14 and 5.15 below). 


\subsection{The main results}

In this section, we present the main results. The proofs are given in Section 5.3. The following standing assumption is imposed throughout.

Assumption 5.4. There exists $\mathbb{Q}_{0}, \mathbb{Q}_{1} \in \mathcal{Q}$ such that

$$
u_{\mathbb{Q}_{0}}\left(x_{0}\right)<\infty, \text { for some } x_{0}>0
$$

and

$$
G\left(\mathbb{Q}_{1}, u_{\mathbb{Q}_{1}}(x)\right)<\infty, \text { for all } x>0 .
$$

Note that assumptions (5.8) and (5.9) are disjoint in the sense that none of them implies the other (although, they might both be satisfied for the same $\mathbb{Q} \in \mathcal{Q}$ ). Also note that (5.8) implies $u_{\mathbb{Q}_{0}}(x)<\infty$ for all $x>0$ and, furthermore, that Assumption 5.4 yields

$$
u(x)=\sup _{g \in \mathcal{X}(x)} \inf _{\mathbb{Q} \in \mathcal{Q}} G\left(\mathbb{Q}, \mathrm{E}^{\mathbb{Q}}[U(f)]\right) \leq \inf _{\mathbb{Q} \in \mathcal{Q}} G\left(\mathbb{Q}, u_{\mathbb{Q}}(x)\right)<\infty, \quad x>0 .
$$

First we establish existence of a solution to Problem 5.3. The question of uniqueness is discussed below.

Theorem 5.5. Let $G \in \mathcal{G}$ such that Assumption 5.4 holds and assume either that

$$
v_{\mathbb{Q}}(y)<\infty, \quad y>0
$$

for each $\mathbb{Q} \in \mathcal{Q}$, or that $G(\cdot, t), t \in \mathbb{R}$, is convex and (5.11) holds for each $\mathbb{Q} \in \mathcal{Q}_{e}$. Then, there exists an optimal terminal payoff $\hat{X} \in \mathcal{X}(x)$ for which the supremum in (5.5) is attained.

We make two general remarks on the assumptions of the above result.

Remark 5.6. That $G(\cdot, t), t \in \mathbb{R}$, is convex is a necessary but not a sufficient condition for the associated utility functional to be concave. This class of preferences therefore includes but is not limited to the variational ones. Nevertheless, the very same methods as introduced in [110] can be used to establish existence for this case, cf. the discussion in Section 5.3.1, and in consequence the weaker condition that (5.11) only holds for $\mathbb{Q} \in \mathcal{Q}_{\text {e }}$ suffices.

Remark 5.7. The assumption that (5.11) holds for $\mathbb{Q} \in \mathcal{Q}$ implies (cf. Lemma 5.12 below) that $\mathcal{Q}=\mathcal{Q}^{f}$, where

$$
\mathcal{Q}^{f}:=\left\{\mathbb{Q} \in \mathcal{Q}: u_{\mathbb{Q}}(x)<\infty\right\}
$$


In fact, a sufficient condition for Theorem 5.5 to hold, is that $\mathcal{Q}=\mathcal{Q}^{f}$ and $A E_{+\infty}(U)<1$. Indeed, similarly to the standard case (cf. Note 2 in [80]), this yields the finiteness of $v_{\mathbb{Q}}(y)$, $\mathbb{Q} \in \mathcal{Q}$. Yet another sufficient condition is that $v_{\mathbb{Q}}(y)<\infty, \mathbb{Q} \in \mathcal{Q}_{e}$, and that for each $\mathbb{Q}_{0} \in \mathcal{Q}$, there exists $\mathbb{Q}_{1} \in \mathcal{Q}_{e}$ such that

$$
\mathrm{E}^{\mathbb{Q}_{0}}[U(g)] \leq \mathrm{E}^{\mathbb{Q}_{1}}[U(g)]<\infty, \text { for all } g \in \mathcal{C}(x) .
$$

Indeed, (5.12) implies that $u_{\mathbb{Q}_{0}}(y) \leq u_{\mathbb{Q}_{1}}(y)<\infty$, where the last inequality follows from the assumption. Hence, the conjugacy relations of Lemma 5.12 below holds with respect to $\mathbb{Q}_{0}$. This yields,

$$
v_{\mathbb{Q}_{0}}(y)=\sup _{x>0}\left(u_{\mathbb{Q}_{0}}(y)-x y\right) \leq \sup _{x>0}\left(u_{\mathbb{Q}_{1}}(y)-x y\right)=v_{\mathbb{Q}_{1}}(y)<\infty .
$$

In consequence, $v_{\mathbb{Q}_{0}}(y)<\infty$ for all $\mathbb{Q}_{0} \in \mathcal{Q}$ and the assumptions of Theorem 5.5 hold.

We stress that the assumption that $v_{\mathbb{Q}}(y)<\infty, \mathbb{Q} \in \mathcal{Q}$, is rather natural. First, under some additional assumptions, it is established below (cf. Theorem 5.9) that the infimum and supremum in (5.5) can be interchanged. In effect, the set $\mathcal{Q}$ can be replaced by the set $\mathcal{Q}^{f}$ without affecting the indirect utility $u(x)$. More importantly, this assumption implies that the auxiliary investment problem, itself, is solvable for each individual measure $\mathbb{Q} \in \mathcal{Q}$ (cf. Lemma 5.12 below). As pointed out above, the auxiliary problem is not a standard one. Nevertheless this might be understood as an arbitrage condition put on each individual model (cf. [71] where it is shown that the classical utility maximization problem admits a solution if and only if the market satisfies the no arbitrage condition $\mathrm{NA}_{1}$ ). The assumption therefore relates to the interpretation of the ambiguity averse criterion. On the one hand, the criterion (5.3) emerges due to the axioms posed on the preferences; this via the robust representation of quasiconcave utility functionals. This motivation - per se - does not imply that the measures $\mathbb{Q} \in \mathcal{Q}$ satisfy any market related conditions. However, it is common in the literature to also motivate ambiguity averse criteria by the fact that they, effectively, amount to taking the expectation with respect to different possible market models $\mathbb{Q}$ weighted according to their plausibility. While the weighting is determined by the penalty function $\gamma$ for the variational case, the function $G$ allows for more flexibility in the quasiconcave case. Given the latter interpretation, it is natural to assume that each market model $\mathbb{Q} \in \mathcal{Q}$, itself constitutes a sensible market model which excludes arbitrage. This is exactly what (5.11) guarantees. The important question of how to ensure that this condition holds for specific choices of $G$ remains to be addressed.

Under the assumptions of Theorem 5.5, it is not clear whether the infimum and supremum in (5.5) can be interchanged (neither whether the infimum is attained). Hence, there need not exist an auxiliary problem (cf. (5.6)) producing the same investment behaviour as the ambiguity averse criterion (5.3). This is, however, the case under some additional assumptions; we will come back to this (see page 136). 
Next, we turn to the study of the dual version of the risk and ambiguity averse investment problem (5.3). Specifically, we establish relations between the primal and dual problems and their respective solutions. As holds for the variational case (cf. [110]), the study of the dual problem is, in fact, not needed for proving existence of a solution to the primal problem. Indeed, the proof of Theorem 5.5 relies on properties of the auxiliary problem (5.6) and, thus, rather on the study of the dual version thereof. Nevertheless, the study of its dual counterpart is of interest as it gives a further understanding of the problem and of the optimal strategy. Similarly to the standard case, the study of the dual problem rather than the primal one offers various advantages. This is particularly evident for ambiguity averse preferences where the dual problem amounts to the search for a pure infimum, whereas the primal problem features a saddle-point. In particular, most articles providing explicit solutions for specific choices of utility and penalty functions, notably focus on the dual rather than the primal problem. Hence, there is reason to believe that the dual formulation is helpful in obtaining more explicit results also for the quasiconcave case. The results also enable us to draw important conclusions about the optimal strategy. Specifically, regarding the existence of an equivalent auxiliary problem and uniqueness of the optimal strategy (cf. the discussion after Theorem 5.10).

We impose the following additional assumption.

Assumption 5.8. The function $G$ is jointly lower semicontinuous and the level sets $\mathcal{Q}_{t}(c)$ are relatively weakly compact, where

$$
\mathcal{Q}_{t}(c):=\{\mathbb{Q} \in \mathcal{Q}: G(\mathbb{Q}, t) \leq c\}, \quad t \in \mathbb{R}, c \geq 0 .
$$

Furthermore, either $U: \mathbb{R}_{+} \rightarrow \mathbb{R}_{+}$or $G(\mathbb{Q}, \cdot), \mathbb{Q} \in \mathcal{Q}$, is concave.

Note that since $\mathbb{Q} \rightarrow G(\mathbb{Q}, t)$ is weakly lower semicontinuous, $\mathcal{Q}_{t}(c)$ is weakly closed and, thus, due to Dunford-Pettis theorem, relative weak compactness is equivalent to uniform integrability. Moreover, recall that for functions $G \in \mathcal{G}, G^{-}(\cdot, t), t \in \mathbb{R}$, is lower semicontinuous and $G(\mathbb{Q}, \cdot)$, $\mathbb{Q} \in \mathcal{Q}$, is upper semicontinuous. Hence, the assumption that $G$ is jointly lower semicontinuous is, in fact, equivalent to the assumption that $G(\mathbb{Q}, \cdot)$ is continuous. Both these properties are closely related to the fact that one considers (evenly) quasiconcave utility functionals which are continuous not only from above but also from below and, thus, weakly continuous (as opposed to weakly upper semicontinuous). We refer to $[24,45,49,50]$ for further details but note that this class of quasiconcave utility functionals is natural within the present context. Indeed, in [25] the axioms were formulated (including a continuity axiom) so as to render a numerical representation in terms of such weakly continuous utility functionals.

While this (natural) additional continuity assumption is crucial for the duality results, the restriction to positive utility functions is needed for technical reasons. The possibility of relaxing 
this assumption is left for future study. Note, however, that the results in, among others, [106] are established only for positive utility functions. The restriction to $G(\mathbb{Q}, \cdot)$ concave yields, essentially, the variational case treated in [110]. It is included as a specific case in order to compare our methods and relate our results to the ones therein.

Theorem 5.9. Let $G \in \mathcal{G}$ such that Assumptions 5.4 and 5.8 hold. Then,

i) the robust value-function satisfies

$$
u(x):=\sup _{X \in \mathcal{X}(x)} \inf _{\mathbb{Q} \in \mathcal{Q}} G\left(\mathbb{Q}, \mathrm{E}^{\mathbb{Q}}\left[U\left(X_{T}\right)\right]\right)=\inf _{\mathbb{Q} \in \mathcal{Q}} \sup _{X \in \mathcal{X}(x)} G\left(\mathbb{Q}, \mathrm{E}^{\mathbb{Q}}\left[U\left(X_{T}\right)\right]\right),
$$

and, furthermore,

$$
u(x)=\inf _{y>0} v(y ; x),
$$

where $v(y ; x)$ is the dual value function given by

$$
v(y ; x):=\inf _{\mathbb{Q} \in \mathcal{Q}} G\left(\mathbb{Q}, v_{\mathbb{Q}}(y)+x y\right) .
$$

ii) Moreover, if $v(y ; x)<\infty$, then the dual problem $(5.15)$ admits a solution $(\widehat{\mathbb{Q}}, \widehat{Y})$ that is maximal in the sense that any other solution $(\mathbb{Q}, Y)$ satisfies $\mathbb{Q} \ll \widehat{\mathbb{Q}}$ and $Y_{T} / Z=\widehat{Y}_{T} / \widehat{Z}$, Q-a.s.

While the above result imposes stronger conditions on the structural properties of $G$ (and $U$ ) than needed for Theorem 5.5, finiteness assumptions of the type needed for the existence are not required here. This should be related to the results in $[79,80]$, where the conjugacy relations are proven under much weaker conditions than needed for the existence.

For the variational case, the relation between the primal and dual value function (cf. (5.14)) was established in [110] under the additional assumptions that $u_{\mathbb{Q}_{0}}(x)<\infty$ for some $\mathbb{Q}_{0} \in \mathcal{Q}_{e}$ and

$$
v(y ; x)<\infty \text { implies } v_{\mathbb{Q}_{1}}(y)<\infty \text { for some } \mathbb{Q}_{1} \in \mathcal{Q}_{e} \text {. }
$$

Although very natural, this assumption is, in fact, not needed. Indeed, the approach therein is based on replacing the set $\mathcal{Q}$ in $(5.13)$ by $\mathcal{Q}_{e}$ and, then, in turn, make use of duality relations for auxiliary problems defined with respect to measures which are equivalent to $\mathbb{P}$. This additional assumption $^{1}$ is needed to ensure that this can be done. As we have proven in Lemma 5.12 that the duality relations between $u_{\mathbb{Q}}(x)$ and $v_{\mathbb{Q}}(y)$ hold also for $\mathbb{Q} \ll \mathbb{P}$, it follows that this assumption, in fact, is not needed. We stress that it is very natural though. Moreover, for the

\footnotetext{
${ }^{1}$ Note that for the existence result, the strong finiteness-assumptions imposed on each individual model $\mathbb{Q} \in \mathcal{Q}_{e}$ in order to ensure existence, already implies that such limiting-procedures work for the variational case. Hence, our approach does not enable any weakening of the assumptions for the existence result.
} 
variational case, a slightly stronger result can be proven under this additional assumption (cf. Remark 5.15).

The next result relates the respective solutions to the primal and dual problems. In particular, the result yields the existence of an equivalent auxiliary problem and results on the uniqueness of the optimal strategy.

Theorem 5.10. Let $G \in \mathcal{G}$ and assume Assumption 5.8 and the assumptions of Theorem 5.5 to hold. Let $\bar{X}$ be a solution to the primal problem. Then, the primal problem admits a saddle point $(\bar{X}, \overline{\mathbb{Q}})$ and there exists $y^{*}>0$ for which the infimum in (5.14) is attained.

Assume, furthermore, that $G(\mathbb{Q}, \cdot), \mathbb{Q} \in \mathcal{Q}$, is strictly increasing and let $(\widehat{Y}, \widehat{\mathbb{Q}})$ be any solution to the dual problem at level $y^{*}$. Then, $(\bar{X}, \widehat{\mathbb{Q}})$ is a saddle point for the primal problem and, moreover,

$$
\bar{X}=I\left(\widehat{Y}_{T} / \widehat{Z}\right), \quad \widehat{\mathbb{Q}} \text {-a.s. }
$$

The above result is of particular interest as it gives a further understanding of the optimal strategy. Let $\widehat{\mathbb{Q}}$ be the measure in the maximal solution to the dual (or, equivalently, primal) problem. Following the related literature, we refer to it as the least favourable measure. Relation (5.17) then implies that the optimal solution $\widehat{X}_{T}$, in fact, is $\widehat{\mathbb{Q}}$-a.s. unique. Consequently, if the least favourable measure is equivalent to $\mathbb{P}$, the solution is $\mathbb{P}$-a.s. unique. In particular, it can then be recovered from the dual solution. In general, the least favourable measure need however not be equivalent to $\mathbb{P}$. Nevertheless, an optimal strategy can still be constructed from a given solution of the dual problem by superhedging of an appropriate claim (cf. Corollary 2.7 in [110]).

The existence of a saddle point also implies that, a posteriori, there exists an auxiliary investment problem (5.6) producing the same optimal behaviour as the original criterion (in particular, this always holds for the variational criteria). Specifically, the auxiliary problem defined with respect to the least favourable measure $\widehat{\mathbb{Q}}$ admits a solution (cf. Lemma 5.12 below) which $\widehat{\mathbb{Q}}$-a.s. coincides with the solution to the original problem. Since the least-favourable measure is part of the solution to the original problem, the equivalence between the ambiguity averse and the auxiliary problem is an a posteriori result. Given the existence of an auxiliary problem (for which the objective function is concave), the $\widehat{\mathbb{Q}}$-a.s. uniqueness of the optimal strategy is natural. Under the weaker assumptions of Theorem 5.5, it is not clear though whether an equivalent auxiliary problem exists (cf. the discussion on page 133). The question of to which extent uniqueness holds under more general assumptions (given $G(\mathbb{Q}, \cdot)$ strictly increasing) is left for future study. 
Remark 5.11 (Time consistency). In general, Problem 5.3 is not a time-consistent investment problem (see [110] for counter-examples in the variational case). A natural question is therefore under what assumptions it is. Indeed, time-consistency is essential for it is fundamentally linked to the definition and conceptual understanding of optimality. Moreover, it enables the use of stochastic control methods. In consequence, it is a prerequisite for extending to the quasiconcave case the explicit results obtained in terms of PDEs and BSDEs for the variational preferences (cf. the references in the Chapter 1). Time consistency of quasiconcave utility functionals (quasiconvex risk measures) remains, however, an open problem and, in particular, feasible explicit examples are few. Indeed, while necessary and sufficient conditions for temporal consistency of convex risk measures were established in [33] (see also [12] and [43]), such results are, yet, lacking for the quasiconvex case. In recent work, [49, 50] initiated such a programme via the study of conditional quasiconvex risk measures. Questions of temporal consistency and the relation to g-expectations have also been studied within the more general framework of non-linear expectations in [103]. In particular, it would be interesting to find an example of a quasiconcave utility functional defined in terms of a given BSDE and consider the associated investment problem.

We also note that in (5.5), the risk preferences of the investor are modelled via a standard continuous and concave utility function. While this assumption makes perfect sense for the static problem, the value function $u(x)$ will only satisfy weaker properties (a more precise study of the properties of the value function is left for future study). Hence, a study of time-consistent problems of this type also requires a study of the risk and ambiguity averse investment problem (5.5) under weaker assumptions on $U(x)$. In summary, while questions of time-consistency are of great interest, they impose challenging additional problems which are left for future study.

\subsection{Proofs}

The above theorems extend results established for the variational preferences in [110] (cf. [106, 111] for the coherent case) to the quasiconcave risk- and ambiguity-averse investment criteria. Naturally, our proofs are therefore inspired by and in many ways similar to those in the former articles. Specifically, also here the idea is to establish results for the risk- and ambiguity-averse problem by relying on existing results for the auxiliary problem (5.6). As pointed out above, for $\mathbb{Q} \sim \mathbb{P}$, this is the classical utility maximization problem as studied in $[79,80]$. For $\mathbb{Q} \ll \mathbb{P}$, this need not be the case. In [110], this is dealt with by use of certain limiting arguments. However, for our case, the weaker properties of $G$ imply that this approach does not apply. Hence the need for somewhat different arguments. 
In Section 5.3.1, we explain why we were not able to directly apply the arguments developed in [110] and describe the approach we use in further detail. This illustrates the differences in the required assumptions. The existence and duality results are proven, respectively, in Sections 5.3.2 and 5.3.3. The proof of Lemma 5.12 below is deferred to the Appendix.

\subsubsection{The auxiliary problem and its significance}

For the variational case studied in [110], the appearance of measures in $\mathcal{Q}$ which are absolutely continuous but not necessarily equivalent to $\mathbb{P}$, is addressed in the following way. For $\mathbb{Q}_{0} \in \mathcal{Q} \backslash \mathcal{Q}_{e}$, the authors let $\mathbb{Q}_{1} \in \mathcal{Q}_{e}$ and define the measures $\mathbb{Q}_{t}, t \in[0,1]$, via the Radon-Nikodym derivative $Z_{t}:=(1-t) Z_{0}+t Z_{1}$, where $Z_{0}$ and $Z_{1}$ correspond, respectively, to $\mathbb{Q}_{0}$ and $\mathbb{Q}_{1}$. Then, $\mathbb{Q}_{t} \in \mathcal{Q}_{e}$ for $t \in(0,1]$. Moreover, under suitable finiteness-assumptions, the functions

$$
t \rightarrow G\left(\mathbb{Q}_{t}, u_{\mathbb{Q}_{t}}(x)\right) \text { and } t \rightarrow G\left(\mathbb{Q}_{t}, v_{\mathbb{Q}_{t}}(y)+x y\right),
$$

are continuous and upper semicontinuous, respectively. Combined, this implies that the results can be established by relying on results for auxiliary problems (cf. (5.6)) defined with respect to measures equivalent to $\mathbb{P}$ only. To the latter, the results in $[79,80]$ can, in turn, be directly applied. In consequence, certain assumptions need only be posed on the set of measures $\mathcal{Q}_{e}$ as opposed to $\mathcal{Q}$ (cf. Theorem 5.5). This approach is closely related to the fact that the set of absolutely continuous measures in the representation of a concave utility functional, under the assumption that there exists an equivalent measure for which the penalty is finite, can be replaced by the equivalent ones; cf. [78].

The continuity properties in (5.18) rely, however, on the fact that for the variational case $G(\cdot, t)=$ $\gamma(\cdot)+t$ and, thus, the mapping is convex. Indeed, this implies that the mapping

$$
t \rightarrow G\left(Z_{t}, s\right), \quad t \in[0,1], s \in \mathbb{R}
$$

is convex as well. If it is finite, it is therefore upper-semicontinuous. According to Lemma 3.3 in [111], $t \rightarrow u_{Z_{t}}(x)$ is continuous and, under rather weak assumptions, also $t \rightarrow v_{Z_{t}}(y)$ is upper semicontinuous. Hence, (under suitable finiteness assumptions) the required continuity properties in (5.18) follow. The crucial point is that for the quasiconcave case we consider, the function $G(\cdot, t)$ might not be convex. Therefore, the continuity properties in (5.18) might not hold and, in consequence, the arguments developed in [110] do not apply.

Our methods are rather based on a closer study of the auxiliary problem (5.6). Although not a standard problem, it is, in fact, only a modification thereof and can therefore be solved by use of the same methods as developed in [79] (cf. Lemma 5.12 below). By use of this observation, we then address the risk and ambiguity averse investment problem. In particular, we obtain 
alternative proofs of some results established in [110]. Indeed, once the relevant properties have been obtained for auxiliary problems with $\mathbb{Q} \ll \mathbb{P}$, the proofs can be simplified. In particular, our approach enables establishing the relation between the primal and dual value functions (cf. (5.14) below) under slightly weaker assumptions than in [110]. However, for the existence of an optimizer, stronger assumptions are required. These assumptions, which are a consequence of the more general type of criteria we consider, are still economically feasible; see the discussion after Theorem 5.5 above.

The properties of the auxiliary problem that will be made use of are presented next.

Lemma 5.12. Let $U$ satisfy the Inada conditions ( $c f$. (5.2)) and assume $u_{Z}\left(x_{0}\right)<\infty$ for some $x_{0}>0$. Then, the function $u_{Z}(x)$ and $v_{Z}(y)$ defined, respectively, in (5.6) and (5.7) satisfy the following:

i) It holds that

$$
v_{Z}(y)=\sup _{x>0}\left(u_{Z}(x)-x y\right) \text { and } u_{Z}(y)=\inf _{y>0}\left(v_{Z}(x)+x y\right)
$$

and, furthermore,

$$
u_{Z}^{\prime}(0)=\infty \text { and } v_{Z}^{\prime}(\infty)=0
$$

ii) Under the additional assumption that $v_{Z}(y)<\infty, y>0$, it also holds that

$$
u_{Z}^{\prime}(\infty)=0 \text { and } v_{Z}^{\prime}(0)=-\infty .
$$

Moreover, the set $\left\{Z U^{+}(g): g \in \mathcal{C}(x)\right\}$ is $\mathbb{P}$-UI and the primal problem admits a solution.

For $\mathbb{Q} \sim \mathbb{P}$, the above result was established in $[79,80]$. For $\mathbb{Q} \ll \mathbb{P}$, note that while the objective in the auxiliary problem is defined with respect to the measure $\mathbb{Q}$, the set of strategies is still defined in the standard way with respect to the reference measure $\mathbb{P}$. This is the key reason for the above result to hold also for auxiliary problems defined with respect to $\mathbb{Q} \ll \mathbb{P}$ (cf. also Remark 5.15). Indeed, the proofs in $[79,80]$ make use of the assumption of NFLVR in order to treat the strategies (via the characterization in (5.37) below), not the objective function. In consequence, Lemma 5.12 follows by minor modifications the respective proofs in [79, 80]. For completeness, the details are presented in the Appendix. We note that the auxiliary problem also might be viewed as utility maximization under $\mathbb{P}$ with respect to the stochastic utility function $\tilde{U}(x)=Z_{T} U(x)$ and refer to, among others, [48] for alternative arguments. For an economic interpretation of the condition that $u_{Z}\left(x_{0}\right)<\infty$, see the discussion on p. 133 . 


\subsubsection{Proof of the existence of an optimal investment strategy}

The proof first establishes upper semicontinuity and quasi-concavity of the objective function. The existence of an optimizer then follows by use of a Komlos-type argument.

Proof of Theorem 5.5. For the case when $G(\cdot, t)$ is convex, we refer to Lemma 4.7 in [110]. Note that the optimization over $X \in \mathcal{X}(x)$ can be replaced by optimization over the set $\mathcal{C}(x):=\{g \in$ $\left.L_{+}^{0}\left(\mathcal{F}_{T}\right): g \leq X_{T}, X \in \mathcal{X}(x)\right\}$ of random variables. That is to say,

$$
u(x)=\sup _{g \in \mathcal{C}(x)} \inf _{\mathbb{Q} \in \mathcal{Q}} G\left(\mathbb{Q}, \mathrm{E}^{\mathbb{Q}}[U(g)]\right) .
$$

Due to assumption $(5.11), u_{\mathbb{Q}}(x)<\infty, \mathbb{Q} \in \mathbb{Q}$, (cf. Lemma 3.5 in [111]) and Lemma 5.12 applies. Hence, $\left\{U^{+}(f)\right\}, f \in \mathcal{C}(x)$ is $\mathbb{Q}$-UI, $\mathbb{Q} \in \mathcal{Q}$. Combined with an application of Fatou's Lemma to the negative part $U^{-}(f)$, this yields that $g \rightarrow \mathrm{E}^{\mathbb{Q}}[U(g)], g \in \mathcal{C}(x)$, is upper semicontinuous for $\mathbb{Q} \in \mathcal{Q}$. Since also $G(Z, \cdot)$ is upper semicontinuous and the pointwise infimum of u.s.c. functionals is again u.s.c., it follows that the mapping

$$
g \rightarrow V(g):=\inf _{\mathbb{Q} \in \mathcal{Q}} G\left(\mathbb{Q}, \mathrm{E}^{\mathbb{Q}}[U(g)]\right), \quad g \in \mathcal{C}(x)
$$

is upper semicontinuous as well. Moreover, as $g \rightarrow \mathrm{E}[Z U(f)]$ is concave, $G(Z, \cdot)$ is quasi-linear and the point-wise infimum of quasiconcave functionals is again quasiconcave, it follows that $g \rightarrow V(g)$ is quasiconcave. More precisely, the semicontinuity and quasi-concavity can be argued as follows. Since $t \rightarrow G(q, t)$ is non-decreasing and right-continuous, it holds according to Proposition B.2 in [35] that

$$
G(q, t) \geq m \text { if and only if } t \geq G^{(-1, l)}(q, m)
$$

where the left inverse is given by $G^{(-1, l)}(q, m)=\inf \{n \in \mathbb{R}: G(q, m) \geq n\}$. Consequently, the following holds:

$$
\{g \in \mathcal{C}(x): G(q,\langle U(g), q\rangle) \geq m\}=\left\{g \in \mathcal{C}(x):\langle q, U(g)\rangle \geq G^{(-1, l)}(q, m)\right\} .
$$

This level set is then closed and convex since $g \rightarrow\langle q, U(g)\rangle, g \in \mathcal{C}(x), q \in \mathcal{Q}$, is concave and upper semicontinuous according to the above. Then, in turn, it follows that the set

$$
\{g \in \mathcal{C}(x): V(g) \geq m\}=\cap_{q \in \mathcal{Q}}\{g \in \mathcal{C}(x): G(q,\langle U(g), q\rangle) \geq m\}
$$

is closed and convex and, thus, that $g \rightarrow V(g)$ is upper semicontinuous and quasiconcave.

The existence of an optimizer now follows by a standard Komlos-type argument. Indeed, let $\left(g_{n}\right)$ be a sequence in $\mathcal{C}(x)$ such that $V\left(g_{n}\right) \nearrow u(x)$. Since each $g_{n} \geq 0$, Komlos Lemma gives $\tilde{g}_{n} \in \operatorname{conv}\left(g_{n}, g_{n+1}, \ldots\right)$ converging $\mathbb{P}$-a.s. to some $g$. Since $\mathcal{C}(x)$ is convex, $\tilde{g}_{n} \in \mathcal{C}(x)$. 
Furthermore, according to Proposition 3.1 in [79], $\mathcal{C}(x)$ is closed under convergence in $L^{0}$ and, thus, $g \in \mathcal{C}(x)$. Next, due to the quasi-concavity of $g \rightarrow V(g)$ it holds that

$$
V\left(\tilde{g}_{n}\right)=V\left(\sum_{k \geq n} \lambda^{k} g_{k}\right) \geq \inf _{k \geq n} V\left(g_{k}\right)=V\left(g_{n}\right)
$$

Since $V\left(\tilde{g}_{n}\right) \leq u(x)$ and $V\left(g_{n}\right) \nearrow u(x)$ it, thus, follows that $\lim _{n \rightarrow \infty} V\left(\tilde{g}_{n}\right)=u(x)$; that is to say, $\tilde{g}_{n}$ is an optimizing sequence. Then, in turn, the upper semi-continuity of $g \rightarrow V(g)$ yields that

$$
V(g) \geq \limsup _{n \rightarrow \infty} V\left(\tilde{g}_{n}\right)=u(x)
$$

which concludes the proof.

\subsubsection{Proof of the duality results}

For the proof of Theorem 5.9, note that since $G(Z, \cdot)$ is non-decreasing, it immediately follows that

$$
u(x) \leq \inf _{\mathbb{Q} \in \mathcal{Q}} G\left(\mathbb{Q}, u_{\mathbb{Q}}(x)\right) \leq \inf _{\mathbb{Q} \in \mathcal{Q}} G\left(\mathbb{Q}, v_{\mathbb{Q}}(y)+x y\right)=v(y ; x), \quad \text { for all } y>0 .
$$

The proof verifies that the inequalities, in fact, are equalities by use of, respectively, Sion's minimax theorem and Lemma 5.12.

Proof of Theorem 5.9. Part i) For the case when $G(Z, \cdot)$ is concave and $U: \mathbb{R}_{+} \rightarrow \mathbb{R}_{+}$let, respectively, $\varepsilon>0$ and $\varepsilon=0$. According to (5.10), $u(x)<\infty, x>0$. Hence, for each $g \in \mathcal{C}(x)$,

$$
\inf _{Z \in \mathcal{Q}} G(Z, \mathrm{E}[Z U(\varepsilon+g)]) \leq \sup _{g \in \mathcal{C}(x)} \inf _{Z \in \mathcal{Q}} G(Z, \mathrm{E}[Z U(\varepsilon+g)]) \leq u(x+\varepsilon)<\infty .
$$

Consequently,

$$
\inf _{Z \in \mathcal{Q}} G(Z, \mathrm{E}[Z U(\varepsilon+g)])=\inf _{Z \in \tilde{\mathcal{Q}}} G(Z, \mathrm{E}[Z U(\varepsilon+g)]),
$$

where $\tilde{\mathcal{Q}}$ is the set of measures in $\mathcal{Q}$ for which $G(Z, \mathrm{E}[Z U(\varepsilon+g)]) \leq u(x+\varepsilon)+1$. Since $\mathrm{E}[Z U(\varepsilon+g)] \geq U(\varepsilon) \wedge 0$, for $Z \in \mathcal{Q}$, it holds for each $Z \in \tilde{\mathcal{Q}}$ that $G(Z, t) \leq c$ for $t:=U(\varepsilon) \wedge 0$ and $c:=u(x+\varepsilon)+1$. Hence, $\tilde{\mathcal{Q}}$ in $(5.24)$ might be replaced by $\mathcal{Q}_{t}(c)$. Since this holds for each $g \in \mathcal{C}(x)$, it thus follows that

$$
\sup _{g \in \mathcal{C}(x)} \inf _{Z \in \mathcal{Q}} G(Z, \mathrm{E}[Z U(\varepsilon+g)])=\sup _{g \in \mathcal{C}(x)} \inf _{Z \in \mathcal{Q}_{t}(c)} G(Z, \mathrm{E}[Z U(\varepsilon+g)])
$$

Next, as $U(\varepsilon+\cdot)$ is bounded from below and $\mathcal{Q}_{t}(c)$ is UI due to Assumption 5.8, an application of Fatou's Lemma yields $Z \rightarrow \mathrm{E}[Z U(\varepsilon+g)], g \in \mathcal{C}(x)$, lower semicontinuous with respect to a.s. convergence on $\mathcal{Q}_{t}(c)$. As $\mathcal{Q}_{t, T}$ is UI, that is equivalent to lower semicontinuity with respect 
to convergence in $L^{1}$. As the functional is convex (affine) that, in turn, implies weak lower semicontinuity. Since $G$ is jointly lower semicontinuous and quasiconvex it, thus, follows that

$$
Z \rightarrow G(Z, \mathrm{E}[Z U(\varepsilon+g)]), \quad g \in \mathcal{C}(x),
$$

is weakly lower semicontinuous and quasiconvex. Furthermore, as established above, it holds that

$$
g \rightarrow G(Z, \mathrm{E}[Z U(\varepsilon+g)]), \quad Z \in \mathcal{Q},
$$

is quasiconcave. Recall that $\mathcal{C}(x)$ is convex. Moreover,

$$
G(\lambda Z+(1-\lambda) \bar{Z}, t) \leq \max \{G(Z, t), G(\bar{Z}, t)\} \leq c,
$$

for $Z, \bar{Z} \in \mathcal{Q}_{t}(c)$. Hence, $\mathcal{Q}_{t}(c)$ is also convex. The latter set is also weakly compact due to Assumption 5.8. Given the properties of the mappings defined in (5.26) and (5.27), respectively, we might thus apply Sion's minimax theorem (cf. [114]). This yields,

$$
\sup _{g \in \mathcal{C}(x)} \inf _{Z \in \mathcal{Q}_{t}(c)} G(Z, \mathrm{E}[Z U(\varepsilon+g)])=\inf _{Z \in \mathcal{Q}_{t}(c)} \sup _{g \in \mathcal{C}(x)} G(Z, \mathrm{E}[Z U(\varepsilon+g)]) .
$$

Note that

$$
\inf _{Z \in \mathcal{Q}} \sup _{g \in \mathcal{C}(x)} G(Z, \mathrm{E}[Z U(\varepsilon+g)]) \leq \inf _{Z \in \mathcal{Q}_{t}(c)} \sup _{g \in \mathcal{C}(x)} G(Z, \mathrm{E}[Z U(\varepsilon+g)]) \leq u(x+\varepsilon),
$$

where the first inequality is trivial and the second follows from (5.28) combined with (5.25). Hence, by use of the same argument as above, the set $\mathcal{Q}_{t}(c)$ in the right hand side of (5.28) can be substituted for the set $\mathcal{Q}$. That is to say,

$$
\sup _{g \in \mathcal{C}(x)} \inf _{Z \in \mathcal{Q}} G(Z, \mathrm{E}[Z U(\varepsilon+g)])=\inf _{Z \in \mathcal{Q}} \sup _{g \in \mathcal{C}(x)} G(Z, \mathrm{E}[Z U(\varepsilon+g)]) .
$$

For the case when $U: \mathbb{R}_{+} \rightarrow \mathbb{R}_{+}$, this completes the proof of part i).

For the case when $G(Z, \cdot)$ is concave, a straight-forward argument yields $u(x)$ concave. According to (5.10) it is also finite. Consequently, it is continuous as a concave function is continuous on the interior of the set where it is finite (cf. Theorem 10.1 in [107]). On the other hand, since the expression on the left-hand-side in (5.29) clearly is smaller than $u(x+\varepsilon)$, it follows that

$$
\begin{aligned}
u(x+\varepsilon) & \geq \inf _{Z \in \mathcal{Q}} \sup _{g \in \mathcal{C}(x)} G(Z, \mathrm{E}[Z U(g)]) \\
& \geq \sup _{g \in \mathcal{C}(x)} \inf _{Z \in \mathcal{Q}} G(Z, \mathrm{E}[Z U(g)])=u(x) .
\end{aligned}
$$

Since $u(x)$ is (upper semi-) continuous, the result then follows by letting $\varepsilon \searrow 0$.

Next, according to Assumption 5.4, $\mathcal{Q}^{f} \neq \emptyset$. Since $G$ has an asymptotic maximum in the sense of Definition 5.1, this implies that the set $\mathcal{Q}$ on the right-hand side in (5.13) can be replaced 
by the set $\mathcal{Q}^{f}$. For $\mathbb{Q} \in \mathcal{Q}^{f}$, Lemma 5.12 applies. Hence, use of part i), the fact that $G(Z, \cdot)$ is non-decreasing and the duality relations between $u_{Z}(x)$ and $v_{Z}(y)$ given in Lemma 5.12, yields

$$
\begin{aligned}
u(x) & =\inf _{Z \in \mathcal{Q}^{f}} G\left(Z, u_{Z}(x)\right) \\
& =\inf _{Z \in \mathcal{Q}^{f}} G\left(Z, \inf _{y>0}\left(v_{Z}(y)+x y\right)\right)=\inf _{y>0} \inf _{Z \in \mathcal{Q}^{f}} G\left(Z, v_{Z}(y)+x y\right) .
\end{aligned}
$$

Given the definition of $v(y ; x)$, it thus only remains to show that

$$
\inf _{Z \in \mathcal{Q}^{f}} G\left(Z, v_{Z}(y)+x y\right)=\inf _{Z \in \mathcal{Q}} G\left(Z, v_{Z}(y)+x y\right)=: v(y ; x) .
$$

The inequality " $\geq$ " follows as $\mathcal{Q}^{f} \subseteq \mathcal{Q}$. Without loss of generality, assume $v(y ; x)<\infty$ and let $\left.\tilde{\mathcal{Q}}:=\left\{Z \in \mathcal{Q}: v_{Z}(y)<\infty\right)\right\}$. Clearly the set $\mathcal{Q}$ on the right-hand side of (5.31) can then be replaced by $\tilde{\mathcal{Q}}$. On the other hand, $v_{Z}(y)<\infty$ implies that $u_{Z}(x)<\infty$. Hence, $\tilde{\mathcal{Q}} \subseteq \mathcal{Q}^{f}$ which yields the inequality " $\leq$ ".

Part ii) Let

$$
H(Z, h):=G(Z, \mathrm{E}[Z V(h / Z)]+x y) .
$$

According to Lemma 3.7 in [111], $(Z, h) \rightarrow \mathrm{E}[Z V(h / Z)]$ is lower semicontinuous. Hence, since $G$ is jointly lower semicontinuous, it follows that so is $(Z, h) \rightarrow H(Z, h)$. Furthermore, since $(z, y) \rightarrow z V(y / z)$ is convex, $G(Z, \cdot)$ non-decreasing and $G$ jointly quasiconvex, it follows that $(Z, h) \rightarrow H(Z, h)$ is jointly quasiconvex. Indeed, let $Z_{t}=t Z_{0}+(1-t) Z_{1}$ and $h_{t}=t h_{0}+(1-t) h_{1}$.

Then,

$$
\begin{aligned}
H\left(Z_{t}, h_{t}\right) & =G\left(Z_{t}, \mathrm{E}\left[Z_{t} V\left(h_{t} / Z_{t}\right)\right]+x y\right) \\
& \leq G\left(Z_{t}, t \mathrm{E}\left[Z_{0} V\left(h_{0} / Z_{0}\right)\right]+(1-t) \mathrm{E}\left[Z_{1} V\left(h_{1} / Z_{1}\right)\right]+x y\right) \leq H\left(Z_{0}, h_{0}\right) \vee H\left(Z_{1}, h_{1}\right) .
\end{aligned}
$$

Let $\left(Z_{n}, h_{n}\right) \in \mathcal{Q} \times \mathcal{D}(y)$ be an optimizing sequence such that

$$
G\left(Z^{n}, \mathrm{E}\left[Z^{n} V\left(h^{n} / Z^{n}\right)\right]+x y\right) \underset{n \rightarrow \infty}{\searrow} v(y ; x)<\infty .
$$

Note that

$$
\mathrm{E}[Z V(h / Z)]+x y \geq U(x),
$$

for all $Z \in \mathcal{Q}$ and $h \in \mathcal{D}(y)$. Indeed, as $V$ is convex, use of Jensen's inequality yields

$$
\mathrm{E}[Z V(h / Z)]=\mathrm{E}\left[Z V\left(h / Z \mathbb{1}_{Z>0}\right)\right] \geq V\left(\mathrm{E}\left[Z h / Z \mathbb{1}_{Z>0}\right]\right)=V\left(\mathrm{E}\left[h \mathbb{1}_{Z>0}\right]\right) \geq V(y),
$$

as $V$ is decreasing and $h \in \mathcal{D}(y)$ which implies that $\mathrm{E}\left[h \mathbb{1}_{Z>0}\right] \leq \mathrm{E}[h] \leq y$. The fact that $G(Z, \cdot)$ is non-decreasing combined with (5.32) and (5.33), then yields

$$
\tilde{c}:=\limsup _{n \rightarrow \infty} G\left(Z^{n}, U(x)\right)<\infty
$$


and w.l.o.g., we can assume that $Z^{n} \in\{Z \in \mathcal{Q}: G(Z, U(x)) \leq \tilde{c}+1\}$. That is to say, that $Z^{n} \in \mathcal{Q}_{t}(c)$, for $t:=U(x)$ and $c:=\tilde{c}+1$.

Applying twice the Komlos Lemma, yields a sequence $\left(\tilde{Z}_{n}, \tilde{h}_{n}\right) \in \operatorname{conv}\left\{\left(Z_{n}, h_{n}\right),\left(Z_{n+1}, h_{n+1}\right), \ldots\right\}$ which converges $\mathbb{P}$-a.s. to some $\left(Z_{0}, h_{0}\right)$. Since $\mathcal{Q}_{t}(c)$ and $\mathcal{D}(y)$ both are convex, $\left(\tilde{Z}_{n}, \tilde{h}_{n}\right) \in$ $\mathcal{Q}_{t}(c) \times \mathcal{D}(y)$. Furthermore, since $\mathcal{Q}_{t}(c)$ is uniformly integrable (as it is weakly compact) and $\mathcal{D}(y)$ is closed in $L^{0}$ according to Proposition 3.1 in [79], it follows that $\left(Z_{0}, h_{0}\right) \in \mathcal{Q}_{t}(c) \times \mathcal{D}(y)$. Moreover, use of the quasiconvexity yields

$$
H\left(\tilde{Z}_{n}, \tilde{h}_{n}\right)=H\left(\sum_{k \geq n} \lambda^{k} Z_{k}, \sum_{k \geq n} \lambda^{k} h_{k}\right) \leq \max _{k \geq n} H\left(Z_{k}, h_{k}\right)=H\left(Z_{n}, h_{n}\right) \searrow v(y) .
$$

Hence, $\left(\tilde{Z}_{n}, \tilde{h}_{n}\right)$ is also an optimizing sequence. Consequently, by use of the lower semicontinuity, it follows that

$$
H\left(Z_{0}, h_{0}\right) \leq \liminf _{n \rightarrow \infty} H\left(\tilde{Z}_{n}, \tilde{h}_{n}\right)=v(y),
$$

which proves that the optimum is attained for $\left(Z_{0}, h_{0}\right)$.

Next, suppose $\left(\tilde{Z}_{1}, \tilde{h}_{1}\right)$ is another optimal pair. Let $h_{t}:=t \tilde{h}_{1}+(1-t) \tilde{h}_{0}$ and $Z_{t}:=t \tilde{Z}_{1}+(1-t) \tilde{Z}_{0}$, $t \in[0,1]$. As $(Z, h) \rightarrow \mathrm{E}[Z V(h / Z)]$ is convex and $G$ jointly quasiconvex, it thus follows that

$$
\begin{aligned}
G\left(Z_{t}, \mathrm{E}\left[Z_{t} V\right.\right. & \left.\left.\left(h_{t} / Z_{t}\right)\right]+x y\right) \\
& \leq G\left(Z_{t}, t \mathrm{E}\left[Z_{1} V\left(h_{1} / Z_{1}\right)\right]+(1-t) \mathrm{E}\left[Z_{0} V\left(h_{0} / Z_{0}\right)\right]+x y\right) \\
& \leq \max \left\{G\left(Z_{1}, \mathrm{E}\left[Z_{1} V\left(h_{1} / Z_{1}\right)\right]+x y\right), G\left(Z_{0}, \mathrm{E}\left[Z_{0} V\left(h_{0} / Z_{0}\right)\right]+x y\right)\right\}=v(y),
\end{aligned}
$$

due to the optimality of $\left(\tilde{Z}_{1}, \tilde{h}_{1}\right)$ and $\left(\tilde{Z}_{0}, \tilde{h}_{0}\right)$, respectively. Hence, also $\left(h_{t}, Z_{t}\right)$ is optimal. We proceed as in the proof of Lemma 4.3 in [110]. Note that for $t \in(0,1),\left\{Z_{t}>0\right\}=\left\{Z_{0}>\right.$ $0\} \cup\left\{Z_{1}>0\right\}$. Also note that according to $(25)$ in [110], the ration $h_{t} / Z_{t}$, does not depend on $t$. Hence, there exists a random variable $Y_{T} \geq 0$ and a sequence $\bar{Z}_{1}, \bar{Z}_{2}, \ldots$ such that:

(a) $\mathbb{P}\left[\bar{Z}_{n}\right]$ tends to the maximum $\mathbb{P}$-probability for the support of any optimal $Z$;

(b) $\left\{\bar{Z}_{1}>0\right\} \subseteq\left\{\bar{Z}_{2}>0\right\} \subseteq \ldots$;

(c) for each $n, \bar{h}_{n}:=Y_{T} \bar{Z}_{n} \in \mathcal{D}(y)$ and $\left(\bar{h}_{n}, \bar{Z}_{n}\right)$ is optimal.

By use of a Komlos-type argument, we may assume that $\bar{Z}_{n}$ converge $\mathbb{P}$-a.s. to some $\bar{Z} \in \mathcal{Q}$. Then $\bar{h}:=Y_{T} \bar{Z} \in \mathcal{D}(y)$ by Proposition 3.1 in [79] (cf. (5.37) below). As above, it follows that $(\bar{h}, \bar{Z})$ is optimal and, clearly, it is maximal.

Remark 5.13. Note that if it could be proven that $u(x)$ is upper semicontinuous, then Theorem 5.9 would hold for general utility functions from $\mathbb{R}_{+}$to $\mathbb{R}$. Indeed, the result would follow by 
simply exploiting upper semicontinuity of $u(x)$ in (5.30). Alternatively, it would be enough if one could verify that

$$
\lim _{\varepsilon \searrow 0} \sup _{g \in \mathcal{C}(x)} \inf _{Z \in \mathcal{Q}} G(Z, \mathrm{E}[Z U(\varepsilon+g)]) \leq \sup _{g \in \mathcal{C}(x)} \lim _{\varepsilon \searrow 0} \inf _{Z \in \mathcal{Q}} G(Z, \mathrm{E}[Z U(\varepsilon+g)]),
$$

as the right-hand-side of the above expression trivially (by use of upper semicontinuity and, thus, under assumptions similar to those in Theorem 5.5) is smaller than $u(x)$. A closer study of the properties of $u(x)$ is left for future study.

Next, we prove Theorem 5.10 which establishes the existence of a saddle point and the link between the primal and dual solutions.

Proof of Theorem 5.10. Part i) In order to verify the first statement, it remains to show that the infimum on the right hand side in (5.13) is attained. To this end, note that since $U \geq 0$, $G$ is jointly lower semicontinuous and quasiconvex and the operation of point-wise supremum preserves lower semicontinuity and quasiconvexity, it follows that

$$
Z \rightarrow \sup _{g \in \mathcal{C}(x)} G(Z, \mathrm{E}[Z U(g)])
$$

is lower semicontinuous and quasiconvex. Next, let $Z^{n} \in \mathcal{Q}$ be a sequence such that

$$
G\left(Z^{n}, u_{Z^{n}}(x)\right) \searrow u(x) .
$$

As $u_{Z^{n}}(x) \geq U(x)$, for all $n$ and $G(Z, \cdot)$ is increasing, it follows that

$$
\tilde{c}:=\limsup _{n \rightarrow \infty} G\left(Z^{n}, U(x)\right)<\infty .
$$

Hence, w.l.o.g., we may assume $Z^{n} \in \mathcal{Q}_{t}(c)$ with $t:=U(x)$ and $c=\tilde{c}+1$. Application of Komlos Lemma, yields a sequence $\tilde{Z}_{n} \in \operatorname{conv}\left\{Z_{n}, Z_{n+1}, \ldots\right\}$ which converges $\mathbb{P}$-a.s. to some $Z_{0}$. Since $\mathcal{Q}_{t}(c)$ is convex, $\tilde{Z}_{n} \in \mathcal{Q}_{t}(c), n=1,2, \ldots$. Furthermore, since $\mathcal{Q}_{t}(c)$ is uniformly integrable (as it is weakly compact), it follows that $Z_{0} \in \mathcal{Q}_{t}(c)$. Moreover, as in the proof of Theorem 5.9 part iii), the quasiconvexity implies that also $Z^{n}$ is an optimizing sequence. Use of the lower-semicontinuity of the mapping in (5.34), then yields that the infimum is attained for $Z_{0}$.

Part ii) Let $\tilde{g}$ and $\widetilde{Z}$ be a saddle-point for the primal problem. Let $y^{*}>0$ such that the infimum for the auxiliary conjugacy relations with respect to $\widetilde{Z}$ are attained for $y^{*}$. Then, it follows that

$$
u(x)=G\left(\widetilde{Z}, u_{\widetilde{Z}}(x)\right)=G\left(\widetilde{Z}, v_{\widetilde{Z}}\left(y^{*}\right)+x y^{*}\right) .
$$

Hence, the infimum in (5.14) is attained for $y^{*}$. Next, let $(\widehat{Z}, \hat{g})$ be any solution to the dual problem corresponding to $y^{*}$. Since $G(Z, \cdot)$ is non-decreasing, use of the assumptions and Lemma 5.12 yields

$$
u(x)=v\left(y^{*} ; x\right)=G\left(\hat{Z}, v_{\hat{Z}}\left(y^{*}\right)+x y^{*}\right) \geq G\left(\hat{Z}, u_{\hat{Z}}(x)\right) \geq u(x) .
$$


Hence, we have equality which, in turn, implies that

$$
u(x)=G\left(\hat{Z}, u_{\hat{Z}}(x)\right) \geq G(\hat{Z}, \mathrm{E}[\hat{Z} U(\hat{X})]) \geq \inf _{Z \in \mathcal{Q}} G(Z, \mathrm{E}[Z U(\hat{X})])=u(x) .
$$

Consequently, $(\widehat{\mathbb{Q}}, \widehat{X})$ is a saddle point for the primal problem.

Next, from the definition of $\mathcal{Y}(y)$, it follows that $\mathrm{E}\left[X_{T} Y_{T}\right] \leq x y$, for $X \in \mathcal{X}(x)$ and $Y \in \mathcal{Y}(y)$. Thus, it follows that

$$
\begin{aligned}
G(\hat{Z}, \mathrm{E}[\hat{Z} V(\hat{Y} / \hat{Z})+\hat{X} \hat{Y}])-G(\hat{Z}, \mathrm{E}[\hat{Z} U(\hat{X})]) & =G(\hat{Z}, \mathrm{E}[\hat{Z} V(\hat{Y} / \hat{Z})]+\mathrm{E}[\hat{X} \hat{Y}])-u(x) \\
& \leq G(\hat{Z}, \mathrm{E}[\hat{Z} V(\hat{Y} / \hat{Z})]+x y)-u(x) \\
& =v\left(y^{*} ; x\right)-u(x)=0 .
\end{aligned}
$$

Since $G(Z, \cdot)$ strictly increasing, this implies that

$$
\mathrm{E}^{\hat{\mathbb{Q}}}[V(\hat{Y} / \hat{Z})+\hat{X} \hat{Y} / \hat{Z}] \leq \mathrm{E}^{\hat{\mathbb{Q}}}[U(\hat{X})]
$$

On the other hand $V(\hat{Y} / \hat{Z})+\hat{X} \hat{Y} / \hat{Z} \geq U(\hat{X})$, $\hat{\mathbb{Q}}$-a.s. Consequently, $V(\hat{Y} / \hat{Z})+\hat{X} \hat{Y} / \hat{Z}=U(\hat{X})$ $\hat{\mathbb{Q}}$-a.s. and, thus, it follows that $\hat{X}=I(\hat{Y} / \hat{Z})$, $\hat{\mathbb{Q}}$-a.s.

\subsubsection{Proof of Lemma 5.12 and further remarks on the auxiliary prob- lem}

In this section we provide the proof of Lemma 5.12 and some further comments on the auxiliary problem. We stress that Lemma 5.12 follows by minor modifications in the respective proofs in $[79,80]$. Specifically, in the proofs of Lemmata 3.4 and 3.5 in [79] and Lemma 1 in [80]. For completeness, the details are presented next. For alternative arguments, see the further discussion in Section 5.3.1.

In preparation for the proof, note that the assumption $u_{\mathbb{Q}}(x)<\infty$ implies that the expectation operator is defined in the standard way (cf. page 130). It also immediately follows that

$$
u_{Z}(x)=\sup _{g \in \mathcal{C}(x)} \mathrm{E}[Z U(g)] \quad \text { and } \quad v_{Z}(y)=\inf _{h \in \mathcal{D}(y)} \mathrm{E}[Z V(h / Z)]
$$

where the set of random variables $\mathcal{C}(x)$ and $\mathcal{D}(y)$ are defined by $\mathcal{C}(x):=\left\{g \in L_{+}^{0}: g \leq\right.$ $X_{T}, \mathbb{P}$-a.s., $\left.X \in \mathcal{X}(x)\right\}$ and $\mathcal{D}(y)=\left\{h \in L_{+}^{0}: h \leq Z, \mathbb{P}\right.$-a.s., $\left.Z \in \mathcal{Y}(y)\right\}$. Moreover, according to Proposition 3.1 in [79], it holds that

$$
g \in \mathcal{C}(x) \text { if and only if } \mathrm{E}[g h] \leq x y, \text { for all } h \in \mathcal{D}(y) .
$$


Proof of Lemma 5.12. Consider the mapping $\mathcal{B}_{n} \times \mathcal{D}(y) \rightarrow \mathbb{R},(g, h) \rightarrow \mathrm{E}[Z U(g)-g h]$, where $\mathcal{B}_{n}:=\left\{g \in L_{+}^{0}: 0 \leq g \leq n\right\}$. The set $\mathcal{D}(y)$ is convex and since the unit ball in $L^{\infty}$ is weak*compact, so is $\mathcal{B}_{n}$. Furthermore, while the above mapping is concave in $g$, it is linear and continuous in $h$, this for the weak*-topology on $L^{0}$. Hence, the minimax theorem (cf. Theorem 2.7.1 in [6]) can be applied in order to obtain

$$
\sup _{g \in \mathcal{B}_{n}} \inf _{h \in \mathcal{D}(y)} \mathrm{E}[Z U(g)-g h]=\inf _{h \in \mathcal{D}(y)} \sup _{g \in \mathcal{B}_{n}} \mathrm{E}[Z U(g)-g h] .
$$

Next, (5.37) implies that

$$
\lim _{n \rightarrow \infty} \sup _{g \in \mathcal{B}_{n}} \inf _{h \in \mathcal{D}(y)} \mathrm{E}[Z U(g)-g h]=\sup _{x>0}\left(u_{Z}(x)-x y\right)
$$

Indeed, we first see that (5.37) implies that $\inf _{h \in \mathcal{D}(y)} \mathrm{E}[Z U(g)-g h] \geq \mathrm{E}[Z U(g)]-x y$. Taking the supremum over $g \in \mathcal{B}_{n}$ and $g \in \mathcal{C}(x) \cap \mathcal{B}_{n}$ on the left- and right-hand side, respectively and, then, in turn, letting $n \rightarrow \infty$ yields the inequality $\geq$ in (5.39). Next, we fix $n$ and let $g \in \mathcal{B}_{n}$ and $x^{*}:=\inf \{x>0: g \in \mathcal{C}(x)\}$. Without loss of generality, let $x^{*}>0$. Then it holds that $g \in \mathcal{C}\left(x^{*}+\varepsilon\right)$ but $g \notin \mathcal{C}\left(x^{*}-\varepsilon\right)$. Thus, using (5.37), it follows that

$$
\begin{aligned}
\inf _{h \in \mathcal{D}(y)} \mathrm{E}[Z U(g)-g h] & <\mathrm{E}[Z U(g)]-\left(x^{*}-\varepsilon\right) y \\
& \leq u_{Z}\left(x^{*}+\varepsilon\right)-\left(x^{*}+\varepsilon\right) y+2 \varepsilon y \leq 2 \varepsilon y+\sup _{x>0}\left(u_{Z}(x)-x y\right) .
\end{aligned}
$$

Letting $\varepsilon \searrow 0$, yields that for $g \in \mathcal{B}_{n}$ and $n \in \mathbb{N}$,

$$
\inf _{h \in \mathcal{D}(y)} \mathrm{E}[Z U(g)-g h] \leq \sup _{x>0}\left(u_{Z}(x)-x y\right) .
$$

This completes the proof of (5.39).

Next, let $V_{n}(y)=\sup _{0 \leq x \leq n}(U(x)-x y)$ and note that

$$
\begin{aligned}
\sup _{g \in \mathcal{B}_{n}} \mathrm{E}[Z U(g)-g h] & =\mathrm{E}\left[\sup _{0<x \leq n}\{Z U(x)-x h\}\right] \\
& =\mathrm{E}\left[\sup _{0<x \leq n}\left\{(Z U(x)-x h) \mathbb{1}_{Z>0}\right\}\right]=\mathrm{E}\left[Z J_{n}(h / Z)\right]
\end{aligned}
$$

where it was used that $\sup _{0<x<n}\{Z U(x)-x h\}=0$ on $\{Z=0\}$. Therefore, it holds that

$$
\inf _{h \in \mathcal{D}(y)} \sup _{g \in \mathcal{B}_{n}} \mathrm{E}[Z U(g)-g h]=\inf _{h \in \mathcal{D}(y)} \mathrm{E}\left[Z J_{n}(h / Z)\right]=: v_{Z}^{n}(y)
$$

Combining (5.38), (5.39) and (5.41), we easily see that in order to show the first conjugacy relation in i), it only remains to show that

$$
\lim _{n \rightarrow \infty} v_{Z}^{n}(y)=v(y), \quad y>0
$$

To this end, let $h_{n} \in \mathcal{D}(y)$ be a sequence such that

$$
\lim _{n \rightarrow \infty} v_{Z}^{n}(y)=\lim _{n \rightarrow \infty} \mathrm{E}\left[Z V^{n}\left(h_{n} / Z\right)\right]
$$


According to Komlos Lemma, there exist $\tilde{h}_{n} \in \operatorname{conv}\left(h_{n}, h_{n+1}, \ldots\right)$ converging $\mathbb{P}$-a.s. to some $h$ which belongs to $\mathcal{D}(y)$ as the latter set, according to Proposition 3.1 in [79], is closed under convergence in probability. Moreover, as $h \rightarrow z J(h / z)$ is convex (in [111], it is verified that also $(y, z) \rightarrow z V(y / z)$ is convex, this seems not needed here though) and $V^{n} \leq V^{m}, m \geq n$, it follows that

$$
\begin{aligned}
\inf _{m \geq n} \mathrm{E}\left[Z V^{m}\left(\tilde{h}_{m} / Z\right)\right] & \leq \mathrm{E}\left[Z V^{n}\left(\tilde{h}_{n} / Z\right)\right] \\
& \leq \sum_{m \geq n} \lambda^{m} \mathrm{E}\left[Z V^{n}\left(h_{m} / Z\right)\right] \leq \sup _{m \geq n} \mathrm{E}\left[Z V^{m}\left(h_{m} / Z\right)\right] .
\end{aligned}
$$

Hence, it holds that

$$
\lim _{n \rightarrow \infty} v_{Z}^{n}(y)=\limsup _{n \rightarrow \infty} \mathrm{E}\left[Z V^{n}\left(h_{n} / Z\right)\right] \geq \liminf _{n \rightarrow \infty} \mathrm{E}\left[Z V^{n}\left(\tilde{h}_{n} / Z\right)\right] .
$$

Consequently, if it can be shown that the set $\left\{Z V_{n}^{-}\left(\tilde{h}_{n} / Z\right): n \in \mathbb{N}\right\}$ is UI, then use of Fatou's Lemma yields

$$
\lim _{n \rightarrow \infty} v_{Z}^{n}(y) \geq \liminf _{n \rightarrow \infty} \mathrm{E}\left[Z V^{n}\left(\tilde{h}_{n} / Z\right)\right] \geq \mathrm{E}\left[Z V^{n}(h / Z)\right] \geq v_{Z}(y)
$$

where the last inequality follows as $h \in \mathcal{D}(y)$. Since, $v_{Z}^{n}(y) \leq v_{Z}(y)$, this concludes the proof of the first conjugacy relation.

It remains to establish the uniform integrability $\left\{Z V_{n}^{-}\left(\tilde{h}_{n} / Z\right): n \in \mathbb{N}\right\}$. To this end, note that for $I(y) \leq n$, it holds that $V_{n}(y)=V(y)$. As $V_{n}^{-}$is increasing in $y$ and decreasing in $n$, it thus follows that

$$
Z V_{n}^{-}\left(\tilde{h}_{n} / Z\right) \leq Z V^{-}\left(\tilde{h}_{n} / Z\right)+Z V_{1}^{-}\left(U^{\prime}(1)\right)
$$

Next, we note that $Z$ is integrable. According to Lemma 3.6 in [111], for a set $\mathcal{Q} \subset\{\mathbb{Q} \ll \mathbb{P}\}$ which is UI, it holds that the set $\left\{Z V^{-}(h / Z): h \in \mathcal{D}(y), Z \in \mathcal{Q}\right\}$ is UI. Hence, the uniform integrability of $\left\{Z V^{-}(h / Z): h \in \mathcal{D}(y)\right\}$ follows as a special case thereof. Hence, $\left\{Z V_{n}^{-}\left(\tilde{h}_{n} / Z\right): n \in \mathbb{N}\right\}$ is UI which completes the proof of the first conjugacy.

The reverse conjugacy follows directly from the first one. Indeed, due to assumption $u_{Z}\left(x_{0}\right)<\infty$ for some $x_{0}>0$. Hence, it is finite for all $x>0$ and, furthermore, it is concave. Consequently, the reverse conjugacy follows from Theorem 12.2 in [107].

Next, we turn to the proof of statement ii). Due to the conjugacy relations in i), the assumption that $v_{Z}(y)<\infty$ is equivalent to (cf. Note 1 in [80])

$$
\lim _{x \nearrow \infty} u_{z}(x) / x=0 .
$$

Hence, the $\mathbb{Q}$-uniform integrability of $\left(U^{+}\left(g_{n}\right)\right), n \geq 1$, can be established as in Lemma 1 in [80]. For completeness, we highlight the main steps. To this end, we assume contrary to the claim 
that the sequence is not $\mathbb{Q}$ - uniformly integrable. Then, (passing if necessary to a subsequence), one can find $\alpha>0$ and a disjoint sequence $\left(A^{n}\right)_{n \geq 1}$ of $(\Omega, \mathcal{F})$ such that, for $n \geq 1$,

$$
\mathrm{E}^{\mathbb{Q}}\left[U^{+}\left(g^{n}\right) \mathbb{1}_{A^{n}}\right] \geq \alpha .
$$

Define the sequence of random variables $\left(\tilde{g}^{n}\right)_{n \geq 1}$ by

$$
\tilde{g}^{n}=x_{0}+\sum_{k=1}^{n} g^{n} \mathbb{1}_{A^{n}}
$$

where $x_{0}:=\inf \{x>0: U(x) \geq 0\}$. For any $h \in \mathcal{D}(1)$, it then holds that under $\mathbb{P}$

$$
\mathrm{E}^{\mathbb{P}}\left[\tilde{g}^{n} h\right] \leq x_{0}+\sum_{k=1}^{n} \mathrm{E}^{\mathbb{P}}\left[\tilde{g}^{n} h\right] \leq x_{0}+n x .
$$

Hence, $\tilde{g}^{n} \in \mathcal{C}\left(x_{0}+n x\right)$. On the other hand, it holds that under $\mathbb{Q}$

$$
\mathrm{E}^{\mathbb{Q}}\left[U\left(\tilde{g}^{n}\right)\right] \geq \sum_{k=1}^{n} \mathrm{E}^{\mathbb{Q}}\left[U^{+}\left(\tilde{g}^{n}\right) \mathbb{1}_{A^{n}}\right] \geq \alpha n,
$$

and therefore,

$$
\limsup _{x \rightarrow \infty} \frac{u_{Z}(x)}{x} \geq \limsup _{x \rightarrow \infty} \frac{E^{\mathbb{Q}}\left[U\left(\tilde{g}^{n}\right)\right]}{x_{0}+n x} \geq \limsup _{x \rightarrow \infty} \frac{\alpha n}{x_{0}+n x}=\alpha>0 .
$$

This contradicts the assumptions (cf. (5.43)) which concludes the proof.

We conclude with some further remarks on the auxiliary value functions.

Remark 5.14. Let $\hat{\mathcal{Y}}(y)$ the set of all positive $\mathbb{Q}$-supermartingales such that $Y_{0}=y$ and $X Y$ is $a \mathbb{Q}$-supermartingale for all $X \in \mathcal{X}(1)$. Then, as shown in Lemma 4.2 in [110], it holds that

$$
v_{\mathbb{Q}}(y)=\inf _{Y \in \hat{\mathcal{Y}}(y)} \mathrm{E}^{\mathbb{Q}}\left[V\left(Y_{T}\right)\right] .
$$

Indeed, let $0 \leq s \leq t \leq T$. For $\hat{Y} \in \hat{\mathcal{Y}}(y)$ and $X \in \mathcal{X}(1)$,

$$
X_{s} \hat{Y}_{s} \geq \mathrm{E}^{\mathbb{Q}}\left[X_{t} \hat{Y}_{t} \mid \mathcal{F}_{s}\right]=\frac{1}{Z_{s}} \mathrm{E}\left[X_{t} \hat{Y}_{t} Z_{t} \mid \mathcal{F}_{s}\right], \quad \mathbb{P} \text {-a.s. on }\left\{Z_{s}>0\right\} .
$$

On $\left\{Z_{s}=0\right\}$, it holds that $Z_{t}=0 \mathbb{P}$-a.s. It follows that $X \hat{Y} Z$ is a $\mathbb{P}$-supermartingale and hence that $\hat{Y} Z \in \mathcal{Y}(y)$. Conversely, let $Y \in \mathcal{Y}(y)$. Then, $\mathbb{Q}$-a.s. for each $X \in \mathcal{X}(1)$,

$$
\mathrm{E}^{\mathbb{Q}}\left[X_{t} \frac{Y_{t}}{Z_{t}} \mid \mathcal{F}_{s}\right]=\frac{1}{Z_{s}} \mathrm{E}\left[Z_{t} X_{t} \frac{Y_{t}}{Z_{t}} \mathbb{1}_{Z_{t}>0} \mid \mathcal{F}_{s}\right] \leq \frac{1}{Z_{s}} \mathrm{E}\left[X_{t} Y_{t} \mathbb{1}_{Z_{s}>0} \mid \mathcal{F}_{s}\right] \leq \frac{X_{s} Y_{s}}{Z_{s}} \mathbb{1}_{Z_{s}>0} .
$$

Hence, $X Y / Z$ is a $\mathbb{Q}$-supermartingale, for all $x \in \mathcal{X}(x)$ and $Y / Z \mathbb{1}_{Z>0} \in \hat{\mathcal{Y}}(y)$. 
Remark 5.15. For $\mathbb{Q} \ll \mathbb{P}$, let $\mathcal{X}_{\mathbb{Q}}(x)$ the set of wealth-processes such that $X_{0} \leq x$ and $X_{t} \geq 0$, $\mathbb{Q}$-a.s. on $t \in[0, T]$ and $\mathcal{Y}_{\mathbb{Q}}(y)$ the set of all positive $\mathbb{Q}$-supermartingales such that $Y_{0}=y$ and $X Y$ is a $\mathbb{Q}$-supermartingale for all $X \in \mathcal{X}_{\mathbb{Q}}(1)$. Then, consider the following two problems

$$
\tilde{u}_{\mathbb{Q}}(x)=\sup _{X \in \mathcal{X}_{\mathbb{Q}}(x)} \mathbb{E}^{\mathbb{Q}}\left[U\left(X_{T}^{\pi}\right)\right] \quad \text { and } \quad \tilde{v}_{\mathbb{Q}}(y)=\inf _{Y \in \mathcal{Y}_{\mathbb{Q}}(y)} \mathrm{E}^{\mathbb{Q}}\left[V\left(Y_{T}\right)\right],
$$

where $\inf \emptyset:=\infty$. Note that $\tilde{u}(x)$ is the standard investment problem with respect to $\mathbb{Q}$ as it is normally defined. However, since it is not clear whether $S_{t}$ satisfies NFLVR with respect to $\mathbb{Q}$, it is (a priori) not clear whether $\tilde{u}$ and $\tilde{v}$ are each others conjugate. Also note that $\mathcal{X}(x) \subseteq \mathcal{X}_{\mathbb{Q}}(x)$, which, in turn, implies that $\mathcal{Y}_{\mathbb{Q}}(y) \subseteq \hat{\mathcal{Y}}(y)$. Hence,

$$
u(x) \leq \tilde{u}(x) \quad \text { and } \quad v_{\mathbb{Q}}(y) \leq \tilde{v}_{\mathbb{Q}}(y) .
$$

In particular, the condition that $\tilde{v}_{\mathbb{Q}}(y)<\infty, \mathbb{Q} \in \mathcal{Q}$, is therefore a sufficient condition for Theorem 5.5 to hold. For $\mathbb{Q} \sim \mathbb{P},(5.44)$ holds with equality. The question whether there are models $\mathbb{Q} \ll \mathbb{P}$, for which the inequality is strict is left for future study. We limit ourselves to note that given that the market is continuous, equality may in fact hold under rather weak conditions. Regardless of this, under the additional assumption (5.16), it holds for the variational case (i.e. when $G(\mathbb{Q}, t)=\gamma(\mathbb{Q})+t$ ) that

$$
v(y ; x)=\inf _{\mathbb{Q} \in \mathcal{Q}} G\left(\mathbb{Q}, \tilde{v}_{\mathbb{Q}}(y)+x y\right),
$$

and, in consequence, Theorem 5.9 holds also with $v_{\mathbb{Q}}(y)$ replaced by $\tilde{v}_{\mathbb{Q}}(y)$. Indeed, $v(y ; x)=$ $v(y)+x y$, where

$$
v(y)=\inf _{Z \in \mathcal{Q}^{e}}\left(v_{Z}(y)+\gamma(Z)\right)=\inf _{Z \in \mathcal{Q}^{e}}\left(\tilde{v}_{Z}(y)+\gamma(Z)\right) \geq \inf _{Z \in \mathcal{Q}}\left(\tilde{v}_{Z}(y)+\gamma(Z)\right) .
$$

Here the first equality follows from Lemma 4.4 in [110]. Since $v_{\mathbb{Q}}(y) \leq \tilde{v}_{\mathbb{Q}}(y), \mathbb{Q} \in \mathcal{Q}$, equality follows. 


\section{Chapter 6}

\section{Summary and Outlook}

The study of expected utility maximization in continuous-time stochastic market models dates back to the seminal contributions of Merton [91] and has since been central to the area of Mathematical Finance. The associated stochastic optimization problems have been extensively studied. The elegant theory of convex duality yield the existence and uniqueness of optimal strategies in great generality while explicit and closed form results have been obtained for specific settings through stochastic control methods. The problem formulation relies on two strong underlying assumptions: the ability to specify the underpinning market model and the knowledge of the investor's risk preferences. However, neither of these inputs is easily available, if at all. Resulting issues have attracted continuous attention and prompted very active and diverse lines of research. This thesis seeks to contribute towards this literature and questions related to both of the above issues have been studied.

In Chapter 2 it is shown that, in log-normal markets, important spatial and temporal properties of the optimal portfolios follow from monotonicity or concavity properties of the risk tolerance coefficient, regardless of the particular form of the utility function. Similar questions are also studied under structural assumptions on the marginal utilities and on their inverses. It is found that complete monotonicity of either of the two results in (complementary) general properties of the optimal portfolios. These properties are common for classes of HARA and CARA utilities for which both the marginal and its inverse are completely monotone.

The methods used in this chapter exploit the close relation between the investment problem in the log-normal market model and the one-dimensional heat equation. Hence, there is no immediate way to generalise these results to more general market models. Indeed, the temporal behaviour of the optimal policies have also been examined in more extended continuous time model settings in [27] (see also [84]). Therein, however, the generality of the model did not allow for specific results as the one herein. Although the precise results, thus, are expected to be 
quite specific for the log-normal model, we believe that suitable extensions of the relevant results for the heat equation, would enable an explicit study of similar questions also for more general stochastic factor models. Although challenging, we stress that the questions of how the shape of the utility function relates to the features of the investment strategy, are important for a further understanding of investors behaviour and risk preferences.

In Chapter 3 the notion of forward performance investment criteria is discussed. In particular, (time-monotone) forward investment-consumption criteria are studied in detail. For this notion, many interesting questions remain to be addressed. In particular, the question of how a restriction in the choice of the volatility process $a(x, t)$ can be related to investors' preferences. We also note that the developments for forward investment-consumption criteria opens up for a study of an associated equilibrium. Indeed, since the forward investment-consumption criteria constitute a new and alternative way of defining optimality of investments and consumption, it is natural to ask whether equilibrium could be defined with respect to such preferences. Following the classical literature, we say that the market is in equilibrium if the agents behave optimally and all markets clear. Hence, it seems natural to define an equilibrium corresponding to these criteria as a market which clears when all investors invest and consume optimally with respect to given forward investment-consumption criteria. It turns out that this is very challenging, mainly because it is conceptually difficult to define optimality for the investor who receives endowments and considers a forward investment-consumption criterion. Such questions are therefore left for future research.

Chapters 4 and 5 both focus on the study of feasible decision criteria in the presence of model uncertainty, also referred to as Knightian uncertainty. This topic originates from the seminal work of [54] who, by relaxing the independence axiom of von Neumann and Morgenstern, obtained a so-called numerical representation of the investor's preferences in terms of coherent monetary utility functionals. These axiomatic results were later generalized in [87] where the independence axiom was further relaxed leading to a (numerical) representation in terms of concave monetary utility functionals. Combining this with the representation of convex risk measures acting on (bounded) random variables motivated the evaluation of terminal payoffs according to

$$
\inf _{\mathbb{Q} \in \mathcal{Q}}\left(\mathrm{E}^{\mathbb{Q}}\left[U\left(X_{T}^{\pi}\right)\right]+\gamma(\mathbb{Q})\right) .
$$

The resulting problem of maximizing the criterion in (6.1) over a set of admissible trading strategies $\left(\pi_{t}\right)$ has been extensively studied within the mathematical finance literature. In particular, attention has been paid to the coherent (multiple priors) setting and the mathematically tractable case of entropic penalty functions introduced in [58]. For both, stochastic control methods have been successfully applied and solutions were obtained in terms of the associated BSDEs. This for the choice of specific utility functions as well as for stochastic differential utilities. 
In Chapter 4, it is investigated how to combine the above understanding of model uncertainty with the forward investment processes. A robust version of the forward performance criterion is introduced and, inspired by the works of [110] and [129], a suitable duality theory is developed. Interestingly, the time-consistency of optimal portfolios induces joint restrictions on the temporal evolution of the decision criteria and the associated family of penalty functions. A specific example is studied in detail and shown to result in optimal fractional Kelly strategies where the fraction to be invested depends on the investors trust in his market estimation. Several interesting aspects of this notion remain to be addressed. In particular, the question under which conditions a specific investment behaviour corresponds to some robust forward criterion. Indeed, as discussed in Chapter 4, the investment behaviour associated with a robust forward criterion is also optimal for a specific (standard) forward criterion. We think it is of interest to understand when the reverse implication holds and leave this for future research. As remarked above, we also expect that the results can be extended to set of absolutely continuous measures and leave for future research to thoroughly investigate this.

Chapter 5 focuses on ambiguity averse preferences in terms of quasiconcave utility functionals. This is motivated by the recent developments in [87] where the results in [25] were further generalised. By essentially removing the independence-axiom altogether, the authors obtained a (numerical) representation in terms of quasi-concave monetary utility functionals. Furthermore, advances in studies of risk measures (see [24] and [35]) yield that quasi-concave utility functionals admit the representation

$$
\inf _{\mathbb{Q} \in \mathcal{Q}} G\left(\mathbb{Q}, \mathrm{E}^{\mathbb{Q}}[X]\right), \quad X \in L^{\infty},
$$

for some function $G$ with given specific properties. This motivates the study of the risk- and ambiguity- averse investor who evaluates utility of terminal (not necessarily bounded) payoffs according to the quasi-concave functional in (6.2) and optimizes this quantity over a set of admissible strategies. In Chapter 5, relying on the classical results by [79, 80], existence of an optimal strategy is proven. Moreover, aspects of the duality theory established for the concave case in $[110]$ are extended and adjusted to this quasi-concave case.

It would be interesting to extend these results in order to obtain a fuller and richer understanding of investment choices under model uncertainty. In particular, to study related questions of timeconsistency of the preferences and the optimal policies. Indeed, time-consistency is essential for it is fundamentally linked to the definition and conceptual understanding of optimality. Moreover, it enables the use of stochastic control methods. Hence, it is a prerequisite for extending to the quasiconcave case the explicit results obtained in terms of BSDEs and PDEs for the variational preferences (cf. the references in Chapter 1). To this end, the time-consistency of quasi-convex risk measures needs, however, first be addressed. Indeed, while necessary and sufficient conditions 
for temporal consistency of convex risk-measures were established in [33] (see also [12] and [43]), such results are, yet, lacking for the quasi-convex case. In recent work, [49, 50] initiated such a programme via the study of conditional quasi-convex risk measures. Questions of temporal consistency and the relation to $g$-expectations have also been studied within the more general framework of non-linear expectations by [103]. It would be of interest to see to what extent these results could be used and how they can be extended in order to address the question of time-consistency of quasi-convex risk measures. In particular, it would be interesting to find an example of a quasiconcave utility functional defined in terms of a given BSDE and consider the associated investment problem.

We also note that in Chapter 5, the risk preferences of the investor were modelled via a standard continuous and concave utility function. While this assumption makes perfect sense for the static problem, the value function $u(x)$ will only satisfy weaker properties. This due to the weaker properties of the function $G$ for the quasiconcave, compared to the concave case. Hence, a study of time-consistent problems of this type also requires a study of the associated risk and ambiguity averse investment problem under weaker assumptions on $U(x)$. In particular, the related question of what properties the value-functions $u(x)$ and $v(y ; x)$ actually posses also needs to be addressed. In summary, while questions of time-consistency are important and of crucial interest, they impose challenging additional problems which are left for future study.

Finally, we note that a specific example of quasiconcave utility functionals considered in the literature are the so-called smooth criteria where an investor, rather than taking the infimum over possible measures, considers a distribution over them. Such criteria were axiomitized in Klibanoff et al. [77] under axioms stronger than those used in [25]. Hence, for bounded payoffs, these criteria constitute a particular case of the quasiconcave preferences. In particular, the specific form of the associated function $G$ is specified in [25]. This motivates the question of whether these results hold also for general terminal payoffs. We believe this to be the case but leave for future research to thoroughly investigate this. 


\section{Bibliography}

[1] B. Acciaio and V. Goldammer. Optimal portfolio selection via conditional convex risk measures on $L^{p}$. Decisions in Economics and Finance, 36(1):1-21, 2013.

[2] B. Acciaio and I. Penner. Dynamic risk measures. Advanced Mathematical Methods for Finance, ed. Di Nunno \& Øksendal, Springer, pages 1-34, 2011.

[3] E. Anderson, L. P. Hansen, and T. J. Sargent. A quartet of semigroups for model specification, robustness, prices of risk, and model detection. Journal of the European Economic Association, 1(1):68-123, 2003.

[4] M. Anthropelos. Forward exponential performance criteria: Pricing \& optimal risk sharing. ArXiv:1109.3908v3. 2013.

[5] K. J. Arrow. The theory of risk aversion. Aspects of the Theory of Risk Bearing, Yrjo Jahnssonin Saatio, Helsinki, 1965; reprinted in Essays in the Theory in Risk Bearing, North Holland, London, 1970.

[6] J. P. Aubin. Applied Functional Analysis. Wiley-Interscience, 2000.

[7] J. P. Aubin and I. Ekeland. Applied Nonlinear Analysis. New York, John Wiley \& Sons, 1984.

[8] F. P. Y. S. Berrier, L. C. G. Rogers, and M. R. Tehranchi. A characterization of forward utility functions: http://www.statslab.cam.ac.uk/ mike/papers/forward-utilityconsumption.pdf. 2009.

[9] F. P. Y. S. Berrier and M. R. Tehranchi. Forward utility of investment and consumption: http://www.statslab.cam.ac.uk/ mike/papers/forward-utilities.pdf. 2008.

[10] B. Bian, S. Miao, and H. Zheng. Smooth value functions for a class of nonsmooth utility maximization problems. SIAM Journal on Financial Mathematics, 2(1):727-747, 2011.

[11] J. Bion-Nadal. Conditional risk measure and robust representation of convex conditional risk measures. CMAP Preprint, 557, 2004. 
[12] J. Bion-Nadal. Time consistent dynamic risk processes. Stoch. Process. Appl., 119(2):633$654,2009$.

[13] T. Björk. Arbitrage theory in continuous time. Oxford University Press, 2009.

[14] T. Björk, A. Murgoci, and X. Y. Zhou. Mean-variance portfolio optimization with statedependent risk aversion. Mathematical Finance, 24(1):1-24, 2014.

[15] F. Black. Individual investment and consumption under uncertainty. Portfolio Insurance: A guide to dynamic hedging, D.L. Luskin (Ed.). New York, John Wiley and Sons, 1988, pages. 207-225; first version in 1968.

[16] F. Black. Investment and consumption through time. Financial Note No. 6B. Arthur D. Little, Inc., 1968.

[17] R. P. Boas. Entire functions. New York, Academic Press, 1954.

[18] G. Bordigoni, A. Matoussi, and M. Schweizer. A stochastic control approach to a robust utility maximization problem. Stochastic analysis and applications, 2:125-151, 2007.

[19] C. Borell. Monotonicity properties of optimal investment strategies for log-Brownian asset prices. Mathematical Finance, 17(1):143-153, 2007.

[20] C. Borell. Geometric properties of some familiar diffusions in $\mathbf{R}^{n}$. Ann. Probab., 21(1):482489, 1993.

[21] H. J. Brascamp and E. H. Lieb. Some inequalities for gaussian measures. Functional Integral and Its Applications, ed. by A. Arthurs., pages 1-14, 1975.

[22] P. L. Brocket and L. L. Golden. A class of utility functions containing all the common utility functions. Management Science, 33(8):955-964, 1987.

[23] C. Burgert and L. Rüschendorf. Optimal consumption strategies under model uncertainty. Statistics $\&$ Decisions, 23:1-14, 2005.

[24] S. Cerreia-Vioglio, F. Maccheroni, M. Marinacci, and L. Montrucchio. Complete monotone quasiconcave duality. Mathematics of Operations Research, 36:321-339, 2011.

[25] S. Cerreia-Vioglio, F. Maccheroni, M. Marinacci, and L. Montrucchio. Uncertainty averse preferences. Journal of Economic Theory, 146:1275-1330, 2011.

[26] Z. Chen and L. Epstein. Ambiguity, risk, and asset returns in continuous time. Econometrica, 70(4):1403-1443, 2002. 
[27] T. Choulli and M. Schweizer. The mathematical structure of horizon-dependence in optimal portfolio choice. Technical report, Working paper. http://www. math. ethz. ch/ mschweiz/ms_prep_eng. html, 2009.

[28] A. M. G. Cox, D. Hobson, and J. Obłój. Utility theory front to back - inferring utility from agents' choices. ArXiv:1101.3572v4. 2011.

[29] J. C. Cox and C. F. Huang. Optimal consumption and portfolio policies when assets follow a diffusion process. J. Economic Theory, 49(1):33-83, 1989.

[30] C. Czichowsky. Time-consistent mean-variance portfolio selection in discrete and continuous time. Finance Stoch., 17(2):227-271, 2013.

[31] F. Delbaen. Coherent risk measures on general probability spaces. In Advances in finance and stochastics, pages 1-37. Springer, 2002.

[32] F. Delbaen. The structure of $m$-stable sets and in particular of the set of risk neutral measures. In memorian Paul-Andreé Meyer: Séminaire de Probabilit'es XXXIX, Lecture notes in math., Springer Berlin, 1874, 2006.

[33] F. Delbaen, S. Peng, and E. R. Gianin. Representation of the penalty term of dynamic concave utilities. Finance Stochastics, 14:449-472, 2010.

[34] K. Detlefsen and G. Scandolo. Conditional and dynamic convex risk-measures. Finance and Stochastics, 9(4):539-561, 2005.

[35] S. Drapeau and M. Kupper. Risk preferences and their robust representation. Mathematics of Operations Research, 38:28-62, 2013.

[36] P. H. Dybvig and L. C. G. Rogers. Recovery of preferences from observed wealth in a single realization. The Review of Financial Studies, 10:151-174, 1997.

[37] N. El Karoui. Ecole d'Ete de Probabilites de Saint-Flour IX-1979. Springer Verlag, 1981.

[38] N. El Karoui and M. Mrad. An exact connection between two solvable SDEs and a nonlinear utility stochastic PDE. ArXiv:1004.5191v4. 2012.

[39] N. El Karoui and M. Mrad. Stochastic utilities with a given optimal portfolio: Approach by stochastic flows, ArXiv:1004.5192v2. 2013.

[40] W. Faidi, A. Matoussi, and M. Mnif. Maximization of recursive utilities: A dynamic maximum principle approach. SIAM J. Financial Mathematics, 2(1):1014-1041, 2011.

[41] D. Filipović, M. Kupper, and N. Vogelpoth. Approaches to conditional risk. Siam J. Financial Math., 3(1):402-432, 2012. 
[42] D. Filipović and G. Svindland. Convex risk measures on $L^{p}$ : http://www.math.lmu.de/ filipo/papers/crmlp.pdf. 2007.

[43] H. Föllmer and I. Penner. Convex risk measures and the dynamics of their penalty functions. Statistics \& Decisions, 24(1):61-96, 2006.

[44] H. Föllmer and A. Schied. Convex measures of risk and trading constraints. Finance and Stochastics, 6:429-447, 2002.

[45] H. Föllmer and A. Schied. Stochastic finance, an introduction in discrete time. Walter de Gruyter, 2nd edition, 2004.

[46] H. Föllmer, A. Schied, and S. Weber. Robust preferences and robust portfolio choice. Handbook of Numerical Analysis, 15:29-87, 2009.

[47] H. Föllmer and A. Gundel. Robust projections in the class of martingale measures. Illinois J. Math., 50(1-4):439-472 (electronic), 2006.

[48] H. Föllmer and P. Leukert. Efficient hedging: cost versus shortfall risk. Finance and Stochastics, 4(2):117-146, 2000.

[49] M. Fritelli and M. Maggis. Dual representation of quasi-convex conditional maps. Siam J. Financial Math., 2:357-382, 2011.

[50] M. Fritelli and M. Maggis. Complete duality for quasiconvex dynamic risk measures on modules of the $L^{p}$-type. ArXiv:1201.1788v2. 2012.

[51] M. Fritelli and E. Rosazza Gianin. Putting order in risk measures. J. Bank. Finance, 26:1473-1486, 2002.

[52] I. Fukuda, H. Ishii, and M. Tsutsumi. Uniqueness of solutions to the Cauchy problem for $u_{t}-u \Delta u+\gamma|\nabla u|^{2}=0$. Differential and Integral equations, 6(6):1231-1252, 1993.

[53] F. R. Gantmacher. Matrix Theory, volume 1. Chelsea Publishing Company, 1959.

[54] I. Gilboa and D. Schmeidler. Maxmin expected utility with non-unique prior. Journal of Mathematical Economics, 18:141-153, 1989.

[55] C. Gollier and R. J. Zeckhauser. Horizon length and portfolio risk. The Journal of Risk and Uncertainty, 24(3):195-212, 2002.

[56] C. Gollier and J. W. Pratt. Risk vulnerability and the tempering effect of background risk. Econometrica: Journal of the Econometric Society, 64(5):1109-1123, 1996.

[57] L. Guiso and M. Paiella. Risk aversion, wealth, and background risk. Journal of the European Economic Association, 6(6):1109-1150, 2008. 
[58] L. P. Hansen and T. J. Sargent. Robust control and model uncertainty. American Economic Review, 91:60-66, 2001.

[59] H. He and C. F. Huang. Consumption-portfolio policies: An inverse optimal problem. Journal of Economic Theory, 62(2):257-293, 1994.

[60] B. Heinz. Probability Theory. Walter de Gruyter, 1996.

[61] V. Henderson. Valuing the option to invest in an incomplete market. Mathematics and Financial Economics, 1(2):103-128, 2007.

[62] V. Henderson and D. Hobson. Horizon-unbiased utility functions. Stochastic Process. Appl., 117(11):1621-1641, 2007.

[63] D. A. Hennessy and H. E. Lapan. On the nature of certainty equivalent functionals. Journal of Mathematical Economics, 43(1):1-10, 2006.

[64] D. Hernández-Hernández and A. Schied. Robust utility maximization in a stochastic factor model. Statistics \& Decisions, 24(1):109-125, 2006.

[65] D. Hernández-Hernández and A. Schied. A control approach to robust utility maximization with logarithmic utility and time-consistent penalties. Stochastic Processes and their Applications, 117(8):980-1000, 2007.

[66] D. Hernández-Hernández and A. Schied. Robust maximization of consumption with logarithmic utility. American Control Conference, pages 1120-1123, 2007.

[67] Y. Hu, P. Imkeller, and M. Müller. Utility maximization in incomplete markets. The Annals of Applied Probability, 15(3):1691-1712, 2005.

[68] C. F. Huang and T. Zariphopoulou. Turnpike behavior of long-term investments. Finance and Stochastics, 3(1):1-20, 1999.

[69] M. Jeanblanc, A. Matoussi, and A. Ngoupeyou. Robust utility maximization in a discontinuous filtration. ArXiv:1201.2690v3. 2012.

[70] M. Kaina and L. Rüschendorf. On convex risk measures on $L^{p}$-spaces. Mathematical Methods of Operations Research, 69(3):475-495, 2009.

[71] I. Karatzas and C. Kardaras. The numéraire portfolio in semimartingale financial models. Finance ans Stochastics, 11:447-493, 2007.

[72] I. Karatzas, J. P. Lehoczky, and S. E. Shreve. Optimal portfolio and consumption decisions for a small investor on a finite horizon. SIAM J. Control Optim., 25(6):1557-1586, 1987. 
[73] I. Karatzas, J. P. Lehoczky, S. E. Shreve, and G. L. Xu. Martingale and duality methods for utility maximization in an incomplete market. SIAM J. Control Optim., 29(3):702-730, 1991.

[74] I. Karatzas and S. E. Shreve. Methods of Mathematical Finance. Springer, 1998.

[75] G. Keady. The persistence of logconcavity for positive solutions of the one dimensional heat equation. J. Austral. Math. Soc. Ser. A, 48:1-16, 1990.

[76] M. S. Kimball. Standard risk aversion. Econometrica, 61(3):589-611, 1993.

[77] P. Klibanoff, M. Marinacci, and S. Mukerji. A smooth model of decision making under amibiguity. Econometrica, 73(6):1849-1892, 2005.

[78] S. Klöppel and M. Schweizer. Dynamic utility indifference valuation via convex risk measures. Mathematical Finance, 17(4):599-627, 2007.

[79] D. Kramkov and W. Schachermayer. The asymptotic elasticity of utility functions and optimal investments in incomplete markets. Annals of Applied Probability, 9:904-950, 1999.

[80] D. Kramkov and W. Schachermayer. Necessary and sufficient conditions in the problem of optimal investment in incomplete markets. Annals of Applied Probability, 13:1504-1516, 2003.

[81] D. Kramkov and M. Sirbu. On the two-times differentiability of the value function in the problem of optimal investment in incomplete markets. Annals of Applied Probability, 16(3):1352-1384, 2006.

[82] R. J. A. Laeven and M. A. Stadje. Robust portfolio choice and indifference valuation: http://alexandria.tue.nl/repository/books/733411.pdf. 2012.

[83] F. Lajeri and L. T. Nielsen. Parametric characterizations of risk aversion and prudence. Economic Theory, 15(2):469-476, 2000.

[84] K. Larsen and H. Yu. Horizon dependence of utility optimizers in incomplete models. Finance and Stochastics, 16(4):779-801, 2012.

[85] A. Lazrak. Optimal martingale restrictions and horizon effect on portfolio selection, Prépublications de l'Equipe d'Analyse et Probabilités, No. 91, Université d'Évry: http://www. maths. univ-evry.fr/prepubli/index. html. 1998.

[86] A. Lazrak and M. C. Quenez. A generalized stochastic differential utility. Mathematics of Operations Research, 28(1):154-180, 2003. 
[87] F. Maccheroni, M. Marinacci, and A. Rustichini. Ambiguity aversion, robustness, and the variational representation of preferences. Econometrica, 74:1447-1498, 2006.

[88] M. A. Maggi, U. Magnani, and M. Menegatti. On the relationship between absolute prudence and absolute risk aversion. Decisions in Economics and Finance, 29(2):155-160, 2006.

[89] M. Mania and R. Tevzadze. Backward stochastic partial differential equations related to utility maximization and hedging. Journal of Mathematical Sciences, 153(3):291-380, 2008.

[90] E. Mastrogiacomo and E. Rosazza Gianin. Portfolio optimization with quasiconvex risk measures. http://ssrn.com/abstract=2282472. 2013.

[91] R. C. Merton. Lifetime portfolio selection under uncertainty: the continuous time case. The Review of Economics and Statistics, 51:247-257, 1969.

[92] R. C. Merton. Optimum consumption and portfolio rules in a continuous-time model. J. Economic Theory, 3(4):373-413, 1971.

[93] M. Müller. Market completion and Robust utility maximization. Dissertation, Humboldt Universität zu Berlin, 2005.

[94] M. Musiela and T. Zariphopoulou. Backward and forward utilities and the associated pricing systems: The case study of the binomial model. preprint, 2003.

[95] M. Musiela and T. Zariphopoulou. Portfolio choice under dynamic investment performance criteria. Quantitative Finance, 9(2):161-170, 2009.

[96] M. Musiela and T. Zariphopoulou. Portfolio choice under space-time monotone performance criteria. SIAM Journal on Financial Mathematics, 1(1):326-365, 2010.

[97] M. Musiela and T. Zariphopoulou. Stochastic partial differential equations and portfolio choice. Contemporary Quantitative Finance, C. Chiarella and A. Novikov (eds.), 2010.

[98] N. Nadtochiy and M. Tehranchi. Optimal investment for all time horizons and Martin boundary of space-time diffusions. preprint, 2011.

[99] S. Nadtochiy and T. Zariphopoulou. A class of homothetic forward investment performance processes with non-zero volatility. Inspired by Finance, Springer Verlag, in press, 2013.

[100] J. Neveu. Discrete Parameter Martingales. North-Holland Publishing Company, 1975.

[101] M. Nutz. Utility maximization under model uncertainty in discrete time, ArXiv:1307.3597v1. 2013. 
[102] K. Owari. Robust exponential hedging in a brownian setting. Mimeo, Hitotsubashi University, 2009.

[103] S. Peng. Dynamically consistent nonlinear evaluations and expectations. ArXiv:math/0501415v1. 2005.

[104] J. W. Pratt and R. J. Zeckhauser. Proper risk aversion. Econometrica: Journal of the Econometric Society, 55(1):143-154, 1987.

[105] M. H. Protter and H. F. Weinberger. Maximum principles in differential equations. Springer Verlag, New York, 1984.

[106] M. C. Quenez. Optimal portfolio in a multiple-priors model. In Seminar on Stochastic Analysis, Random Fields and Applications IV, pages 291-321. Springer, 2004.

[107] R. T. Rockafellar. Convex Analysis. Princeton University Press, 1970.

[108] P. A. Samuelson. The judgment of economic science on rational portfolio management: indexing, timing, and long-horizon effects. The Journal of Portfolio Management, 16(1):412,1989 .

[109] W. Schachermayer. Optimal investment in incomplete markets when wealth may become negative. Annals of Applied Probability, 11(3):694-734, 2001.

[110] A. Schied. Optimal investments for risk- and ambiguity-averse preferences: a duality approach. Finance and Stochastics, 11:107-129, 2007.

[111] A. Schied and C. T. Wu. Duality theory for optimal investments under model uncertainty. Statistics $\& 3$ Decisions, 23(3):199-217, 2005.

[112] I. J. Schoenberg. On Polya frequency functions I: The totally positive functions and their Laplace transforms. Journal d'Analyse Mathématique, 1951.

[113] M. Simonnet. Measures and Probabilities. Springer, 1996.

[114] M. Sion. On general minimax theorems. Pacific Journal of Mathematics, 8:171-176, 1958.

[115] C. Skiadas. Robust control and recursive utility. Finance and Stochastics, 7(4):475-489, 2003.

[116] T. Strzalecki. Axiomatic foundations of multiplier preferences. Econometrica, 79:47-73, 2011.

[117] P. Vitória and T. Zariphopoulou. Infinitesimal mean-variance analysis: convergence and time-consistency. preprint, 2013. 
[118] J. Von Neumann and O. Morgenstern. Theory of games and economic behavior (commemorative edition). Princeton university press, 2007.

[119] N. Westray and H. Zheng. Minimal sufficient conditions for a primal optimizer in nonsmooth utility maximization. Finance and Stochastics, 15(3):501-512, 2011.

[120] D. V. Widder. The Laplace Transform. Princeton University Press, 1946.

[121] D. V. Widder. Necessary and sufficient conditions for the representation of a function by a Weierstrass transform. Transactions of the American Mathematical Society, 71(3):430-439, 1951.

[122] D. V. Widder. The convolution transform. Bulletin of the American Mathematical Society, 60(5):444-456, 1954.

[123] D. V. Widder. The Heat Equation. Academic Press, 1975.

[124] W. Wittmüß. Robust optimization of consumption with random endowment. Stochastics An International Journal of Probability and Stochastic Processes, 80(5):459-475, 2008.

[125] J. Xia. Risk aversion and portfolio selection in a contiuous-time model. SIAM J. Control Optim., 49(5):1916-1937, 2011.

[126] T. Zariphopoulou. A solution approach to valuation with unhedgeable risk. Finance and Stochastics, 5(1):61-82, 2001.

[127] T. Zariphopoulou. Optimal asset allocation in a stochastic factor model - an overview and open problems. Advanced financial modelling, Radon series in Computational and Applied Mathematics, 8:427-453, 2009.

[128] T. Zariphopoulou and G. Zitković. Maturity-independent risk measures. SIAM Journal on Financial Mathematics, 1(1):266-288, 2010.

[129] G. Zitković. A dual characterization of self-generation and exponential forward performances. Annals of Applied Probability, 19:2176-2210, 2009. 